\title{
Optimising outcomes after liver transplantation
}

Citation for published version (APA):

Reiling, J. (2017). Optimising outcomes after liver transplantation: Pathophysiology of biliary injury and the application of machine perfusion. [Doctoral Thesis, Maastricht University]. Proefschriftmaken.nl || Uitgeverij BOXPress. https://doi.org/10.26481/dis.20170426jr

Document status and date:

Published: 01/01/2017

DOI:

10.26481/dis.20170426jr

Document Version:

Publisher's PDF, also known as Version of record

\section{Please check the document version of this publication:}

- A submitted manuscript is the version of the article upon submission and before peer-review. There can be important differences between the submitted version and the official published version of record.

People interested in the research are advised to contact the author for the final version of the publication, or visit the DOI to the publisher's website.

- The final author version and the galley proof are versions of the publication after peer review.

- The final published version features the final layout of the paper including the volume, issue and page numbers.

Link to publication

\footnotetext{
General rights rights.

- You may freely distribute the URL identifying the publication in the public portal. please follow below link for the End User Agreement:

www.umlib.nl/taverne-license

Take down policy

If you believe that this document breaches copyright please contact us at:

repository@maastrichtuniversity.nl

providing details and we will investigate your claim.
}

Copyright and moral rights for the publications made accessible in the public portal are retained by the authors and/or other copyright owners and it is a condition of accessing publications that users recognise and abide by the legal requirements associated with these

- Users may download and print one copy of any publication from the public portal for the purpose of private study or research.

- You may not further distribute the material or use it for any profit-making activity or commercial gain

If the publication is distributed under the terms of Article $25 \mathrm{fa}$ of the Dutch Copyright Act, indicated by the "Taverne" license above, 
Optimising outcomes after liver transplantation

Pathophysiology of biliary injury and the application of machine perfusion 
(C) Janske Reiling, Maastricht 2017

All rights reserved. No part of this publication may be reproduced or transmitted, in any form or by any means, without the permission from the copyright owner.

Layout: Tiny Wouters

Cover photo: Thijs Reiling

Cover design: ProefschriftMaken || www.proefschriftmaken.n|

Print: $\quad$ ProefschriftMaken II www.proefschriftmaken.nl

ISBN: 978-94-6295-556-1

Financial support: The work presented in this thesis was funded by the Gallipoli Medical Research Foundation and the Liver Transplant Bequest from the PA Research Foundation, Princess Alexandra Hospital, Brisbane, Australia. 


\title{
Optimising outcomes after liver transplantation
}

\author{
Pathophysiology of biliary injury and the application of \\ machine perfusion
}

\section{Dissertation}

To obtain the degree of Doctor of Philosophy from

The University of Queensland and the degree of Doctor at Maastricht University, on the authority of Rector Magnificus Prof. dr. Rianne M. Letschert, according to the decision of the Board of Deans

to be defended in public on

Wednesday, April 26, 2017 at 12.00 hours

by

Janske Reiling, MD

Born on October 31, 1987

in Maarheeze (The Netherlands) 


\section{Supervisor}

Prof. dr. J. Fawcett, University of Queensland

Prof. dr. C.H.C. Dejong

\section{Co-supervisor}

Prof. dr. D.H.G. Crawford, University of Queensland

Dr. K.R. Bridle, University of Queensland

\section{Doctoral Thesis Committee}

Prof. dr. A. A.M. Masclee (Chairman)

Prof. dr. J.N.M. IJzermans (Erasmus MC, Rotterdam)

Prof. dr. P.L.M. Jansen

Prof. dr. H. Pleass (Westmead hospital, Sydney, Australia)

Prof. dr. G.W.H. Schurink

The research project leading to this thesis was conducted as a Doctor of Philosophy Program in collaboration between the University of Queensland and Maastricht University. 


\section{Contents}

Chapter 1 General Introduction and Aims 7

Chapter 2 Increased liver non-use: The implications of the shift towards 19 Donation after Circulatory Death in Australia Submitted

Chapter 3 Low dose lipopolysaccharide causes biliary injury by blood biliary barrier impairment in a rat hepatic ischaemia-reperfusion model Liver Transplantation, 2016 Nov 23. doi: 10.1002/It.24681

Chapter 4 The role of macrophages in the development of biliary injury in a lipopolysaccharide enhanced hepatic ischaemia-reperfusion model Submitted

Chapter 5 Comparable levels of inflammatory mediators in portal venous blood collected from organ donors donating after circulatory death and those donating after brain death Submitted

Chapter 6 Machine perfusion of the human liver: A systematic review Submitted

Chapter 7 Urea production during normothermic machine perfusion- Price of success?

Liver Transplantation, 2015;21(5):700-3

Chapter 8 Does normothermic machine perfusion preserve bile ducts as well as it preserves hepatocytes? A study of human donor livers Submitted

Chapter 9 General discussion

Summary

Samenvatting

Valorisation

Acknowledgements

List of publications 



\section{Chapter 1}

General introduction and Aims 



\section{History of Liver transplantation}

The history of liver transplantation dates back to the mid-20 ${ }^{\text {th }}$ century. The first orthotopic liver transplant in a canine model was performed by Vittorio Staudacher in Milan in $1952 .{ }^{1}$ Unfortunately at that time, his breakthrough was not recognized by the greater scientific community and not Staudacher but Stuart Welch and Jack Cannon were credited with performing the first heterotopic and orthotopic liver transplantation in large animal models in 1955 and 1956 respectively. ${ }^{2,3}$ Based on the extensive experience in dogs, the first liver transplant in humans was performed by Thomas Starzl in $1963 .{ }^{4}$ Unfortunately, none of his four patients described in his initial report survived past the 1-month mark and it would take him another 4 years to perfect the technique. Between July 23, 1967 and March 17, 1968 Starzl successfully transplanted seven consecutive patients with biliary atresia or hepatoblastoma, all of which were children. ${ }^{5}$ At the time of publication, the longest surviving recipient was still alive 9 months post transplantation. Key to his success were measures implemented to reduce the degree of ischemic insult to the liver. Cardiac massage and ventilatory support were initiated post mortem, the liver was rapidly cooled in situ using balanced electrolyte solution, and an ex situ oxygenated hypothermic organ perfusion device was used to bridge the period between donation and implantation.

Soon after the successful report by Starzl, Calne and Moore reported their first experience with human liver transplantation in Europe in a joint venture between the Addenbrooke's hospital in Cambridge and King's College Hospital, London. ${ }^{6}$ In their initial series of five patients published in 1968, they presented the first case of heterotopic liver transplantation in humans (the liver was placed in the splenic bed). Furthermore, they performed a transplant technique currently still used in clinical practice where the recipient inferior vena cava (IVC) was preserved and the donor liver was 'piggybacked' on the recipient IVC.

In the 10 years that followed, patient survival substantially improved. The acceptance of brain death criteria in 1968 and the introduction of cyclosporine by Calne in $1979^{7}$ lead to higher quality organs and substantially improved graft survival. ${ }^{8}$

In 1983, the National Institutes of Health in the USA hosted a consensus conference on liver transplantation. Up until that point, 540 human liver transplants had been performed in four medical centres in the US and Western Europe. ${ }^{9}$ During the meeting, the outcomes of these procedures were compared with patients who did not undergo liver transplantation. The transplant centre in Pittsburgh, led by Starzl, had performed by far the most liver transplants up until that point. ${ }^{8}$ Since the start of the program in 1963, 296 patients had undergone liver transplantation. The introduction of cyclosporine had a major impact on patient survival with 1-year survival rates of 
32.9\% before 1980 and $70 \%$ between 1980 and 1982. Calne and colleagues in Cambridge and King's College performed 138 liver transplants between 1968 and 1983, predominantly for hepatic malignancy. Their survival rates were inferior to those reported by Starzl with reported rates of $30-40 \%$ on average. ${ }^{10}$ Pichlmayr and colleagues in Hannover, Germany, reported outcomes of 90 orthotopic transplants performed at their centre since $1979 .{ }^{11}$ Only $20 \%$ of patients that underwent transplantation for hepatic malignancy survived longer than 1 year. Cirrhotic patients that were transplanted in an elective setting however had the best chances of survival with $80 \%$ survival past the 1-year mark. The last series of 26 consecutive liver transplantations considered during the consensus conference were performed in Groningen, the Netherlands by Slooff and colleagues. ${ }^{12}$ Although this transplant program was by far the smallest, careful patient selection and the technique of biliary anastomosis had resulted in 1 and 2 year survival rates of $60 \%$. Ultimately, the panel decided that survival advantages of patients undergoing this procedure had clearly been demonstrated and liver transplantation should no longer be considered experimental. Following on from this meeting, the pioneering centres started training other transplant teams and new liver transplant units were established around the globe.

Despite fierce opposition from both the medical and the political world, Professor Russell Strong and his team at the Princess Alexandra hospital carried out the first adult orthotopic liver transplant in Australia on the $29^{\text {th }}$ of January $1985 .{ }^{13}$ This was successful and he went on to perform the first paediatric transplant 6 weeks later. During his short visit to Pittsburgh prior to establishing a transplant centre in Brisbane, Strong realised that a shortage of paediatric donors would remain a limiting factor in paediatric transplantation. This led him initially to develop a strategy by which an adult liver could be "cut down" to a size suitable for a child - this technique is currently still referred to as the Brisbane technique. ${ }^{14}$ Subsequently, he was the first in the world to successfully develop a technique where parents could be a living donor for their child. In July 1989, a segment 2/3 graft from a mother was successfully transplanted into her 17 -month old son who suffered from biliary atresia. ${ }^{15}$

\section{Liver transplantation in the 21th century}

In the current era of liver transplantation, the shortage of suitable donors is a pressing problem. Annually, over 500 patients with end stage liver disease are placed on the transplant waiting list throughout Australia. However only $50 \%$ will receive their lifesaving liver transplantation within a year of listing. ${ }^{16}$ As many as $10 \%$ of patients on the waiting list died or their disease progresses to a point where liver transplantation 
is not considered a suitable treatment option anymore. To expand the number of available organs, extended criteria donors are being considered for transplantation. ${ }^{17}$ An important group of such organs are those donated after circulatory death (DCD). DCD organs are retrieved from donors allowed to progress to circulatory death after withdrawal of ventilatory and circulatory support and are therefore exposed to a period of warm ischaemia prior to cold perfusion. This in turn makes DCD livers more susceptible to cold preservation injury and recipients of these grafts frequently develop complications. ${ }^{18}$ Although short-term graft survival rates have been reported to be similar to that of livers from brain dead donors (DBD), long-term graft survival is significantly reduced. ${ }^{19,20}$ The analysis of a DCD liver recipient cohort from the Netherlands and Belgium, followed for up to 10 years, showed that 5-year graft survival of DCD livers was $54 \%$ compared to $66 \%$ of DBD controls $(p=0.02)$. Patient survival was similar between the two categories. ${ }^{20}$

\section{Part I: Biliary stricture formation following DCD liver transplantation}

One of the most important complications following transplantation of a DCD liver grafts is the development of biliary strictures, more specifically ischaemic type biliary strictures (ITBS) which are also referred to as non-anastomotic strictures (NAS). ITBS develops in between 10 and 33\% of DCD recipients and the severity of disease is much greater compared to DBD recipients that develop ITBS. ${ }^{18,19,21}$ These strictures are mainly localised around or below the bifurcation of the common bile duct and occur within the first year of transplantation in over half the cases. ${ }^{22}$ Treatment options for ITBS are limited and consist mainly of endoscopic dilatation and stent placement. ${ }^{23}$ Ultimately, re-transplantation is required in a large proportion of ITBS sufferers. ${ }^{21}$ The pathophysiology of ITBS still remains largely unknown. Currently known risk factors mainly originate from case cohort studies however little is known about the true mechanistic processes involved. ${ }^{24}$ However studies assessing the pathophysiology of other diseases such as primary sclerosing cholangitis could provide helpful clues as to which processes could be responsible for the development of biliary stricture formation following transplantation of a DCD liver graft.

$M d r 2^{--}$mice lack the canalicular phospholipid flippase transporter and as a result, no phospholipid is being excreted in bile. These animals develop severe biliary injury ultimately resulting in biliary stricture formation closely resembling the appearance of primary sclerosing cholangitis. ${ }^{25}$ Fickert at al. studied the sequences of events leading to the development of these strictures and found that the disruption of the bloodbiliary-barrier (BBB) played a critical role in disease progression. ${ }^{26}$ The BBB consists of 
tight junctions between adjacent hepatocytes or cholangiocytes, and serves as a natural barrier separating blood from bile. ${ }^{27}$ In the $m d r 2^{-1-}$ mice, disruption of this barrier lead to leakage of toxic bile salts into the portal tract area. ${ }^{26}$ This in turn caused portal tract inflammation which ultimately induced periductal fibrosis and epithelial cell death. Primary sclerosing cholangitis (PSC) shows significant similarities with ITBS with regards to radiographic appearance. In fact, PSC recurrence after transplant is indistinguishable from the development of ITBS based on imaging. This lead us to hypothesise that dysfunction of the BBB could potentially play a role in ITBS formation, especially since increased bile toxicity early after transplantation has previously been associated with the development of ITBS. ${ }^{28}$

Most insights into the modulation of this BBB result from cell culture experiments. Oxidative stress a well as pro-inflammatory cytokines like Tnf- $\alpha$ and Ifn- $\gamma$ were found to increase permeability of the BBB. ${ }^{29,30}$ Furthermore, lipopolysaccharides (LPS), the outer capsule of gram negative bacteria, induced tight junction disruption and increased para-cellular permeability in a time and dose dependent fashion. ${ }^{31}$ This effect was mediated by Toll-Like Receptor 4 (TLR-4) and LPS binding protein signalling pathways.

Currently, little is known about the integrity of the blood-biliary barrier following liver transplantation. A study examining common bile duct samples from DBD livers at time of retrieval, end of cold storage and just before the biliary anastomosis was made showed a marked destruction of tight junction architecture in patients with histologically severe bile duct damage. ${ }^{32}$ These patients were more likely to go on and develop biliary complications and graft loss. Although formal evidence is lacking, gut ischaemia during the DCD organ retrieval process is likely to occur which could ultimately result in the appearance of endotoxins in portal blood being flushed through the liver. We therefore hypothesised that exposure to endotoxins, in addition to ischaemia, would affect the BBB and, in turn, induce biliary stricture formation.

\section{Part II: Application of machine perfusion}

The application of machine perfusion to preserve organs ex vivo was first described by Alexis Carrel and Charles Lindberg in $1935 .^{33}$ They built a normothermic perfusion apparatus, which allowed them to study whole organs such as heart, kidneys and ovaries for several days ex vivo. Several decades later, Starzl used a normothermic perfusion machine when he performed the first successful liver transplant. ${ }^{5}$ In the 1980's and 90's, the shift towards the use of brain death donors as well as the development of University of Wisconsin solution meant that organ quality significantly 
increased and the complex and comprehensive technique of machine perfusion was largely abandoned. As currently more DCD livers are considered for transplantation, alternative organ preservation methods such as machine perfusion are explored yet again.

Compared to static cold storage, machine perfusion holds the potential to better preserve organ function, it allows for prolonged preservation thereby facilitating long distance transport and it allows for viability assessment of the graft prior to implantation. Currently, normothermic machine perfusion (NMP) is the only technique that allows for viability assessment as the metabolic functions of liver grafts are preserved at $37^{\circ} \mathrm{C}$. Successful use of NMP has been described in different animal models. ${ }^{34-37}$ Furthermore, a pilot study using declined human donor livers has shown that NMP is technically feasible and allows assessment of graft viability. ${ }^{38}$ Besides the ability to assess metabolic processes in the liver, NMP offers a unique opportunity to study bile duct biology in a near physiological setting. 


\section{Aims of the thesis}

The overarching aim of this thesis was to characterise the sequence of events associated with the development of ITBS following transplantation of DCD livers. To achieve this, we used both a partial hepatic ischaemia reperfusion model in rats as well as ex vivo normothermic machine perfusion of human donor livers. We hypothesised that increased permeability of the BBB, in combination with bile toxicity, was associated with the development of ITBS following transplantation of DCD liver grafts.

In Chapter 2 we determined the impact of DCD donation on the number of livers available for transplantation in Australia over the last decade. We furthermore assessed the risk factors associated with liver non-use.

\section{Aims Part I: the development of biliary injury}

The aim of part I of this thesis was to develop an animal model for biliary injury. As liver transplantation in rodent models is rather difficult, and requires extensive microsurgical training, we choose a much simpler and more reproducible model of partial hepatic ischaemia-reperfusion. We furthermore aimed to test the attributive role of cofactors such as endotoxins in the development of biliary injury in this model. Lastly we aimed to develop a method to assess the permeability of the BBB in vivo.

In Chapter $\mathbf{3}$ we tested the role of endotoxins, in the form of LPS, in the development of biliary injury in a partial liver ischaemia-reperfusion model in rats. In this chapter, we further describe the use of horseradish peroxidase to assess the permeability of the BBB in vivo.

Chapter $\mathbf{4}$ explores the role of Kupffer cells in the development of LPS-induced biliary injury. Clodronate liposomes were used to deplete Kupffer cells from the liver and animals were subsequently exposed to LPS or a combination of warm hepatic ischaemia and LPS.

As it remained unclear if endotoxaemia occurred in DCD donors, we assessed the presence of inflammatory mediators in portal venous blood of both DBD and DCD donors during organ procurement in Chapter 5. We hypothesised that unlike organs donated after brain death, portal blood of DCD donors has an enhanced propensity to trigger inflammatory responses. 


\section{Aims Part II: machine perfusion}

The aim of part II of this thesis was to develop and optimise a working mechanical model for ex vivo NMP of human donor livers currently declined for transplantation. We also used this perfusion technique as a platform to study the bile ducts and to assess whether a biomarker could be found to predict the development of stricture formation.

As machine perfusion of human livers is only used in limited centres around the world, we systematically reviewed the current perfusion protocols in chapter 6 and assessed their safety and applicability.

In our centre we developed an NMP protocol, which is described in chapter 7. To date, we perfused 10 livers deemed unsuitable for transplantation. Both DBD and DBD livers were perfused and the results are discussed in Chapter 8. In this chapter we also explored the impact of machine perfusion on the development of biliary injury. As biliary injury and stricture formation is a major concern when using DCD donors, we aimed to assess if NMP would modulate the degree of injury.

In the final chapter, chapter 9, a summary of the results of this thesis is provided and their contribution to the current literature is discussed. 


\section{References}

1. Staudacher V. [Transplantation of an organ with vascular anastomoses]. Riforma Med. 1952;66(39):1060.

2. Welch CS. A note on transplantation of the whole lier in dogs. Transplant Bull. 1955(2):54-5.

3. Cannon JA. A brief report. Transplant Bull. 1956(3):7.

4. Starzl TE, Marchioro TL, Vonkaulla KN, Hermann G, Brittain RS, Waddell WR. HOMOTRANSPLANTATION OF THE LIVER IN HUMANS. Surg Gynecol Obstet. 1963;117:659-76.

5. Starzl TE, Groth CG, Brettschneider L, Penn I, Fulginiti VA, Moon JB, et al. Orthotopic homotransplantation of the human liver. Ann Surg. 1968;168(3):392-415.

6. Calne RY, Williams R. Liver transplantation in man. I. Observations on technique and organization in five cases. BMJ. 1968;4(5630):535-40.

7. Calne RY, Rolles K, White DJ, Thiru S, Evans DB, McMaster P, et al. Cyclosporin A initially as the only immunosuppressant in 34 recipients of cadaveric organs: 32 kidneys, 2 pancreases, and 2 livers. Lancet. 1979;2(8151):1033-6.

8. Starzl TE, Iwatsuki S, Shaw BW, Jr., Van Thiel DH, Gartner JC, Zitelli BJ, et al. Analysis of liver transplantation. Hepatology. 1984;4(1 Suppl):47s-9s.

9. Liver transplantation. National Institutes of Health Consensus Development. National Institutes of Health consensus development conference summary. 1983;4(7):15 p.

10. Rolles K, Williams R, Neuberger J, Calne R. The Cambridge and King's College Hospital experience of liver transplantation, 1968-1983. Hepatology. 1984;4(1 Suppl):50s-5s.

11. Pichlmayr R, Brolsch C, Wonigeit K, Neuhaus P, Siegismund S, Schmidt FW, et al. Experiences with liver transplantation in Hannover. Hepatology. 1984;4(1 Suppl):56s-60s.

12. Krom RA, Gips CH, Houthoff HJ, Newton D, van der Waaij D, Beelen J, et al. Orthotopic liver transplantation in Groningen, The Netherlands (1979-1983). Hepatology. 1984;4(1 Suppl):61s-5s.

13. Strong RW. Obstacles to the establishment of liver transplantation in Australia. J Gastroenterol Hepatol. 2009;24 Suppl 3:S119-23.

14. Strong R, Ong TH, Pillay P, Wall D, Balderson G, Lynch S. A new method of segmental orthotopic liver transplantation in children. Surgery. 1988;104(1):104-7.

15. Strong RW, Lynch SV, Ong TH, Matsunami H, Koido Y, Balderson GA. Successful liver transplantation from a living donor to her son. N Engl J Med. 1990;322(21):1505-7.

16. ANZLTR. 24th annual report Australia and New Zealand liver transplant registry. 2014.

17. Durand F, Renz JF, Alkofer B, Burra P, Clavien PA, Porte RJ, et al. Report of the Paris consensus meeting on expanded criteria donors in liver transplantation. Liver Transpl. 2008;14(12):1694-707.

18. Laing RW, Scalera I, Isaac J, Mergental H, Mirza DF, Hodson J, et al. Liver transplantation using grafts from donors after circulatory death: A propensity-matched study from a single centre. Am J Transplant. 2016.

19. Dubbeld J, Hoekstra H, Farid W, Ringers J, Porte RJ, Metselaar HJ, et al. Similar liver transplantation survival with selected cardiac death donors and brain death donors. Br J Surg. 2010;97(5):744-53.

20. Blok JJ, Detry O, Putter H, Rogiers X, Porte RJ, van Hoek B, et al. Longterm results of liver transplantation from donation after circulatory death. Liver Transpl. 2016;22(8):1107-14.

21. Vries de $\mathrm{Y}$, Buis $\mathrm{Cl}$, Mahesh SVK, Berg van den AP, Porte RJ. Non-anastomotic biliary strictures are more severe after transplantation of donation after circulatory death, compared to donation after brain death livers. HPB. 2016;18:e558-9.

22. Buis $\mathrm{Cl}$, Verdonk RC, Van der Jagt EJ, van der Hilst CS, Slooff MJ, Haagsma EB, et al. Nonanastomotic biliary strictures after liver transplantation, part 1: Radiological features and risk factors for early vs. late presentation. Liver Transpl. 2007;13(5):708-18.

23. Chang JH, Lee I, Choi MG, Han SW. Current diagnosis and treatment of benign biliary strictures after living donor liver transplantation. World J Gastroenterol. 2016;22(4):1593-606.

24. Op den Dries S, Sutton ME, Lisman T, Porte RJ. Protection of bile ducts in liver transplantation: looking beyond ischemia. Transplantation. 2011;92(4):373-9. 
25. Hoekstra H, Porte RJ, Tian Y, Jochum W, Stieger B, Moritz W, et al. Bile salt toxicity aggravates cold ischemic injury of bile ducts after liver transplantation in Mdr2+/- mice. Hepatology. 2006;43(5):102231.

26. Fickert $\mathrm{P}$, Fuchsbichler A, Wagner $\mathrm{M}$, Zollner $\mathrm{G}$, Kaser $\mathrm{A}$, Tilg $\mathrm{H}$, et al. Regurgitation of bile acids from leaky bile ducts causes sclerosing cholangitis in Mdr2 (Abcb4) knockout mice. Gastroenterology. 2004;127(1):261-74.

27. Lee NP. Hepatic tight junctions: From viral entry to cancer metastasis. World Journal of Gastroenterology. 2010;16(3):289.

28. Buis $\mathrm{Cl}$, Geuken E, Visser DS, Kuipers F, Haagsma EB, Verkade HJ, et al. Altered bile composition after liver transplantation is associated with the development of nonanastomotic biliary strictures. J Hepatol. 2009;50(1):69-79.

29. Guntaka SR, Samak G, Seth A, LaRusso NF, Rao R. Epidermal growth factor protects the apical junctional complexes from hydrogen peroxide in bile duct epithelium. Lab Invest. 2011;91(9):1396409.

30. Hanada S, Harada M, Koga H, Kawaguchi T, Taniguchi E, Kumashiro R, et al. Tumor necrosis factoralpha and interferon-gamma directly impair epithelial barrier function in cultured mouse cholangiocytes. Liver Int. 2003;23(1):3-11.

31. Sheth P, Delos Santos N, Seth A, LaRusso NF, Rao RK. Lipopolysaccharide disrupts tight junctions in cholangiocyte monolayers by a c-Src-, TLR4-, and LBP-dependent mechanism. Am J Physiol Gastrointest Liver Physiol. 2007;293(1):G308-18.

32. Brunner SM, Junger $H$, Ruemmele $P$, Schnitzbauer AA, Doenecke A, Kirchner GI, et al. Bile duct damage after cold storage of deceased donor livers predicts biliary complications after liver transplantation. J Hepatol. 2013;58(6):1133-9.

33. Carrel A, Lindbergh CA. The culture of whole organs. Science. 1935;81(2112):621-3.

34. Tolboom H, Pouw RE, Izamis ML, Milwid JM, Sharma N, Soto-Gutierrez A, et al. Recovery of warm ischemic rat liver grafts by normothermic extracorporeal perfusion. Transplantation. 2009;87(2): 170-7.

35. Brockmann J, Reddy S, Coussios C, Pigott D, Guirriero D, Hughes D, et al. Normothermic perfusion: a new paradigm for organ preservation. Ann Surg. 2009;250(1):1-6.

36. schon $\mathrm{M}$, kollmar $\mathrm{O}$, Wolf $\mathrm{S}$, Scherm H, Matthes $\mathrm{M}$, Akkoc N, et al. Liver Transplantation After Organ Preservation With Normothermic Extracorporeal Machine Perfusion. Ann Surg. 2001;233(1):114-23.

37. Tolboom H, Milwid JM, Izamis ML, Uygun K, Berthiaume F, Yarmush ML. Sequential cold storage and normothermic perfusion of the ischemic rat liver. Transplant Proc. 2008;40(5):1306-9.

38. op den Dries S, Karimian N, Sutton ME, Westerkamp AC, Nijsten MW, Gouw AS, et al. Ex vivo normothermic machine perfusion and viability testing of discarded human donor livers. Am J Transplant. 2013;13(5):1327-35 


\section{Chapter 2}

Increased liver non-use: The implications of the shift

towards Donation after Circulatory Death in

\section{Australia}

J Reiling

E Forrest

KR Bridle

L Britton

N Santrampurwala

DHG Crawford

CHC Dejong

J Fawcett

Submitted 


\section{Abstract}

\section{Background}

In recent years, more livers are being declined for transplantation in Australia. The aim of this study was to evaluate the impact of donation after cardiac death (DCD) and other factors associated with organ quality on liver utilisation rates in Australia.

\section{Method}

Data on organ donors who donated at least one organ between 2005 and 2014 were obtained from the ANZOD Registry. Temporal changes in donor characteristics were assessed and a logistical regression analysis was performed to evaluate their association with liver non-use.

\section{Key findings}

The number of organ donors increased from 163 in 2005 to 332 in 2014 with $19 \%$ being DCD donors $(p<0.001)$. The percentage of livers deemed unsuitable for transplantation increased from $26 \%$ in 2005 to $43 \%$ in $2014(p<0.001)$. DCD was identified as the most important risk factor for non-use with an OR of 24.06 (95\% Cl: 17.47-33.14), $p<0.001)$ followed by donor age, obesity and diabetes.

\section{Conclusions}

This study shows a significant decrease in liver utilization rates in Australia over a 10-year period with DCD donation as the most important independent risk factor. Novel approaches, such as machine perfusion, require further evaluation in order to be used to increase the number of livers available for transplantation. 


\section{Introduction}

A shortage of available donors remains a major limiting factor in the field of liver transplantation. Currently in Australia, only $50 \%$ of patients with end stage liver disease on the waiting list receive a life-saving transplant each year. At the same time, $10 \%$ of patients die or their condition deteriorates to the point that they are no longer deemed suitable for transplant. ${ }^{1}$ In 2008, the Australian Federal Government announced a reform program to increase the number of organ donors. As a result, organ donor rates increased from 12 to 16.1 per million population (PMP). ${ }^{2}$ Unfortunately, the number of organ donors who donated their liver has failed to increase at the same rate, suggesting that more livers are deemed unsuitable for transplantation. ${ }^{1,2}$

As well as encouraging the use of extended criteria livers (e.g. older donors and steatotic livers) and a drive to maximise splitting of suitable donor livers, one of the main strategies adopted has been the promotion of controlled donation after circulatory death (DCD, Maastricht classification type $\mathrm{II}^{3}$ ). Currently, 28\% of organ donors in Australia are DCD donors, which is low compared to the United Kingdom and the Netherlands, where $40 \%$ and $45 \%$ of organ donors are DCD respectively. ${ }^{2,4,5}$ DCD lung and kidney programs have been very successful with graft and patient survival rates comparable to conventional Donation after Brain Death (DBD) organs. ${ }^{6,7}$ Unfortunately the experience has not been the same for livers. The use of DCD livers has been associated with inferior graft quality and the development of nonanastomotic strictures (NAS) in 9-31\% of recipients. ${ }^{8,9}$ As a result, the percentage of liver non-use attributable to DCD has increased in the US from $9 \%$ in 2004 to $28 \%$ in $2010 .^{10}$ It is currently unclear what effect DCD donation has on liver non-use in Australia.

DCD livers, severely steatotic livers and livers from older donors are more susceptible to ischaemia-reperfusion injury. ${ }^{11,12}$ Alternative preservation methods such as ex-vivo machine perfusion have therefore been proposed in order to better preserve organ quality. Hypothermic machine perfusion at $4-10^{\circ} \mathrm{C}$ as well as normothermic machine perfusion using a blood based perfusate have proven to be safe and technically feasible in small clinical series and results are pending from the first randomised controlled trials. $^{13-15}$ If extended criteria donor livers could be safely used following machine perfusion without increasing recipient morbidity, this could consequentially increase the number of donor livers available for transplantation. ${ }^{16}$

The aim of this study was to evaluate the impact of DCD and other donor characteristics associated with more marginal donors on liver non-use in Australia over the past 10 years. The study also aimed to determine the number of DCD livers that would be suitable candidates for machine perfusion if this technique could be implemented. 


\section{Methods}

\section{Study population}

All adult organ donors in Australia who donated at least one organ between 1st January 2005 and 1st of January 2015 were included in this study. As these donors had no absolute contraindication for the organ donation process, unsuitability for transplantation was therefore most likely the result of liver specific reasons. A liver donor was defined as an organ donor from whom the liver was retrieved and subsequently successfully transplanted into a recipient. Donors of split liver grafts $(n=134)$ were excluded as they represent a specific subset of high quality donors and their characteristics differ from whole liver donors. De-identified donor data was obtained from the Australia and New Zealand Organ Donor (ANZOD) registry. In one Australian jurisdiction, the state of Queensland, approval was obtained from the human research ethics committee of the Princess Alexandra Hospital as well as the University of Queensland to access confidential donor data. This allowed access to donor files when data was incomplete or not available from the organ donor registry.

\section{Data collection}

Donor demographics associated with marginal grafts such as donor age, body mass index (BMI), donation type (DCD or DBD), hepatitis B (hepatitis B surface antigen positive) or hepatitis $C$ infection (hepatitis $C$ (HCV) antibody positive and presence of HCV RNA by nucleic acid testing), hypertension and diabetes mellitus (DM) were collected. As donor age was not normally distributed, the following age categories were used: $<40,40-49,50-59$ and $>60$ years. BMI was classified as underweight $(<18.5$ $\left.\mathrm{kg} / \mathrm{m}^{2}\right)$, normal weight $\left(18.5-24.99 \mathrm{~kg} / \mathrm{m}^{2}\right)$, overweight $\left(25-29.99 \mathrm{~kg} / \mathrm{m}^{2}\right)$ and obese $\left(>30 \mathrm{~kg} / \mathrm{m}^{2}\right.$ ). Liver biopsy results were not included from any of the donors, as it is not routine practice to perform a biopsy and the inclusion of biopsy results of selected donors would bias the results. Due to incomplete data in the organ donor registry during this period, it was not possible to include liver function test results as a parameter in the main analyses. Instead, a sub analysis was performed of the Queensland state donors $(n=432)$ where alanine aminotransferase (ALT) was categorised as $<100$ versus $>100 \mathrm{U} / \mathrm{L}$, bilirubin as $<20$ versus $>20 \mu \mathrm{mol} / \mathrm{L}$ and $\gamma$ glutamyl transferase as $<40$ versus $>40 \mathrm{U} / \mathrm{L}$.

Additional factors that potentially influence the decision to use a liver for donation such as gender, blood group, presence of hepatitis B core antibody, year of organ retrieval, state of organ retrieval, cause of death and smoking status were also recorded. If a donor was identified as a DCD donor, warm ischaemia time (WIT) prior to the start of cold perfusion was recorded. The start of this period was marked by a systolic blood pressure lower than $50 \mathrm{mmHg}$ or oxygen saturation lower than $50 \%$, 
whichever occurred first. In Australia, livers from DCD donors are generally considered for transplantation if the warm ischaemic time is limited to 30 minutes and they do not have any other extended criteria beyond DCD. ${ }^{17}$ From every donor, data on the organs retrieved and whether they were used or discarded was recorded (kidneys, liver, lung, heart, and pancreas). Lastly, from all donors in the State of Queensland, the distance between donor and recipient hospital was recorded. The state area comprises $1,727,000 \mathrm{~km}^{2}$, with the liver transplantation centre located in the far south-east corner about $1700 \mathrm{~km}$ from the furthest donor hospital. For the purpose of this study travel distance was defined as metropolitan $(<100 \mathrm{~km})$ and nonmetropolitan ( $>100 \mathrm{~km}$ ). Overall each variable of interest was available from $>95 \%$ of organ donors unless stated otherwise.

\section{Impact of machine perfusion in the state of Queensland}

To determine likely candidates for machine perfusion, those that would fulfil current criteria for transplantation were identified from the United Kingdom and the Netherlands, as both these countries have very successful DCD liver transplant programs. ${ }^{8,9}$ Following review of current guidelines and recently published papers on the use of DCD donors in these countries, the DCD donor cohort was stratified by age, warm ischaemic time and BMI (as a surrogate marker for steatosis grade). ${ }^{18,19}$ Length of stay in the intensive care unit and cold ischaemic time are additional components that define marginality in the United Kingdom, however these parameters were not available in the present cohort. Instead, the distance between donor and recipient hospital was recorded and was used as an indicator of expected cold ischaemic time. Because of completeness of data, the focus was on organ donors in one jurisdiction, the state of Queensland.

\section{Statistical analysis}

All continuous variables are expressed as median (interquartile range) and all categorical variables as frequency (percentage). A chi-squared test was performed to compare categorical variables whereas a Mann-Whitney $U$ test was used to compare all continuous variables. A multivariable logistical regression analysis was performed to identify donor characteristics associated with liver non-use controlled for all factors with a $p$-value $<0.1$ in univariable analysis.

A sub-analysis was performed on all Queensland donors to assess the association between donor hospital distance, abnormal liver function tests and liver non-use. The statistical analysis was performed using IBM SPSS Statistics for Macintosh, Version 23.0 (IBM Corp. IMB SPSS statistics, Armonk, NY) and a $p$-value of $<0.05$ was considered significant. 


\section{Results}

\section{Donor characteristics}

Between 2005 and 2014 a total of 2413 organ donors in Australia were identified who have donated at least a single organ (Table 2.1). Of these, the majority of organ donors were Caucasian (93\%) and 55\% were male. In addition, 401 (16\%) were older than 60 years old, 468 (19\%) were DCD, and 900 (37\%) and 607 (25\%) were overweight or obese respectively. Only a very small proportion of organ donors tested positive for hepatitis $\mathrm{B}$ or hepatitis $\mathrm{C}$ ( $0.3 \%$ and $1 \%$ respectively), $8 \%$ were diabetic and $28 \%$ had a history of hypertension. Stroke was the most common cause of death (54\%) followed by hypoxia. The median number of organs transplanted per donor was three. The majority of organ donors donated one or both kidneys (95\%) followed by liver (63\%), lung (42\%), pancreas (31\%) and heart (25\%).

Table 2.1 Donor characteristics between 2005-2014.

\begin{tabular}{|c|c|c|c|c|}
\hline Characteristic & $2005(\mathrm{~N}=163)$ & $2014(\mathrm{~N}=332)$ & Overall $(\mathrm{N}=2413)$ & $p$-value \\
\hline Age (years) & $48(37-57)$ & $52(41-60)$ & $50(37-57)$ & 0.008 \\
\hline Gender (male) & $85(52 \%)$ & $183(55 \%)$ & $1314(55 \%)$ & 0.5 \\
\hline Race (Caucasian) & $154(95 \%)$ & 396 (89\%) & 2237 (93\%) & 0.05 \\
\hline Body mass index $\left(\mathrm{kg} / \mathrm{m}^{2}\right)$ & $27(24-30)$ & $27(24-30)$ & $26(24-30)$ & 0.8 \\
\hline Donor type (DBD) & 155 (95\%) & 237 (71\%) & 1945 (81\%) & $<0.001$ \\
\hline \multicolumn{5}{|l|}{ Cause of Death } \\
\hline Stroke & 100 (61\%) & $171(52 \%)$ & $1310(54 \%)$ & $<0.001$ \\
\hline Hypoxia & $17(10 \%)$ & $94(28 \%)$ & $502(21 \%)$ & \\
\hline Accident & $25(15 \%)$ & $46(14 \%)$ & $368(15 \%)$ & \\
\hline Other & $21(13 \%)$ & $21(6 \%)$ & $233(10 \%)$ & \\
\hline Diabetes mellitus & $12(8 \%)$ & $21(6 \%)$ & $199(8 \%)$ & 0.5 \\
\hline Hypertension & $39(24 \%)$ & 96 (29\%) & $669(28 \%)$ & 0.4 \\
\hline Smoking & & & & 0.01 \\
\hline Current & $57(35 \%)$ & 145 (44\%) & 969 (40\%) & \\
\hline Never & $74(45 \%)$ & $105(32 \%)$ & $855(35 \%)$ & \\
\hline Former & $32(20 \%)$ & $82(25 \%)$ & $583(24 \%)$ & \\
\hline Hepatitis B core antibody & $11(7 \%)$ & $18(5 \%)$ & $128(5 \%)$ & 0.02 \\
\hline Hepatitis B surface antigen & $0(0 \%)$ & $5(2 \%)$ & $7(0.3 \%)$ & 0.2 \\
\hline Hepatitis C antibody + HCV RNA & $3(2 \%)$ & $2(1 \%)$ & $21(1 \%)$ & 0.2 \\
\hline WIT (min, 400/468) & Data not available & $23(20-28)$ & $22(18-27)$ & - \\
\hline $\operatorname{ALT}^{\#}(\mathrm{U} / \mathrm{L})$ & $28(16-85)$ & $42(23-68)$ & $38(21-71)$ & 0.5 \\
\hline Bilirubin ${ }^{\#}(\mu \mathrm{mol} / \mathrm{L})$ & $13(7-16)$ & $13(9-18)$ & $12(8-17)$ & 0.4 \\
\hline Gamma-GT" (U/L) & $41(22-75)$ & $32(19-59)$ & $43(23-71)$ & 0.4 \\
\hline Distance donor hospital ${ }^{\#}(\mathrm{Km})$ & $37(0-870)$ & $67(8-619)$ & $37(8-122)$ & 0.4 \\
\hline Nr organs retrieved and transplanted & $4(3-5)$ & $3(2-4)$ & $3(2-4)$ & $<0.001$ \\
\hline
\end{tabular}

Note: Because of rounding not all percentages add up to $100 \%$. \# The data presented here is from the Queensland state donor subgroup only. $\mathrm{N}=432$. HCV: hepatitis $\mathrm{C}$ virus. 


\section{Changes in donor characteristics over time}

Over the 10-year study period, the number of organ donors per year more than doubled from 163 in 2005 to 332 in 2014 (Figure 2.1). Since 2008 there has been a steady increase in the number of DCD organ donors with $29 \%$ of all organs donated being from a DCD donor in 2014. In 2005 the percentage of organ donors younger than 40 years old was $29 \%$, which decreased to $22 \%$ in 2014 . In contrast, the proportion of organ donors older than 60 increased by $11 \%$. During the study period, the proportion of overweight and obese donors remained stable with a median BMI of $26(24-30) \mathrm{kg} / \mathrm{m}^{2}$.

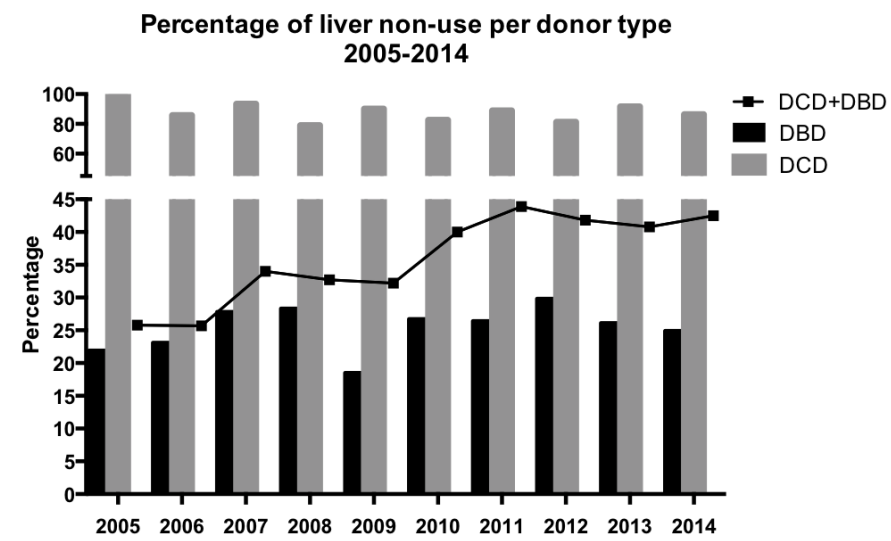

Figure 2.1 Increased percentage of liver non-use between 2005 and 2014. This graph shows DCD (grey bars) and DBD (black bars) non-liver donors as a percentage of all organ donors from the corresponding donor type. The black line represents the overall percentage of livers not used for transplantation when the two donor types were combined.

More donors were current or former smokers, or died from hypoxic injury in 2014 compared with 2005 (Table 2.1). Overall the median number of organs retrieved per donor decreased from $4(3-5)$ in 2005 to $3(2-4)$ in $2014(p<0.001)$. Among Queensland state organ donors, the median ALT, bilirubin and $\gamma$-glutamyl transferase values did not change over the 10-year study period with 71 (16\%) organ donors having an ALT >100 U/L.

\section{Temporal trends in liver non-use}

Overall, a liver was retrieved and subsequently transplanted from 1509 (63\%) organ donors. Over time, the proportion of livers deemed unsuitable for transplantation from DBD donors remained stable between 20-30\% (Figure 2.2). Since 2005, only $62(13 \%)$ DCD livers were used for transplantation. Over the 10 -year study period, the 
percentage of DCD livers rejected for transplantation was between $80 \%$ and $100 \%$. As the proportion of DCD donors has increased over time, the overall number of livers not used for transplantation increased from 42 (26\%) in 2005 to 141 (43\%) in 2014 $(p<0.001)$.

A

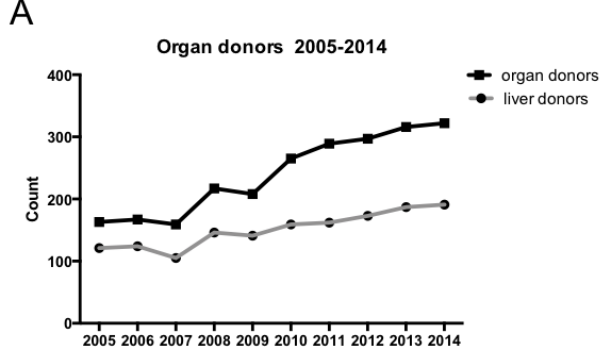

C

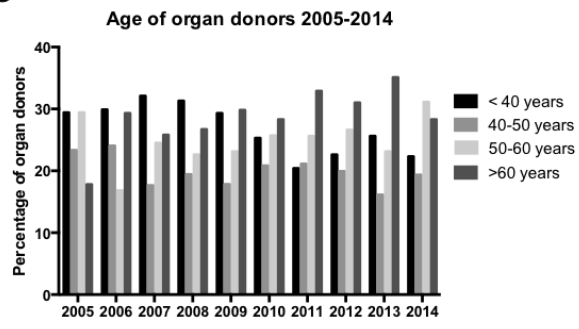

B

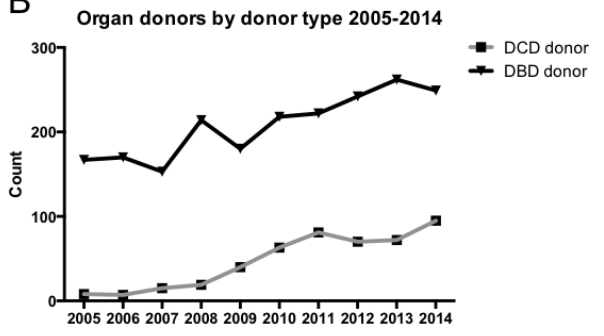

D BMI of organ donors 2005-2014

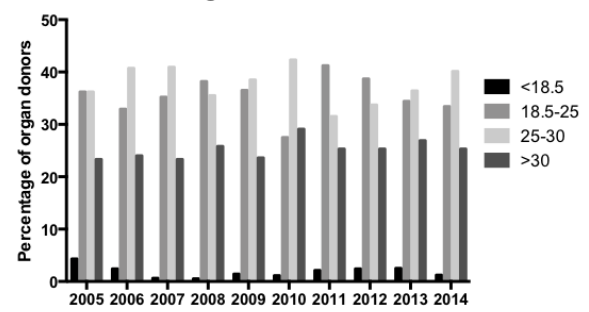

Figure 2.2 Changing organ donor characteristics between 2005 and 2014. A) Increase in the number of organ donors and organ donors donating their liver (liver donors) between 2005 and 2014. B) Number of livers donated after circulatory death (DCD) and donated after brain death (DBD) between 2005 and 2014. C) Age of organ donors between 2005 and 2014. D) Body mass index (BMI) of organ donors between 2005 and 2014.

\section{Risk factors for liver non-use}

In univariable analysis, liver non-use was strongly associated with DCD donor type $(p<0.001$, Table 2.2). Furthermore, livers from older donors and overweight or obese donors were less likely to be used. Organs from donors who died from hypoxia, compared to those who suffered a stroke, were more likely to be declined for transplant. In addition, liver non-use was associated with donors who were former smokers, compared to those who did not smoke. The presence of hypertension and diabetes in the donor population were also associated with liver non-use. In the subgroup of 468 DCD donors, a WIT over 20 minutes was associated with liver nonuse (OR 3.10 (95\% Cl: 1.70-5.61), p<0.001). In univariable analysis of Queensland state organ donors, ALT concentration over $100 \mathrm{U} / \mathrm{L}$ and $\gamma$-glutamyl transferase greater than $40 \mathrm{U} / \mathrm{L}$ were associated with liver non-use (OR 3.04 (95\% Cl: $1.80-5.14$ ) $p=0.029$ and OR 12.20 (95\% Cl: 7.290-20.42) $p<0.001$ respectively). 
Table 2.2 Donor Characteristics associated with liver non-use.

\begin{tabular}{lcccc}
\hline Characteristic & \multicolumn{2}{c}{ Univariable regression } & \multicolumn{2}{c}{ Multivariable regression* } \\
\cline { 2 - 5 } & Odds ratio $(95 \% \mathrm{Cl})$ & $p$-value & Odds ratio $(95 \% \mathrm{Cl})$ & $p$ - value \\
\hline Age (versus $<40$ years) & & $<0.001$ & & $<.001$ \\
$40-50$ years & $1.54(1.19-2.00)$ & 0.001 & $1.57(1.13-2.19)$ & 0.008 \\
$50-60$ years & $1.96(1.54-2.50)$ & $<0.001$ & $2.43(1.48-2.83)$ & $<0.001$ \\
$>60$ Years & $2.32(1.84-2.92)$ & $<0.001$ & $3.10(2.21-4.34)$ & $<0.001$ \\
BMI (versus 18.5-24.99 kg/m²) & & $<0.001$ & & $<0.001$ \\
$<18.5 \mathrm{~kg} / \mathrm{m}^{2}$ & $1.51(0.81-2.83)$ & 0.2 & $1.82(0.86-3.85)$ & 0.1 \\
$25-29.99 \mathrm{~kg} / \mathrm{m}^{2}$ & $1.32(1.09-1.62)$ & 0.007 & $1.28(1.00-1.64)$ & 0.05 \\
$>30 \mathrm{~kg} / \mathrm{m}^{2}$ & $2.57(2.07-3.19)$ & $<0.001$ & $2.90(2.22-3.80)$ & $<0.001$ \\
Donor type (DCD) & $19.03(14.30-25.33)$ & $<0.001$ & $24.06(17.47-33.14)$ & $<0.001$ \\
Cause of death (versus stroke) & & $<0.001$ & & 0.35 \\
Hypoxia & $1.75(1.42-2.15)$ & $<0.001$ & $1.27(0.96-1.69)$ & 0.1 \\
Accident & $1.02(0.80-1.30)$ & 0.9 & $0.99(0.71-1.37)$ & 0.9 \\
Other & $1.09(0.82-1.46)$ & 0.5 & $1.14(0.77-1.67)$ & 0.5 \\
Hypertension & $1.54(1.29-1.86)$ & $<0.001$ & $1.21(0.95-1.54)$ & 0.1 \\
Diabetes mellitus & $1.69(1.27-2.26)$ & $<0.001$ & $1.60(1.13-2.26)$ & 0.009 \\
Smoking (versus never) & & $<0.001$ & & 0.2 \\
Current & $0.72(0.59-0.87)$ & 0.001 & $0.80(0.63-1.02)$ & 0.07 \\
Former & $1.10(0.89-1.37)$ & 0.4 & $0.95(0.73-1.23)$ & 0.7 \\
Hepatitis B core antibody & $1.04(0.72-1.51)$ & 0.8 & & 0.31 \\
Hepatitis B surface antigen & $4.19(0.81-21.67)$ & 0.09 & $2.53(0.42-15.10)$ & 0.2 \\
Hepatitis C antibody + HCV RNA & $0.52(0.19-1.42)$ & 0.2 & & \\
\hline
\end{tabular}

*All factors with a $P$ value $<0.1$ in univariable regression analysis as well as $A B O$ blood group, donor state, year (continuous), race and gender were included in the multivariable regression model.

In multivariable analysis, DCD donor type was identified as the most significant independent risk factor for liver non-use with an odds ratio of $24.06(95 \% \mathrm{Cl}$ : 17.47-33.14) $(p<0.001$, Table 2.2). Furthermore, donor age over 40 years was associated with liver non-use with livers from donors over 60 years of age being the least likely to be used (OR 3.10 ( $95 \% \mathrm{Cl}: 2.21-4.34), p<0.001)$. Donor obesity was also associated with higher odds for non-use $(p<0.001)$ as was diabetes. In the Queensland state organ donor subset, ALT $>100 \mathrm{U} / \mathrm{L}$ and Gamma-GT $>40 \mathrm{U} / \mathrm{L}$ were strongly associated with non-use (OR 4.43 (95\% Cl: 1.98-9.93) $p<0.001$ and OR 11.90 (95\% Cl: 5.97-23.76) $p<0.001$ respectively).

\section{Characteristics of DCD non-liver donors in the state of Queensland}

Since 2008, there were 90 DCD organ donors in the state of Queensland but only 9 livers retrieved from these donors were used for transplantation. The non-liver donors were categorised by duration of warm ischaemia, age and BMI category to identify livers that could have been transplanted had some effective method, such as normothermic machine perfusion been available to better assess their suitability. Furthermore the distance between the donor hospital and the liver transplant centre was assessed. Compared to DBD donors, DCD donors were more often in the 
metropolitan area ( $86 \%$ versus $66 \%, \mathrm{p}<0.001$ ). As can be seen in Table 2.3 , the majority of DCD non-liver donors (85\%) had a WIT of less than 30 minutes and 27 (33\%) had recorded WIT less than 20 minutes. Only seven (9\%) donors fulfilled all three optimal donor criteria (BMI $<25 \mathrm{~kg} / \mathrm{m}^{2}$, WIT $<20$ minutes, age $<50$ years). All these donors were from hospitals in the metropolitan area. An additional 18 (22\%) donors had one adverse parameter (age $50-60$ years, BMI $25-30 \mathrm{~kg} / \mathrm{m}^{2}$ or WIT 20-30 minutes) three of which were non-metropolitan donors. A further eight (10\%) donors had two adverse parameters and were within a $100 \mathrm{~km}$ from the liver transplant centre.

Table 2.3 Number of DCD non-liver donors in Queensland by age category, body mass index and warm ischaemic time.

\begin{tabular}{llllll}
\hline DCD donors $N=81$ & & \multicolumn{5}{l}{ Donor age (years) } \\
\cline { 3 - 6 } & Body mass index & $<40$ & $40-50$ & $50-60$ & $>60$ \\
\hline WIT<20 minutes & $<25 \mathrm{~kg} / \mathrm{m}^{2}$ & $5(6 \%)$ & $2(2 \%)$ & $3(4 \%)$ & $1(1 \%)$ \\
N=27 (33\%) & $25-30 \mathrm{~kg} / \mathrm{m}^{2}$ & $2(2 \%)$ & $4(5 \%)$ & $2(2 \%)$ & $2(2 \%)$ \\
WIT 20-30 minutes & $>30 \mathrm{~kg} / \mathrm{m}^{2}$ & $2(2 \%)$ & $1(0 \%)$ & $2(2 \%)$ & $1(1 \%)$ \\
N=41 (51\%) & $25 \mathrm{~kg} / \mathrm{m}^{2}$ & $5(6 \%)$ & $4(5 \%)$ & $3(4 \%)$ & $3(4 \%)$ \\
& $25-30 \mathrm{~kg} / \mathrm{m}^{2}$ & $3(4 \%)$ & $0(0 \%)$ & $9(11 \%)$ & $5(6 \%)$ \\
WIT>30 minutes & $>30 \mathrm{~kg} / \mathrm{m}^{2}$ & $1(1 \%)$ & $1(1 \%)$ & $4(5 \%)$ & $3(4 \%)$ \\
N=13 (16\%) & $<25 \mathrm{~kg} / \mathrm{m}^{2}$ & $0(0 \%)$ & $4(5 \%)$ & $1(1 \%)$ & $1(1 \%)$ \\
& $25-30 \mathrm{~kg} / \mathrm{m}^{2}$ & $0(0 \%)$ & $1(1 \%)$ & $1(1 \%)$ & $1(1 \%)$ \\
& $>30 \mathrm{~kg} / \mathrm{m}^{2}$ & $0(0 \%)$ & $1(1 \%)$ & $3(4 \%)$ & $0(0 \%)$ \\
\hline
\end{tabular}

WIT: warm ischaemic time

\section{Discussion}

In recent years, DCD donors have been identified as a potential source to increase donor numbers. In the United Kingdom and the Netherlands, over one third of all organ donors are DCD donors and these countries have successfully implemented the use of DCD livers with 1-year graft and patient survival similar to conventional DBD donors. ${ }^{8,9}$ Unfortunately the Australian experience is rather different. Despite the fact that $468(19 \%)$ organ donors in the present cohort were DCD, only 62 livers were retrieved and subsequently transplanted. This contributed to the significant rise in liver non-use rates from $26 \%$ in 2005 to $43 \%$ in 2014.

The decreasing rate of liver utilisation is a pressing problem around the globe. In the United Kingdom, the proportion of livers used for transplantation has dropped from $601(79 \%)$ in 2005-06 to $812(63 \%)$ in 2014-15., ${ }^{5,20}$ Furthermore, Orman et al. ${ }^{10}$ found that in the US, liver non-use increased from $15 \%$ in 2004 to $21 \%$ in 2010 with DCD as the most important risk factor (OR 21.31 (95\% Cl: 18.30-24.81)). The present study indicates that liver non-use is an even bigger problem in Australia, as the proportion of DCD donors in the total cohort is higher (19\% versus 9\%). Australian centres have 
had a conservative approach to the use of DCD livers mainly due to a small increased risk of primary non-function that has been observed in our country (Jan 2006- Dec 2015: DBD; 20 (0.9\%), DCD; 5 (5.1\%), $p<0.01$, unpublished data, courtesy of Glenda Balderson, ANTLR) as well as NAS formation (21). The low population density (3.1 people $\left./ \mathrm{km}^{2}\right)^{22}$ and the large distances between the five transplant centres (up to $3600 \mathrm{Km}$ ) significantly reduce the chances of finding a new liver in case of primary non function of the graft. Therefore, livers from DCD donors are rarely considered for transplantation if they are aged over $40-45$, in an attempt to select more superior grafts. $^{17}$

One of the most important questions remains is if some DCD donors, if given the time, would in fact progress to brain death. ${ }^{23}$ If so, this would mean that a proportion of DBD organ donors, with expected good organ quality, were converted into the less favourable DCD type, which the present study shows is associated with high odds of liver non-use. Reasons for this conversion could be that the DCD donation process is easier to comprehend by donor families and they prefer to accompany their family member until cardiac arrest has occurred. ${ }^{24}$ Furthermore, bed capacity in the intensive care unit (ICU) could put pressure on the time-consuming neurological observations required to medically and legally determine brain death. A recent study assessing transplant rates in 82 countries showed that between 2000 and 2010, countries with high DCD rates had declining or static DBD rates. ${ }^{23}$ Furthermore, this study by Bendorf et al. showed that the increase in the number of DBD donors, and not DCD, was the basis of higher sustained donation rates $>20$ PMP in countries such as Spain, France and the USA. Taken together, this might indicate that the focus needs to shift back to increasing the number of DBD donors.

To facilitate the use of high-risk donors such as DCD, modifiable risk factors such as WIT, cold ischaemic time (CIT) and distance travelled need to be modified. Currently in Australia, withdrawal of life support takes place in the ICU. Following cessation of circulation, a legally obligated 5-minute stand down period is in place to make sure that auto-resuscitation does not occur. The organ donor is then brought into the operating room, which can take 3-4 minutes. Withdrawal of life support in the operating room would significantly expedite the process and reduce WIT. CIT in Australia is largely dependent on travel time due to the large distances. Although actual flying times to distant parts of the state may only take 3-4 hours, ground transport between hospitals and airport has to be taken into account. Additional staff at the donor hospital (e.g. donation specialist nurse) to care for the donor once organ retrieval is finalised could facilitate a quicker departure. This approach has been implemented in the larger non-metropolitan hospitals throughout Australia. Furthermore, police escorts to and from the airport could significantly reduce travel time, especially during peak hour. Unfortunately this is not possible in most states. By far the most effective way to reduce travel time is to focus on metropolitan donors only. This approach has facilitated the use of more high-risk donors in other countries 
such as Canada. ${ }^{25}$ Nevertheless, the biggest challenge to date is to build the confidence amongst transplant surgeons and physicians that the use of DCD donor livers can be safe.

One possible way to safely increase the number of livers suitable for transplantation could be the use of alternative preservation methods such as machine perfusion. ${ }^{16}$ Machine perfusion reduces the preservation injury and therefore holds the potential to better preserve organ quality and possibly even rescue marginal donors who are outside current criteria (e.g. DCD donor organs with WIT >30 minutes and macrovesicular steatosis $>30 \%$ ). ${ }^{26-28}$ Machine perfusion furthermore opens up the possibility for organ sharing and utilisation of marginal donors between geographically distant centres as preservation periods could be extended. Lastly, normothermic machine perfusion allows for assessment of viability, an important tool to avoid transplanting a liver that would in fact fail to function. ${ }^{29}$

The unit in the state of Queensland rarely uses DCD livers and has therefore explored the use of normothermic machine perfusion. ${ }^{30}$ When analysing the present cohort, 33 DCD donors (41\%) were identified who either had nil, one or two marginal donor characteristics. When implementing machine perfusion, the use of these grafts would be the most logical first step. Obviously recipient selection to minimise the risk of adverse graft outcome is crucial. ${ }^{9,31}$

As the application of machine perfusion is still an upcoming new technique, it is hard to predict what the impact would be on the overall number of livers available for transplantation. Sutton et al. estimated that $50 \%$ of the discarded extended criteria livers (10 DCD, 2 DBD) they perfused showed signs of good organ function. ${ }^{32}$ Furthermore, based on results of a pilot series, Mergental et al. estimated a potential increase in the number of liver grafts in the United Kingdom by $15 \%$ if $70 \%$ of currently declined extended criteria livers could be used. ${ }^{33}$

The limitations of the study arise mainly from its retrospective nature. The reasons for non-use of each organ are registered in the database, but in the majority of cases this was not well defined (e.g. 'medical unsuitability' or 'DCD'). Furthermore, no histological data were available to determine the impact of hepatic steatosis. Obesity and DM were however included in the cohort and these factors are strongly associated with hepatic steatosis. ${ }^{34}$ Finally it was only possible to assess the impact of abnormal liver function tests and donor hospital location in the Queensland state donor cohort. As this cohort only comprised $18 \%$ of all Australian organ donors, it might not represent the entire study cohort.

Despite these limitations, this study confirms and reiterates previous reports of a decrease in liver utilisation with DCD donation as the most important independent risk factor. Despite comparable graft and patient outcomes to DBD donors in selected centres, DCD livers are reluctantly used in Australia. Machine perfusion, by way of better preservation and viability testing, will likely play an important role in efforts to expand the donor pool and decrease waitlist mortality. 


\section{References}

1. ANZLTR. 24th annual report Australia and New Zealand liver transplant registry. 2014.

2. Australian organ and tissue donation authority. Annual Report. 2014-2015.

3. Kootstra G, Daemen JH, Oomen AP. Categories of non-heart-beating donors. Transplant Proc. 1995;27(5):2893-4.

4. Nederlandse Transplantatie Stichting. Jaarverslag 2014. 2014.

5. NHS Blood and Transplant. Organ Donation and Transplantation Activity Report 2014/15. 2014-2015.

6. Machuca TN, Mercier O, Collaud S, Tikkanen J, Krueger T, Yeung JC, et al. Lung transplantation with donation after circulatory determination of death donors and the impact of ex vivo lung perfusion. Am J Transplant. 2015;15(4):993-1002.

7. Summers DM, Watson CJ, Pettigrew GJ, Johnson RJ, Collett D, Neuberger JM, et al. Kidney donation after circulatory death (DCD): state of the art. Kidney Int. 2015;88(2):241-9.

8. Dubbeld J, Hoekstra H, Farid W, Ringers J, Porte RJ, Metselaar HJ, et al. Similar liver transplantation survival with selected cardiac death donors and brain death donors. Br J Surg. 2010;97(5):744-53.

9. Laing RW, Scalera I, Isaac J, Mergental H, Mirza DF, Hodson J, et al. Liver transplantation using grafts from donors after circulatory death: A propensity-matched study from a single centre. Am J Transplant. 2016.

10. Orman ES, Barritt ASt, Wheeler SB, Hayashi PH. Declining liver utilization for transplantation in the United States and the impact of donation after cardiac death. Liver Transpl. 2013;19(1):59-68.

11. Monbaliu D, Liu Q, Vekemans K, Roskams T, Pirenne J. Potentiation of adverse effects of cold by warm ischemia in circulatory death donors for porcine liver transplantation. Transplant Proc. 2012;44(9): 2874-9.

12. Berthiaume F, Barbe L, Mokuno Y, MacDonald AD, Jindal R, Yarmush ML. Steatosis reversibly increases hepatocyte sensitivity to hypoxia-reoxygenation injury. J Surg Res. 2009;152(1):54-60.

13. Guarrera JV, Henry SD, Samstein B, Reznik E, Musat C, Lukose TI, et al. Hypothermic machine preservation facilitates successful transplantation of "orphan" extended criteria donor livers. Am J Transplant. 2015;15(1):161-9.

14. Ravikumar R, Jassem W, Mergental H, Heaton N, Mirza D, Perera MT, et al. Liver transplantation after ex vivo normothermic machine preservation: a Phase 1 (first-in-man) clinical trial. Am J Transplant. 2016;16(6):1779-87.

15. Dutkowski P, Polak WG, Muiesan P, Schlegel A, Verhoeven CJ, Scalera I, et al. First Comparison of Hypothermic Oxygenated PErfusion Versus Static Cold Storage of Human Donation After Cardiac Death Liver Transplants: An International-matched Case Analysis. Ann Surg. 2015;262(5):764-71.

16. Orman ES, Mayorga ME, Wheeler SB, Townsley RM, Toro-Diaz HH, Hayashi PH, et al. Declining liver graft quality threatens the future of liver transplantation in the United States. Liver Transpl. 2015;21(8):1040-50.

17. van der Stelt JM, Verran DJ, deRoo RA, Christine H, Crawford M. Initial outcomes of using allografts from donation after cardiac death donors for liver transplantation in New South Wales. Med J Aust. 2013;199(2):104-6.

18. Working Party of The British Transplantation Society. United Kingdom Guidelines, Transplantation From Donors After Deceased Circulatory Death. 2013.

19. De Nederlandse Transplantatie Stichting. Modelprotocol postmortale orgaan- en weefseldonatie. 2015.

20. NHS Blood and Transplant. Transplant activity in the UK 2005-2006. 2005-2006.

21. Jay CL, Lyuksemburg V, Ladner DP, Wang E, Caicedo JC, Holl JL, et al. Ischemic cholangiopathy after controlled donation after cardiac death liver transplantation: a meta-analysis. Ann Surg. 2011;253(2): 259-64.

22. Australian Bureau of Statistics. 3218.0 Regional Population Growth, Australia 2014-15 2014-2015. Available from: http://www.abs.gov.au/AUSSTATS/abs@.nsf/Latestproducts/ 3218.0Main\%20Features152014-15 opendocument $\&$ tabname=Summary $\&$ prodno=3218.0 \&issue $=$ 2014-15\&num=\&view=. 
23. Bendorf A, Kelly PJ, Kerridge IH, McCaughan GW, Myerson B, Stewart C, et al. An international comparison of the effect of policy shifts to organ donation following cardiocirculatory death (DCD) on donation rates after brain death (DBD) and transplantation rates. PLoS One. 2013;8(5):e62010.

24. Sque $M$, Long $T$, Payne $S$. Organ donation: key factors influencing families' decision-making. Transplant Proc. 2005;37(2):543-6.

25. Sela N, Croome KP, Chandok N, Marotta P, Wall W, Hernandez-Alejandro R. Changing donor characteristics in liver transplantation over the last 10 years in Canada. Liver Transpl. 2013;19(11): 1236-44.

26. Xu H, Berendsen T, Kim K, Soto-Gutierrez A, Bertheium F, Yarmush ML, et al. Excorporeal normothermic machine perfusion resuscitates pig DCD livers with extended warm ischemia. J Surg Res. 2012;173(2):e83-8.

27. Henry SD, Nachber E, Tulipan J, Stone J, Bae C, Reznik L, et al. Hypothermic machine preservation reduces molecular markers of ischemia/reperfusion injury in human liver transplantation. Am J Transplant. 2012;12(9):2477-86.

28. Jamieson RW, Zilvetti M, Roy D, Hughes D, Morovat A, Coussios CC, et al. Hepatic steatosis and normothermic perfusion-preliminary experiments in a porcine model. Transplantation. 2011;92(3): 289-95.

29. Op den Dries S, Karimian N, Porte RJ. Normothermic machine perfusion of discarded liver grafts. Am J Transplant. 2013;13(9):2504.

30. Reiling J, Lockwood DS, Simpson AH, Campbell CM, Bridle KR, Santrampurwala N, et al. Urea production during normothermic machine perfusion: Price of success? Liver Transpl. 2015;21(5): 700-3.

31. Dutkowski P, Schlegel A, Slankamenac K, Oberkofler CE, Adam R, Burroughs AK, et al. The use of fatty liver grafts in modern allocation systems: risk assessment by the balance of risk (BAR) score. Ann Surg. 2012;256(5):861-8; discussion 8-9.

32. Sutton ME, Op Den Dries S, Karimian N, De Boer MT, Wiersema-Buist J, Gouw ASH, et al. Criteria for viability assessment of discarded human donor livers during ex-vivo normothermic machine perfusion. Liver Transplantation. 2014;20:S240.

33. Mergental H, Perera M, Laing RW, Muiesan P, Isaac JR, Smith A, et al. Transplantation of declined liver allografts following normothermic ex-situ evaluation. Am J Transplant. 2016;16(11):3235-45.

34. Williams CD, Stengel J, Asike MI, Torres DM, Shaw J, Contreras M, et al. Prevalence of nonalcoholic fatty liver disease and nonalcoholic steatohepatitis among a largely middle-aged population utilizing ultrasound and liver biopsy: a prospective study. Gastroenterology. 2011;140(1):124-31. 


\section{Chapter 3}

\section{Low dose lipopolysaccharide causes biliary injury by \\ blood biliary barrier impairment in a rat hepatic}

ischaemia-reperfusion model

J Reiling

KR Bridle

$M$ Gijbels

FG Schaap

L Jaskowski

N Santrampurwala

L Britton

CM Campbell

SWM Olde Damink

DHG Crawford

CHC Dejong

J Fawcett

Liver Transplantation, 2016 Nov 23. doi: 10.1002/It.24681 


\section{Abstract}

\section{Background}

This study explored whether bacterial endotoxins, in the form of lipopolysaccharides (LPS), could have an injurious effect on the biliary tract in conjunction with ischemia.

\section{Method}

Sixty-four rats were randomly assigned to four groups: sham operation (sham), $1 \mathrm{mg} / \mathrm{kg}$ LPS i.p. (LPS), hepatic ischemia reperfusion (IR), and IR combined with LPS (IR+LPS). Following 1 or 6 hours of reperfusion, serum liver tests, bile duct histology, immunofluorescence microscopy (zonula occludens-1), bile composition (bile salts, phospholipids, lactate dehydrogenase), hepatic gene expression (bile salt transporters and inflammatory mediators) as well as serum and biliary cytokine concentrations were quantified and compared between the study groups. In addition, the integrity of the blood biliary barrier (BBB) was assayed in vivo using horseradish peroxidase (HRP).

\section{Key findings}

LPS administration induced severe small bile duct injury following 6 hours of reperfusion. Furthermore, total bile salts and bilirubin concentrations in serum were increased in the LPS groups compared to sham controls (LPS: +3.3 fold and +1.9 fold, IR + LPS: +3.8 fold and +1.7 fold respectively). The BBB was impaired in the LPS groups as evidenced by elevated levels of HRP in bile ( +4.9 fold), decreased expression of claudin-1 ( -6.7 fold) and claudin-3 (-3.6 fold).

\section{Conclusions}

LPS was found to be a potent inducer of small bile duct injury following hepatic ischemia and 6 hours of reperfusion. This injury was associated with increased permeability of the BBB and impaired hepatic bile salt clearance. 


\section{Introduction}

In the current era of organ shortage, an increasing number of extended criteria donors are considered for transplantation, such as those who donate after cardiac death (DCD). Although short-term graft survival rates similar to that of recipients of organs donated after brain death (DBD) have been reported, the use of these DCD livers has been hampered by the high incidence of ischemic type biliary strictures (ITBS). ${ }^{1}$

Systemic endotoxemia has previously been reported to occur during both organ retrieval and implantation of the liver graft. ${ }^{2,3}$ Furthermore, the underlying cause of death frequently causes increased endotoxin levels in organ donors. ${ }^{4}$ DCD organs in situ are exposed to a period of hypoperfusion and hypoxia prior to the start of cold perfusion All of the intra-abdominal organs become ischemic, including the gut, and this could potentially result in the release of endotoxins into the portal circulation which might then be flushed into the liver during cold perfusion. ${ }^{5}$ Endotoxemia aggravates organ preservation injury and has been associated with increased mortality following transplantation in animal studies. ${ }^{6,7}$ It can substantially exacerbate hepatic ischemia-reperfusion injury (IRI) as it aggravates the inflammatory response by activating liver-resident macrophages and by enhancement of leukocyte recruitment. ${ }^{8}$ If endotoxemia is directly injurious to bile ducts, or augments the effects of ischemia, then potentially it might contribute to ITBS and it would be relevant to determine the acute effect of endotoxin on the biliary tract.

The blood-biliary-barrier (BBB) is formed by tight junctions between cholangiocytes and keeps bile separated from the blood stream. ${ }^{9}$ Several in vitro studies using cultured cholangiocytes have shown that the integrity of these tight junctions is affected by endotoxins, cytokines and ER stress. ${ }^{10,11}$ Moreover, BBB dysfunction has been implicated in the pathophysiology of biliary stricture formation in Mdr2-/- mice, an animal model for primary sclerosing cholangitis (PSC). ${ }^{12}$ In Mdr2-/- mice, tight junction dysfunction was associated with leakage of bile in the portal tract area, with subsequent inflammation, periductal fibrosis and stricture formation. Bile toxicity has already been implicated in the pathophysiology of ITBS and disturbed tight junction architecture was observed in human common bile duct sections following cold preservation and reperfusion. ${ }^{13,14}$ However the relationship between BBB dysfunction and biliary complications was not assessed in this human study.

The aim of the current study was to assess the effect of endotoxins, in the form of lipopolysaccharides (LPS) on the development of biliary injury in a model of warm hepatic-ischemia reperfusion. 


\section{Methods}

\section{Animals}

Male Sprague-Dawley rats weighing between 250-350 grams were obtained from the Animal Resource Centre (ARC, Perth, Australia) and housed in a temperature and light-controlled facility. The Animal Ethics Committee of the University of Queensland (MED/PAH/472/13/PAH) approved the study protocol in accordance with the Australian Code for the Care and Use of Animals for Scientific Purposes.

\section{Surgical procedure and experimental groups}

Sixty-four animals were equally divided over 4 study groups: a sham group (Sham) underwent midline laparotomy only; a LPS group (LPS) underwent midline laparotomy and intra-peritoneal LPS administration; a hepatic ischemia-reperfusion group (IR) underwent 30 minutes of warm hepatic ischemia; and a combined group (IR+LPS) simultaneously received LPS into the peritoneal cavity and warm hepatic ischemia.

Briefly, laparotomy was performed under isoflurane anaesthesia and an atraumatic vascular clamp (BH030R, BBraun, Bethlehem, PA, USA) was placed across the vasculobiliary pedicle of the median and left lateral lobe inducing ischemia to $70 \%$ of the liver avoiding splanchnic congestion (IR groups). Animals also received either vehicle $(0.9 \%$ sterile $\mathrm{NaCl}$ ) or $1 \mathrm{mg} / \mathrm{kg}$ LPS (dissolved in $0.9 \% \mathrm{NaCl}$ ) from Escherichia coli serotype 0111:B4 (L3012, Sigma-Aldrich, St. Louis, MO, USA) into the peritoneal cavity. After thirty minutes of ischemia, the clamp was removed and the abdomen was closed. Administered LPS was not rinsed from the peritoneal cavity prior to closure of the abdomen.

Following 1 or 6 hours of reperfusion, re-laparotomy was performed and the common bile duct cannulated to collect bile. Blood and tissue samples from the liver (ischemic and non-ischemic lobes) and common bile duct were collected for further analysis.

\section{Bile composition analysis}

After establishment of a steady bile flow during 10 minutes, two bile samples were collected. Lactate dehydrogenase (LDH) was measured via colorimetric assay (\#5604-01, Bioo Scientific Corporation, Austin, TX, USA). Phospholipids were measured spectrophotometrically using an enzymatic assay (433-36201, Wako, Osaka, Japan). For quantitative determination of bile salts, a Nexera X2 Ultra High Performance Liquid Chromatography system (Shimadzu, Kyoto, Japan) was used. Further details are provided in the Supplementary Data. The individual bile salt concentrations were used to calculate the hydrophobicity index $(\mathrm{HI}){ }^{15}$ 


\section{Assessment of blood-biliary barrier permeability}

The permeability of the BBB was assessed in vivo using a medium sized protein, viz. horseradish peroxidase (HRP, P8250, Sigma-Aldrich, St. Louis, MO, USA) as previously described by Takakuwa et al. ${ }^{16}$. Sterile water containing $1000 \mathrm{U}$ of HRP was injected in the inferior vena cava 30 minutes after bile duct cannulation. Bile was collected for a further 10 minutes and HRP activity assayed (Amplex red Assay kit, \#A22188, Thermo Fisher Scientific, Waltham, MA, USA).

\section{Biochemical analysis of serum}

Serum alanine transaminase (ALT), aspartate transaminase (AST), $\quad$-glutamyl transferase, alkaline phosphatase and total bilirubin were measured using commercially available kits (Bioo Scientific Corporation, Austin, TX, USA). Total bile salt concentration in serum was measured spectrophotometrically using a $3 \alpha$ hydroxysteroid dehydrogenase enzymatic assay (\#80460, Crystal Chem, Inc., Chicago, IL, USA).

\section{Assessment of small and large bile duct injury}

Hilar and peripheral liver tissue as well as common bile duct were paraffin embedded and $4 \mu \mathrm{m}$ thick sections were cut and stained with hematoxylin and eosin. Small and large intrahepatic bile ducts were identified based on their size (small $\leq 15 \mu \mathrm{m}$

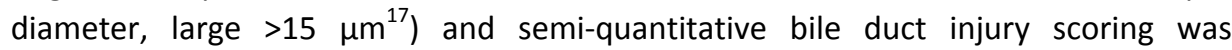
performed. The Small Bile Duct Injury Severity Score (SBDISS) encompassed 2 components: bile duct disruption (adopted from the Banff criteria for acute rejection ${ }^{18}$ ) and ductular proliferation as previously described by Cheng et al.. ${ }^{19}$ Both items were scored using the following grades: 0: absent; 1 : mild; 2 : moderate and 3 : severe. The Large Bile Duct Injury Severity Score (LBDISS) comprised 3 components: ductal disruption and the intra-epithelial and hilar infiltration of inflammatory cells. These components were scored using the same grading from 0-3 as the small bile ducts. Two independent expert pathologists (MG, CC) who were unaware of group assignments performed the scoring.

\section{Immunofluorescent staining of tight junctions}

Hepatic cryosections ( $4 \mu \mathrm{m}$ thick) were incubated with polyclonal rabbit anti-ZO-1 (1:100, 61-7300 Thermo Fisher Scientific, Waltham, MA, USA) and mouse antiCytokeratin 19 (1:100, NCL-CK19, Novocastra) overnight. A donkey anti-rabbit IgG and donkey anti-mouse IgG were used as secondary antibodies (1:2000, A21207 and A21202, Thermo Fisher Scientific, Waltham, MA, USA). DAPI (D9542 Sigma-Aldrich, St. 
Louis, MO, USA) staining was performed using a 1:500 dilution in 5\% donkey serum and $2 \%$ BSA.

\section{RNA extraction and RT-qPCR}

Total RNA was extracted from ischemic and non-ischemic liver lobes and reverse transcribed into complementary DNA as previously described. ${ }^{20}$ Real-time quantitative polymerase chain reaction (RT-qPCR) was performed using the ViiA 7 real-time PCR machine (Invitrogen, Carlsbad, CA, USA) and gene expressions were normalized to the geometric mean expression of 3 reference genes: glyceraldehyde 3-phosphate dehydrogenase, $\beta 2$-microglobulin and basic transcription factor 3 . Primer sequences can be found in Suppl. Table S3.1.

\section{Cytokine array}

The concentrations of Intercellular adhesion molecule-1 (Icam-1), Interferon $\gamma$ (Ifn $\gamma$ ), Interleukin-1ß (II-1ß), Interleukin-10 (II-10), Interleukin-6 (II-6), Leptin, L-Selectin, Monocyte chemoattractant protein-1 (Mcp-1), Tissue inhibitor metalloproteinase-1 (Timp-1) and Tumor necrosis factor- $\alpha$ (Tnf- $\alpha$ ) were assessed using a Quantibody ${ }^{\circledR}$ multiplex ELISA array (QAR-CYT-2; RayBiotech, Norcross, GA, USA). The array was performed according to the manufacturer's instructions on serum (1:2 dilution) and bile (1:4 dilution) samples. The fluorescence intensity was quantified and data were analyzed using the Q-analyzer ${ }^{\circledR}$ (RayBiotech, Norcross, GA, USA).

\section{Statistical analysis}

In each group, eight animals were studied unless stated otherwise. Non-parametric tests were used to evaluate differences between groups, and data are reported as median (inter quartile range). A Kruskal-Wallis test with Dunn's correction for multiple comparisons was performed to compare intervention groups. For categorical data such as histological scoring, a Fisher's exact test was performed. A cut-off $p$-value of 0.05 was used to determine significance. The statistical analysis was performed using GraphPad Prism 7 software (GraphPad, San Diego, CA, USA).

\section{Results}

Ischemia-reperfusion but not LPS induces significant hepatocellular injury

The intra-peritoneal administration of $1 \mathrm{mg} / \mathrm{kg}$ LPS alone had no effect on serum ALT compared to sham operated animals with a median value of 35.5 (31.1-40.3) U/L after 
1 hour and 50.9 (43.7-56.9) U/L after 6 hours of reperfusion, respectively ( $P>0.99$ for both time points) (Figure 3.1). Liver ischemia and reperfusion, however, did induce hepatocellular injury as evidenced by increased serum ALT levels in the IR group at both time points $(1 \mathrm{~h}:+3.1$ fold, $p=0.007 ; 6 \mathrm{~h}:+1.9$ fold, $p=0.046)$. There was no additional effect of LPS on serum ALT in the ischemic group at either time point. This observation was further supported by serum AST elevation in the ischemic groups at the 6-hour time point (Suppl. Table S3.2).

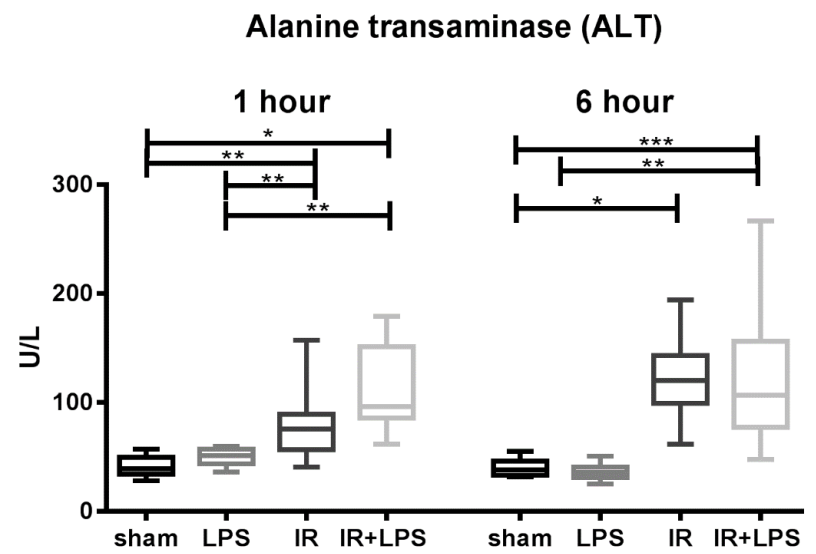

Figure 3.1 Ischaemia-reperfusion results in hepatocellular injury following 1 and 6 hours of reperfusion. Serum alanine transaminase (ALT, U/L) was determined in groups of rats $(n=7-8)$ that were sham-operated, exposed to $1 \mathrm{mg} / \mathrm{kg}$ lipopolysaccharides (LPS) or $70 \%$ hepatic inflow obstruction during 30 minutes (IR), or a combination of the latter two (IR+LPS) followed by reperfusion for 1 and 6 hours. Data are depicted as box and whisker plots showing median, minimum and maximum values. Statistical significance was tested using a Kruskal-Wallis test with Dunn's correction for multiple comparisons, ${ }^{*} p<0.05,{ }^{* *}, p<0.01{ }^{* * *}, p<0.001$.

LPS and not ischemia-reperfusion induces significant small bile duct injury following 6 hours of reperfusion

The intra-hepatic large bile ducts, including the peribiliary plexus, as well as the common bile duct showed no histological signs of injury in any of the study groups (Suppl. Table 3). In contrast, small bile ducts were affected and representative images of each group are shown in Figure 3.2A. Severe small bile duct injury (score $\geq 4$ ) occurred in none of the sham animals and only in a single animal in the IR group (14\%). LPS administration on the other hand caused severe biliary injury in 5 (63\%) animals in both the LPS and IR+LPS group ( $p=0.004)$. In addition to the histological scoring, LDH leakage into bile was measured as a biomarker for biliary injury. ${ }^{21}$ Hepatic IR induced a transient rise in LDH in bile following 1 hour of reperfusion, 
which resolved at the later time point (Suppl. Figure S3.1). Following 6 hours of reperfusion, the administration of LPS, with or without IR, induced a marked increase in biliary LDH compared to sham operated animals (sham: 32.2 (29.5-35.8) U/L, LPS 91.1 (64.3-146.8) U/L, IR+LPS: 147.2 (84.6-234.0) U/L, $p 0.01$ and $p<0.001$ respectively) (Figure 3.2B). Only data from the six-hour time point will be presented below. All results from the one-hour time point can be found in the supplementary data.

A
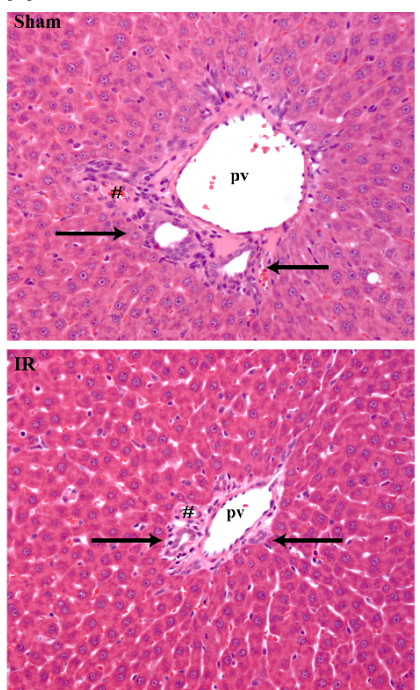

B
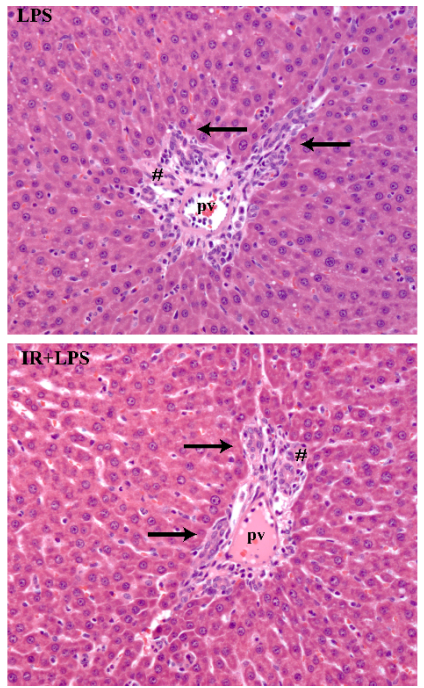

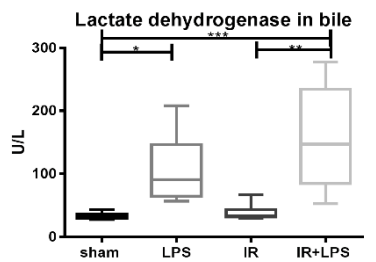

Figure 3.2 Severe small bile duct injury in LPS and IR + LPS groups following 6 hours of reperfusion. Groups of rats ( $n=7-8)$ were sham-operated, exposed to lipopolysaccharides (LPS) or $70 \%$ hepatic inflow obstruction during 30 minutes (IR), or a combination of the latter two (IR+LPS) followed by reperfusion for 6 hours. A) Representative images displaying portal tract histology (magnification 200x) with arrows indicating bile ducts. \#: hepatic artery, PV: portal vein. B) Lactate dehydrogenase (LDH) activity in bile (U/L) depicted as box and whisker plot showing median, minimum and maximum values. Statistical significance was tested using a KruskalWallis test with Dunn's correction for multiple comparisons, ${ }^{*} p<0.05,{ }^{* *} p<0.01,{ }^{* * *} p<0.001$.

\section{LPS impairs handling of biliary constituents following 6 hours of reperfusion}

Bile flow was not significantly different between the intervention groups (Figure 3.3A). LPS administration, with or without IR, did result in a significant increase in the serum bile salt concentration. The mean concentration of total bile salt in serum was 45.7 (33.1-71.2) $\mu \mathrm{mol} / \mathrm{L}$ and $52.0(39.9-61.6) \mu \mathrm{mol} / \mathrm{L}$ in the LPS and IR+LPS group, respectively, compared to $13.7(10.2-16.5) \mu \mathrm{mol} / \mathrm{L}$ in the sham-operated group 
$(p=0.001$, Figure 3.3B). On a transcriptional level, expression of the main bile salt importer $\mathrm{Na}+/$ Taurocholate cotransporting polypeptide (Ntcp) was reduced (Figure 3.3C, $p=0.02$ and $p=0.04$ respectively). Furthermore, LPS administration led to a significant increase in serum bilirubin (figure 3.3D). The bilirubin concentration in the LPS and IR+LPS was 2.1 (1.9-2.5) and 1.9 (1.7-2.4) mg/dL compared to 1.1 (1.0-1.3) and $1.1(1.0-1.5) \mathrm{mg} / \mathrm{dL}$ in the sham and IR groups respectively. This was accompanied by a reduction in mRNA expression of multidrug resistance protein 2 (Mrp2), the ATPbinding cassette protein responsible for secretion of glucuronidated bilirubin into the bile canaliculi, in the LPS group (Suppl. Figure S3.2). Note that, gene expression levels of the non-ischemic lobes will not be discussed in this manuscript but can be found in the Suppl. Figures S3.3 and S3.4.
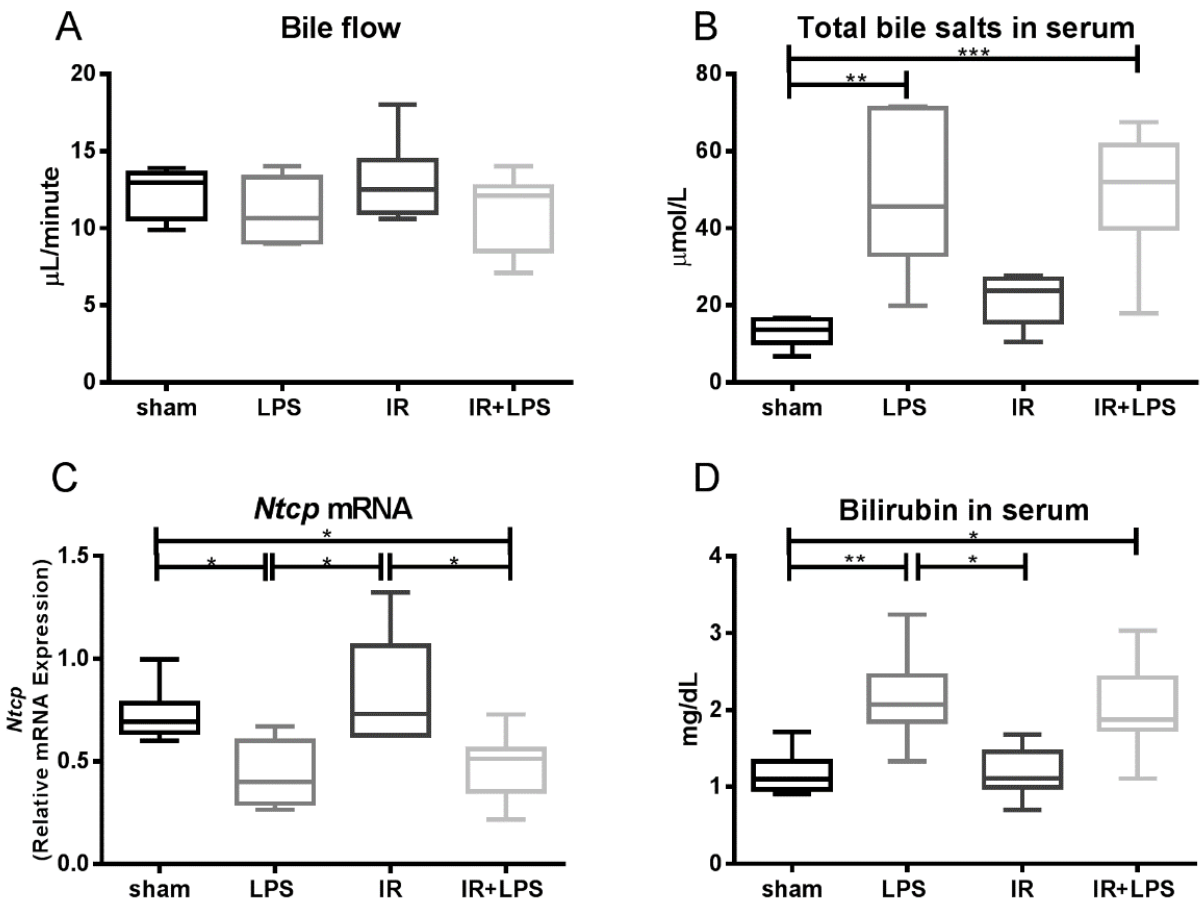

Figure 3.3 Impaired hepatic bile salt clearance following 6 hours of reperfusion. A) Bile flow ( $\mu \mathrm{L} / \mathrm{min})$. B) Concentration of total bile salts in serum ( $\mu \mathrm{mol} / \mathrm{L})$ C) Hepatic Ntcp mRNA expression assessed using real-time quantitative polymerase chain reaction D) Serum bilirubin concentration $(\mathrm{mg} / \mathrm{dL})$. Groups of rats $(\mathrm{n}=7-8)$ were sham-operated, exposed to lipopolysaccharides (LPS) or $70 \%$ hepatic inflow obstruction during 30 minutes (IR), or a combination of the latter two (IR+LPS) followed by reperfusion for 6 hours. Data are depicted as box and whisker plots showing median, minimum and maximum values. Statistical significance was tested using a Kruskal-Wallis test with Dunn's correction for multiple comparisons, $* p<0.05, * * p<0.01$. 
LPS increased the permeability of the blood-biliary-barrier following 6 hours of reperfusion

BBB permeability was measured in vivo using HRP to investigate whether the development of biliary injury in our model was accompanied by BBB dysfunction. IR did not cause an increase in biliary HRP above the background levels observed in the controls, indicating an intact barrier (Figure 3.4A). In the LPS group, however, the BBB was impaired as evidenced by significant leakage of HRP from blood to bile $(+4.9$ fold, $p<0.001)$, with a trend apparent in the IR+LPS group $(+3.7$ fold, $p=0.06)$. At the transcriptional level, the expression of tight junction proteins Claudin- 1 and Claudin- 3 was markedly lower in the LPS and IR+LPS group compared to the sham and IR groups (Figure 3.4B and 3.4C). Conversely, expression of tight junction protein Zonula occludens 1 (Zo-1) was 2-fold and 1.8-fold higher in the LPS and IR+LPS group compared to sham operated animals ( $p<0.01$, Figure 3.4D).

A
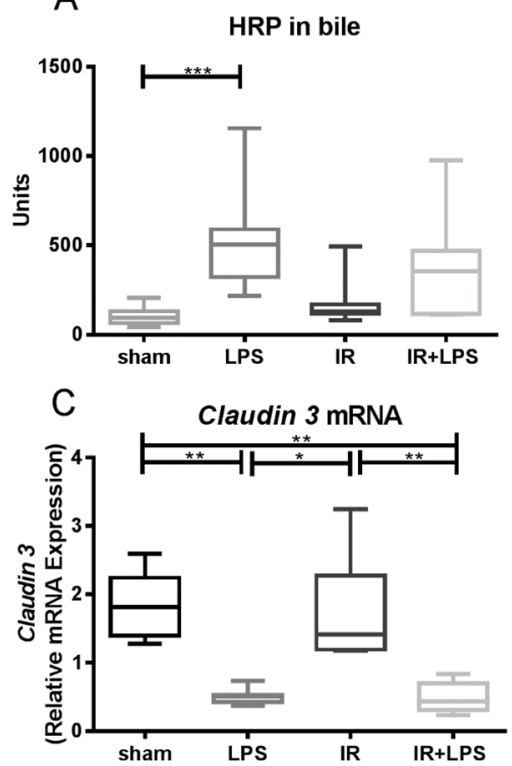

B
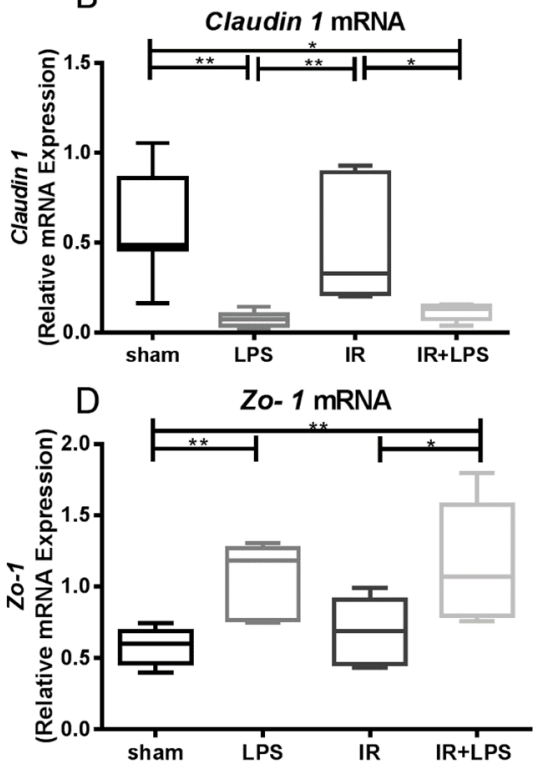

Figure 3.4 Increased permeability of the blood biliary barrier following 6 hours of reperfusion. Groups of rats $(n=7-8)$ were sham-operated, exposed to lipopolysaccharides (LPS) or 70\% hepatic inflow obstruction during 30 minutes (IR), or a combination of the latter two (IR+LPS) followed by reperfusion for 6 hours. Horseradish peroxidase (HRP) output in bile (A) following intravenous injection of 1000IU HRP and hepatic expression of genes encoding tight junction proteins Claudin-1 (B) Claudin 3 (C) and Zo-1 (D) was determined. Data are depicted as box and whisker plots showing median, minimum and maximum values. Statistical significance was tested using a Kruskal-Wallis test with Dunn's correction for multiple comparisons, ${ }^{*} p<0.05,{ }^{* *} p<0.01,{ }^{* * *} p<0.001$ 
Immunofluorescence co-staining for cytokeratin 19 and ZO-1 showed that ZO-1 was present at the junctions of neighbouring cholangiocytes in animals that did not develop biliary injury (Figure 3.5 A-D). Animals that developed biliary injury, such as those in the IR+LPS group, showed markedly altered tight junction morphology with an irregular ZO-1 staining pattern not confined to junctions of neighbouring cholangiocytes (Figure $3.5 \mathrm{E}-\mathrm{H}$ ).

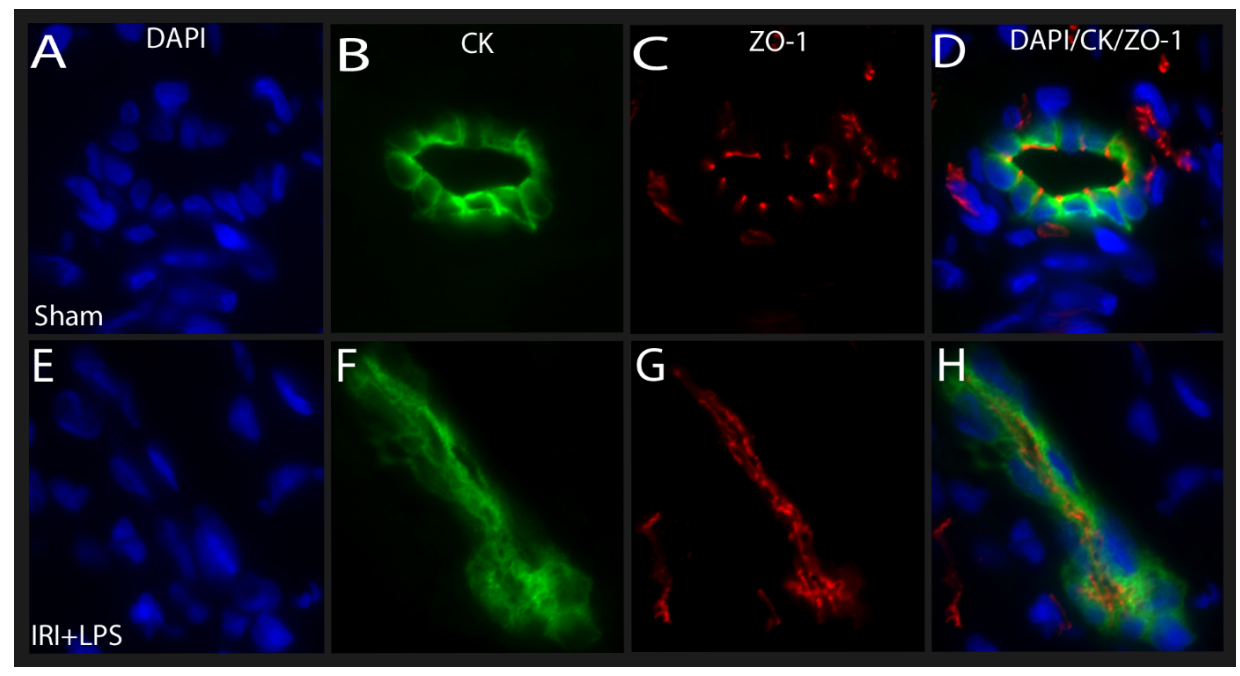

Figure 3.5 Altered tight junction morphology in animals with severe bile duct injury. Immunofluorescence stain for nuclei (blue), cytokeratin (CK) 19 (cholangiocytes; green) and ZO-1 (tight junction protein; red) in representative liver sections from animals without (Sham, group A-D) and with severe biliary injury (IR+LPS group, E-H). Note the irregular pattern of Zo-1 staining that is not confined to neighbouring cholangiocytes in animals exposed to LPS and ischaemia/reperfusion. Images were taken at $60 \mathrm{x}$ magnification.

\section{Bile composition following 6 hours of reperfusion was not toxic}

There were no changes in total bile salt output following LPS administration in the IR+LPS group (Figure 3.6A). Biliary phospholipid output in bile was reduced following LPS administration combined with IR (IR+LPS), compared to IR alone or sham operated animals (sham: 390 (333-490) $\mu \mathrm{g} / 10$ minutes IR: 437 (343-528) $\mu \mathrm{g} / 10$ minutes, IR+LPS: 324.4 (209.6-323.7) $\mu \mathrm{g} / 10$ minutes, $p=0.03$ and $p=0.008$, Figure 3.6B). At the transcriptional level, the expression of the bile salt export pump (Bsep) was not affected in the intervention groups whereas the expression of the canalicular phospholipid flippase (Mdr2) was reduced following LPS administration (Suppl. Figure S3.2). Taken together, the biliary bile salt/phospholipid ratio was unaltered as both 
parameters were affected in a similar fashion (Figure 3.6C). Although the biliary bile salt/phospholipid ratio did not change across the intervention groups, we assessed whether the biliary bile salt pool itself was more hydrophobic and therefore potentially more toxic. Analysis of biliary bile salt composition revealed that glycine and taurine conjugates of cholic acid were most abundant in all groups (Suppl. Table S3.4). The hydrophobicity index (HI) of the biliary bile salt pool was calculated as a surrogate measure of pool toxicity (Figure 3.6D). LPS induced a significant reduction in the $\mathrm{HI}$, suggesting a more hydrophilic and less toxic composition of the biliary bile salt pool $(p=0.02)$.
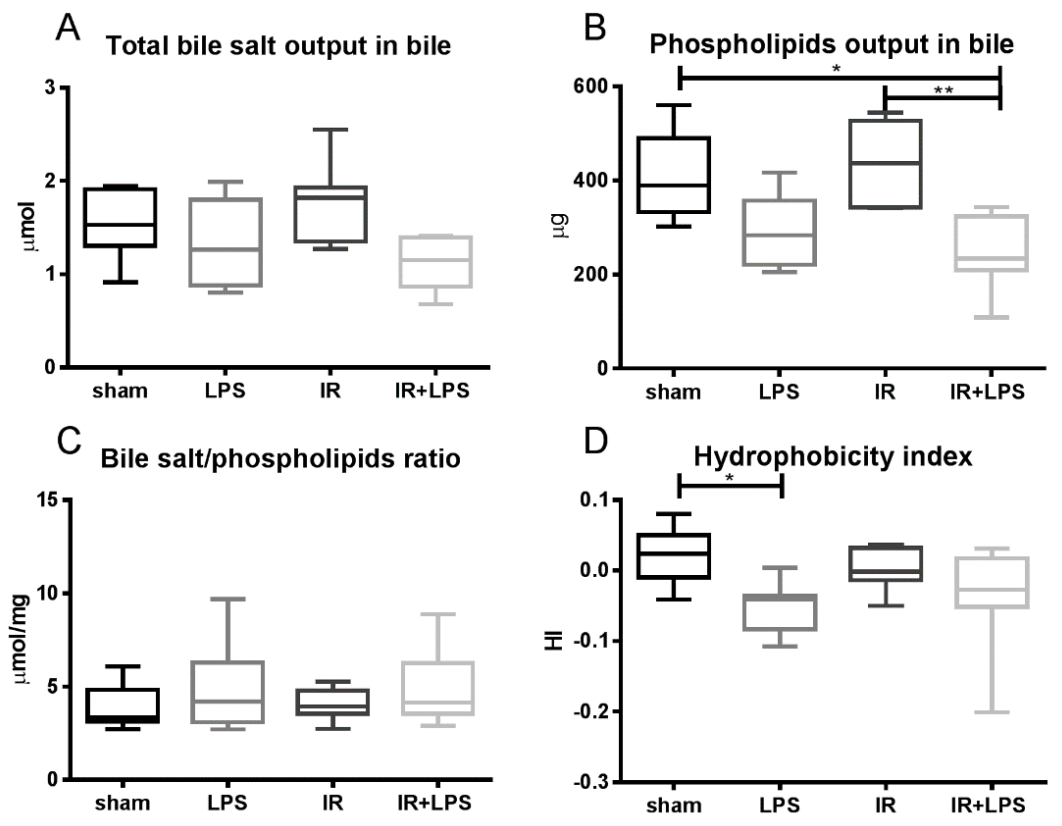

Figure 3.6 Bile composition following 6 hours of reperfusion. A) Total bile salt output ( $\mu$ mol) in bile. B) Phospholipid output $(\mu \mathrm{g})$ in bile C) Ratio of bile salt/phospholipids $(\mu \mathrm{mol} / \mathrm{mg}$ ) D) Hydrophobicity index based on the concentration of individual bile salts. Groups of rats $(n=7-8)$ were sham-operated, exposed to lipopolysaccharides (LPS) or $70 \%$ hepatic inflow obstruction during 30 minutes (IR), or a combination of the latter two (IR+LPS) followed by reperfusion for 6 hours. Data are depicted as box and whisker plots showing median, minimum and maximum values. Statistical significance was tested using a Kruskal-Wallis test with Dunn's correction for multiple comparisons, ${ }^{*} p<0.05,{ }^{* *} p<0.01$

Expression levels of genes coding for key enzymes of both the classical (e.g. Cyp7a1) and alternative (e.g. Cyp7b1) bile salt synthesis pathways were assessed (Figure 3.7). LPS induced a shift from the classical pathway to the alternative (or acidic) bile salt 
synthesis pathway. In the LPS group, gene expression of Cyp7a1 was 5.8 fold lower compared to sham animals ( $p=0.005$, Figure 3.7A) whereas Cyp7b1 and Cyp27a1 expression were 6.4 and 2.6 fold higher, respectively $(p=0.001$ and $p=0.02$, Figure 3.7B and 3.7C). A similar pattern was seen in the IR+LPS groups (Cyp7b1 +10 fold, Cyp27a1 +2.9 fold, $p<0.001$ and $p=0.003$ ). Cyp8b1, coding for sterol $12 \alpha$-hydroxylase, an enzyme determining the hydrophilicity/hydrophobicity balance of newly synthesized bile salts, was significantly lower in the LPS groups (6.7 fold and 7.8 fold, $p=0.02$ and $p=0.003$ Figure 3.7D).
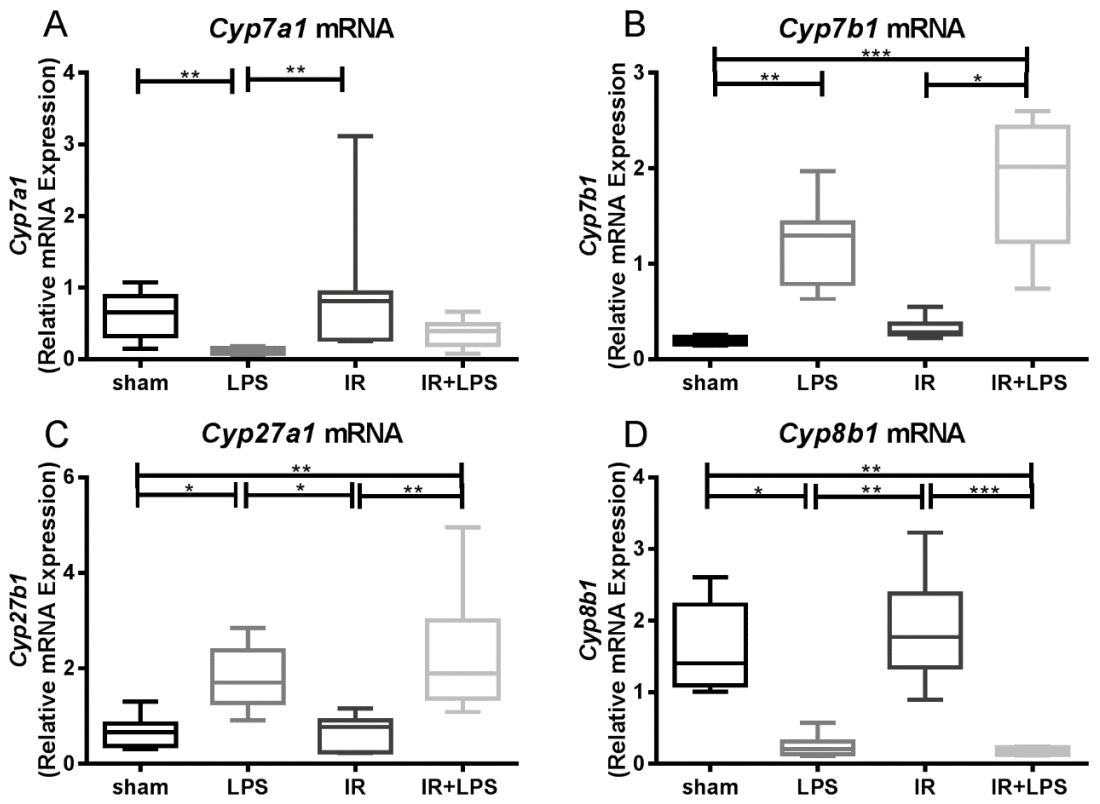

Figure 3.7 Hepatic expression of genes engaged in bile salt synthesis. mRNA levels of Cyp7a1 (A), Cyp7b1 (B), Cyp27a1 (C), and Cyp8b1 (D) were determined by RT-qPCR. Median and left lateral liver segments were assessed in rats ( $n=7-8$ per group) that were sham-operated, exposed to lipopolysaccharides (LPS) or 70\% hepatic inflow obstruction during 30 minutes (IR), or a combination of the latter two (IR+LPS) followed by reperfusion for 6 hours. Data are depicted using box and whisker plots showing median, minimum and maximum values. Statistical significance was tested using a Kruskal-Wallis test with Dunn's correction for multiple comparisons, $* p<0.05, * * p<0.01 * * *, p<0.001$.

\section{Induction of inflammatory response following 6 hours of reperfusion}

To determine the inflammatory response of the liver and bile ducts to LPS administration, cytokines in liver tissue, serum and bile were examined (Figure 3.8, Suppl. Figure S3.5). 


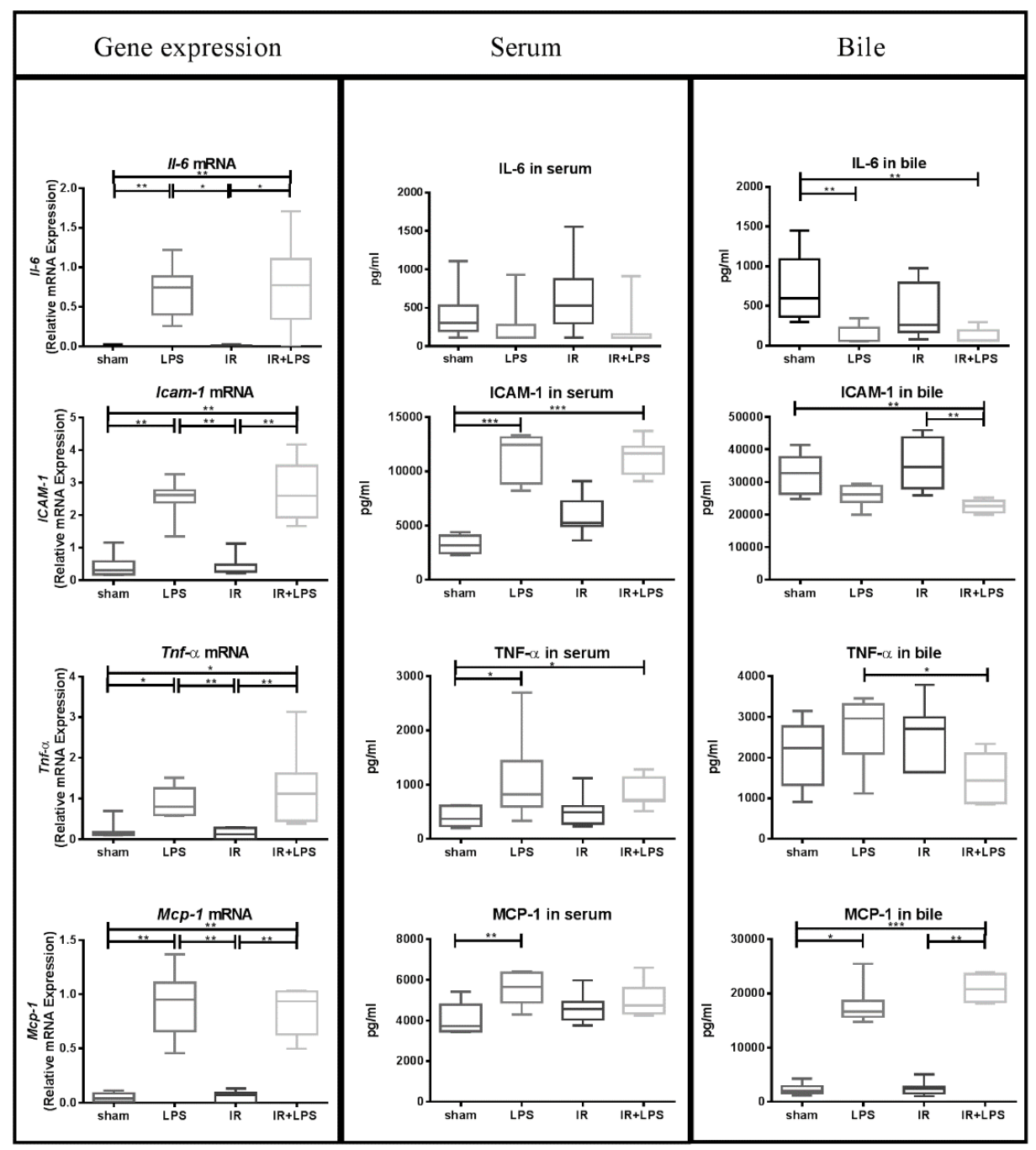

Figure 3.8 Hepatic expression of genes related to inflammation, and cytokine concentrations in serum and bile. Hepatic mRNA expression of II-6, Icam-1, Tnf- $\alpha$, and $M c p-1$ in the median and left lateral liver segments was assessed using real-time quantitative polymerase chain reaction. Cytokine concentrations of II-6, Icam-1, Tnf- $\alpha$ and Mcp-1 in serum and bile (pg/ml). Groups of rats $(n=7-8)$ were sham-operated, exposed to lipopolysaccharides (LPS) or $70 \%$ hepatic inflow obstruction during 30 minutes (IR), or a combination of the latter two (IR+LPS) followed by reperfusion for 6 hours. Data are depicted using box and whisker plots showing median, minimum and maximum values. Statistical significance was tested using a Kruskal-Wallis test with Dunn's correction for multiple comparisons, ${ }^{*} p<0.05, * * p<0.01, * * * p<0.001$. 
Following IP administration of LPS, serum concentrations of Icam-1, Tnf- $\alpha$ and Mcp-1 were significantly increased. Serum II-6 levels were not significantly different between groups. In the liver, IR did not induce an inflammatory response whereas in the LPS groups (LPS and IR+LPS) II-6, Icam-1, Tnf- $\alpha$ and Mcp-1 gene expression was significantly higher compared to the sham and IR groups. As cholangiocytes themselves are capable of producing cytokines such as Mcp-1, II- 6 and Tnf- $\alpha$, their levels in bile were analyzed. Biliary Mcp-1 concentrations were significantly higher in the LPS and IR+LPS groups compared to sham (LPS +8.3 fold, IR+LPS +10.3 fold, $p=0.03$ and $p<0.001$ ). Biliary Icam-1 and II-6 showed an inverse pattern compared to serum and mRNA expression levels with significantly decreased concentrations in the LPS and LPS+IR group. It however needs to be noted that II- 6 levels were overall very low in bile. The concentrations of Timp-1 protein in bile was found to be significantly increased in the LPS groups, matching hepatic gene expression levels (Suppl. Figure S3.5).

\section{Discussion}

The present study demonstrates that endotoxins, in the form of intra-peritoneal administered LPS, are capable of inducing small bile duct injury within 6 hours of exposure in rats. This occurred independently of hepatocellular injury. LPS administration furthermore disturbed the function of the BBB and impaired bile salt clearance in the groups that developed biliary injury.

In the current study, no evidence of biliary injury was found in the IR group following six hours of reperfusion, although increased levels of ALT were observed that time point. This is in conflict with existing literature reporting that cholangiocytes are more vulnerable to reperfusion injury than hepatocytes. ${ }^{22}$ Furthermore, prolonged warm ischemia is a known risk factor for the development of ITBS. ${ }^{13}$ The hepatocellular injury in the current study however might have been transient as the serum half-life of ALT is 4.5 to 8 hours. ${ }^{23}$ This was further supported by the decrease in serum ALT levels between the one and six hour time point. Utilizing a longer duration of ischemia for this experiment may have resulted in biliary injury but the clamp time used was chosen to align with clinical DCD organ procurement. ${ }^{1}$ However, rats respond differently to IR injury than humans as they do not display any evidence of biliary injury on histology following exposure to $\mathbf{3 0}$ minutes of warm and three hours of cold ischemia ${ }^{24}$ and in future experiments, varying clamp times may need to be included.

Since endotoxic shock can be a result of systemic exposure to LPS, it might be argued that the biliary damage seen in the LPS treated groups is the result of ischemic biliary injury due to hypotension (25) although we intended to explore a direct effect of LPS on cholangiocytes. For this reason the dose of endotoxin used in our experiments was 
an order of magnitude lower than the commonly used intra-peritoneal dose of $10-30 \mathrm{mg} / \mathrm{kg}$ that induces shock. ${ }^{25}$ Caraceni et al. have shown previously that $1 \mathrm{mg} / \mathrm{kg}$ LPS alone did not induce a significant drop in systolic blood pressure or liver perfusion. ${ }^{8}$ It was also considered that a lower dose might be more clinically relevant. In the present study, HRP was used to assess the permeability of the BBB in vivo. HRP appears in bile via two distinct routes, trans- and para-cellular, the latter being the fastest across tight junctions (26). More HRP appeared in bile in the LPS groups suggesting a loss of function of the tight junction barrier. This was further supported by almost undetectable expression of Claudin-1 and Claudin-3, two important components of tight junctions. ZO-1 staining showed marked disruption of tight junction morphology with an irregular staining pattern in animals that developed severe biliary injury. Endotoxins were found to disrupt tight junctions in cell culture experiments. ${ }^{10}$ However to the best of our knowledge, the present study is the first report of BBB disruption in an animal model of endotoxemia.

When the BBB is impaired, bile can leak back into the portal area resulting in clinical signs of cholestasis. ${ }^{27}$ Even though bile flow was not significantly impaired in this model, LPS was found to increase the circulating levels of bilirubin and bile salts and to lower the hepatic expression of Ntcp and Mrp2. The impaired tight junction function could be an explanation for elevated serum bile salts. Alternatively, the latter may be due to LPS-mediated repression of bile salt uptake via Ntcp. ${ }^{28}$

This study did not reveal an alteration in biliary bile salt/phospholipid ratio or an increased proportion of hydrophobic/toxic bile acids. This could partially be explained by the use of rats in our experiments since they have less toxic bile compared to humans. ${ }^{15}$ Additionally, our injury model was not extreme, with a short duration of IR, a low dose of LPS and a short follow up duration.

The exact mechanisms by which LPS induces biliary injury in our model remain to be determined. Kupffer cells have previously been shown to play a pivotal role in the development of LPS-enhanced preservation injury and endotoxemia-induced cholestasis, as their depletion from the liver was found to reduce the injury. ${ }^{7,28}$ Cholangiocytes themselves however also participate in the innate and adaptive immune response. They express toll like receptor 4 (TLR-4) and they produce cytokines such as II-6 and Tnf- $\alpha$ in response to LPS. ${ }^{29}$ This TLR mediated immune response not only aids in the defence against pathogens, but it has also been implicated in the pathophysiology of PSC, primary biliary cholangitis and cystic fibrosis-associated cholangiopathies. ${ }^{29-32}$ In the present study, cytokines were detected in bile and their levels were altered by LPS administration. This suggests that the local inflammatory milieu contributes to the development of biliary injury.

Previous studies have linked immunological factors with the development of ITBS, especially when occurring in the periphery of the liver. ${ }^{13,33}$ Furthermore, a study by Friedrich et al. identified that CD14/TLR-4 signaling, a critical element in the innate immune response to factors such as LPS, played a pivotal role in ITBS formation. ${ }^{34}$ In 
the current study we found that, after 6 hours of reperfusion, the biliary Mcp-1 protein concentration was significantly increased upon LPS administration and this chemokine has previously been linked to myofibroblastic transformation and differentiation of portal myofibroblasts ${ }^{35}$ In addition, the concentrations of Timp-1 protein in bile and hepatic gene expression were significantly elevated in the LPS groups, which could suggest the initiation of a fibrotic response. ${ }^{36}$

As with any experimental animal study, though, there are potential design limitations that could affect the interpretation of results and a single set of experiments cannot address all the relevant questions. The difficulty of replicating human ischemic injury conditions in rodents has been alluded to above, as has the choice of endotoxin dose. In the present study only short-term effects were examined and a more complex study will be needed to determine the occurrence of stricture formation over time.

This study has indicated that endotoxins, even at a low concentration of $1 \mathrm{mg} / \mathrm{kg}$, are potent inducers of biliary injury. It might be the case that DCD organ procurement exposes donor livers to higher levels of endotoxins compared to DBD donors due to the period of shock prior to the start of cold perfusion, although human data supporting this is lacking. ${ }^{37}$ Our studies, albeit only using a short duration experimental protocol, indicate that it may be worthwhile exploring endotoxin exposure during human organ retrieval and its effect on the development of ITBS. If endotoxin is implicated in ITBS, then it might be possible to limit endotoxin exposure during organ procurement by ligating or venting the portal vein. 


\section{References}

1. Dubbeld J, Hoekstra H, Farid W, Ringers J, Porte RJ, Metselaar HJ, et al. Similar liver transplantation survival with selected cardiac death donors and brain death donors. Br J Surg. 2010;97(5):744-53.

2. Sun $\mathrm{Y}$, Hardaway RM, Williams $\mathrm{CH}$. Comparison of bacterial translocation during traumatic shock and hemorrhagic shock in rats. Int Surg. 1997;82(2):134-6.

3. Caraceni P, Pertosa AM, Giannone F, Domenicali M, Grattagliano I, Principe A, et al. Antagonism of the cannabinoid CB-1 receptor protects rat liver against ischaemia-reperfusion injury complicated by endotoxaemia. Gut. 2009;58(8):1135-43.

4. Azoulay D, Astarcioglu I, Lemoine A, Dennison A, Mathieu D, Saulnier C, et al. The effects of donor and recipient endotoxaemia on TNF alpha production and mortality in the rat model of syngenic orthotopic liver transplantation. Transplantation. 1995;59(6):825-9.

5. Vajdova K, Smrekova R, Kukan M, Jakubovsky J, van Rooijen N, Horecky J, et al. Endotoxin-induced aggravation of preservation-reperfusion injury of rat liver and its modulation. J Hepatol. 2000;32(1): 112-20.

6. Kojima T, Yamamoto T, Murata M, Chiba H, Kokai Y, Sawada N. Regulation of the blood-biliary barrier: interaction between gap and tight junctions in hepatocytes. Med Electron Microsc. 2003;36(3): 157-64.

7. Sheth $\mathrm{P}$, Delos Santos N, Seth A, LaRusso NF, Rao RK. Lipopolysaccharide disrupts tight junctions in cholangiocyte monolayers by a c-Src-, TLR4-, and LBP-dependent mechanism. Am J Physiol Gastrointest Liver Physiol. 2007;293(1):G308-18.

8. Hanada S, Harada M, Koga H, Kawaguchi T, Taniguchi E, Kumashiro R, et al. Tumor necrosis factoralpha and interferon-gamma directly impair epithelial barrier function in cultured mouse cholangiocytes. Liver Int. 2003;23(1):3-11.

9. Fickert $\mathrm{P}$, Fuchsbichler A, Wagner $\mathrm{M}$, Zollner $\mathrm{G}$, Kaser A, Tilg $\mathrm{H}$, et al. Regurgitation of bile acids from leaky bile ducts causes sclerosing cholangitis in Mdr2 (Abcb4) knockout mice. Gastroenterology. 2004;127(1):261-74.

10. Op den Dries S, Sutton ME, Lisman T, Porte RJ. Protection of bile ducts in liver transplantation: looking beyond ischaemia. Transplantation. 2011;92(4):373-9.

11. Brunner SM, Junger $H$, Ruemmele P, Schnitzbauer AA, Doenecke A, Kirchner GI, et al. Bile duct damage after cold storage of deceased donor livers predicts biliary complications after liver transplantation. J Hepatol. 2013;58(6):1133-9.

12. Heuman DM. Quantitative estimation of the hydrophilic-hydrophobic balance of mixed bile salt solutions. J Lipid Res. 1989;30(5):719-30.

13. Takakuwa $\mathrm{Y}$, Kokai $\mathrm{Y}$, Sasaki K, Chiba H, Tobioka H, Mori M, et al. Bile canalicular barrier function and expression of tight-junctional molecules in rat hepatocytes during common bile duct ligation. Cell Tissue Res. 2002;307(2):181-9.

14. Han Y, Glaser S, Meng F, Francis H, Marzioni M, McDaniel K, et al. Recent advances in the morphological and functional heterogeneity of the biliary epithelium. Exp Biol Med (Maywood). 2013;238(5):549-65.

15. Banff schema for grading liver allograft rejection: an international consensus document. Hepatology. 1997;25(3):658-63.

16. Cheng L, Zhao L, Li D, Liu Z, Chen G, Tian F, et al. Role of cholangiocyte bile Acid transporters in large bile duct injury after rat liver transplantation. Transplantation. 2010;90(2):127-34.

17. Bridle KR, Popa C, Morgan ML, Sobbe AL, Clouston AD, Fletcher LM, et al. Rapamycin inhibits hepatic fibrosis in rats by attenuating multiple profibrogenic pathways. Liver Transpl. 2009;15(10):1315-24.

18. Vajdova K, Smrekova R, Kukan M, Lutterova M, Wsolova L. Bile analysis as a tool for assessing integrity of biliary epithelial cells after cold ischaemia--reperfusion of rat livers. Cryobiology. 2000;41(2):145-52.

19. Noack K, Bronk SF, Kato A, Gores GJ. The greater vulnerability of bile duct cells to reoxygenation injury than to anoxia. Implications for the pathogenesis of biliary strictures after liver transplantation. Transplantation. 1993;56(3):495-500. 
20. The Laboratory Rat. Mark A. Suckow SHW, Craig L. Franklin, editor: Elsevier Inc; 2006.

21. Op den Dries S, Karimian N, Westerkamp AC, Sutton ME, Kuipers M, Wiersema-Buist J, et al. Normothermic machine perfusion reduces bile duct injury and improves biliary epithelial function in rat donor livers. Liver Transpl. 2016;22(7):994-1005.

22. Pillay SP, Wynter C, Lynch S, Wall D, Balderson G, Strong R. Endotoxin levels in donors and recipients during orthotopic liver transplantation. Aust N Z J Surg. 1997;67(4):187-91.

23. Abdala E, Baia CE, Mies S, Massarollo PC, de Paula Cavalheiro N, Baia VR, et al. Bacterial translocation during liver transplantation: a randomised trial comparing conventional with venovenous bypass vs. piggyback methods. Liver Transpl. 2007;13(4):488-96.

24. Pillay SP, Moore TD, Lynch SV, Whiting RF, Balderson GA, Strong RW. Endotoxin levels in adult liver donors. Aust N Z J Surg. 1994;64(9):615-7.

25. Zipfel A, Schenk M, You MS, Lauchart W, Bode C, Viebahn R. Endotoxaemia in organ donors: graft function following liver transplantation. Transpl Int. 2000;13 Suppl 1:S286-7.

26. Rush BF, Jr., Sori AJ, Murphy TF, Smith S, Flanagan JJ, Jr., Machiedo GW. Endotoxaemia and bacteremia during hemorrhagic shock. The link between trauma and sepsis? Ann Surg. 1988;207(5): 549-54.

27. Recknagel P, Gonnert FA, Halilbasic E, Gajda M, Jbeily N, Lupp A, et al. Mechanisms and functional consequences of liver failure substantially differ between endotoxaemia and faecal peritonitis in rats. Liver Int. 2013;33(2):283-93.

28. Lowe PJ, Kan KS, Barnwell SG, Sharma RK, Coleman R. Transcytosis and paracellular movements of horseradish peroxidase across liver parenchymal tissue from blood to bile. Effects of alphanaphthylisothiocyanate and colchicine. Biochem J. 1985;229(2):529-37.

29. Rahner C, Stieger B, Landmann L. Structure-function correlation of tight junctional impairment after intrahepatic and extrahepatic cholestasis in rat liver. Gastroenterology. 1996;110(5):1564-78.

30. Sturm E, Havinga R, Baller JF, Wolters H, van Rooijen N, Kamps JA, et al. Kupffer cell depletion with liposomal clodronate prevents suppression of Ntcp expression in endotoxin-treated rats. J Hepatol. 2005;42(1):102-9.

31. Syal G, Fausther M, Dranoff JA. Advances in cholangiocyte immunobiology. Am J Physiol Gastrointest Liver Physiol. 2012;303(10):G1077-86.

32. Lleo A, Maroni L, Glaser S, Alpini G, Marzioni M. Role of cholangiocytes in primary biliary cirrhosis. Semin Liver Dis. 2014;34(3):273-84.

33. Fiorotto R, Scirpo R, Trauner M, Fabris L, Hoque R, Spirli C, et al. Loss of CFTR affects biliary epithelium innate immunity and causes TLR4-NF-kappaB-mediated inflammatory response in mice. Gastroenterology. 2011;141(4):1498-508, 508.e1-5.

34. Karrar A, Broome U, Sodergren T, Jaksch M, Bergquist A, Bjornstedt $M$, et al. Biliary epithelial cell antibodies link adaptive and innate immune responses in primary sclerosing cholangitis. Gastroenterology. 2007;132(4):1504-14.

35. op den Dries S, Buis Cl, Adelmeijer J, Van der Jagt EJ, Haagsma EB, Lisman T, et al. The combination of primary sclerosing cholangitis and CCR5-Delta32 in recipients is strongly associated with the development of nonanastomotic biliary strictures after liver transplantation. Liver Int. 2011;31(8):1102-9.

36. Friedrich K, Smit M, Brune M, Giese T, Rupp C, Wannhoff A, et al. CD14 is associated with biliary stricture formation. Hepatology. 2016.

37. Kruglov EA, Nathanson RA, Nguyen T, Dranoff JA. Secretion of MCP-1/CCL2 by bile duct epithelia induces myofibroblastic transdifferentiation of portal fibroblasts. Am J Physiol Gastrointest Liver Physiol. 2006;290(4):G765-71.

38. Arthur MJ. Fibrogenesis II. Metalloproteinases and their inhibitors in liver fibrosis. Am J Physiol Gastrointest Liver Physiol. 2000;279(2):G245-9. 


\section{Supplementary material}

Table S3.1 Primer sequences used for real time RT-PCR.

\begin{tabular}{|c|c|c|c|}
\hline Gene & Forward & Reverse & Gene ID \\
\hline Gapdh & tcctgcaccaccaactgcttagc & gcctgcttcaccaccttcttgat & NM_017008.4 \\
\hline Btf3 & ccaaacaatctgtggatgga & tctgccaagtgtcaccttctt & NM_001008309.1 \\
\hline B-2 microglobulin & cctgtgtgcggttttcatc & ttctgccttggagtcctttc & NM_012512.2 \\
\hline Cyp7a1 & caagacgcacctcgctattc & gaggctgctttcattgcttc & NM_012942.2 \\
\hline Cyp7b1 & tcactacagagccgccaga & gcaaggtgaccgcaaaga & NM_0191381 \\
\hline Cyp8b1 & gatccgccgtggagataag & gccatcagggttgaggaa & NM_31241.1 \\
\hline Cyp27a1 & cgatggctgaggaagagaga & agccttgacagcaggagttg & NM_178847.3 \\
\hline Claudin 1 & aagcaccgggcagataca & ccagcaggatgccaattac & NM_031699.2 \\
\hline Claudin 3 & accgcaccatcaccactac & cgtggcgtctgtaaccatc & NM_031700.2 \\
\hline Zo-1 & cctccaaaacaggccattac & ttggtagctgagggcagaac & NM_001106266.1 \\
\hline II-6 & cttccagccagttgccttc & ctggtctgttgtgggtggt & NM_012589.2 \\
\hline Tnf-alpha & agatgtggaactggcagagg & cacgagcgggaatgagaa & X66539.1 \\
\hline II-1 beta & aaaaatgcctcgtgctgtct & tgtcgttgcttgtctctcctt & NM_031512.2 \\
\hline II-10 & aagcaaggcagtggagca & cgggtggttcaatttttca & NM_012854.2 \\
\hline Timp-1 & gccgtttaaggaacggaaa & atggctgaacagggaaacac & NM_053819.1 \\
\hline Icam-1 & gagcgacattggggaaga & cgctctgggaacgaatacac & NM_012967.1 \\
\hline Mcp-1 & gatctgtgctgaccccaataa & cattcaaaggtgctgaagtcc & NM_031530.1 \\
\hline Ntcp & tcaagtccaaaaggccaca & tgcccacattgatgacaga & NM_017047.1 \\
\hline Mdr2 & tcagcaaccagagcagagaa & tgcccaggagcataaacaa & NM_012690.2 \\
\hline Mrp2 & caagcaggtgttcgtcgtgt & acaggaggaactgtggcttg & NM_012833.2 \\
\hline Bsep & gggcagtcacacccatctac & agcccaggatgacaaagaac & NM_031760.1 \\
\hline
\end{tabular}

Table S3.2 Serum liver function tests.

\begin{tabular}{cccccc}
\hline & & Sham & LPS & IR & IR+LPS \\
\hline AST (U/L) & 1 hour & 92.2 & 56.5 & 168.9 & 137.2 \\
& & $(79.9-108.3)$ & $(48.6-75.6)$ & $(110.8-215.2)$ & $(118.1-179.7)$ \\
& 6 hours & 80.9 & 110.3 & 122.6 & 147.9 \\
& & $(69.0-85.7)$ & $(88.0-120.6)$ & $(96.6-123.0)^{\mathrm{a}}$ & $(105.7-189.5)^{\mathrm{a}}$ \\
GGT (U/L) & 1 hour & 2.3 & 2.7 & 2.6 & 2.7 \\
& \multirow{4}{*}{6 hours } & $(1.8-3.1)$ & $(2.6-3.1)$ & $(2.4-3.1)$ & $(2.3-2.9)$ \\
& & 2.2 & 3.8 & 2.5 & 2.8 \\
ALP (U/L) & 1 hour & $(1.6-10.3)$ & $(2.6-5.1)$ & $(1.9-2.7)$ & $(1.6-6.2)$ \\
& & 20.3 & 17.0 & 14.8 & 20.6 \\
& \multirow{2}{*}{6 hours } & $(16.7-24.5)$ & $(16.8-22.1)$ & $(13.2-16.8)$ & $(15.7-22.5)$ \\
& & 16.6 & 18.7 & 19.6 & 21.3 \\
& & $(15.1-20.1)$ & $(17.5-21.6)$ & $(15.3-22.3)$ & $(18.2-24.3)$ \\
\hline
\end{tabular}

Data is shown as median (interquartile range). Groups of rats $(n=7-8$ ) were sham-operated, exposed to lipopolysaccharides (LPS) or $70 \%$ hepatic inflow obstruction during 30 minutes (IR), or a combination of the latter two (IR+LPS) followed by reperfusion for 1 and 6 hours. Statistical significance was tested using a Kruskal-Wallis test with Dunn's correction for multiple comparisons, $\mathrm{P}<0.05$ was considered significant, a: significant versus sham. 
Table S3.3 Large Bile Duct Injury.

\begin{tabular}{|c|c|c|c|c|c|}
\hline Intra-hepatic bile ducts & $\begin{array}{l}\text { Sham } \\
N=7^{*}\end{array}$ & $\begin{array}{l}\text { LPS } \\
\mathrm{N}=8\end{array}$ & $\begin{array}{c}\text { IR } \\
\mathrm{N}=5^{*}\end{array}$ & $\begin{array}{c}\text { IR+LPS } \\
\mathrm{N}=5^{*}\end{array}$ & $p$-value \\
\hline \multicolumn{6}{|l|}{ Epithelial atypia } \\
\hline Score $>2$ & $2(28.6 \%)$ & $5(62.5 \%)$ & $1(20 \%)$ & $4(80 \%)$ & 0.19 \\
\hline \multicolumn{6}{|c|}{$\begin{array}{l}\text { Intra-epithelial infiltration of } \\
\text { inflammatory cells }\end{array}$} \\
\hline Score $>1$ & $1(14.3 \%)$ & $3(37.5 \%)$ & $4(80 \%)$ & $2(40 \%)$ & 0.19 \\
\hline \multicolumn{6}{|c|}{ Hilar infiltration of inflammatory cells } \\
\hline Score $>2$ & $3(42.9 \%)$ & $4(50 \%)$ & $1(20 \%)$ & $1(20 \%)$ & 0.73 \\
\hline \multirow[t]{2}{*}{ Common bile ducts } & Sham & LPS & $\mathrm{IR}$ & IR+LPS & $p$-value \\
\hline & $\mathrm{N}=6^{*}$ & $\mathrm{~N}=5^{*}$ & $\mathrm{~N}=4^{*}$ & $\mathrm{~N}=8$ & \\
\hline \multicolumn{6}{|l|}{ Epithelial atypia } \\
\hline Score $>2$ & $2(33.3 \%)$ & $2(40 \%)$ & $1(25 \%)$ & $3(37.5 \%)$ & 0.99 \\
\hline
\end{tabular}

* From some animals insufficient hilar liver tissue was available to perform the analysis. Groups of rats were sham-operated, exposed to lipopolysaccharides (LPS) or $70 \%$ hepatic inflow obstruction during 30 minutes (IR), or a combination of the latter two (IR+LPS) followed by reperfusion for 6 hours. Data is shown as number (\%). Statistical significance was tested using a Fishers exact test. $P<0.05$ was considered significant.

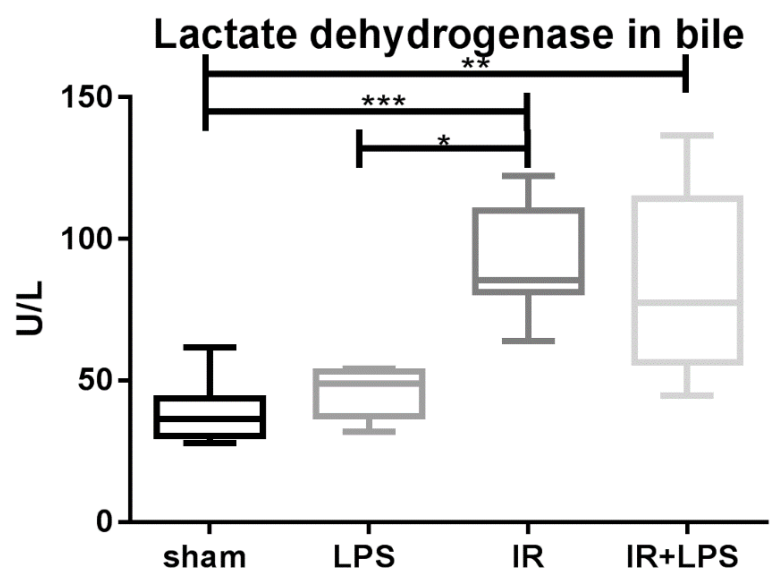

Figure S3.1 Lactate dehydrogenase in bile following 1 hour of reperfusion. Activity of lactate dehydrogenase in bile (U/L) was measured as biomarker for biliary injury. Groups of rats $(n=7-8)$ were sham-operated, exposed to lipopolysaccharides (LPS) or $70 \%$ hepatic inflow obstruction during 30 minutes (IR), or a combination of the latter two (IR+LPS) followed by reperfusion for 1 hour. Data are depicted as box and whisker plots showing median, minimum and maximum values. Statistical significance was tested using a Kruskal-Wallis test with Dunn's correction for multiple comparisons, ${ }^{*} p<0.05,{ }^{* *} p<0.01,{ }^{* * *} p<0.001$. 

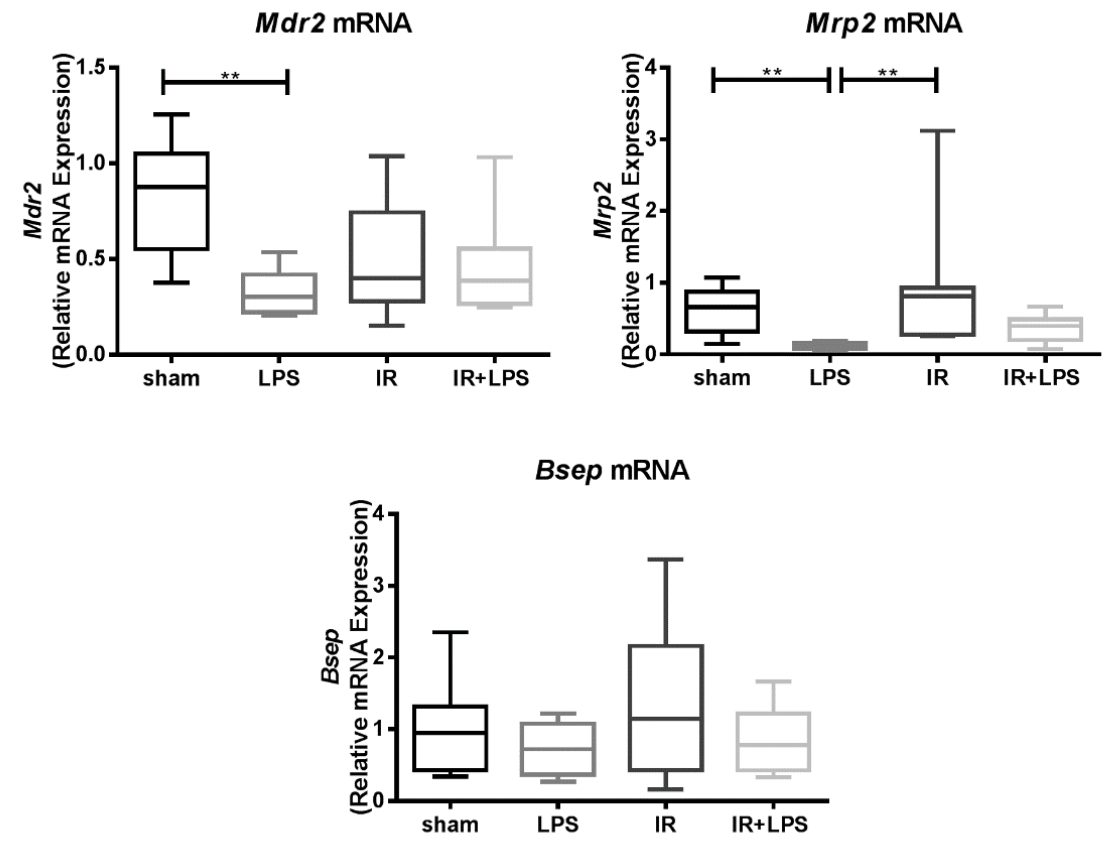

Figure S3.2 mRNA expression of bile salt and organic acid transporters following 6 hours of reperfusion. Hepatic mRNA expression levels of Mdr2, Mrp2 and Bsep in the median and left lateral liver segments following 6 hours of reperfusion assessed using real-time quantitative polymerase chain reaction. Groups of rats $(n=7-8)$ were sham-operated, exposed to lipopolysaccharides (LPS) or $70 \%$ hepatic inflow obstruction during 30 minutes (IR), or a combination of the latter two (IR+LPS) followed by reperfusion for 6 hours. Data are depicted as box and whisker plots showing median, minimum and maximum values. Statistical significance was tested using a Kruskal-Wallis test with Dunn's correction for multiple comparisons, ${ }^{*} p<0.05,{ }^{* *} p<0.01 * * *$, $p<0.001$. 

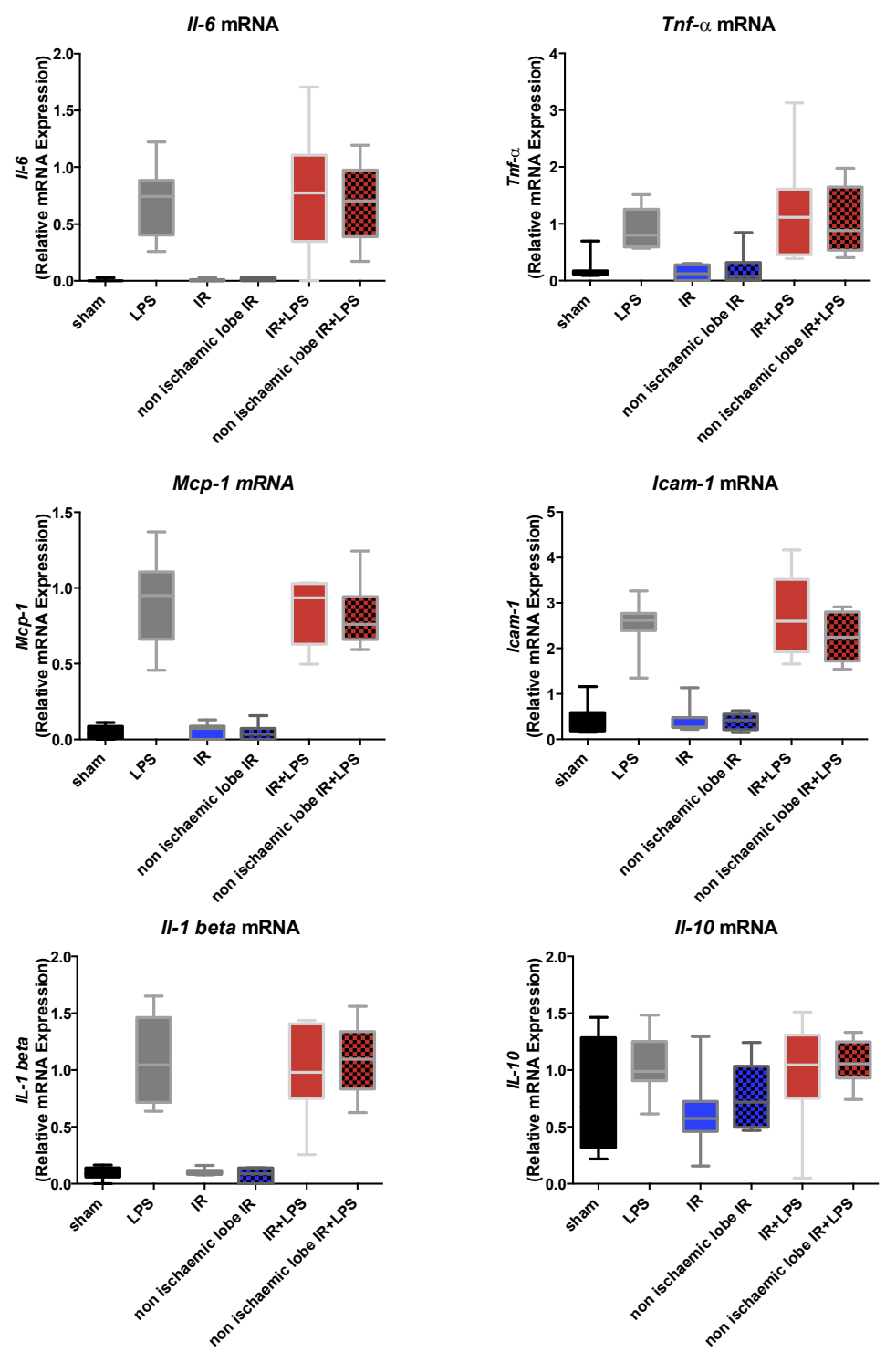

Figure S3.3 Expression of inflammatory genes in the non-ischaemic lobes following 6 hours of reperfusion. Hepatic mRNA expression of $I I-6, \operatorname{Tnf}-\alpha$, Mcp-1, Icam-1, II-1 beta and $I 1-10$ in the median and left lateral liver segments as well as caudate lobe assessed using real-time quantitative polymerase chain reaction. Data are depicted as box and whisker plots showing median, minimum and maximum values. Groups of rats $(n=7-8)$ were sham-operated, exposed to lipopolysaccharides (LPS) or $70 \%$ hepatic inflow obstruction during 30 minutes (IR), or a combination of the latter two (IR+LPS) followed by reperfusion for 6 hours. From the IR and IR+LPS animals, tissue was collected from both the ischaemic and non-ischaemic lobes $(n=6)$. Statistical significance was tested using a Kruskal-Wallis test with Dunn's correction for multiple comparisons comparing Sham with non-ischaemic lobe IR and LPS with nonischaemic lobe IR+LPS, $P<0.05$ was considered significant. 

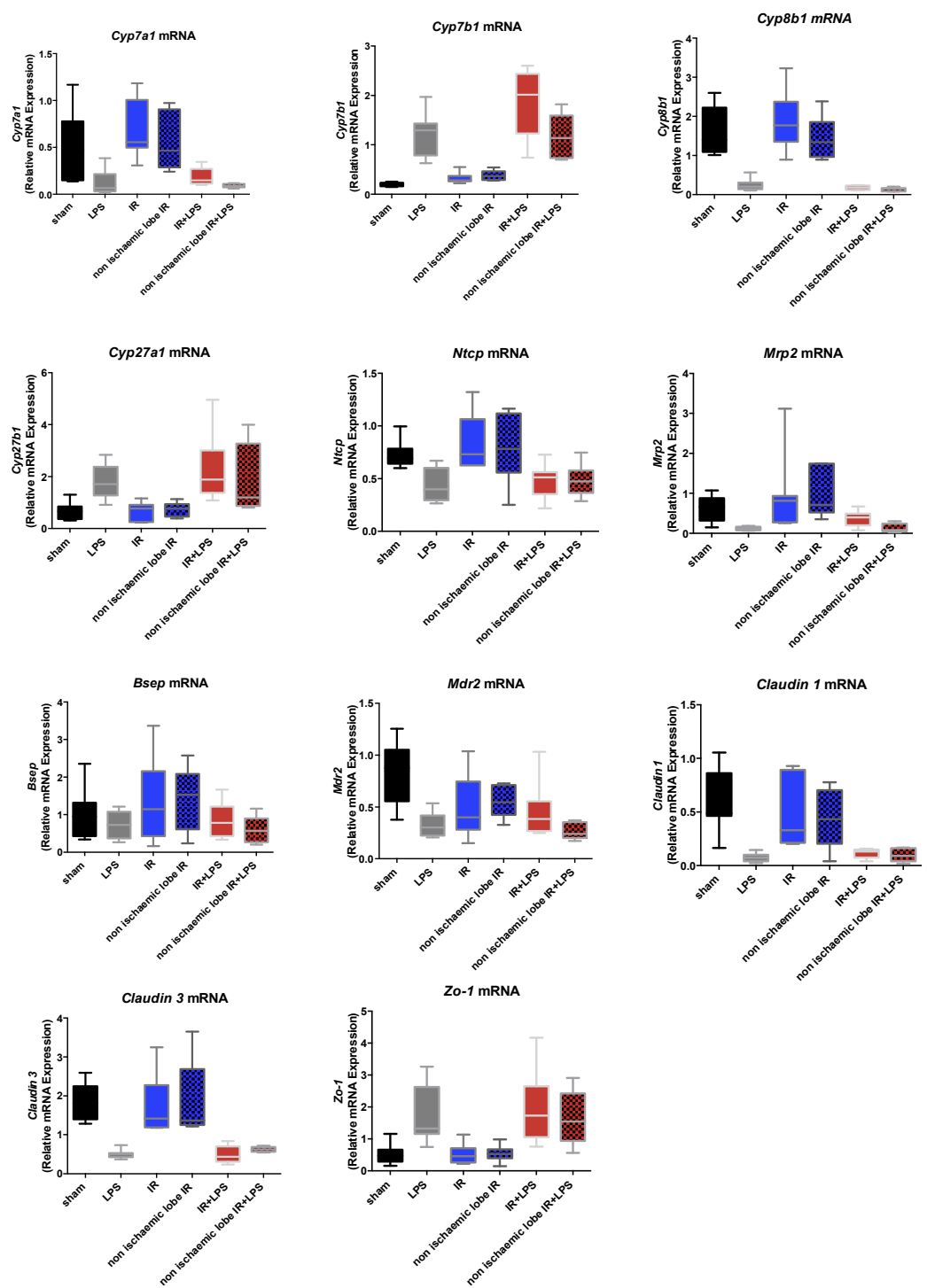

Figure S3.4 Expression of genes engaged in bile salt synthesis and transport, and those encoding for tight junction proteins in the non-ischaemic lobes following $\mathbf{6}$ hours of reperfusion. Hepatic mRNA expression of Cyp7a1, Cyp7b1, Cyp8b1, Cyp27a1, Ntcp, Mrp2, Bsep, Mdr2, Claudin 1, Claudin 3, and Zo-1 in the median and left lateral liver segments as well as caudate lobe assessed using real-time quantitative polymerase chain reaction. Groups of rats $(n=7-8)$ were sham-operated, exposed to lipopolysaccharides (LPS) or $70 \%$ hepatic inflow obstruction during 30 minutes (IR), or a combination of the latter two (IR+LPS) followed by reperfusion for 6 hours. From the IR and IR+LPS animals, tissue was collected from both the ischaemic and non-ischaemic lobes $(n=6)$. Data are depicted as box and whisker plots showing median, minimum and maximum values. Statistical significance was tested using a Kruskal-Wallis test with Dunn's correction for multiple comparisons comparing Sham with non-ischaemic lobe IR and LPS with non-ischaemic lobe IR+LPS, $P<0.05$ was considered significant. 
Table S3.4 Bile salt composition following 6 hours of reperfusion ( $\mu \mathrm{mol} / \mathrm{L})$.

\begin{tabular}{|c|c|c|c|c|}
\hline & Sham & LPS & IR & IR+LPS \\
\hline Ursodeoxycholic acid & N.D. & N.D. & N.D. & N.D. \\
\hline Glycoursodeoxycholic acid & $172(160-232)$ & $171.0(131-270)$ & $160.0(109-176)$ & $111.5(104-171)$ \\
\hline Tauroursodeoxycholic acid & $120(86-181)$ & 95 (88-99) & 117 (107-139) & $64(57-92)$ \\
\hline Cholic acid & $656(493-759)$ & 574 (289-769) & $604.0(332-778)$ & $535.5(418-820)$ \\
\hline Glycocholic acid & 4945 (4168-5380) & $3890(3060-5750)$ & $4310(3320-4920)$ & $3020(2665-3630)^{a}$ \\
\hline Taurocholic acid & $2165(1628-2520)$ & $2390(2200-3350)$ & $3380(2130-3740)$ & $2275(1358-3525)$ \\
\hline Chenodeoxycholic acid & N.D. & N.D. & N.D. & N.D. \\
\hline \multirow[t]{2}{*}{ Glycochenodeoxycholic acid } & $1060(812.8-1373)$ & $816(540-945)$ & $781(611-1140)$ & $485(354-960)^{\mathrm{a}}$ \\
\hline & $535(438-725)$ & $464(355-591)$ & $644(618-740)$ & $322(271-425)^{a, b}$ \\
\hline \multicolumn{5}{|l|}{ Taurochenodeoxycholic acid } \\
\hline Deoxycholic acid & N.D. & N.D. & N.D. & N.D. \\
\hline Glycodeoxycholic acid & $398(269-621)$ & $241.0(148-281)$ & $463(374-514)$ & $229(144-282)$ \\
\hline Taurodeoxycholic acid & $164(118-336)$ & $133(82-218)$ & $295(181-512)$ & $107(89-285)$ \\
\hline Lithocholic acid & N.D. & N.D. & N.D. & N.D. \\
\hline Glycolithocholic acid & N.D. & N.D. & N.D. & N.D. \\
\hline Taurolithocholic acid & N.D. & N.D. & N.D. & N.D. \\
\hline Alpha-Muricholic acid & $102(85-116)$ & 109(83-185) & $98(58-173)$ & $113(81-193)$ \\
\hline Beta-Muricholic acid & N.D. & N.D. & N.D. & N.D. \\
\hline Hyodeoxycholic acid & N.D. & N.D. & N.D. & N.D. \\
\hline Glycohyodeoxycholic acid & $371(170-1010)$ & 307 (231-414) & $533(217-575)$ & $218(141-357)$ \\
\hline Tauro- $\alpha$-Muricholic acid & 745 (543-1021) & $823(665-1020)$ & 989 (850-1090) & $579(501-757)$ \\
\hline Tauro- $\beta$-Muricholic acid & 549 (379-1068) & $862(707-1180)$ & $626(557-902)$ & $728(498-906)$ \\
\hline
\end{tabular}

Data is shown as median (interquartile range). Groups of rats $(n=7-8)$ were sham-operated, exposed to lipopolysaccharides (LPS) or $70 \%$ hepatic inflow obstruction during 30 minutes (IR), or a combination of the latter two (IR+LPS) followed by reperfusion for 1 and 6 hours. Statistical significance was tested using a Kruskal-Wallis test with Dunn's correction for multiple comparisons, $p<0.05$ was considered significant. a: $p<0.05$ compared to sham, b: $p<0.05$ compared to IR, N.D. : not detected. Taurohyodeoxycholic acid was not determined due to a technical issue. 


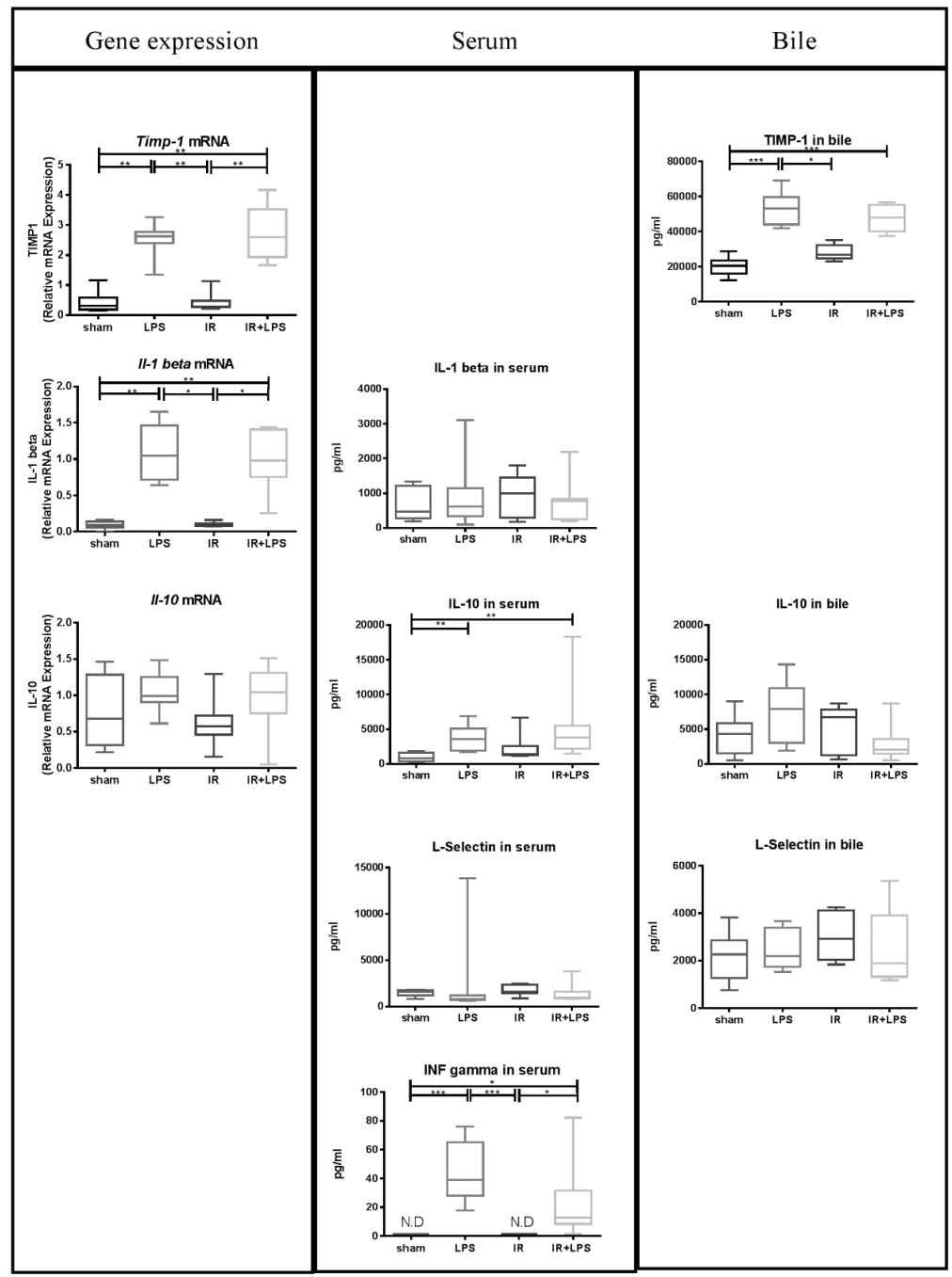

Figure S3.5 Expression of genes related to inflammation, and cytokines concentrations in serum and bile following 6 hours of reperfusion. Hepatic mRNA expression of Timp-1, II-1 beta and II-10 in the median and left lateral liver segments assessed using real-time quantitative polymerase chain reaction. Serum concentrations of II-1 beta, II-10, L-selectin and Ifn- $\nu$ (pg/ml). Biliary concentrations of Timp-1, II-10 and L-selectin (pg/ml). Biliary concentrations of TIMPTimp-1, ILII-10 and L-selectin (pg/ml). Data are depicted as box and whisker plots showing median, minimum and maximum values. Groups of rats $(n=7-8)$ were sham-operated, exposed to lipopolysaccharides (LPS) or $70 \%$ hepatic inflow obstruction during 30 minutes (IR), or a combination of the latter two (IR+LPS) followed by reperfusion for 1 and 6 hours. Statistical significance was tested using a Kruskal-Wallis test with Dunn's correction for multiple comparisons, ${ }^{*} p<0.05,{ }^{* *} p<0.01 * * *, p<0.001$. 


\section{Experimental protocol for the assessment of bile salt composition using an Ultra High Performance Liquid Chromatography system}

\section{Chemicals}

Sigma-Aldrich (St. Louis, MO)

Cholic acid (CA), taurocholic acid (TCA), glycocholic acid (GCA), deoxycholic acid (DCA), taurodeoxycholic acid (TDCA), glycodeoxycholic acid (GDCA), chenodeoxycholic acid (CDCA), taurochenodeoxycholic acid (TCDCA), glycochenodeoxycholic acid (GCDCA), lithocholic acid (LCA), taurolithocholic acid (TLCA), ursodeoxycholic acid (UDCA) and glycoursodeoxycholic acid (GUDCA)

Makaira Ltd (London, England)

Glycolithocholic acid (GLCA)

Merck Millipore (Billerica, MA)

Tauroursodeoxycholic acid (TUDCA)

Steraloids Inc (Newport, RI)

Alpha-muricholic acid ( $\alpha-\mathrm{MCA})$, beta-muricholic acid ( $\beta-\mathrm{MCA})$, omega-muricholic acid

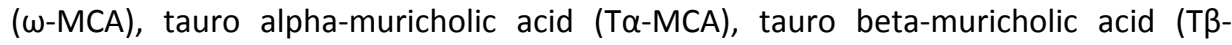
MCA),hyodeoxycholic acid (HDCA), taurohyodeoxycholic acid (THDCA) and glycohyodeoxycholic acid (GHDCA)

CDN Isotopes (Pointe-Claire, Quebec, Canada)

D4-cholic acid (D4-CA), D4-chenodeoxycholic acid (D4-CDCA), D4glycochenodeoxycholic acid (D4-GCDCA) and D4-glycocholic acid (D4-GCA)

Medical Isotopes (Pelham, NH)

D4-taurochenodeoxycholic acid (D4-TCDCA) and D4-taurocholic acid (D4-TCA)

\section{Sample preparation}

Bile samples were diluted in ultrapure water (1:1000) and subsequently homogenized. One quality control standard plasma sample was included for every 10 tested samples. $25 \mu \mathrm{L}$ diluted bile was transferred into a clean tube and $250 \mu \mathrm{L}$ of internal standard solution was added. The sample was vortexed for $60 \mathrm{~s}$ and centrifuged at 
$15800 \times \mathrm{g}$ for 10 minutes. The supernatant was then poured into a clean glass tube and the fluid was evaporated under nitrogen at $40^{\circ} \mathrm{C}$. If samples were not measured immediately, they were stored in this stage at $-20^{\circ} \mathrm{C}$.

Before measurement, samples were reconstituted in $200 \mu \mathrm{L} 50 \%$ methanol in water, vortexed for $60 \mathrm{~s}$ and centrifuged for $3 \mathrm{~min}$ at $1800 \mathrm{x}$ g. The supernatant was subsequently transferred into a $0.2 \mu \mathrm{m}$ spin-filter and centrifuged at $2000 \mathrm{x} \mathrm{g}$ for $10 \mathrm{~min}$. After filtering, the samples were transferred into LC-MS vials and analysed (10 $\mu \mathrm{L}$ injection volume).

\section{Instrumentation}

For the quantitative determination of bile acids we used a Nexera X2 Ultra High Performance Liquid Chromatography system (SHIMADZU, Kyoto, Japan), coupled to a SCIEX QTRAP 4500 MD triple quadruple mass spectrometer (SCIEX, Framingham, MA, USA) (UHPLC-MS/MS).

The LC-MS/MS system is controlled by Analyst MD 1.6.2 software.

\section{Liquid chromatographic and mass spectrometric conditions:}

Bile acids were separated with an ACQUITY UPLC BEH C18 Column (1.7 $\mu \mathrm{m} \times 2.1$ x 100 $\mathrm{mm})$ equipped with an ACQUITY UPLC BEH C18 VanGuard Pre-Column $(1.7 \mu \mathrm{m} \times 2.1 \mathrm{x}$ $5 \mathrm{~mm}$ ), (Waters, Milford, MA, USA). Separation was achieved in 28 minutes using $10 \mathrm{mM}$ ammonium acetate in $20 \%$ acetonitrile (mobile phase $\mathrm{A}$ ) and $10 \mathrm{mM}$ ammonium acetate in $80 \%$ acetonitrile (mobile phase $B$ ), total flow rate: $0.4 \mathrm{ml} / \mathrm{min}$.

The mass spectrometer (MS) parameters such as temperature, ion spray voltage, gas pressures, etc., were optimised by infusing the bile acids and internal standards (IS) in a $50 \% \mathrm{MeOH}$ solution via the internal syringe pump of the mass spectrometer.

Bile acids with the same molar mass were infused as a mixture.

All bile acids and internal standards were detected in negative mode with the mass spectrometer settings shown in Table S3.6.

The multiple reaction monitoring (MRM) transitions for each bile acid and internal standard, as well as their optimum MS parameters such as collision energy (CE), declustering potential (DP), entrance potential (EP) and collision cell exit potential (CXP) are shown in Table S3.7. 
Table S3.6 Mass spectrometer source settings API4500.

\begin{tabular}{ll}
\hline Detection mode & MRM, negative mode \\
Resolution Q1 & Unit (scan speed 10 Da/s) \\
Resolution Q3 & Unit (scan speed 10 Da/s) \\
lonSpray Voltage (IS) & -4500 \\
Temperature & $500^{\circ} \mathrm{C}$ \\
Curtain gas (CUR) & 40 \\
CAD gas (CAD) & -3 \\
Gas 1 (GS1) & 40 \\
Gas 2 (GS2) & 70 \\
\hline
\end{tabular}

Table S3.7 MRM settings API4500.

\begin{tabular}{lccccccc}
\hline Component & $\begin{array}{c}\text { Q1 } \\
\text { mass } \\
\text { (Da) }\end{array}$ & $\begin{array}{c}\text { Q3 mass } \\
\text { (Da) }\end{array}$ & $\begin{array}{c}\text { Declustering } \\
\text { potential } \\
\text { (DP) }\end{array}$ & $\begin{array}{c}\text { Collions } \\
\text { energy } \\
\text { (CE) }\end{array}$ & $\begin{array}{c}\text { Entrance } \\
\text { potential } \\
\text { (EP) }\end{array}$ & $\begin{array}{c}\text { Collision } \\
\text { cell exit } \\
\text { potential } \\
\text { (CXP) }\end{array}$ & $\begin{array}{c}\text { Dwell } \\
\text { (msec) }\end{array}$ \\
\hline UDCA, HDCA, CDCA, DCA & 391.3 & 391.3 & -140.0 & -12.0 & -8.0 & -10.0 & 50.0 \\
CA, MCA's & 407.3 & 407.3 & -120.0 & -12.0 & -8.0 & -10.0 & 50.0 \\
GUDCA, GHDCA, GCDCA, GDCA & 448.4 & 74.0 & -120.0 & -80.0 & -8.0 & -10.0 & 50.0 \\
GCA & 464.2 & 74.0 & -120.0 & -85.0 & -8.0 & -10.0 & 50.0 \\
TUDCA, THDCA, TCDCA, TDCA & 498.3 & 80.0 & -120.0 & -110.0 & -8.0 & -10.0 & 50.0 \\
TCA, T-MCA'S & 514.3 & 79.9 & -120.0 & -115.0 & -8.0 & -10.0 & 50.0 \\
LCA & 375.2 & 375.2 & -120.0 & -25.0 & -8.0 & -10.0 & 50.0 \\
GLCA & 432.2 & 74.0 & -120.0 & -75.0 & -8.0 & -10.0 & 50.0 \\
TLCA & 482.2 & 79.9 & -120.0 & -110.0 & -8.0 & -10.0 & 50.0 \\
D4-CA & 411.3 & 411.3 & -120.0 & -12.0 & -8.0 & -10.0 & 50.0 \\
D4-GCA & 468.2 & 74.0 & -120.0 & -85.0 & -8.0 & -10.0 & 50.0 \\
D4-TCA & 518.3 & 79.9 & -120.0 & -115.0 & -8.0 & -10.0 & 50.0 \\
D4-GCDCA & 452.4 & 74.0 & -120.0 & -80.0 & -8.0 & -10.0 & 50.0 \\
D4-TCDCA & 502.3 & 80.0 & -120.0 & -110.0 & -8.0 & -10.0 & 50.0 \\
D4-CDCA & 395.3 & 395.3 & -120.0 & -12.0 & -8.0 & -10.0 & 50.0 \\
\hline
\end{tabular}




\section{Chapter 4}

\section{The role of macrophages in the development of \\ biliary injury in a lipopolysaccharide enhanced \\ hepatic ischaemia-reperfusion model}

J Reiling

KR Bridle

FG Schaap

L Jaskowski

N Santrampurwala

L Britton

CM Campbell

SWM Olde Damink

DHG Crawford

CHC Dejong

J Fawcett

Submitted 


\section{Abstract}

\section{Background}

Endotoxins, in the form of lipopolysaccharides (LPS), are potent inducers of biliary injury. However the mechanism by which this develops remains unclear. We hypothesised that macrophages are pivotal in the development of LPS-induced biliary injury and no injury would occur in their absence.

\section{Method}

Clodronate liposomes were used to deplete macrophages from the liver. Forty-eight rats were equally divided across six study groups: sham operation (sham), liposome treatment and sham operation (liposomes+sham), $1 \mathrm{mg} / \mathrm{kg}$ LPS i.p. (LPS), liposome treatment and LPS administration (liposomes+LPS), hepatic ischaemia-reperfusion injury with LPS administration (IRI+LPS) and liposome treatment followed by IRI+LPS (liposomes+|RI+LPS). Following six hours of reperfusion, blood, bile, and liver tissue was collected for further analysis. Small bile duct injury was assessed, serum liver tests were performed and bile composition was evaluated. The permeability of the blood biliary barrier (BBB) was assessed using horseradish peroxidase (HRP).

\section{Key findings}

The presence of hepatic macrophages was reduced by $90 \%$ in the liposome pre-treated LPS and $\mid \mathrm{RI}+\mathrm{LPS}$ groups $(P<0.001)$. Despite macrophage depletion, severe small bile duct injury occurred in $4(50 \%)$ animals of the liposomes+IRI+LPS group compared to $6(75 \%)$ animals of the liposomes+LPS group. Furthermore, BBB impairment persisted with leakage of HRP in bile. LPSinduced elevation of the chemokine Mcp-1 in bile was unaffected by macrophage depletion.

\section{Conclusions}

The depletion of macrophages did not prevent the development of biliary injury following LPS or LPS-enhanced IRI. Cholangiocyte rather than macrophage activation may underlie this injury. 


\section{Introduction}

Ischaemic-type biliary stricture (ITBS) formation remains one of the most troublesome complications following liver transplantation using livers donated after circulatory death (DCD). ${ }^{1,2}$ During the DCD retrieval process, ischaemia of intra-abdominal organs such as the gut occurs, and this may result in the release of endotoxins into the portal circulation. ${ }^{3}$ We previously showed that endotoxins, in the form of lipopolysaccharides (LPS), are potent inducers of biliary injury. ${ }^{4}$ Administration of LPS in a hepatic ischaemia-reperfusion injury (IRI) model resulted in severe small bile duct injury, which was characterised by degenerative changes of cholangiocytes and ductular proliferation. The injury was associated with the development of the clinical features of cholestasis and increased leakage of macromolecules across the bloodbiliary-barrier (BBB). ${ }^{4}$ In the present study, we further explored the mechanisms by which LPS induced biliary injury in this model by addressing the involvement of liverresident macrophages.

Kupffer cells respond to LPS via activation of the Toll-like receptor-4 (TLR-4) pathway. ${ }^{5}$ Kupffer cell activation plays a pivotal role in the development of IRI. ${ }^{6}$ Upon activation by endogenous damage-associated and/or pathogen-associated molecular pattern (DAMP/PAMP) molecules, Kupffer cells produce reactive oxygen species and proinflammatory cytokines, which result in the recruitment of neutrophils and the development of tissue damage. ${ }^{7}$ Through enhanced Kupffer cell activation, endotoxaemia can substantially aggravate IRI, as was observed during organ retrieval and liver transplantation. ${ }^{7-10}$ Moreover, primary graft non-function following DCD liver transplantation was associated with a higher degree of Kupffer cell activation. ${ }^{11}$ In animal models, IRI and LPS-enhanced IRI was ameliorated by depleting Kupffer cells as evidenced by reduced hepatocellular damage, increased portal flow, and decreased recipient mortality. ${ }^{8,12}$ It is currently unclear whether macrophages have a role in the development of LPS-induced biliary injury.

The role of cholangiocytes in immune responses is increasingly recognised. ${ }^{13}$ They express various TLRs, and upon stimulation they release pro-inflammatory cytokines such as tumor necrosis factor- $\alpha$ (Tnf- $\alpha$ ) and interleukin- 6 (II-6) in the bloodstream and biliary compartment. ${ }^{13,14}$ Additionally, interferon- $\gamma$ (IFN $\gamma$ ) and Tnf- $\alpha$ increase the expression of T/r4 on cholangiocytes in vitro augmenting their response to LPS. ${ }^{15}$ Lastly, cholangiocytes can act as antigen-presenting cells as they express major histocompatibility complex II (MHC II) on their surface. ${ }^{16}$ Collectively, these data suggest that LPS-induced damage of the cholangiocytes might be a direct effect or mediated via Kupffer cell response.

The aim of the current study was to explore the role of macrophages in the development of biliary injury resulting from LPS administration in a hepatic IRI model. 
We hypothesised that in the absence of macrophages, the BBB would remain intact and that biliary injury would be ameliorated.

\section{Methods}

\section{Animals and experimental groups}

Male Sprague-Dawley rats (250 g; Animal Recourses Centre, Perth, Australia) were used for this study. Procedures were in accordance with the Australian Code for the Care and Use of Animals for Scientific Purposes and approved by the Animal Ethics Committee of the University of Queensland.

For the purpose of the study, forty-eight animals were equally divided across six study groups:

(1) laparotomy without any additional intervention (sham); (2) administration of 1 $\mathrm{mg} / \mathrm{kg}$ LPS in the peritoneal cavity (LPS); (3) 30 minutes of liver ischaemia and simultaneous administration of LPS in the peritoneal cavity (IRI+LPS); (4) pretreatment with clodronate liposomes followed by laparotomy (liposomes+sham); (5) pre-treatment with clodronate liposomes followed by administration of LPS in the peritoneal cavity (liposomes+LPS) and (6) pre-treatment with clodronate liposomes followed by 30 minutes of liver ischaemia and simultaneous administration of LPS in the peritoneal cavity (liposomes+IRI+LPS).

\section{Macrophage depletion and surgical procedure}

Clodronate encapsulated in liposomes $(5 \mathrm{mg}$ clodronate per millilitre, www.clodronateliposomes.org, Amsterdam, the Netherlands) was used to deplete macrophages. ${ }^{17}$ Animals in groups 4, 5 and 6 were injected with clodronate liposomes ( $0.5 \mathrm{ml} / 100$ grams body weight) in the tail vein 48 hours prior to surgical intervention. No PBS or saline liposomes were administered to animals in group 1,2 and 3 as they might disable the phagocytosis function of macrophages by saturation. ${ }^{18}$

Midline laparotomy was performed under general anaesthesia with isoflurane and the common bile duct, hepatic artery and portal vein of the left lateral and medial lobes were identified. A vascular clamp (BH030R, BBraun, Bethlehem, PA, USA) was then placed across these structures to induce ischaemia to approximately $70 \%$ of the liver (IRI groups). Simultaneously, vehicle (0.9\% sterile saline) or $1 \mathrm{mg} / \mathrm{kg}$ LPS from Escherichia coli serotype 0111:B4 (L3012, Sigma-Aldrich, St. Louis, MO, USA; in sterile saline) was administered in the peritoneal cavity. Following 30 minutes of ischaemia, the clamp was removed and the animals were allowed to recover.

Following six hours of reperfusion, re-laparotomy was performed and a cannula was inserted in the common bile duct to allow for bile collection. Animals were 
subsequently euthanised by exsanguination, and liver tissue from the left lateral and median lobes (subjected to ischaemia in the IRI groups) was collected for RNA extraction and (immuno) histological evaluation.

\section{Immunohistochemical staining for mature Kupffer cells}

Paraffin-embedded liver sections stained with a monoclonal antibody against CD163 (ED-2, 1:300, Serotec, Oxford, UK) were used to visualise the presence of mature macrophages. The average number of positive cells per field were counted in five nonoverlapping fields at $200 \times$ magnification using ImageJ image analysis software. ${ }^{19}$

\section{Serum and bile analysis}

Serum alanine transaminase (ALT), aspartate transaminase (AST), $\quad$-glutamyl transferase (GGT), alkaline phosphatase (ALP), and bilirubin, as well as lactate dehydrogenase (LDH) in bile, were assessed using commercially available kits (Bioo Scientific Corporation, Austin, TX, USA). Total bile salt concentration in serum and bile, as well as phospholipid concentration in bile, were measured enzymatically using a kit according to the manufacturer's instructions (\#80460, Crystal Chem, Inc., Chicago, IL, USA and \#433-36201, Wako, Osaka, Japan).

\section{Blood-biliary-barrier integrity assessment}

Horseradish peroxidase (HRP, Peroxidase from horseradish type II, P8250, SigmaAldrich, St. Louis, MO, USA) was used to assess the permeability of the BBB in vivo as previously described. ${ }^{20}$ Two hundred microliters of HRP (5000 IU/mL in sterile water), was injected in the inferior vena cava of each animal. Bile was subsequently collected for 10 minutes to assess para-cellular transport across tight junctions. ${ }^{21} A$ commercially available kit (\#A22188, Thermo Fisher Scientific, Waltham, MA, USA) was used to determine activity of HRP in bile.

\section{RNA extraction and real-time polymerase chain reaction}

Total RNA was extracted from liver tissue using Trisure and CDNA was synthesised using a kit (SensiFAST, Bioline, Taunton, MA, USA). Real-time quantitative polymerase chain reaction (RT-qPCR) was performed on a Viia7 Real-time PCR system (Invitrogen, Carlsbad, CA, USA) using Sybr Green chemistry (Bioline, Taunton, MA, USA). Normalisation was performed using the geometric mean of glyceraldehyde phosphate dehydrogenase, $\beta_{2}$-microglobulin and basic transcription factor 3 mRNA expression. The primer nucleotide sequences and accession numbers can be found in Supplementary Table S4.1. 


\section{Histological evaluation}

Paraffin-embedded liver sections, stained with haematoxylin and eosin, were used for the semi-quantitative assessment of small bile duct injury. Depending on the size of the ducts, ${ }^{22}$ a small bile duct injury severity score (SBDISS) was used as previously described by Cheng et al.. ${ }^{23}$ The score was based on bile duct damage (adapted from the Banff criteria for acute rejection) and ductular proliferation, each of which scored from 0 (absent) to 3 (severe). An expert liver pathologist (CC) who was blinded to study group allocation performed the histological scoring.

\section{Cytokine array on bile samples}

Biliary concentrations of cytokines were determined to gain insight into the local inflammatory milieu surrounding cholangiocytes. Levels of intercellular adhesion molecule-1 (Icam-1), monocyte chemoattractant protein-1 (Mcp-1), tissue inhibitor metalloproteinase-1 (Timp-1), II-6, interleukin-10 (II-10) and L-selectin concentrations were determined using a multiplex ELISA array (QAR-CYT-2, RayBiotech, Norcross, GA, USA). Diluted bile samples were analysed according to the manufacturer's instructions. Fluorescence intensity was determined and data analysis was performed using the Q-analyser (RayBiotech, Norcross, GA, USA).

\section{Statistical analysis}

GraphPad Prism 7 software (GraphPad, San Diego, CA, USA) was used for statistical analysis. Continuous variables are reported as median [interquartile range] and graphed using box plots. A Kruskal-Wallis test was performed to compare all experimental groups, and in case of significant outcome was followed by three predefined post-hoc comparisons with Dunn's correction for multiple comparison to evaluate the effect of macrophage depletion: sham versus liposomes+sham, LPS versus liposomes+LPS and IRI+LPS versus liposomes+IRI+LPS. Categorical variables are reported as frequencies (\%) and represented in tabular form. A Fisher's exact test was performed to compare categorical data. Eight animals were studied per group unless stated otherwise and a $p$-value $<0.05$ was considered statistically significant.

\section{Results}

\section{Effective depletion of Kupffer cells from the liver}

Effectiveness of clodronate to deplete Kupffer cells was studied by CD163 immunohistochemistry (Figure 4.1). In the sham group there were 105 [90-128] $\mathrm{CD} 163^{+}$cells per field. In the LPS and IRI+LPS groups this increased to 158 [147-176] 
and 145 [130-170] per field. Following liposome pretreatment, the number of positive cells per field decreased with $84 \%(p=0.01), 90 \% p<0.001$ and $89 \%(p<0.001)$, respectively. In addition, hepatic Tnf- $\alpha$ mRNA expression, induced by exposure to LPS or IRI+LPS, was normalised by macrophage depletion (Figure 4.2A). Mcp-1 expression, crucial for the recruitment of monocytes and macrophages to areas of inflammation and markedly induced following LPS administration, was comparable to controls when LPS groups were pretreated with clodronate liposomes (Figure 4.2B).
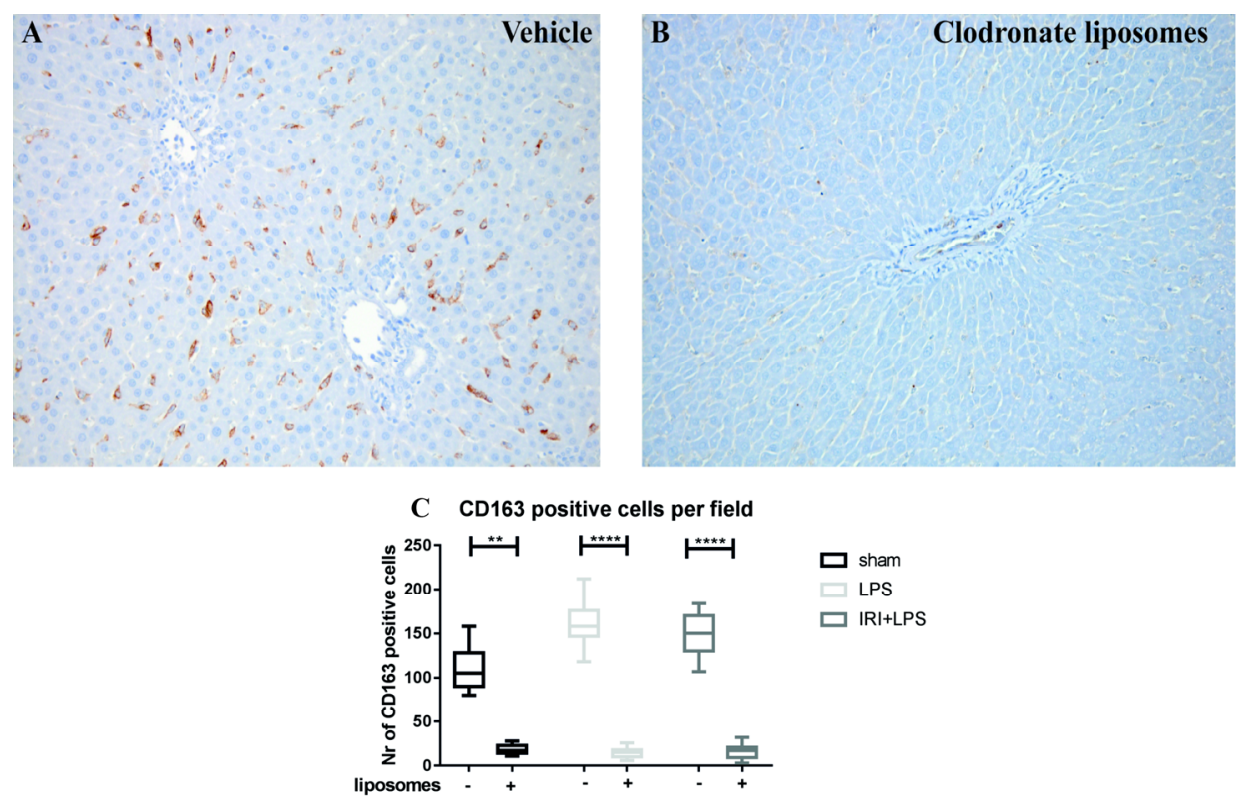

Figure 4.1 Successful depletion of mature macrophages following clodronate liposome treatment. Groups of rats were pre-treated during 48 hours with vehicle or clodronate liposomes, followed by sham operation, administration of lipopolysaccharides (LPS), or a combination of LPS and 30 minutes warm hepatic ischaemia (IRI+LPS). Animals were sacrificed after 6 hours of reperfusion. Representative light microscopy images of liver sections (200x magnification) stained for CD163 are depicted for an animal receiving vehicle (A) or clodronate liposomes (B). Quantification of $\mathrm{CD} 163^{+}$cells per high-powered field (C). Data is graphed using box plots presenting median, minimum and maximum values. Statistical significance was evaluated using a Kruskal-Wallis test, and -if appropriate- three predefined post-hoc comparisons (sham versus liposomes+ sham, LPS versus liposomes+LPS and IRI+LPS versus liposomes+IRI+LPS). $* * p<0.01, * * * * p<0.0001$. 

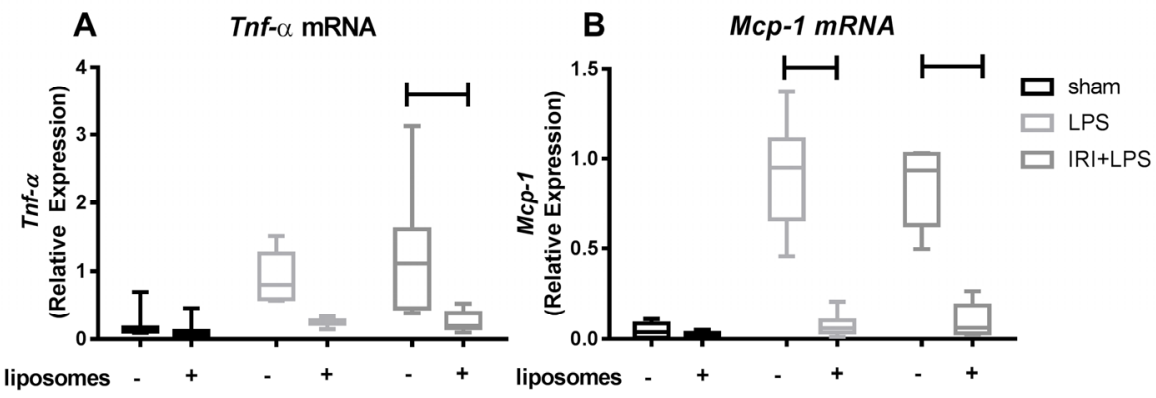

Figure 4.2 Decreased hepatic expression of genes related to inflammation following clodronate liposome treatment. Groups of rats were pre-treated during 48 hours with vehicle or clodronate liposomes, followed by sham operation, administration of lipopolysaccharides (LPS), or a combination of LPS and 30 minutes warm hepatic ischaemia (IRI+LPS). Animals were sacrificed after 6 hours of reperfusion, and hepatic mRNA expression was analysed (left lateral and median segments) using RTqPCR. Hepatic mRNA expression of Tnf- $\alpha(\mathrm{A})$ and Mcp-1 (B). Data is graphed using box plots presenting median, minimum and maximum values. Statistical significance was tested using Kruskal-Wallis multiple comparison test. Statistical significance was evaluated using a Kruskal-Wallis test, and -if appropriate- the three predefined post-hoc comparisons. ${ }^{* *} p<0.01$.

\section{Macrophage depletion causes elevated liver tests}

Serum ALT activity was not affected by macrophage depletion (Figure 4.3A). However, AST was significantly increased in the macrophage-depleted groups compared to their non-depleted controls (sham: $p=0.001$, LPS: $p=0.03$ and IRI+LPS $p=0.003$, Figure 4.3B). ALP and GGT were both significantly increased following macrophage depletion in the LPS-treated groups (Suppl. Data Figure S4.1). LPS-induced hyperbilirubinemia was ameliorated in the absence of macrophages (Figure 4.3C). On a transcriptional level, this was associated with a trend towards increased gene expression of ATP-binding cassette protein Mrp2, which facilitates the biliary secretion of glucuronidated bilirubin (Figure 4.3E). Following macrophage depletion, the serum elevation of bile salts tended to improve in the LPS group $(p=0.06)$ but remained elevated in the IRI+LPS group (Figure 4.3D). The latter occurred despite a significant increase in all groups ( $p<0.01$ for all comparisons, Figure 4.3F) of $\mathrm{Na}^{+}$-taurocholate co-transporting polypeptide (Ntcp), the transporter responsible for basolateral uptake of conjugated bile salts. 

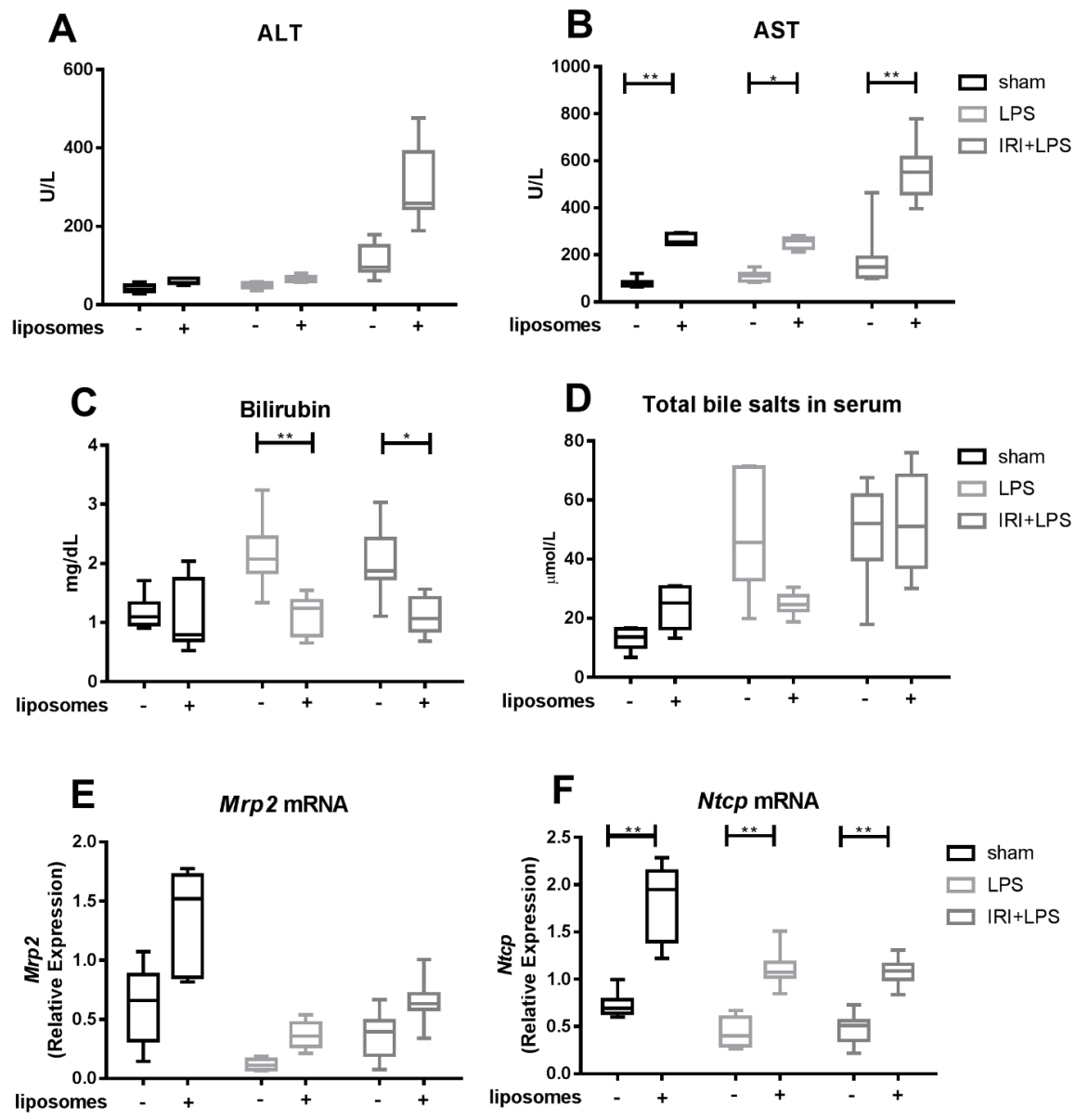

Figure 4.3 Serum liver tests. Groups of rats were pre-treated during 48 hours with vehicle or clodronate liposomes, followed by sham operation, administration of lipopolysaccharides (LPS), or a combination of LPS and 30 minutes warm hepatic ischaemia (IRI+LPS). Animals were sacrificed after 6 hours of reperfusion and serum activity or level of A) alanine transaminase (ALT), B) aspartate transaminase (AST), C) bilirubin and D) bile salts $(\mu \mathrm{mol} / \mathrm{L})$ were determined. Furthermore, hepatic mRNA expression of Mrp2 (B) and Ntcp (C) was measured using realtime quantitative polymerase chain reaction. ${ }^{*} p<0.05,{ }^{*} p<0.01$.

\section{Effective macrophage depletion did not result in reduced small bile duct injury}

A small bile duct injury severity score (SBDISS) was calculated, and representative portal tract images are depicted in Figure 4.4A. None of the animals developed severe small bile duct injury in the sham groups. LPS administration induced severe small bile 
duct injury (SBDISS $>4$ ) in 5 (62.5\%) animals compared to $6(75 \%)$ in the liposomes+LPS group. In the IRI+LPS group, severe small bile duct injury occurred in 5 (62.5\%) animals compared to $4(50 \%)$ in the liposomes+IRI+LPS group. Macrophage depletion did not alter LDH activity in bile, a biomarker for biliary injury, ${ }^{24}$ following LPS administration (Figure 4.4B). ${ }^{24}$ Although biliary LDH activity was slightly increased in the liposomes+sham group compared to sham controls, this did not reach significance $(p=0.09)$.
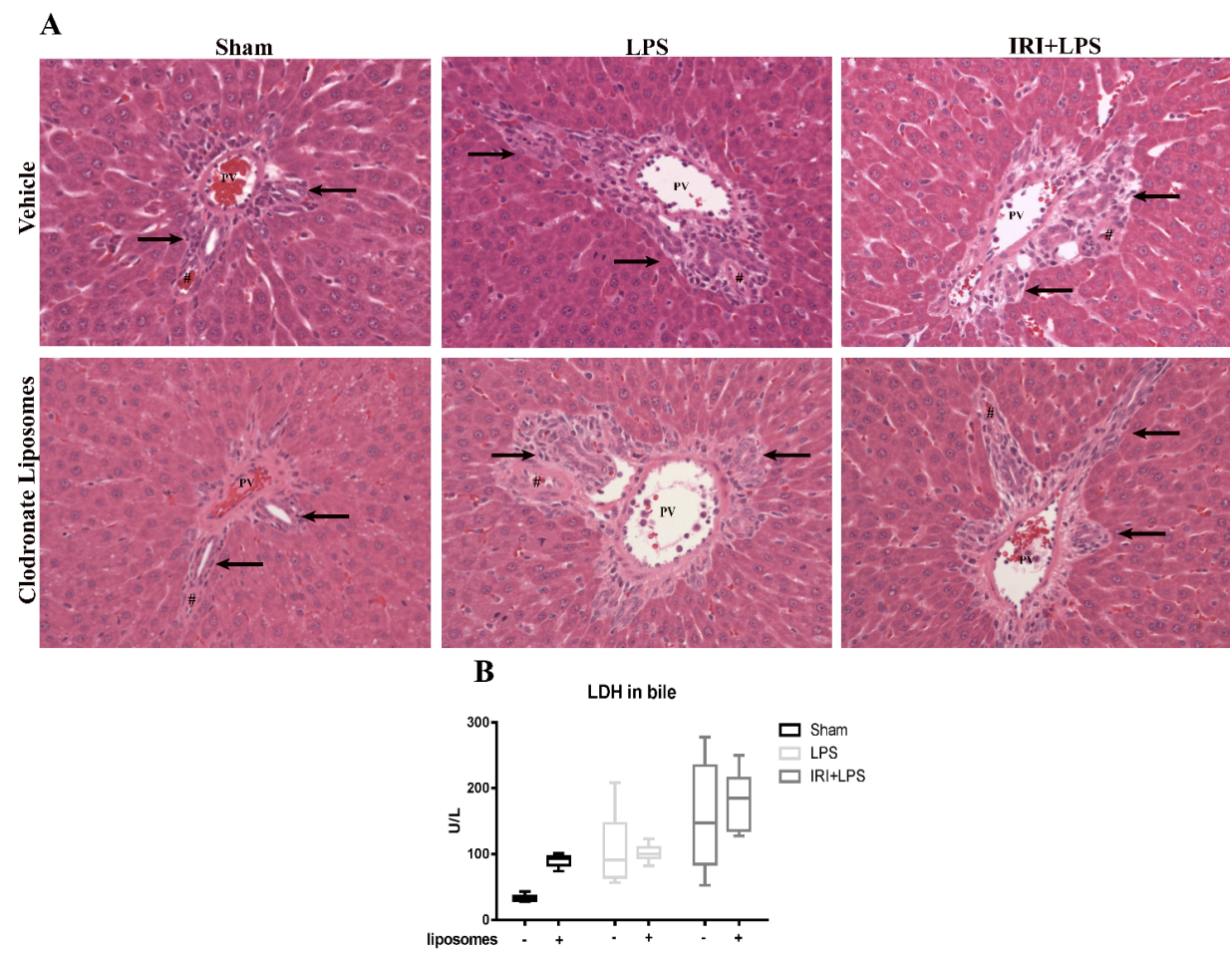

Figure 4.4 Macrophage depletion did not ameliorate LPS induced biliary injury. A) Representative light microscopy images of portal tracts (magnification 200x) with arrow indicating bile ducts, PV: portal vein, \$ hepatic artery. B) Concentration of lactate dehydrogenase (LDH) in bile as a biomarker of biliary injury.

\section{Persistent impairment of the BBB following macrophage depletion}

In the sham group, macrophage depletion led to a significant increase in the output of HRP in bile suggesting increased BBB permeability (Figure 4.5A). Elevated biliary HRP activity was not affected by macrophage depletion in the LPS and IR+LPS groups. We subsequently assessed gene expression levels of tight junction proteins Claudin-1 and 
zonula occludens-1 (Zo-1) (Figure 4.5B and 4.5C). Claudin-1 expression remained low in the liposomes+LPS group but was significantly increased in the liposomes +IR+LPS group. The expression did not exceed those of the untreated sham controls $(-1.4$ fold versus Sham). Zo-1 expression was not altered in the absence of macrophages in any of the groups.

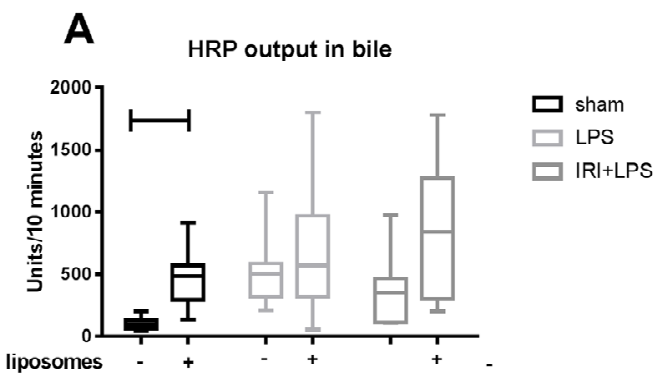

B

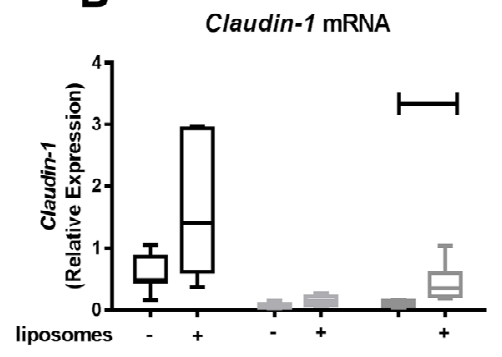

C

Zo-1 mRNA

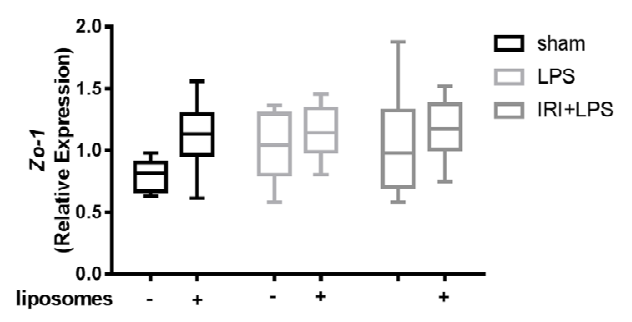

Figure 4.5 Persistent impairment of the blood biliary barrier following depletion of macrophages. A) Units of Horseradish peroxidase (HRP) output in bile during a 10-munte collection period following intravenous injection of 1000 IU HRP. Hepatic mRNA expression of B) Claudin-1 and C) Zo-1 measured using RT-qPCR. * $p<0.05$

\section{Macrophage depletion increased bile flow and total bile salt excretion}

Bile flow was increased following macrophage depletion, reaching significance in the LPS group, and total bile salt and phospholipid output were increased in the LPStreated groups (Figure 4.6A-C). This was accompanied by increased expression of the bile salt export pump (Bsep) in the LPS group (Figure 4.6D), but reduced levels of $M d r 2$, encoding the flippase that facilitates canalicular extraction of phospholipids, in both LPS-treated groups (Figure 4.6E). 

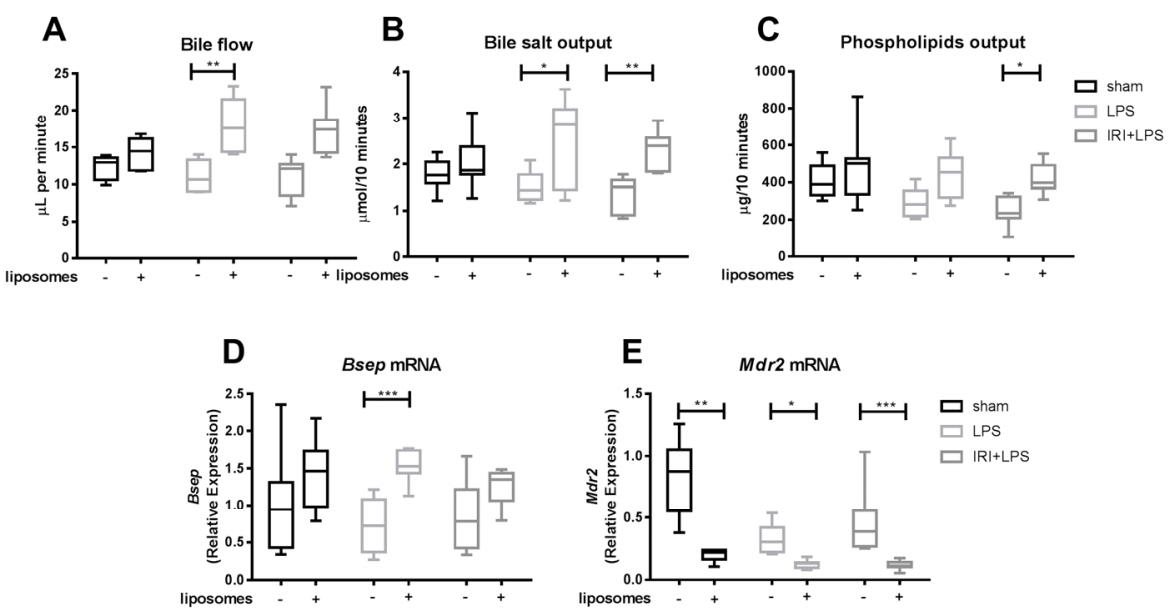

Figure 4.6 Bile flow, bile composition and gene expression of bile acid transporters. A) Bile flow $(\mu \mathrm{L} / \mathrm{min}), \mathrm{B})$ Total bile salt $(\mu \mathrm{mol})$ and $\mathrm{C})$ phospholipid $(\mu \mathrm{g})$ output in bile during a 10-minute collection period D) Hepatic mRNA expression of Bsep and E) Mdr2 determined by RT-qPCR. ${ }^{*} p<0.05,{ }^{* *} p<0.01,{ }^{* * *} p<0.001$.

\section{Cytokine production in bile remains largely unaltered}

The biliary concentration of several cytokines and chemokines were measured to determine the effect of macrophage depletion on the local inflammatory milieu surrounding cholangiocytes (Figure 4.7 and Supplementary Figure S4.2). Clodronate liposome treatment increased biliary Icam-1 protein in the LPS+IRI group to control levels (Figure 4.7A). Elevation of Mcp-1 in bile in the LPS groups, was not affected by macrophage depletion (Figure 4.7B). Timp-1 levels were elevated in the LPS-treated groups, and further increased upon macrophage depletion in the IRI+LPS group (Figure 4.7C). 

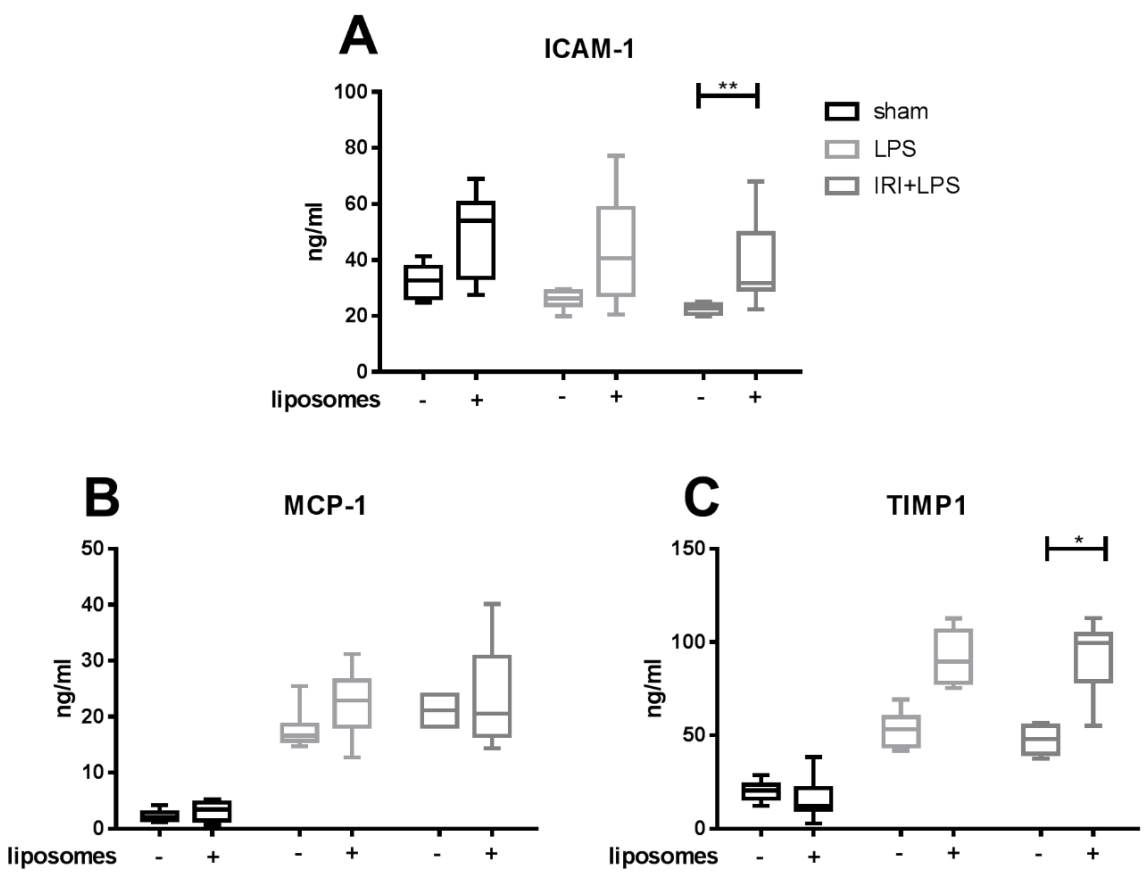

Figure 4.7 Pro-inflammatory cytokine and chemokine concentrations in bile. Biliary concentrations (ng/ml) of Icam-1 (A), Mcp-1 (B) and Timp-1 (C). * $p<0.05,{ }^{* *} p<0.01$.

\section{Discussion}

In the current study, clodronate liposomes were used to deplete macrophages from the liver. The development of biliary injury, resulting from exposure to LPS or LPSenhanced IRI, occurred independently of the presence of macrophages and impairment of the BBB persisted despite macrophage depletion. To our knowledge, this is the first study to explore the role of macrophages in the development of LPSinduced biliary injury.

In this study, $\mathrm{CD} 163^{+}$cells, a marker of mature macrophages, were largely depleted from the liver following treatment with liposomes. This observation was supported by a significant reduction in hepatic expression of pro-inflammatory cytokine Tnf- $\alpha$ and chemokine Mcp-1.

In the current study, serum elevations of AST, ALP and GGT were observed following clodronate liposome treatment. These findings differ from those previously reported. 
Macrophage depletion from donor livers using gadolinium chloride was found to be beneficial for early graft function and increased survival in animal studies. ${ }^{12}$ It furthermore reduced hepatic LPS toxicity and endotoxin-aggravated IRI. ${ }^{8,25}$ A possible explanation for the increased serum liver enzymes in this study, could be impaired clearance of the enzymes, a role recently attributed to Kupffer cells. ${ }^{26}$ This was further supported by absence of histological evidence of hepatocellular injury in the liposomes+sham group (data not shown).

Despite strong evidence that macrophages play an important role in the development of IRI and LPS-enhanced IRI, this study failed to show improvement in biliary injury following macrophage depletion. To determine the role of cholangiocytes in injury development we measured the biliary concentrations of several cytokines to assess the local inflammatory milieu surrounding cholangiocytes. Biliary Mcp-1 and Timp-1 protein levels were most strongly increased in the LPS and IRI+LPS groups. The presence of Mcp-1 in bile was not affected by macrophage depletion, whereas Timp-1 was more abundant in the clodronate liposome-treated groups. These findings suggest that macrophages only have a small effect on local biliary inflammatory milieu, which could explain the persistence of biliary injury in our model. Especially since TLR-4 signalling and subsequent production of cytokines by cholangiocytes, has been implicated in the pathophysiology of biliary diseases such as primary sclerosing cholangitis. $^{13}$

Another possible explanation for the development of biliary injury in the absence of macrophages could be a direct toxic effect of LPS on cholangiocytes. Cells from the reticulo-endothelial system together with hepatocytes are responsible for clearance of agents such as LPS from the portal circulation, and result in secretion of LPS in bile. $^{27-29}$ An accumulation of endotoxins has been observed in cholangiocytes of patients with primary biliary cholangitis and primary sclerosing cholangitis. ${ }^{30}$ Previous studies have shown that endotoxin clearance and secretion into bile can occur in the absence of macrophages, as hepatocytes are able to take up LPS. ${ }^{12,31}$ As cholangiocytes would remain exposed to LPS, this could explain why biliary injury developed in the macrophage-depleted groups.

The impairment of the BBB following exposure to LPS persisted in the absence of macrophages. Macrophages may play a role in maintaining the BBB as increased levels of HRP appeared in bile in the liposomestsham group. Tight junctions are the main structural components of the BBB, and its permeability can be tested using macromolecules (e.g. HRP) that cross the barrier from the blood stream via the paracellular route. ${ }^{21}$ Endotoxins as well as individual (pro-inflammatory) cytokines have previously been found to impair BBB function. ${ }^{32-34}$ Even though serum cytokine concentrations are likely reduced following macrophage depletion, the production of cytokines by cholangiocytes was maintained, which could have affected the BBB function. Alternatively, the increased concentration of HRP in bile observed in the 
liposomes+sham group could also be the result of reduced phagocytosis of HRP due to the absence of macrophages.

Endotoxaemia can induce impaired bile salt clearance via suppression of canalicular bile salt export (via Bsep) and basolateral bile salt uptake (via Ntcp). ${ }^{35,36}$ In the present study, we found that LPS-induced Ntcp suppression was reversed by macrophage depletion. This supports previous reports that macrophages play a key role in regulating hepatocytic Ntcp expression and portal venous bile salt clearance. ${ }^{36}$

A limitation of the study is the short duration of the injury, and the fact that cholangiocytes were not separated from other liver cell types for analyses. Cholangiocytes represent a small proportion of the total hepatic cell population, and total hepatic mRNA will in general provide little information on transcriptional changes in cholangiocytes. Furthermore, this study only assessed the short term effects of LPS administration or IRI, and further work is required to determine if biliary strictures would develop over time. The CD14/TLR4 signaling pathway has previously been linked to the development of ITBS following liver transplantation. ${ }^{37}$ In addition, a patient cohort study associated immunological factors with stricture formation in the periphery of the liver, affecting smaller bile ducts as was observed in this study. ${ }^{38,39}$ In conclusion, macrophage depletion did not prevent the development of biliary injury following LPS or LPS-enhanced IRI. Cholangiocytes can produce cytokines in a TLR4dependent fashion, and results from this study suggest that these cells themselves, rather than macrophages, may be key players in LPS-induced biliary injury. Studies investigating agents such as TLR4 or TNF-alpha signalling inhibitors in preventing LPSinduced biliary injury could provide additional information regarding the aetiology of LPS-induced biliary injury. 


\section{References}

1. Laing RW, Scalera I, Isaac J, Mergental H, Mirza DF, Hodson J, et al. Liver transplantation using grafts from donors after circulatory death: A propensity-matched study from a single centre. Am J Transplant. 2016.

2. Dubbeld J, Hoekstra H, Farid W, Ringers J, Porte RJ, Metselaar HJ, et al. Similar liver transplantation survival with selected cardiac death donors and brain death donors. Br J Surg. 2010;97(5):744-53.

3. Sun $\mathrm{Y}$, Hardaway RM, Williams $\mathrm{CH}$. Comparison of bacterial translocation during traumatic shock and hemorrhagic shock in rats. Int Surg. 1997;82(2):134-6.

4. Reiling J, Bridle, K., Santrampurwala, N., Britton, L., Campbell, C., Crawford, D., Dejong, C., Fawcett, J. Lipopolysaccharides increase bile toxicity and induce severe biliary injury in an LPS enhanced ischaemia-reperfusion model. HPB.18:e126-e7.

5. Nakamoto N, Kanai T. Role of toll-like receptors in immune activation and tolerance in the liver. Front Immunol. 2014;5:221.

6. Zhai Y, Petrowsky H, Hong JC, Busuttil RW, Kupiec-Weglinski JW. Ischaemia-reperfusion injury in liver transplantation--from bench to bedside. Nat Rev Gastroenterol Hepatol. 2013;10(2):79-89.

7. Caraceni P, Pertosa AM, Giannone F, Domenicali M, Grattagliano I, Principe A, et al. Antagonism of the cannabinoid CB-1 receptor protects rat liver against ischaemia-reperfusion injury complicated by endotoxaemia. Gut. 2009;58(8):1135-43.

8. Vajdova K, Smrekova R, Kukan M, Jakubovsky J, van Rooijen N, Horecky J, et al. Endotoxin-induced aggravation of preservation-reperfusion injury of rat liver and its modulation. J Hepatol. 2000; 32(1):112-20.

9. Pillay SP, Wynter C, Lynch S, Wall D, Balderson G, Strong R. Endotoxin levels in donors and recipients during orthotopic liver transplantation. Aust N Z J Surg. 1997;67(4):187-91.

10. Pillay SP, Moore TD, Lynch SV, Whiting RF, Balderson GA, Strong RW. Endotoxin levels in adult liver donors. Aust N Z J Surg. 1994;64(9):615-7.

11. Monbaliu D, van Pelt J, De Vos R, Greenwood J, Parkkinen J, Crabbe T, et al. Primary graft nonfunction and Kupffer cell activation after liver transplantation from non-heart-beating donors in pigs. Liver Transpl. 2007;13(2):239-47.

12. von Frankenberg M, Golling M, Mehrabi A, Nentwich H, Klar E, Kraus TW. Donor pretreatment with gadolinium chloride improves early graft function and survival after porcine liver transplantation. Transpl Int. 2003;16(11):806-13.

13. Syal G, Fausther M, Dranoff JA. Advances in cholangiocyte immunobiology. Am J Physiol Gastrointest Liver Physiol. 2012;303(10):G1077-86.

14. Harada K, Ohira S, Isse K, Ozaki S, Zen Y, Sato Y, et al. Lipopolysaccharide activates nuclear factorkappaB through toll-like receptors and related molecules in cultured biliary epithelial cells. Lab Invest. 2003;83(11):1657-67.

15. Harada K, Isse K, Nakanuma Y. Interferon gamma accelerates NF-kappaB activation of biliary epithelial cells induced by Toll-like receptor and ligand interaction. J Clin Pathol. 2006;59(2):184-90.

16. Ayres RC, Neuberger JM, Shaw J, Joplin R, Adams DH. Intercellular adhesion molecule-1 and MHC antigens on human intrahepatic bile duct cells: effect of pro-inflammatory cytokines. Gut. 1993; 34(9):1245-9.

17. Van Rooijen N, Kors N, vd Ende M, Dijkstra CD. Depletion and repopulation of macrophages in spleen and liver of rat after intravenous treatment with liposome-encapsulated dichloromethylene diphosphonate. Cell Tissue Res. 1990;260(2):215-22.

18. Van Rooijen N, Sanders A. Liposome mediated depletion of macrophages: mechanism of action, preparation of liposomes and applications. J Immunol Methods. 1994;174(1-2):83-93.

19. Schneider CA, Rasband WS, Eliceiri KW. NIH Image to ImageJ: 25 years of image analysis. Nat Methods. 2012;9(7):671-5.

20. Takakuwa Y, Kokai Y, Sasaki K, Chiba H, Tobioka H, Mori M, et al. Bile canalicular barrier function and expression of tight-junctional molecules in rat hepatocytes during common bile duct ligation. Cell Tissue Res. 2002;307(2):181-9. 
21. Lowe PJ, Kan KS, Barnwell SG, Sharma RK, Coleman R. Transcytosis and paracellular movements of horseradish peroxidase across liver parenchymal tissue from blood to bile. Effects of alphanaphthylisothiocyanate and colchicine. Biochem J. 1985;229(2):529-37.

22. Han Y, Glaser S, Meng F, Francis H, Marzioni M, McDaniel K, et al. Recent advances in the morphological and functional heterogeneity of the biliary epithelium. Exp Biol Med (Maywood). 2013;238(5):549-65.

23. Cheng L, Zhao L, Li D, Liu Z, Chen G, Tian F, et al. Role of cholangiocyte bile Acid transporters in large bile duct injury after rat liver transplantation. Transplantation. 2010;90(2):127-34.

24. Vajdova K, Smrekova R, Kukan M, Lutterova M, Wsolova L. Bile analysis as a tool for assessing integrity of biliary epithelial cells after cold ischemia--reperfusion of rat livers. Cryobiology. 2000;41(2):145-52.

25. limuro $\mathrm{Y}$, Yamamoto $\mathrm{M}$, Kohno H, Itakura J, Fujii H, Matsumoto Y. Blockade of liver macrophages by gadolinium chloride reduces lethality in endotoxemic rats--analysis of mechanisms of lethality in endotoxaemia. J Leukoc Biol. 1994;55(6):723-8.

26. Pervin M, Golbar HM, Bondoc A, Izawa T, Kuwamura M, Yamate J. Immunophenotypical characterization and influence on liver homeostasis of depleting and repopulating hepatic macrophages in rats injected with clodronate. Exp Toxicol Pathol. 2016;68(2-3):113-24.

27. Freudenberg MA, Galanos C. Bacterial lipopolysaccharides: structure, metabolism and mechanisms of action. Int Rev Immunol. 1990;6(4):207-21.

28. Hopf U, Ramadori G, Moller B, Galanos C. Hepatocellular clearance function of bacterial lipopolysaccharides and free lipid A in mice with endotoxic shock. Am J Emerg Med. 1984;2(1):13-9.

29. Mimura Y, Sakisaka S, Harada M, Sata M, Tanikawa K. Role of hepatocytes in direct clearance of lipopolysaccharide in rats. Gastroenterology. 1995;109(6):1969-76.

30. Sasatomi K, Noguchi K, Sakisaka S, Sata M, Tanikawa K. Abnormal accumulation of endotoxin in biliary epithelial cells in primary biliary cirrhosis and primary sclerosing cholangitis. J Hepatol. 1998;29(3):409-16.

31. Scott MJ, Billiar TR. Beta2-integrin-induced p38 MAPK activation is a key mediator in the CD14/TLR4/MD2-dependent uptake of lipopolysaccharide by hepatocytes. J Biol Chem. 2008;283(43):29433-46.

32. Sheth $P$, Delos Santos N, Seth A, LaRusso NF, Rao RK. Lipopolysaccharide disrupts tight junctions in cholangiocyte monolayers by a c-Src-, TLR4-, and LBP-dependent mechanism. Am J Physiol Gastrointest Liver Physiol. 2007;293(1):G308-18.

33. Hanada S, Harada M, Koga H, Kawaguchi T, Taniguchi E, Kumashiro R, et al. Tumor necrosis factoralpha and interferon-gamma directly impair epithelial barrier function in cultured mouse cholangiocytes. Liver Int. 2003;23(1):3-11.

34. Capaldo CT, Nusrat A. Cytokine regulation of tight junctions. Biochim Biophys Acta. 2009;1788(4):86471.

35. Hojo M, Sano N, Takikawa H. Effects of lipopolysaccharide on the biliary excretion of bile acids and organic anions in rats. J Gastroenterol Hepatol. 2003;18(7):815-21.

36. Sturm E, Havinga R, Baller JF, Wolters H, van Rooijen N, Kamps JA, et al. Kupffer cell depletion with liposomal clodronate prevents suppression of Ntcp expression in endotoxin-treated rats. J Hepatol. 2005;42(1):102-9.

37. Friedrich K, Smit M, Brune M, Giese T, Rupp C, Wannhoff A, et al. CD14 is associated with biliary stricture formation. Hepatology. 2016.

38. op den Dries S, Buis Cl, Adelmeijer J, Van der Jagt EJ, Haagsma EB, Lisman T, et al. The combination of primary sclerosing cholangitis and CCR5-Delta32 in recipients is strongly associated with the development of nonanastomotic biliary strictures after liver transplantation. Liver Int. 2011;31(8):1102-9.

39. Op den Dries S, Sutton ME, Lisman T, Porte RJ. Protection of bile ducts in liver transplantation: looking beyond ischemia. Transplantation. 2011;92(4):373-9. 


\section{Supplementary material}

Table S4.1 Primer sequences used for real time RT-PCR.

\begin{tabular}{llll}
\hline Gene & Forward & Reverse & Gene ID \\
\hline Gapdh & tcctgcaccaccaactgcttagc & gcctgcttcaccaccttcttgat & NM_017008.4 \\
Btf3 & ccaaacaatctgtggatgga & tctgccaagtgtcaccttctt & NM_001008309.1 \\
Beta 2 microglobulin & cctgtgtgcggtttcatc & ttctgccttggagtcctttc & NM_012512.2 \\
Cyp7a1 & caagacgcacctcgctattc & gaggctgctttcattgcttc & NM_012942.2 \\
Cyp7b1 & tcactacagagccgccaga & gcaaggtgaccgcaaaga & NM_0191381 \\
Cyp8b1 & gatccgccgtggagataag & gccatcagggttgaggaa & NM_31241.1 \\
Claudin-1 & aagcaccgggcagataca & ccagcaggatgccaattac & NM_178847.3 \\
Zo-1 & cctccaaaacaggccattac & ttggtagctgagggcagaac & NM_031699.2 \\
Tnf-alpha & agatgtggaactggcagagg & cacgagcgggaatgagaa & X66539.1 \\
Mcp-1 & gatctgtgctgaccccaataa & cattcaaaggtgctgaagtcc & NM_031530.1 \\
Ntcp & tcaagtccaaaaggccaca & tgcccacattgatgacaga & NM_017047.1 \\
Mdr2 & tcagcaaccagagcagagaa & tgcccaggagcataaacaa & NM_012690.2 \\
Mrp2 & caagcaggtgttcgtcgtgt & acaggaggaactgtggcttg & NM_012833.2 \\
Bsep & gggcagtcacacccatctac & agcccaggatgacaaagaac & NM_031760.1 \\
\hline
\end{tabular}
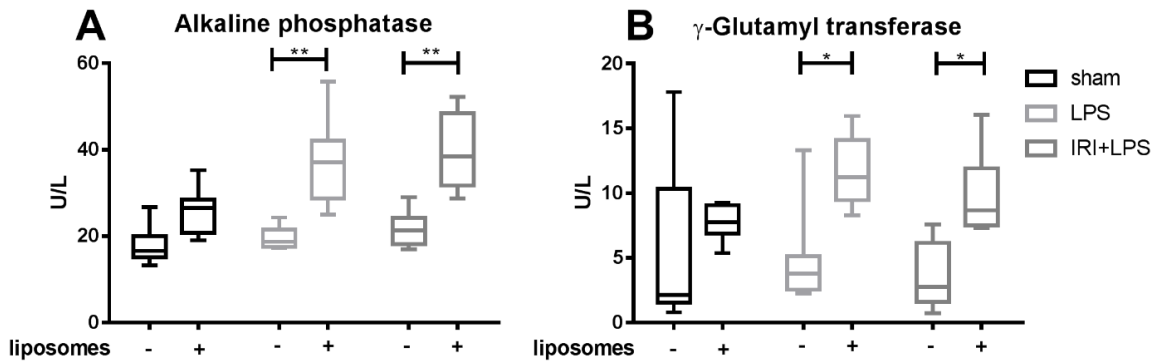

Figure S4.1 Serum liver tests. A) Serum activity of alkaline phosphatase (U/L) and B) $Y$-glutamyl transferase (U/L). Groups of rats were pre-treated during 48 hours with vehicle or clodronate liposomes, followed by sham operation, administration of lipopolysaccharides (LPS), or a combination of LPS and 30 minutes warm hepatic ischaemia (IRI+LPS). Animals were sacrificed after 6 hours of reperfusion. Data is graphed using box plots presenting median, minimum and maximum values. Statistical significance was evaluated using a Kruskal-Wallis test, and -if appropriate- three predefined post-hoc comparisons (sham versus liposomes+ sham, LPS versus liposomes+LPS and IRI+LPS versus liposomes+IRI+LPS).* $p<0.05,{ }^{* *} p<0.01$ 


\section{Chapter 5}

Comparable levels of inflammatory mediators in

portal venous blood collected from organ donors

donating after circulatory death and those donating

after brain death

J Reiling

DM Hohenhaus

MJ Sweet

AS Raj

CM Campbell

KR Bridle

N Santrampurwala

L Britton

DHG Crawford

CHC Dejong

J Fawcett

Submitted 


\section{Abstract}

\section{Background}

Extended criteria donors, such as those who donate after cardiac death (DCD) are increasingly considered for transplantation. Biliary stricture formation often complicates the use of these grafts and the aetiology remains largely unknown. Endotoxins, in the form of lipopolysaccharides (LPS) are potent inducers of biliary injury. However, it remains unclear if endotoxaemia occurs during DCD organ donation. The aim of this study was to determine the inflammatory propensity of portal blood collected from DCD donors compared to those donating after brain death (DBD).

\section{Method}

Serial portal venous, as well as hepatic venous, blood samples were collected from adult DBD and DCD organ donors. An NF-KB-dependent cell-based assay as well as a limulus amebocyte lysate quantification method was used to detect endotoxins and/or other inflammatory stimuli in the portal blood samples. $\beta$-galactosidase activity in hepatic venous samples was assessed as a marker for hepatic Kupffer cell activation. In addition, bile and bile duct tissue was collection for assessment of biliary injury.

\section{Key findings}

Thirty patients (nine DCD, 21 DBD) were included in this study. Compared to DBD donors, portal samples of DCD donors did not have an enhanced propensity for triggering inflammatory responses. However, Kupffer cell activation was enhanced and prolonged in DCD donors. Lactate dehydrogenase as a biomarker of biliary injury was increased in bile collected from DCD donors and histological scoring showed evidence of increased injury of peri-luminal peribiliary glands.

\section{Conclusion}

In this study no evidence was found of endotoxaemia or increased propensity to produce an inflammatory response in portal blood collected from DCD donors. Despite this, hepatic Kupffer cell activation was increased and there was evidence of biliary injury. 


\section{Introduction}

In the current era of organ shortage, an increasing number of extended criteria donors, such as those who donate after cardiac death (DCD) are considered for transplantation. Although equivalent short term outcomes have been reported by single centres, the use of DCD organs has been associated with inferior long term graft survival, increased need for biliary interventions and ultimately re-transplantation compared to livers donated after brain death (DBD). ${ }^{1,2}$ One of the main reasons for reduced graft survival after DCD liver transplantation is the development of ischaemic type biliary strictures (ITBS) in up to $30 \%$ of recipients. ${ }^{3,4}$ A better understanding of the pathophysiology of ITBS is urgently required in order to increase the use of these extended criteria donor organs.

In DCD organ donation, a period of warm ischaemia and hypo-perfusion occurs following withdrawal of life support prior to the commencement of cold perfusion. Bacterial translocation as well as endotoxins and danger- associated molecular patterns (DAMPs) are released into the portal circulation during gut ischaemia, and could potentially be flushed through the liver upon initiation of cold perfusion via the aorta during organ retrieval. ${ }^{5}$ Previous studies conducted in our laboratory showed that endotoxin, in the form of lipopolysaccharides (LPS). is a potent inducer of biliary injury. ${ }^{6}$ In the biliary injury groups, rats showed impairment of the blood biliary barrier as well as clinical signs of cholestasis. In addition, the Toll like receptor-4 (TLR-4) signalling pathway, via which LPS and some DAMPs signal, has recently been linked to the development of ITBS following transplantation. ${ }^{7}$

The deleterious consequences of endotoxaemia in the donor have previously been shown to be transferable to the recipient. Livers from rats pre-treated with LPS performed much more poorly during machine perfusion than non-pre-treated controls as evidenced by decreased portal flow, decreased bile production and increased lactate dehydrogenase levels in the perfusate. ${ }^{8}$ Furthermore, livers retrieved from organ donors with plasma LPS concentrations $>12 \mathrm{pg} / \mathrm{ml}$ failed more frequently compared to donors with LPS concentrations below that threshold. ${ }^{9}$ Endotoxaemia in portal and systemic circulation was shown to occur during organ retrieval of DBD donors ${ }^{10}$ as well as during transplantation ${ }^{11}$ but formal evidence of increased levels of inflammatory mediators in portal blood of DCD donors is currently lacking.

The aim of this study was to compare the levels of inflammatory mediators in portal venous blood collected from DCD and DBD donors during organ retrieval. We furthermore aimed to assess the effect of inflammatory mediators on the development of bile duct injury. We hypothesized that portal blood of DCD donors has an enhanced propensity for triggering inflammatory responses in the donor liver. 


\section{Methods}

\section{Organ donors}

All organ donors over the age of 18 who did not have hepatitis B, C or HIV, were eligible for inclusion in this study. Informed consent to collect samples for research purposes was obtained from donor families and the Human Research Ethics Committee of the Princess Alexandra Hospital as well as The University of Queensland, Brisbane, Australia, approved the study protocol.

For the purpose of this study, baseline donor characteristics such as age, sex, body mass index (BMI) and liver function tests prior to organ retrieval were collected. Additional parameters that could potentially induce or indicate an inflammatory state such as cause of death, signs of infection, use of antibiotics, period of downtime (absence of circulation prior to successful cardio-pulmonary resuscitation), and white cell count were also gathered.

\section{Organ retrieval and sample collection}

Organ retrieval was performed in a standardised fashion. Cannulas were inserted in the aorta as well as the portal vein and cold perfusion with University of Wisconsin (UW) solution was initiated. During DBD organ donation, 25,000 IU of heparin as well as $250 \mathrm{mg}$ of chlorpromazine, to induce vasodilatation, was administered prior to cross clamp. In DCD donation, local legislation does not permit the administration of heparin ante-mortem and therefore 50,000 IU of heparin was added to the first litre of cold perfusate.

To facilitate portal venous sample collection for the purpose of this study, an intravenous catheter was inserted in the portal venous system. In the majority of cases, the blood collection catheter was inserted in the superior mesenteric vein as a cannula was already inserted in the splenic vein or inferior mesenteric vein to facilitate cold perfusion. During organ retrieval, several blood samples from both the portal venous system as well as from the hepatic veins were collected using $10 \mathrm{ml}$ disposable syringes (pyrogen free) (Figure 5.1). The samples were then transferred to sterile blood collection tubes containing $17 \mathrm{IU} / \mathrm{ml}$ lithium heparin and stored on ice until further processing in the laboratory. In addition to the blood samples, bile and well as common bile duct tissue were collected. 
Start of cold

perfusion via aorta end of cold

perfusion via aorta

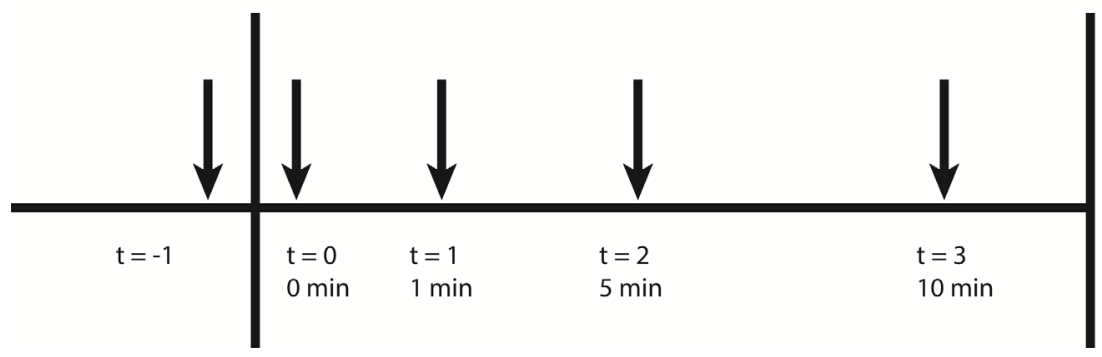

Figure 5.1 Blood sample collection during organ retrieval. Samples were collected at several time points during the organ retrieval process. The first sample was collected prior to the start of cold perfusion ( $t=-1)$. Subsequent samples were collected at the start of cold perfusion $(t=0)$ and 1 $(t=1), 5(t=2)$ and 10 minutes $(t=3)$ thereafter.

Kinetic quantitative chromogenic Limulus amebocyte lysate assay

Blood samples collected during organ retrieval were centrifuged at $3000 \mathrm{~g}$ for 5 minutes after which plasma was removed and aliquoted in sterilised endotoxin free $1.5 \mathrm{ml}$ micro-centrifuge tubes. This procedure was conducted in a class II biosafety cabinet using sterile endotoxin free tips (GoldenGate Bioscience, Claremont, CA, USA). The samples were then stored at $-80^{\circ} \mathrm{C}$ until further processing.

A limulus amebocyte lysate ( $L A L)$ kinetic chromogenic assay (Endochrome $\mathrm{K}$, Charles River, SC, USA) was performed in a subset of organ donors to determine the concentration of endotoxins in portal samples. In short, portal plasma samples were diluted in endotoxin-free water (LAL reagent water, Charles River, SC, USA) and heated to $75^{\circ} \mathrm{C}$ for 20 minutes. The samples were then further diluted in pyrogen free water (final dilution 1: 20). A standard curve ranging from $0.5 \mathrm{EU} / \mathrm{ml}$ to $0.001 \mathrm{EU} / \mathrm{ml}$ was prepared from serial dilutions of a stock $50 \mathrm{EU} / \mathrm{mL}$ standard (Control Standard Endotoxin, Charles River, SC, USA).

Each sample was tested in quadruplicate and $100 \mu \mathrm{l}$ of sample was administered to each well. Two wells per sample were spiked with $0.5 \mathrm{EU} / \mathrm{ml}$ of control standard endotoxin to determine the presence of inhibitory or enhancing factors. Finally, $100 \mu \mathrm{l}$ of LAL-reagent was added and the concentration of endotoxin in each sample was derived from optical density measurements with reference to the standard curve using a Tecan Sunrise ${ }^{\mathrm{TM}}$ plate reader and Endoscan ${ }^{\mathrm{TM}}$ Version $\mathrm{V}$ software (Charles River, SC, USA). Endotoxin-free water (LAL reagent water, Charles River, SC, USA) and University of Wisconsin solution were used as negative controls, and plasma from a subject with known gram-negative septicaemia was used as a positive control. 
Accounting for a 1:20 dilution of the samples, the lowest detectable level of endotoxin was $0.02 \mathrm{EU} / \mathrm{ml}$. The entire process was conducted in a class II biosafety cabined using endotoxin-free borosilicate glass tubes (Charles River, SC, USA) and endotoxin-free pipette tips (Eppendorf ${ }^{\circledR}$ BioPur Pipette Tips, Lonza, USA).

\section{Assessment of inflammatory capacity}

From DBD donors, samples collected at $\mathrm{t}=0$ were assayed to determine their propensity to induce an inflammatory response. From DCD donors, the earliest available sample was tested (range 1-14 minutes after the start of cold perfusion).

An NF-kB-dependent cell-based assay was used to determine the presence of proinflammatory mediators in portal blood samples, as previously described (12). In short, $2 \times 10^{5}$ ELAM9 cells (RAW264.7 murine macrophage-like cells stably transfected with human E-selectin promoter driving green fluorescent protein, hereafter ELAM cells) were plated in 24 well plates and allowed to adhere overnight in $1 \mathrm{ml}$ media (RPMI 1640 plus 10\% serum supreme, Invitrogen, San Diego, CA, USA), $20 \mathrm{U} / \mathrm{ml}$ penicillin and $20 \mu \mathrm{g} / \mathrm{ml}$ streptomycin. The following day, $460 \mu \mathrm{l}$ of fresh media was applied to the cells and $40 \mu \mathrm{l}$ of sample was added to each well. As a positive control, $10 \mathrm{ng} / \mathrm{mL}$ of LPS (Salmonella enterica serotype minnesota, Sigma-Aldrich, St Louis, MO, USA) was added. Unstimulated ELAM cells served as negative controls. In addition, a sterile sample of UW solution was tested as some portal venous samples likely contained some preservation solution. ELAM cells were stimulated for a total of six hours after which they were washed twice with ice-cold PBS and harvested in icecold LIFT buffer (1 mM EDTA, 0.1\% Sodium Azide in PBS). Flow cytometry was subsequently performed using a FACS Canto II to assess expression of green fluorescent protein.

\section{Assessment of Kupffer cell activation}

Glycohydrolases such as $\beta$-galactosidase are lysosomal enzymes that are released upon Kupffer cell activation. ${ }^{13} \beta$ - Galactosidase in hepatic vein samples was measured using a fluorimetric assay as previously described by McGuire et al.. ${ }^{14}$ In short, $20 \mu \mathrm{L}$ of sample or standard were tested in duplicate on a 96-well microtiter plate (\#CLS3603, Corning Incorporated, Corning NY, USA). Sequentially diluted 4-methylumbelliferone $(100 \mu \mathrm{mol} / \mathrm{L}$ in citrate phosphate buffer, \#M1381, Sigma-Aldrich) was used to constitute a standard curve. To each well, $80 \mu \mathrm{L}$ of 4-methylumbelliferyl-gluctoside (3.33 mmol/L in citrate-phosphate buffer, \#M1381, Sigma-Aldrich) was added and the plate was sealed with adhesive film. Following incubation for 30 minutes at $37^{\circ} \mathrm{C}$, $200 \mu \mathrm{L}$ of glycine-sodium hydroxide buffer was added to each well and the fluorescence signal was measured at $350 \mathrm{~nm} / 485 \mathrm{~nm}$ excitation/emission respectively. 


\section{Assessment of biliary injury}

Lactate dehydrogenase (LDH) levels in bile were assessed as a biomarker for biliary injury using a commercially available enzymatic colorimetric assay (\#431-15001, Wako Pure Chemical Industries Ltd., Osaka, Japan). ${ }^{15}$ In addition, paraffin embedded common bile duct sections were stained with haematoxylin and eosin and assessed by an expert histopathologist in a semi-quantitative fashion as previously described by Hansen et al. and op den Dries et al.. ${ }^{16,17}$ The biliary epithelium, peribiliary glands as well as the vascular plexus were assessed and damage to each component was graded as follows: 0: no damage, $1:<50 \%$ of the duct affected, $2>50 \%$ of the duct affected. When arteriolonecrosis was present, a score of 3 was awarded. In addition to the above mentioned components, the presence of mural stromal necrosis was assessed and the following scoring was performed: 0 , no damage, $1:<25 \%$ of the duct affected, $2 ;<50 \%$ of the duct affected, $3:<75 \%$ of the duct affected, $4: 100 \%$ of the duct affected.

\section{Statistical analysis}

Continuous variables are reported as median (inter quartile range) and categorical variables as frequencies (\%). A chi-square test, Fisher's exact test or Mann-Whitney U test were performed where appropriate to compare the two organ donor groups. A Kruskal-Wallis test was performed to compare the results of the NF-KB-dependent cell-based assay. SPSS statistical analysis software (IBM Corp. released 2013. IMB SPSS statistics for windows, version 22.0 Armonk, NY: IMB corp.) was used to perform the analysis and a $p$-value of $<0.05$ was considered significant.

\section{Results}

\section{Organ donor characteristics}

Samples were collected from 30 adult organ donors (Table 5.1). Nine DCD donors and 21 DBD donors were included in the study; 17 (57\%) of the patients were male. For the majority of DCD donors, the liver was declined for transplantation and subsequently perfused under normothermic conditions for research purposes. All but one liver in the DBD group were successfully transplanted. DCD donors had an average age of 60 (40-62) years and had a significantly lower body mass index compared to DBD donors $\left(21(21-24) \mathrm{kg} / \mathrm{m}^{2}\right.$ versus $\left.27(22-33) \mathrm{kg} / \mathrm{m}^{2}, p=0.03\right)$. The length of stay prior to organ donation was comparable between the two groups with organ retrieval taking place on average $3(2-5)$ days following admission. Serum liver function tests prior to retrieval were similar between the two donor groups as was the number of white blood cells. 
Table 5.1 Donor characteristics.

\begin{tabular}{|c|c|c|c|c|}
\hline & $\begin{array}{c}\text { DCD } \\
(\mathrm{N}=9)\end{array}$ & $\begin{array}{c}\text { DBD } \\
(\mathrm{N}=21)\end{array}$ & $\begin{array}{c}\text { Total } \\
(\mathrm{N}=30)\end{array}$ & $p$-value \\
\hline Age, years & $60(40-62)$ & $49(32-63)$ & $50(36-62)$ & 0.6 \\
\hline Sex, male & $4(44 \%)$ & $13(62 \%)$ & $17(57 \%)$ & 0.5 \\
\hline $\mathrm{BMI}, \mathrm{kg} / \mathrm{m}^{2}$ & $21(21-24)$ & $27(22-33)$ & $24(21-30)$ & 0.03 \\
\hline Cause of death & & & & 0.09 \\
\hline Stroke & $4(44 \%)$ & $10(48 \%)$ & $14(47 \%)$ & \\
\hline Hypoxic brain injury & $3(33 \%)$ & $6(29 \%)$ & 9 (30\%) & \\
\hline Trauma & $0(0 \%)$ & $5(24 \%)$ & $5(17 \%)$ & \\
\hline Other & $2(22 \%)$ & $0(0 \%)$ & $2(7 \%)$ & \\
\hline Length of stay, days * & $5(2-8)$ & $3(2-4)$ & $3(2-5)$ & 0.2 \\
\hline Use of inotropes in ICU & $5(56 \%)$ & $14(67 \%)$ & $19(63 \%)$ & 0.7 \\
\hline Antibiotics & $5(56 \%)$ & $8(38 \%)$ & $13(43 \%)$ & 0.4 \\
\hline Period of downtime, min & $0(0-8)$ & $0(0-32)$ & $0(0-21)$ & 0.2 \\
\hline White blood cells, count & $14(10-21)$ & $11(7-16)$ & $12(8-16)$ & 0.3 \\
\hline ALT, U/L & $42(13-155)$ & $42(23-166)$ & $42(17-157)$ & 0.7 \\
\hline AST, U/L & $42(24-71)$ & $81(36-99)$ & $66(29-90)$ & 0.1 \\
\hline GGT, U/L & $24(15-80)$ & $28(15-94)$ & $28(15-87)$ & 0.7 \\
\hline$A L P, U / L$ & $71(40-93)$ & $56(40-71)$ & $56(41-73)$ & 0.5 \\
\hline WIT, $\min$ & $17(14-21)$ & - & $17(14-21)$ & n.d. \\
\hline
\end{tabular}

WIT: warm ischaemic time, defined as the period between the onset of significant ischaemia and the start of organ perfusion.

Portal venous blood collected from DBD and DCD donors had a similar propensity for triggering an inflammatory response

ELAM cells were stimulated with portal blood collected from both DBD and DCD donors, and green fluorescent protein (GFP) levels as a proxy for NF-KB-dependent ELAM promoter activity were measured using flow cytometry (Figure 5.2). Following six hours incubation with LPS, the promoter activity increased 28-fold compared to untreated control cells $(p<0.001)$. Sterile UW solution did not induce an inflammatory response, as compared to non-treated controls. Although ELAM cells treated with portal blood from DCD donors had a slightly increased promoter activity compared to cells treated with blood from DBD donors (mean fluorescence intensity 85 versus 79, $p=0.003$ ), the inflammatory response in both groups was not significantly different from the promoter activity levels of untreated controls (average fluorescence intensity 85). Likewise, no significant differences were found in the concentration of endotoxins measured using the LAL assay, with the majority of samples having levels of endotoxins that were below the limits of detection. 


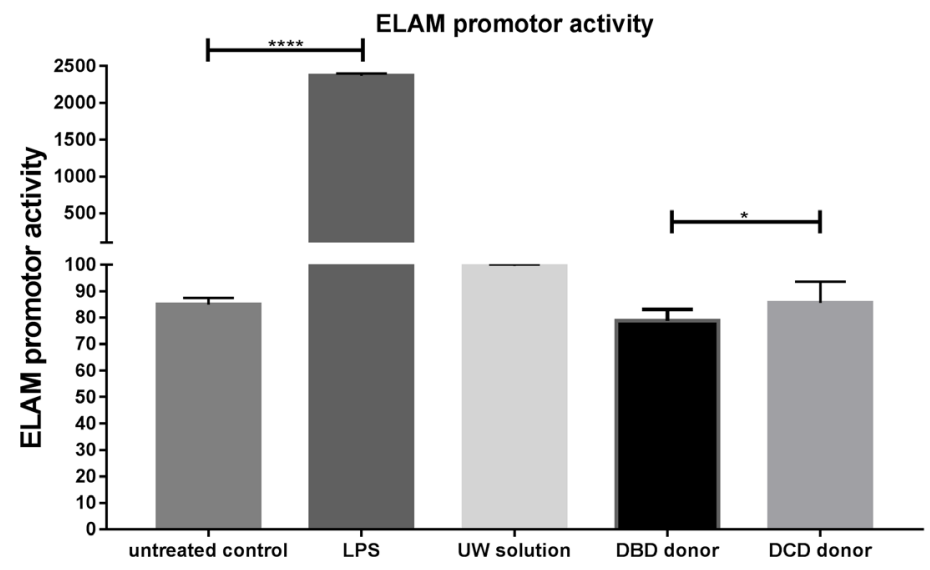

Figure 5.2 Unaltered ELAM promoter activity following incubation with portal blood from both DBD and DCD donors. ELAM cells were incubated for six hours with portal blood collected from brain death donors (DBD) as well as those donating after circulatory death (DCD). Flow cytometry was subsequently performed to assess expression of green fluorescent protein. Each sample was assayed in duplicate and lipopolysaccharides served as a positive control. Data are depicted as median with interquartile range. A Kruskal Wallis test was performed to compare the treatment groups and a $p$-value $<0.05$ was considered significant. ${ }^{*} p<0.05$, $* * * * p<0.0001$ Abbreviations: UW: university of Wisconsin solution, DBD: donation after brain death, DCD: donation after circulatory death, LPS: lipopolysaccharide, GFP: green fluorescent protein.

\section{Kupffer cell activation in DCD donors}

Serial hepatic vein samples were collected from DBD and DCD donors and $\beta$-galactosidase activity was assessed to determine the degree of Kupffer cell activation in the liver (Figure 5.3). From DBD donors, the following samples were available: prior to start of cold perfusion $(n=18)$, at the start of cold perfusion $(n=21)$, 1 minute $(n=15), 5$ minutes $(n=15)$ and 10 minutes of cold perfusion $(n=16)$. During DBD organ retrieval, the activity level of $\beta$-galactosidase in samples collected prior to the start of cold perfusion were comparable to those collected at the start of cold perfusion (94 (81-166) $\mathrm{U} / \mathrm{ml}$ versus 79 (67-139) $\mathrm{U} / \mathrm{ml}, p>0.99)$ (Figure 5.3A). During organ retrieval, the levels rapidly decreased to $19(12-26) \mathrm{U} / \mathrm{ml}$ after 5 minutes and $14(1-31) \mathrm{U} / \mathrm{ml}$ after 10 minutes of cold perfusion $(p<0.001)$. One DBD organ donor became haemodynamically unstable just prior to the start of cold perfusion, which resulted in high levels of Kupffer cell activation ( 3 -fold compared to $t=-1$ ). Furthermore, a single DBD donor who was admitted for 12 days prior to organ retrieval had increased levels of $\beta$-galactosidase compared to other DBD donors ( $t=-1$ : $457 \mathrm{U} / \mathrm{ml}, \mathrm{T}=0: 663 \mathrm{U} / \mathrm{ml}, \mathrm{T}=3: 97 \mathrm{U} / \mathrm{ml})$. 
In DCD donors, hepatic vein samples were collected at the start of cold perfusion $(n=3), 1$ minute $(n=3), 5$ minutes $(n=5), 10$ minutes $(n=3)$ and 15 minutes after the start of cold perfusion $(n=1)$. Compared to samples collected from DBD donors at the start of cold perfusion, peak activity levels of $\beta$-galactosidase were significantly higher in the DCD group $(p=0.02)$ (Figure 5.3B). Furthermore, following 5 minutes of reperfusion, Kupffer cell activation was increased in DCD donors, as compared to DBD donors $(p<0.001)$ (Figure 5.3C).

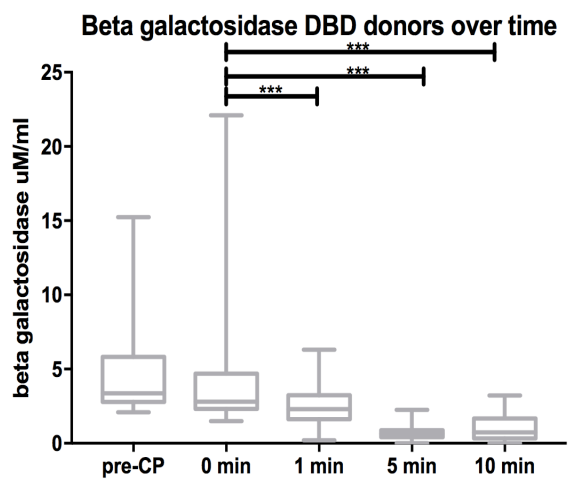

\section{Beta galactosidase DBD versus DCD donors}

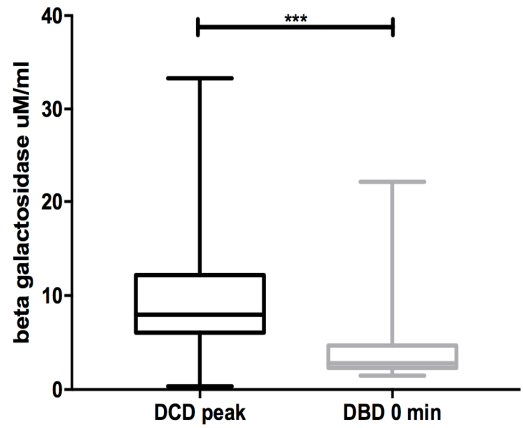

Beta galactosidase $D B D$ versus $D C D$ donors

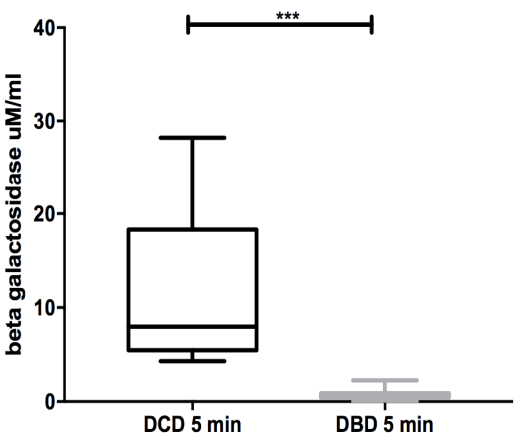

Figure 5.3 Kupffer cell during DCD organ retrieval. The activation of $\beta$-galactosidase was measured in hepatic vein samples as a surrogate marker of Kupffer cell activation. A) Levels of $\beta$-galactosidase $(\mathrm{U} / \mathrm{ml})$ during organ retrieval of livers donated after brain death (DBD). B) Comparison of $\beta$-galactosidase $(U / \mathrm{ml})$ levels between DBD donors and those donating after cardiac death $(D C D) C$ ) comparison of $\beta$-galactosidase $(U / \mathrm{ml})$ levels between $D B D$ and $D C D$ donors after 5 minutes of cold perfusion. Data are depicted as median, minimum and maximum using box and whisker plots. A Kruskal Wallis test was used to compare the concentrations at different time point amongst DBD donors whereas a Mann-Whitney $U$ test was used to compare activity levels between DBD and DCD donors. Abbreviations: pre-CP: before cross clamp, . ${ }^{* * *} p<0.001$. 


\section{Evidence of biliary injury in DCD donors}

LDH in bile, collected from the common bile duct of organ donors during organ retrieval, was measured as a biomarker of biliary injury (Figure 5.4). Compared to DBD donors, DCD donors had significantly higher levels of LDH in bile (3027 (782-5665) U/L versus 863 (471-1407) U/L, $p=0.04$ ). In addition, common bile duct histology sections were scored (Table 5.2). Overall, the biliary epithelium of the common bile duct was damaged in the majority of organs donors (25 (89\%)). The degree of damage was not significantly different between the two organ donor groups. The majority of common bile duct sections from DBD donors did not display any injury to the mural stroma. Although slightly more common bile duct (CBD) sections from DCD livers displayed mural necrosis, this did not reach significance. The grade of intramural bleeding, vascular lesions and inflammation were comparable between the two groups with the majority of common bile duct sections not showing any evidence of injury. However, the peri-luminal peribiliary glands were more severally affected in CBD sections of DCD donors compared to DBD donors. The grade of deep peribiliary gland damage was not different between DBD and DCD donors.

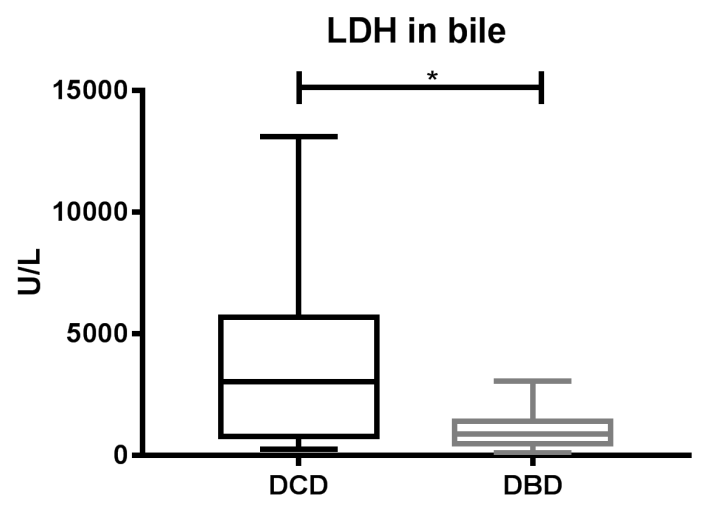

Figure 5.4 Lactate dehydrogenase in bile. The activity of lactate dehydrogenase (LDH, U/L) in bile was measured as a biomarker of biliary injury. A Mann-Whitney $U$ test was use to compare LDH levels in bile collected from brain death donors (DBD) as well as those donating after circulatory death (DCD). Data are depicted using a box and whisker plot. ${ }^{*} p 0.05$ 
Table 5.2 Bile duct histology scoring.

\begin{tabular}{|c|c|c|c|}
\hline & $\begin{array}{c}\text { DBD donor } \\
(\mathrm{N}=19)\end{array}$ & $\begin{array}{c}\text { DCD donor } \\
(\mathrm{N}=9)\end{array}$ & $p$-value \\
\hline Biliary epithelium & & & 0.2 \\
\hline Grade 0 & $2(11 \%)$ & $1(11 \%)$ & \\
\hline Grade 1 & $8(42 \%)$ & $1(11 \%)$ & \\
\hline Grade 2 & $9(47 \%)$ & $7(78 \%)$ & \\
\hline Necrosis of mural stroma & & & 0.3 \\
\hline Grade 0 & $14(74 \%)$ & $4(44 \%)$ & \\
\hline Grade 1 & $3(16 \%)$ & $3(33 \%)$ & \\
\hline Grade 2 & $2(11 \%)$ & $1(11 \%)$ & \\
\hline Grade 3 & $0(0 \%)$ & $1(11 \%)$ & \\
\hline Grade 4 & $0(0 \%)$ & $0(0 \%)$ & \\
\hline Intramural bleeding & & & 0.3 \\
\hline Grade 0 & $13(68 \%)$ & $9(100 \%)$ & \\
\hline Grade 1 & $4(21 \%)$ & $0(0 \%)$ & \\
\hline Grade 2 & $2(11 \%)$ & $0(0 \%)$ & \\
\hline Vascular lesions & & & 0.7 \\
\hline Grade 0 & $15(79 \%)$ & $9(100 \%)$ & \\
\hline Grade 1 & $3(16 \%)$ & $0(0 \%)$ & \\
\hline Grade 2 & $0(0 \%)$ & $0(0 \%)$ & \\
\hline Grade 3 & $1(5 \%)$ & $0(0 \%)$ & \\
\hline Inflammation & $\mathrm{N}=18$ & & 0.2 \\
\hline Grade 0 & $9(50 \%)$ & $8(89 \%)$ & \\
\hline Grade 1 & 7 (39\%) & $1(11 \%)$ & \\
\hline Grade 2 & $2(11 \%)$ & $0(0 \%)$ & \\
\hline Peri-luminal peribiliary glands & $N=18$ & & 0.01 \\
\hline Grade 0 & $10(56 \%)$ & $2(22 \%)$ & \\
\hline Grade 1 & $5(38 \%)$ & $0(0 \%)$ & \\
\hline Grade 2 & $3(17 \%)$ & $7(78 \%)$ & \\
\hline Deep peribiliary glands & $N=17$ & & 0.4 \\
\hline Grade 0 & $9(53 \%)$ & $3(33 \%)$ & \\
\hline Grade 1 & $8(47 \%)$ & $5(56 \%)$ & \\
\hline Grade 2 & $0(0 \%)$ & $1(11 \%)$ & 0.2 \\
\hline
\end{tabular}

\section{Discussion}

In this prospective patient cohort study, we showed no differences in portal venous levels of endotoxin between DBD and DCD organ donors. Despite apparently comparable levels of inflammatory mediators entering the liver, hepatic Kupffer cell activation was increased throughout the DCD organ donation process. Furthermore, more extensive biliary injury was present at the time of organ retrieval in DCD donors compared to their DBD counterparts.

In contrast to previous studies reporting translocation of endotoxins during shock ${ }^{18}$ and the interruption of portal and arterial blood flow, ${ }^{19}$ this study did not detect the presence of inflammatory mediators in portal blood collected from DCD donors - both 
by the direct measurement of endotoxin and also by the use of a bioassay for the presence of inflammatory stimuli. That trigger NF- $\kappa B$ activation. DCD organ donation is characterised by a period of hypo-perfusion and hypoxia and the results of this study were therefore unexpected and not consistent with previous reports in the literature.

In most previous reports, the concentration of endotoxins has been measured directly using a LAL assay or indirectly by determining the IgG endotoxin core antibodies. ${ }^{10,19}$ The LAL assay utilised was sensitive, to a lower limit of detection of $0.001 \mathrm{EU} / \mathrm{ml}$, however it is well recognised that assessment of endotoxin in plasma is affected by inhibitory factors such as serine proteases. ${ }^{20,21}$ Therefore an additional cell-based assay was performed in the current study. Although the NF-KB dependent reporter assay does not have the capacity to discriminate between pro-inflammatory mediators such as endotoxins and other stimuli that activate this key inflammatory signalling cascade, the results of this assay in the current study ruled out the involvement of any such mediator in sufficient concentrations to induce ELAM cell activation. As the assay is different from those ones in previous studies assessing the presence of endotoxins during organ donation, ${ }^{10}$ it is difficult to directly compare the results from that study with those from the present study.

The timing of sample collection in this study could be a possible factor limiting our ability to detect inflammatory mediators. In DCD donors, it was not always possible to collect portal venous samples at the start of aortic cold perfusion. As a result, the flush of cold perfusate through the aorta might potentially already have cleared the inflammatory mediators from the portal venous system. However, samples collected from DCD donors within 5 minutes of establishment of cold perfusion $(n=5)$ did not show higher levels of pro-inflammatory activity compared to samples collected at a later stage.

In the present study, Kupffer cell activation, as determined by the release of $\beta$-galactosidase, was increased during the DCD organ donation process. Unfortunately we were unable to obtain liver tissue for further assessment of Kupffer cells, but $\beta$ galactosidase has previously been used successfully in animal machine perfusion experiments as a surrogate marker for activation. ${ }^{22}$ Kupffer cells play a pivotal role in hepatic ischaemia-reperfusion injury (IRI). ${ }^{23}$ They release reactive oxygen species and pro-inflammatory cytokines upon activation resulting in the induction of hepatocellular injury. Increased levels of Kupffer cell activation have been associated with primary non-function following transplantation of porcine livers donated after circulatory death. ${ }^{22}$ Furthermore, Kupffer cells are also responsible for clearance of endotoxins from the portal circulation and endotoxaemia can significantly enhance IRI. $^{24}$

It was previously unclear to what extent endotoxaemia contributed to the development of IRI in DCD transplantation. Animal studies indicated that exposure of donors to endotoxins was associated with inferior graft outcome however evidence of 
endotoxaemia in DCD donation was lacking. ${ }^{8,25}$ Based on results of this study, Kupffer cell activation seems to be the result of hepatic ischaemia and local release of PAMPs since endotoxaemia was not observed during DCD organ donation.

In the current study, the concentration of LDH in bile was increased in DCD donors indicating more extensive biliary injury. Histological scoring revealed only mild evidence of bile duct injury in DCD donors with more severe injury of the luminal peribiliary glands. Although the bile duct injury found in our study might seem mild, a previous study conducted by Brunner et al. showed that the degree of injury progressed between organ retrieval and transplantation (26). Furthermore, as biliary LDH levels were increased in DCD donors, this might indicate more widespread injury to the biliary that was not captured in the CBD histology sections. Despite the early time point in this study, biliary injury could be detected in DCD donors at the time of organ retrieval. As most livers retrieved from DCD donors were not transplanted in this study, we were unable to assess whether these livers would have progressed to develop ITBS. Op den Dries et al. have previously found a strong correlation between the degree of mural stromal necrosis, injury to the peribiliary gland and the peribiliary plexus injury at the end of the cold storage period, and the development of ischaemic type biliary strictures following transplantation. ${ }^{16}$ It remains however, as to what extent the damage observed at the time of organ donation predicts ITBS formation.

Another factor that potentially plays a role in the progression of biliary injury is the exposure of injured cholangiocytes to endotoxins during transplantation. ${ }^{11}$ The cotoxicity of these two factors has been implicated in the development of secondary sclerosing cholangitis in critically ill patients (SSC-CIP). This rare disease is characterized by cholestasis, biliary cast and stricture formation and has a high mortality rate. ${ }^{27}$ Although ITBS does not develop or progress as rapidly as SSC-CIP, there are similarities between the two diseases with regards to their histological and radiological appearance. The exact role of endotoxaemia in the development of biliary injury following transplantation needs to be further assessed in future studies.

A limitation of the current study is the small sample size, which might have negatively impacted on our ability to show small differences between the study groups. Although smaller differences might have been found with a bigger sample size, these might not have had any clinical relevance. Furthermore, most of the livers obtained from DCD donors were not transplanted and therefore insufficient data are available on the development of biliary complications such as ITBS following transplantation.

In conclusion, this study showed that portal blood from DCD donors does not have the capacity to stimulate an enhanced NF- $\mathrm{KB}$-dependent pro-inflammatory response, as compared to DBD donors. Nevertheless, increased levels of Kupffer cell activation and biliary injury were found at the time of organ retrieval. Future studies focused on early intervention at retrieval are warranted to prevent the progression of biliary injury that has already developed at the time of organ retrieval. 


\section{References}

1. Jay CL, Lyuksemburg V, Ladner DP, Wang E, Caicedo JC, Holl JL, et al. Ischemic cholangiopathy after controlled donation after cardiac death liver transplantation: a meta-analysis. Ann Surg. 2011;253(2):259-64.

2. Blok JJ, Detry O, Putter H, Rogiers X, Porte RJ, van Hoek B, et al. Longterm results of liver transplantation from donation after circulatory death. Liver Transpl. 2016;22(8):1107-14.

3. Laing RW, Scalera I, Isaac J, Mergental H, Mirza DF, Hodson J, et al. Liver transplantation using grafts from donors after circulatory death: A propensity-matched study from a single centre. Am J Transplant. 2016.

4. Dubbeld J, Hoekstra H, Farid W, Ringers J, Porte RJ, Metselaar HJ, et al. Similar liver transplantation survival with selected cardiac death donors and brain death donors. Br J Surg. 2010;97(5):744-53.

5. Grootjans J, Lenaerts K, Derikx JP, Matthijsen RA, de BAP, van BAA, et al. Human intestinal ischemiareperfusion-induced inflammation characterized: experiences from a new translational model. Am J Pathol. 2010;176(5):2283-91.

6. Reiling J, Bridle, K., Santrampurwala, N., Britton, L., Campbell, C., Crawford, D., Dejong, C., Fawcett, J. Lipopolysaccharides increase bile toxicity and induce severe biliary injury in an LPS enhanced ischaemia-reperfusion model. HPB.18:e126-e7.

7. Friedrich K, Smit M, Brune M, Giese T, Rupp C, Wannhoff A, et al. CD14 is associated with biliary stricture formation. Hepatology. 2016.

8. Vajdova K, Smrekova R, Kukan M, Jakubovsky J, van Rooijen N, Horecky J, et al. Endotoxin-induced aggravation of preservation-reperfusion injury of rat liver and its modulation. J Hepatol. 2000;32(1): 112-20.

9. Zipfel A, Schenk M, You MS, Lauchart W, Bode C, Viebahn R. Endotoxemia in organ donors: graft function following liver transplantation. Transpl Int. 2000;13 Suppl 1:S286-7.

10. Pillay SP, Moore TD, Lynch SV, Whiting RF, Balderson GA, Strong RW. Endotoxin levels in adult liver donors. Aust N Z J Surg. 1994;64(9):615-7.

11. Abdala E, Baia CE, Mies S, Massarollo PC, de Paula Cavalheiro N, Baia VR, et al. Bacterial translocation during liver transplantation: a randomized trial comparing conventional with venovenous bypass vs. piggyback methods. Liver Transplant. 2007;13(4):488-96.

12. Stacey KJ, Young GR, Clark F, Sester DP, Roberts TL, Naik S, et al. The molecular basis for the lack of immunostimulatory activity of vertebrate DNA. J Immunol. 2003;170(7):3614-20.

13. Caldwell-Kenkel JC, Currin RT, Tanaka Y, Thurman RG, Lemasters JJ. Kupffer cell activation and endothelial cell damage after storage of rat livers: effects of reperfusion. Hepatology. 1991;13(1): 83-95.

14. McGuire JB, James TJ, Imber CJ, St Peter SD, Friend PJ, Taylor RP. Optimisation of an enzymatic method for beta-galactosidase. Clin Chim Acta. 2002;326(1-2):123-9.

15. Vajdova K, Smrekova R, Kukan M, Lutterova M, Wsolova L. Bile analysis as a tool for assessing integrity of biliary epithelial cells after cold ischemia--reperfusion of rat livers. Cryobiology. 2000;41(2):145-52.

16. op den Dries S, Westerkamp AC, Karimian N, Gouw AS, Bruinsma BG, Markmann JF, et al. Injury to peribiliary glands and vascular plexus before liver transplantation predicts formation of nonanastomotic biliary strictures. J Hepatol. 2014;60(6):1172-9.

17. Hansen $T$, Hollemann D, Pitton MB, Heise M, Hoppe-Lotichius $M$, Schuchmann $M$, et al. Histological examination and evaluation of donor bile ducts received during orthotopic liver transplantation--a morphological clue to ischemic-type biliary lesion? Virchows Archiv. 2012;461(1):41-8.

18. Chang JX, Chen S, Ma LP, Jiang LY, Chen JW, Chang RM, et al. Functional and morphological changes of the gut barrier during the restitution process after hemorrhagic shock. World J Gastroenterol. 2005;11(35):5485-91.

19. Dello SA, Reisinger KW, van Dam RM, Bemelmans MH, van Kuppevelt TH, van den Broek MA, et al. Total intermittent Pringle maneuver during liver resection can induce intestinal epithelial cell damage and endotoxemia. PLoS One. 2012;7(1):e30539. 
20. Dawson M. Interference with the LAL test and how to address it. LAL update, associates of cape cod, Inc. $2005 ; 22(3)$.

21. Ketchum PA, Novitsky TJ. Assay of endotoxin by limulus amebocyte lysate. Methods Mol Med. 2000;36:3-12.

22. Monbaliu D, van Pelt J, De Vos R, Greenwood J, Parkkinen J, Crabbe T, et al. Primary graft nonfunction and Kupffer cell activation after liver transplantation from non-heart-beating donors in pigs. Liver Transplant. 2007;13(2):239-47.

23. Kapoor S. Hepatic ischaemia-reperfusion injury from bench to bedside (Br J Surg 2010; 97: 14611475). Br J Surg. 2011;98(3):459; author reply -60.

24. Caraceni P, Pertosa AM, Giannone F, Domenicali M, Grattagliano I, Principe A, et al. Antagonism of the cannabinoid CB-1 receptor protects rat liver against ischaemia-reperfusion injury complicated by endotoxaemia. Gut. 2009;58(8):1135-43.

25. Azoulay D, Astarcioglu I, Lemoine A, Dennison A, Mathieu D, Saulnier C, et al. The effects of donor and recipient endotoxemia on TNF alpha production and mortality in the rat model of syngenic orthotopic liver transplantation. Transplantation. 1995;59(6):825-9.

26. Brunner SM, Junger $H$, Ruemmele $P$, Schnitzbauer AA, Doenecke A, Kirchner $G I$, et al. Bile duct damage after cold storage of deceased donor livers predicts biliary complications after liver transplantation. J Hepatol. 2013;58(6):1133-9.

27. Leonhardt S, Veltzke-Schlieker W, Adler A, Schott E, Hetzer R, Schaffartzik W, et al. Trigger mechanisms of secondary sclerosing cholangitis in critically ill patients. Critical Care. 2015;19:131. 


\section{Chapter 6}

Machine perfusion of the human liver: A systematic review

J. Reiling MC de Jong CHC Dejong J Fawcett

Submitted 


\section{Abstract}

\section{Background}

Extended criteria donors (ECD) livers have an increased susceptibility to cold storage associated preservation injury and the application of machine perfusion is therefore being explored. The purpose if this systematic review is to provide an overview of the safety and feasibility of hepatic machine perfusion (MP) techniques, and report the available data relating to clinical effectiveness.

\section{Methods}

A systematic search of the literature was conducted to identify studies that described hypothermic (HMP), sub- or mid-thermic (MMP/SMP) or normothermic machine perfusion (NMP) of human ECD livers.

\section{Key findings}

In total, 22 studies were identified: nine described HMP, nine described NMP and four described MMP. None of the centres encountered major technical difficulties resulting in the loss of a liver graft. Seven reports described the use of MP in a clinical transplant setting, all of which showed comparable or improved graft outcome compared to matched controls.

\section{Conclusions}

This review has shown that there is a wide variety of MP protocols currently being investigated. The technique is considered safe and feasible and small case series have shown promising results with regards to early graft function and biliary complication rates. 


\section{Introduction}

In recent years, there has been a renewed interest in the use of machine perfusion techniques in organ transplantation. Alexis Carrel and Charles Lindbergh pioneered the application of machine perfusion in the 1930s at the Rockefeller Institute in New York. The French surgeon and the great aviator built the first normothermic perfusion machine together in 1935 , which they used to study whole organs ex vivo. ${ }^{1}$ It would take another three decades for such a machine to facilitate the first successful liver transplant by Starzl. ${ }^{2,3}$ The definition of criteria for brain death and the development of preservation solutions such as University of Wisconsin solution lead to abandonment of the more complex and time consuming technique of machine perfusion and static cold storage methods were implemented.

The current discrepancy between organ supply and demand has in recent years lead to the use of organs from older and obese donors as well as those donating after circulatory death (DCD) for transplantation. ${ }^{4,5}$ Unfortunately livers procured from these extended criteria donors (ECD) are more susceptible to cold storage associated preservation injury and their use has been associated with inferior graft and recipient survival. ${ }^{6,7}$ In addition to a slightly increased thread of primary non-function, DCD liver transplant recipients are prone to develop ischaemic type biliary strictures (ITBS) with 9-31\% of recipients being affected. ${ }^{8,9}$ As a result, the likelihood of an offered DCD liver being turned down for transplantation in the United States increased four-fold in recent years. $^{10}$

Ex-situ machine perfusion techniques have been extensively studied in animal models and have been shown to be able to preserve or even improve graft function of extended criteria livers for long periods of time. ${ }^{11-13}$ Three benefits may quickly result from the introduction of these technologies: improved logistics (including long distance transport of donor organs), better assessment of marginal graft viability ${ }^{14}$ and the therapeutic intervention to improve graft function. ${ }^{15}$

Currently there are several (hepatic) perfusion techniques, largely distinguished by the temperature at which perfusion occurs. ${ }^{14}$ The purpose if this systematic review was to provide an overview of the safety and feasibility of machine perfusion techniques, and report the available data relating to clinical effectiveness.

\section{Methods}

\section{Search and study selection}

A systematic search of the literature was conducted using the Pubmed, Embase, Cochrane Library and Medline (Web of Science) databases in accordance with a preestablished PRISMA-P (Preferred Reporting Items for Systematic Reviews and Meta- 
Analysis - for Protocols) systematic review protocol (suppl. Data). ${ }^{15}$ No language or time restrictions were applied and the last search was conducted on the $18^{\text {th }}$ of March 2016. The following search strategy was employed: (("Liver Transplantation"[Mesh]) OR Liver Transplantation)) AND ((()(((normothermic perfusion) OR subnormothermic perfusion) OR hypothermic perfusion) OR hypothermic machine perfusion) OR subnormothermic machine perfusion) OR normothermic machine perfusion) $O R$ machine perfusion). To ensure all relevant papers were included, the reference lists of eligible articles as well as recently published reviews in the field were assessed.

\section{Eligibility criteria}

All studies using any type of ex-vivo machine perfusion technique on livers from human DCD (Maastricht category III) or marginal Donation after Brain Death (DBD) donors (e.g. severe steatosis and older donors) were included. Publications in languages other than English, Dutch, German or French were excluded. As the expected number of articles was small, all study types apart from reviews, conference papers and abstracts were included.

\section{Study selection and data extraction process}

Studies identified through the electronic database search were imported in a reference manager system and duplicates were removed. Two reviewers (JR, MCJ) independently screened the title, and when necessary the abstract, to identify potentially eligible manuscripts. Following the initial screen, the full text articles were independently reviewed to determine final inclusion. In case of disagreement between the two reviewers with regards to inclusion of a paper, a discussion took place and if necessary a third reviewer (CD) acted as an arbitrator. Data extraction was performed using a standardised data extraction sheet. Study design as well as donor demographics such as age, gender, cause of death, marginal donor criteria and warm and cold ischaemic time were recorded. In addition, the perfusion parameters from each circuit including perfusion temperature, type of perfusion machine, pump type and perfusate solution, single vessel or dual perfusion, perfusion flow and pressures as well as timing of perfusion (according to Karangwa et $a .^{14}$ ) were documented. Any tests performed to assess liver function prior to transplantation were noted and the authors were approached in case certain parameters were not provided in the manuscript.

\section{Outcome measures}

As machine perfusion of human donor livers is a relatively new technique, the primary endpoints were safety, feasibility and applicability of the perfusion protocol. The secondary outcomes only applied to machine perfusion protocols used in a transplant 
setting and included 6 month/1-year graft and patient survival, primary non-function and early allograft dysfunction rate as well as the development of hepatic artery thrombosis or any type of biliary complication (e.g. bile leak or ITBS).

\section{Data synthesis and quality assessment}

Only a qualitative summary of the included studies was performed due to the great heterogeneity of the included studies. Results are presented in tables as well as discussed in the text. Two reviewers (JR and MCJ) independently assessed the quality of the included case-control and cohort studies using the Newcastle-Ottawa Scale (NOS) and the final score was averaged.

\section{Results}

The combined search of the electronical databases identified 765 original manuscripts (Figure 6.1).

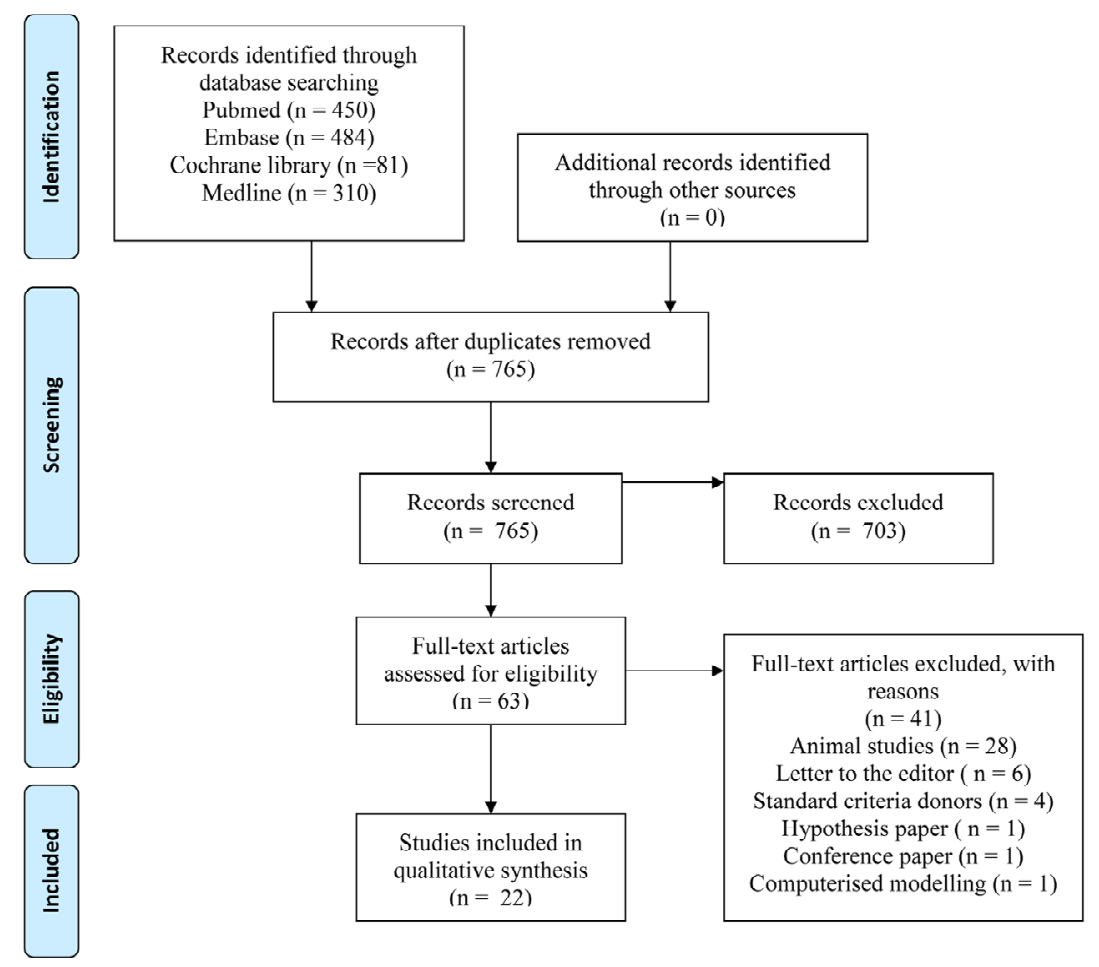

Figure 6.1 PRISMA flowchart for study selection. 
Following the initial screen, the full text of 63 articles was reviewed. Of those, 41 articles did not meet the inclusion criteria as they described animal experiments $(n=28)$, were written in response to previously published papers $(n=6)$, described standard criteria donors $(n=4)$, used human liver perfusion for computerised modelling $(n=1)$, or were conference or hypothesis papers $(n=2)$. Ultimately 22 papers were included in the systematic review; all but one were published within the last 5 years. In total, three case reports and fourteen manuscripts describing pre-clinical series were included. Only four transplant centres compared the results of their clinical series with matched controls. These studies were of high quality with NOS scores of 7 or greater.

\section{Machine perfusion protocols}

Table 6.1 shows the donor characteristics as well as perfusion parameters of the included studies. There were major differences in applied perfusion protocols with regards to temperature, device, perfusate and the use of oxygen. Overall, 89 livers were perfused and subsequently transplanted. From the 22 included manuscripts, nine described hypothermic machine perfusion protocols, all following a period of static cold storage. ${ }^{13,16-23}$ Each centre used a different device with Kidney Preservation Solution (KPS-1) solution being used as perfusate in the majority of cases. The biggest differences between the perfusion protocols were the supply of oxygen and dual versus portal vein only perfusion. Guarrera et al. pioneered HMP in marginal DBD livers by employing dual perfusion through both the hepatic artery and portal vein without active oxygenation. ${ }^{13,18}$ Similar protocols were used in two other pre-clinical series. ${ }^{19,20}$ Dutkowski and Schlegel et al. in Zurich on the other hand perfused DCD donors via the portal vein only with oxygenated KPS-1 solution. ${ }^{16,17,23}$ Dual oxygenated HMP through both the hepatic artery and portal vein was further explored by Westerkamp et al.. ${ }^{22}$ Across all HMP protocols, portal pressure was between 3-7 $\mathrm{mmHg}$ and hepatic artery pressures between 20 and $30 \mathrm{mmHg}$.

Two centres have described the application of mid-thermic machine perfusion. ${ }^{24-27}$ Bruinsma et al. in Boston perfused both discarded marginal DBD and DCD donor livers $(n=22)$ at $21^{\circ} \mathrm{C}$ using Williams' E solution. Livers were brought to their centre using static cold storage (SCS) and then perfused for three hours via both the hepatic artery and portal vein. In the end none were subsequently transplanted. Gradual oxygenated rewarming was applied by Hoyer et al. to livers offered via rescue allocation mechanisms. ${ }^{27}$ These livers were perfused for 1.5 hours to reach a final temperature of $20^{\circ} \mathrm{C}$ after which they were successfully transplanted. Between the two perfusion protocols, ${ }^{24,27}$ flow rates and pressures measured in the portal and arterial system were comparable. 


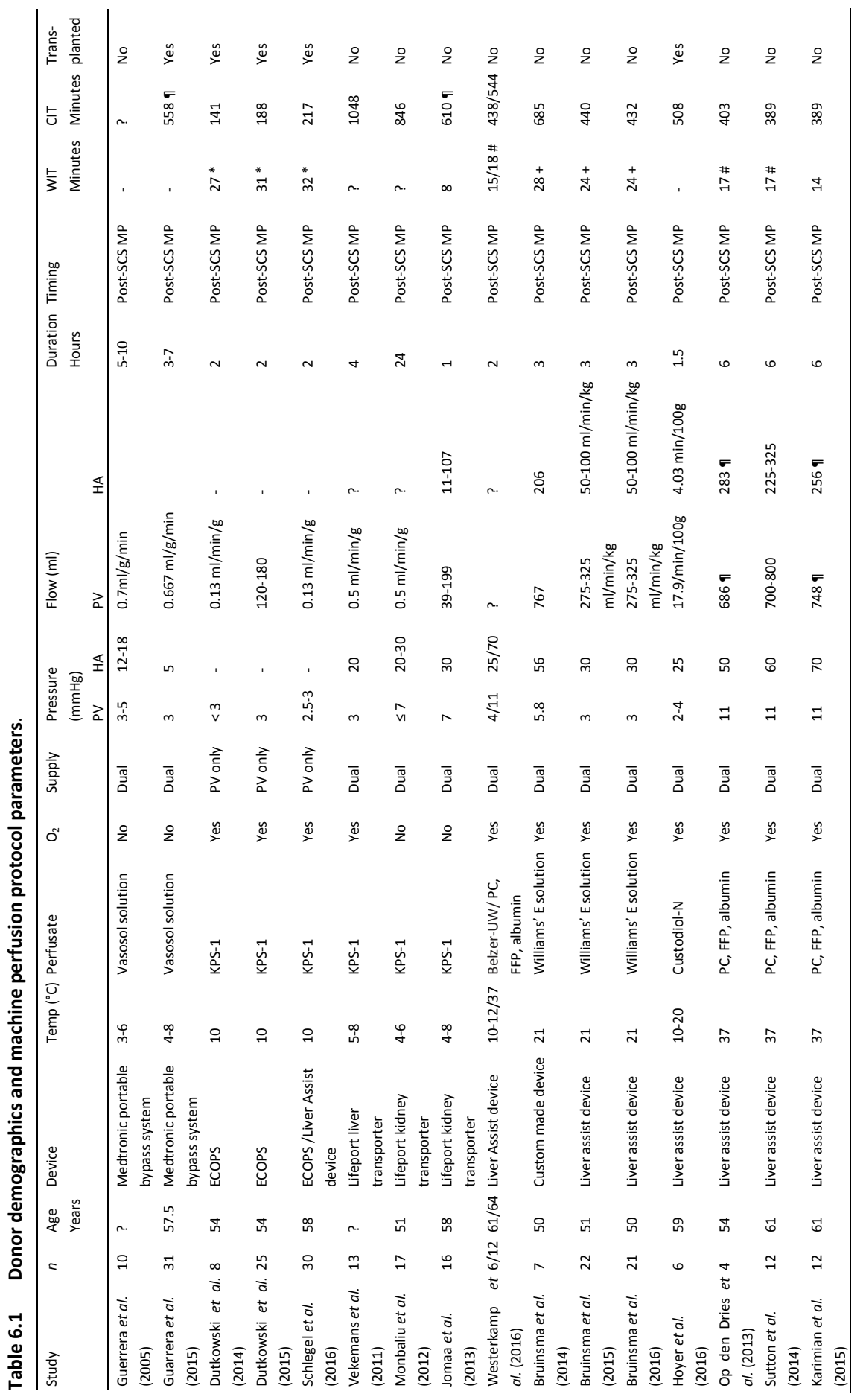




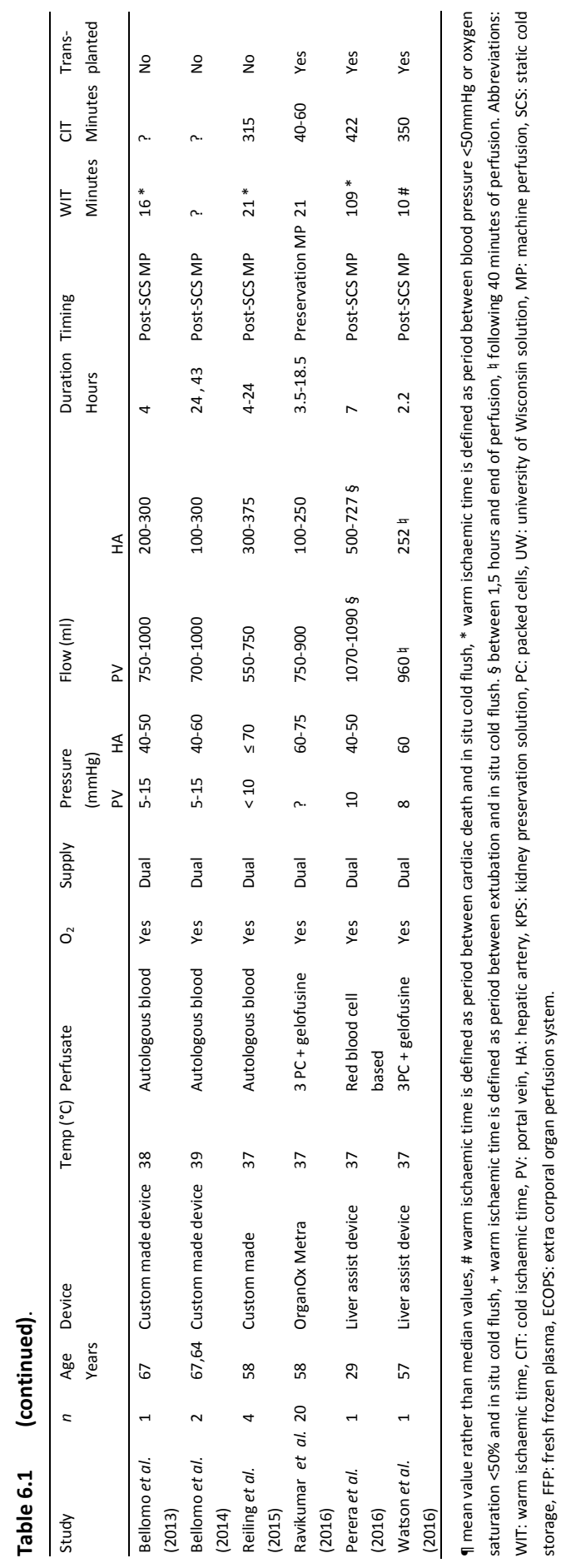


Normothermic machine perfusion was studied in six centres in both preclinical and clinical settings. ${ }^{28-36}$ This technique has been first studied by Op den Dries et al. in 2013 and was later continued by Sutton et al. in Groningen, the Netherlands. ${ }^{30,34}$ By using the Liver Assist device (Organ Assist, Groningen, the Netherlands), they perfused 12 discarded DBD and DCD livers with a mixture of packed cells, fresh frozen plasma and albumin. Normothermic perfusion in a pre-clinical setting was further studied by two centres in Australia using custom made devices. ${ }^{28,29,33}$ The first clinical results came from Perera et al. and Watson et al. in 2016. ${ }^{31,35}$ Using the same Liver Assist device, they each perfused a liver from a DCD donor, which was later successfully transplanted. The NMP device described and subsequently commercially developed in Oxford (OrganOx Metra ${ }^{\circledR}$, OrganOx Ltd, Oxford, United Kingdom) has been used in early clinical studies ${ }^{32}$; a much more substantial randomised controlled trial recently finished recruitment (ISRCTN39731134). All NMP protocols used blood-based perfusate, and pressures were limited between $5-15 \mathrm{mmHg}$ in the portal vein and 40-75 $\mathrm{mmHg}$ in the hepatic artery. Bellomo et al. reported the longest perfusion period (43 hours), however, this liver was not subsequently transplanted.

\section{Outcomes: Technical Feasibility}

All 22 studies showed that it is technically feasible to use machine perfusion of the liver. Only three papers included in this systematic review specifically mentioned the safety of the perfusion protocol. ${ }^{18,27,28}$ Therefore, all other authors were approached by email to comment and results are shown in Table 6.2. None of the centres encountered major technical difficulties resulting in the loss of a liver graft or exclusion from the study. Jomaa et al. reported a breach of sterility due to spillage from an external cooler. ${ }^{19}$ The recommended duration of use of the oxygenator in the Liver Assist device was only up to 6 hours. Perera et al. perfused the liver slightly longer, and the $\mathrm{pO}_{2}$ levels dropped towards the end of the perfusion. ${ }^{32}$ According to the authors, this did not threaten the quality of the graft. As the circuit used by Bellomo et al. as well as the circuit used in our centre in Brisbane were closed circuits using heparinised autologous blood, a scavenger system was applied to perfuse back leaked blood. ${ }^{29,30,33}$ Lastly, an airlock in the fluid sensing system of the OrganOx Metra $^{\circledR}$ resulted in a brief stop during transport and adjustments were made to the circuit to prevent this from happening in the future. ${ }^{28}$

All authors were asked to comment on transportability of the perfusion device used in their studies. The customised perfusion setups as well as the ECOPS or Liver Assist device are currently not transportable. Currently, only the OrganOx Metra ${ }^{\circledast}$ is suitable for transportation via road or air. A fully portable liver HMP device has been developed by LifePort (LifePort Liver Transporter, Organ Recovery systems, Chicago, USA) based on the prototype used by Guerrera et al.. This device is currently awaiting regulatory approval. 
As can be seen in the last column of Table 6.2, viability assessment was explored during NMP and controlled rewarming with bile production as well as lactate clearance being the points of focus.

Table 6.2 Safety and feasibility of machine perfusion techniques.

\begin{tabular}{|c|c|c|c|c|c|}
\hline Study & $n$ & $\begin{array}{l}\text { Technical } \\
\text { difficulties }\end{array}$ & $\begin{array}{l}\text { Graft } \\
\text { loss }\end{array}$ & $\begin{array}{l}\text { Transportabil } \\
\text { ity }\end{array}$ & Viability assessment \\
\hline Guarrera et al. $(2005,2015)$ & 41 & No & No & No & No \\
\hline $\begin{array}{l}\text { Dutkowski et al. (2015), } \\
\text { Schlegel et al. (2016) }\end{array}$ & 30 & No & No & No & No \\
\hline Vekemans et al. (2011) & 13 & No & No & No & No \\
\hline Monbaliu et al. (2012) & 17 & No & No & No & No \\
\hline Jomaa et al. (2013) & 16 & $\begin{array}{l}\text { Yes, sterility } \\
\text { breach }\end{array}$ & No & No & No \\
\hline $\begin{array}{l}\text { Westerkamp et al. (2016), } \\
\text { Op den Dries et al. (2013), } \\
\text { Sutton at al. (2014), } \\
\text { Karimian et al. (2015) }\end{array}$ & $30^{*}$ & No & No & No & $\begin{array}{l}\text { Yes, cumulative bile } \\
\text { production } \geq 10 \text { g after } 2,5 \\
\text { hours and } \geq 4 \text { g bile } \\
\text { production between } 1,5 \text { and } \\
2,5 \text { hours }\end{array}$ \\
\hline $\begin{array}{l}\text { Bruinsma et al. }(2014,2015 \text {, } \\
\text { 2016) }\end{array}$ & 22 & No & No & No & No \\
\hline Hoyer et al. (2016) & 6 & No & No & $\begin{array}{l}\text { Not } \\
\text { applicable }\end{array}$ & $\begin{array}{l}\text { Delta glucose ( } 90 \text { minutes - } \\
60 \text { minutes) }\end{array}$ \\
\hline Bellomo et al. $(2013,2014)$ & 3 & Yes, bleeding & No & No & No \\
\hline Reiling et al. (2015) & 4 & Yes, bleeding & No & No & No \\
\hline Ravikumar et al. (2016) & 20 & $\begin{array}{l}\text { Yes, airlock in } \\
\text { fluid sensing } \\
\text { system }\end{array}$ & No & Yes & No \\
\hline Perera et al. (2016) & 1 & Yes, oxygenator & No & No & $\begin{array}{l}\text { Lactate }<2 \mathrm{mmol} / \mathrm{L} \\
<2 \mathrm{~h}+\text { bile production }\end{array}$ \\
\hline Watson et al. (2016) & 1 & No & No & No & $\begin{array}{l}\text { Lactate clearance, } \\
\text { macroscopic appearance, } \\
\text { flow }\end{array}$ \\
\hline
\end{tabular}

* It was unclear if an overlap existed between the normothermic perfused livers described in Sutton et al. 2014 and Westerkamp et al. 2016.

\section{Outcomes: Complication Rate and Survival}

Seven reports have described the use of machine perfusion in a clinical transplant setting of which four were control matched case series (Table 6.3). Primary nonfunction was defined according to Olthoff et al. in all case-control series. ${ }^{37}$ The definition of ITBS according to Buis et al. ${ }^{38}$ was only used in one report. 


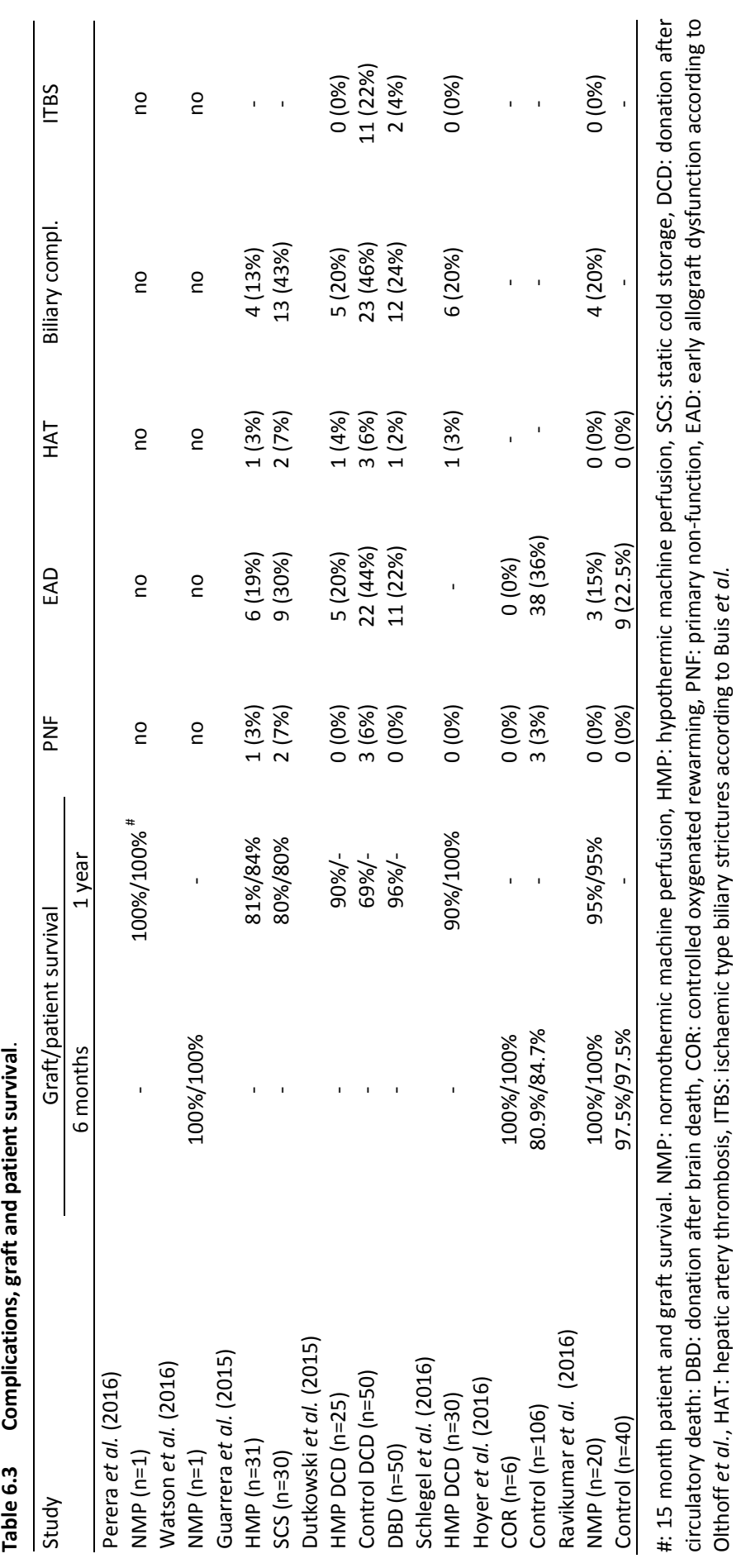


Cold perfused (HMP) livers, compared to static cold stored controls in a series of marginal DBD liver donors, showed comparable incidence of primary non-function and early allograft dysfunction with similar 1-year graft and patient survival. ${ }^{18}$ of the 31 perfused livers, significantly fewer patients developed biliary complications (13\% versus $43 \%$ of the controls), and more specifically less biliary stricture formation ( $10 \%$ versus $33 \%$ ) than static cold stored livers. It is unclear from the report which of the strictures were anastomotic or ITBS. Dutkowski et al. compared DCD livers subjected to oxygenated HMP with both non-perfused DCD livers and DBD matched controls. ${ }^{16}$ None of the perfused DCD livers developed primary non-function and the authors reported $50 \%$ less early allograft dysfunction compared to non-perfused DCD controls. Furthermore, none of the patients developed ITBS during a median follow-up of 448 days. Ultimately, one-year cholangiopathy-free graft survival of the HMP group was significantly higher than the cold stored DCD group and comparable to the DBD controls $(90 \%$ versus $69 \%$ and 96 In a later publication describing an additional 5 DCD HMP livers, no recipients of HMP perfused DCD livers had developed biliary strictures. $^{23}$

Hoyer at al. compared their 6 controlled oxygenated rewarming (COR) livers with a matched historical cohort of rescue offer control livers. ${ }^{27}$ All perfused livers had excellent graft function whereas in the control group, $3(2.8 \%)$ patients developed primary non-function and 38 (35.9\%) development early allograft dysfunction. The development of biliary complications was not reported in this small controlled rewarming study.

The two case reports ${ }^{34,35}$ reported a $100 \%$ 6-month and 15 -month graft survival as well as patient survival following NMP without the development of complications. ${ }^{32,35}$ The only study comparing NMP with matched cold stored livers thus far was reported by Ravikumar et al.. ${ }^{28}$ Compared to matched controls, the rate of EAD was similar in the NMP group with a 6-month graft and patient survival of $100 \%$. The rate of biliary complications in the control group was not given but in the NMP group, 4 patients who received a DBD graft developed biliary anastomotic strictures, and these were subsequently stented.

\section{Discussion}

This systematic review shows that machine perfusion of donor livers is an emerging area of clinical research. Within the last 5 years, 21 papers were published regarding its application in human liver transplantation and results of the first clinical series are being presented. There was great heterogeneity amongst the perfusion protocols with different perfusion temperatures and perfusion devices being used which makes direct comparisons difficult. 
Cold perfusion (HMP) was the first perfusion modality to be studied in the modern era of liver transplantation. The proposed working mechanism of this technique is the continuous circulation of perfusion solution (containing metabolic substrates and antioxidants) that supports liver metabolism whereas toxic waste products are washed out of the graft. Guarrera at al. first reported the use of this technique in DBD standard criteria donors in $2010 .^{39}$ Serum markers for liver injury as well as markers of ischaemia reperfusion injury and acute phase mediators were reduced following HMP. ${ }^{40,41}$ This was later confirmed in a larger series of marginal DBD livers, included in this review, where patient and graft survival was comparable to matched DBD controls. The simplicity of this perfusion method combined with the compact, portable nature of the perfusion system are attractive features of HMP. No oxygen carrier is needed with this system so that, in the event of pump failure, the organ remains in static cold storage.

Where dissolved oxygen seems sufficient for DBD livers, active oxygenation was found better for the preservation of DCD livers. ${ }^{42,43}$ Pre-clinical studies showed that supplementing oxygen during HMP lead to a rapid reduction of mitochondrial respiration rate after $60-90$ minutes of perfusion resulting in a marked increase in hepatic ATP content and reduction of cellular damage following reperfusion. ${ }^{42,44}$ In clinical studies, oxygenated HMP for only a short period of time prior to transplantation reportedly reduced the incidence of early allograft dysfunction by $50 \%{ }^{16}$ and a marked reduction in the development of ITBS was seen despite a longer median WIT than the control non-perfused DCD cohort. Since bile duct blood supply is principally arterial rather than portal, dual oxygenated HMP via both the hepatic artery and portal vein has been proposed. The application of this technique prevented the development of arteriolonecrosis of the peribiliary plexus in a porcine model, a risk factor associated with the development of ITBS. A randomised control trial, comparing dual oxygenated HMP with SCS control DCD liver grafts is currently underway to determine whether this technique leads to improved preservation of the bile ducts (Netherlands Trial Registry, NTR4493; www.trialregister.nl).

Machine perfusion under hypothermic conditions does not easily allow for assessment of graft function, but oxygenated normothermic perfusion brings liver metabolism up to physiological conditions and viability can be determined. On the other hand, the technique is more complex (oxygenation and nutrient supply are required) and pump failure renders the graft immediately susceptible to warm ischaemia, emphasising the need for (expensive) meticulous engineering of the devices. Extensive preclinical work has shown that NMP is able to ameliorate hepatic warm ischaemic injury, ${ }^{11,31,45}$ especially when livers are not first stored on ice prior to establishing NMP. ${ }^{46}$ Whereas HMP was only performed for short periods of time, NMP graft perfusion up to 18 hours has been achieved; this could significantly improve transplant logistics. ${ }^{28}$ Air transportation of a perfusion device has not yet been trialled but would be highly desirable. Despite the major achievements to date in the 
application of NMP, the complexity of liver metabolism means that optimal perfusion conditions (e.g. substrate, oxygenation, micronutrient and hormonal requirements, osmolality control, pulsatile perfusion) to preserve and improve steatotic or DCD grafts, in particular, are yet to be fully explored.

Lactate clearance below $2 \mathrm{mmol} / \mathrm{L}$ within 2 hours and bile production during perfusion have been proposed as criteria for viability assessment. ${ }^{32,34}$ Both are easy to assess during perfusion but larger trials are needed to determine if these factors accurately predict long term graft function. At this point, neither HMP nor NMP have addressed the assessment of biliary viability or integrity, currently the most important limiting factor in the use of DCD grafts. Both HMP and NMP have shown to preserve the peribiliary plexus and peribiliary glands in rat and porcine models, and HMP convincingly reduced the incidence of ITBS in perfused livers. ${ }^{47,48}$ Nevertheless, noninvasive methods are required to assess biliary integrity and predict biliary cholangiopathy. Currently histological assessment of common hepatic bile duct sections at the end of cold storage is the best available. ${ }^{49,50}$

One of the limitations of this review is that there are no published randomised control trials to date as the application of machine perfusion is only just entering clinical practice. Furthermore, the perfusion protocols are too heterogeneous to perform a meta-analysis. Investigators in the field of machine perfusion have proposed uniform reporting criteria to facilitate meta-analysis in the future. ${ }^{14}$ All clinical studies reported EAD according to Olthoff et al. but uniform definitions of WIT as well as ITBS are lacking. ${ }^{37}$ As with all new technology, technical feasibility and safety are important aspects. Unfortunately, only 3 out of the 22 papers elaborated on safety and loss of grafts due to technical difficulties. Machine perfusion is more complicated than static cold storage and detailed reporting is required to inform the transplant community of the risks involved. This is especially important for normothermic perfusion, as pump failure immediately leads to warm ischaemia, with likely graft loss. Encouragingly, it appeared from the information we received upon request, that none of the research groups encountered serious technical problems that lead to the loss of grafts.

The expansion of the donor pool using machine perfusion techniques will come at a cost. Not only are the commercially available perfusion devices expensive (e.g. OrganOx metra ${ }^{\circledR}, £ 125,000.00$ ), the cost of disposables (between $€ 4000$ and $£ 10000$ ), perfusion solutions, additives and staff to run the perfusion need to be taken into account. This should however be weighed against the reduction in patients dying on the waiting list while waiting for a life-saving graft. It is recognised that running a DCD program is more expensive than DBD programs. ${ }^{51}$ However, if machine perfusion results in a lower incidence of primary non-function, early allograft dysfunction and even ITBS, a reduction in the costs of DCD liver transplantation could be achieved.

In conclusion, this review has shown that there are a wide variety of machine perfusion protocols currently being investigated with hypothermic and normothermic perfusion, as well as controlled oxygenated rewarming, entering clinical trials. The 
techniques are technically feasible with no reported graft loss due to technical complications. The small case series published thus far have shown promising results with regards to early graft function and biliary complication rates. Results of large randomised trials are awaited to determine if machine perfusion is truly better than static cold storage. 


\section{References}

1. Carrel A, Lindbergh CA. The culture of whole organs. Science. 1935;81(2112):621-3.

2. Starzl TE, Groth CG, Brettschneider L, Penn I, Fulginiti VA, Moon JB, et al. Orthotopic homotransplantation of the human liver. Ann Surg. 1968;168(3):392-415.

3. Brettschneider L, Daloze PM, Huguet C, Porter KA, Groth CG, Kashiwagi N, et al. The use of combined preservation techniques for extended storage of orthotopic liver homografts. Surg Gynecol Obstet. 1968;126(2):263-74.

4. Durand F, Renz JF, Alkofer B, Burra P, Clavien PA, Porte RJ, et al. Report of the Paris consensus meeting on expanded criteria donors in liver transplantation. Liver Transpl. 2008;14(12):1694-707.

5. Bellingham JM, Santhanakrishnan C, Neidlinger N, Wai P, Kim J, Niederhaus S, et al. Donation after cardiac death: a 29-year experience. Surgery. 2011;150(4):692-702.

6. Jay CL, Lyuksemburg V, Ladner DP, Wang E, Caicedo JC, Holl JL, et al. Ischemic cholangiopathy after controlled donation after cardiac death liver transplantation: a meta-analysis. Ann Surg. 2011;253(2):259-64.

7. Feng S, Goodrich NP, Bragg-Gresham JL, Dykstra DM, Punch JD, DebRoy MA, et al. Characteristics associated with liver graft failure: the concept of a donor risk index. Am J Transplant. 2006;6(4): 783-90.

8. Dubbeld J, Hoekstra H, Farid W, Ringers J, Porte RJ, Metselaar HJ, et al. Similar liver transplantation survival with selected cardiac death donors and brain death donors. Br J Surg. 2010;97(5):744-53.

9. Laing RW, Scalera I, Isaac J, Mergental H, Mirza DF, Hodson J, et al. Liver transplantation using grafts from donors after circulatory death: A propensity-matched study from a single centre. Am J Transplant. 2016.

10. Orman ES, Barritt ASt, Wheeler SB, Hayashi PH. Declining liver utilization for transplantation in the United States and the impact of donation after cardiac death. Liver Transpl. 2013;19(1):59-68.

11. Butler AJ, Rees MA, Wight DG, Casey ND, Alexander G, White DJ, et al. Successful extracorporeal porcine liver perfusion for $72 \mathrm{hr}$. Transplantation. 2002;73(8):1212-8.

12. Vairetti M, Ferrigno A, Carlucci F, Tabucchi A, Rizzo V, Boncompagni E, et al. Subnormothermic machine perfusion protects steatotic livers against preservation injury: A potential for donor pool increase? Liver Transplant. 2009;15(1):20-9.

13. Guarrera JV, Estevez J, Boykin J, Boyce R, Rashid J, Sun S, et al. Hypothermic machine perfusion of liver grafts for transplantation: technical development in human discard and miniature swine models. Transplant Proc. 2005;37(1):323-5.

14. Karangwa SA, Dutkowski P, Fontes P, Friend PJ, Guarrera JV, Markmann JF, et al. Machine Perfusion of Donor Livers for Transplantation: A Proposal for Standardized Nomenclature and Reporting Guidelines. 2016.

15. Moher D, Shamseer L, Clarke M, Ghersi D, Liberati A, Petticrew M, et al. Preferred reporting items for systematic review and meta-analysis protocols (PRISMA-P) 2015 statement. Systematic reviews. 2015;4:1.

16. Dutkowski P, Polak WG, Muiesan P, Schlegel A, Verhoeven CJ, Scalera I, et al. First comparison of hypothermic oxygenated perfusion versus static cold storage of human donation after cardiac death liver transplants. Ann Surg. 2015;262(5):764-71.

17. Dutkowski P, Schlegel A, De Oliveira M, Müllhaupt B, Neff F, Clavien PA. HOPE for human liver grafts obtained from donors after cardiac death. J Hepatol. 2014;60(4):765-72.

18. Guarrera JV, Henry SD, Samstein B, Reznik E, Musat C, Lukose TI, et al. Hypothermic machine preservation facilitates successful transplantation of "orphan" extended criteria donor livers. Am J Transplant. 2015;15(1):161-9.

19. Jomaa A, Gurusamy K, Siriwardana PN, Claworthy I, Collier S, de Muylder P, et al. Does hypothermic machine perfusion of human donor livers affect risks of sinusoidal endothelial injury and microbial infection? A feasibility study assessing flow parameters, sterility, and sinusoidal endothelial ultrastructure. Transplant Proc. 2013;45(5):1677-83. 
20. Monbaliu D, Liu Q, Libbrecht L, De Vos R, Vekemans K, Debbaut C, et al. Preserving the morphology and evaluating the quality of liver grafts by hypothermic machine perfusion: a proof-of-concept study using discarded human livers. Liver Transplant. 2012;18(12):1495-507.

21. Vekemans K, van Pelt J, Komuta M, Wylin T, Heedfeld V, Detry O, et al. Attempt to rescue discarded human liver grafts by end ischemic hypothermic oxygenated machine perfusion. Transplant Proc. 2011;43(9):3455-9.

22. Westerkamp AC, Karimian N, Matton APM, Mahboub P, van Rijn R, Wiersema-Buist J, et al. Oxygenated hypothermic machine perfusion after static cold storage improves hepatobiliary function of extended criteria donor livers. Transplantation. 2016;100(4):825-35.

23. Schlegel A, Kron P, De Oliveira ML, Clavien PA, Dutkowski P. Is single portal vein approach sufficient for hypothermic machine perfusion of DCD liver grafts? J Hepatol. 2016;64(1):239-41.

24. Bruinsma BG, Avruch JH, Weeder PD, Sridharan GV, Uygun BE, Karimian NG, et al. Functional human liver preservation and recovery by means of subnormothermic machine perfusion. J Vis Exp. 2015(98).

25. Bruinsma BG, Sridharan GV, Weeder PD, Avruch JH, Saeidi N, Ozer S, et al. Metabolic profiling during ex vivo machine perfusion of the human liver. Sci Rep. 2016;6:22415.

26. Bruinsma BG, Yeh H, Özer S, Martins PN, Farmer A, Wu W, et al. Subnormothermic machine perfusion for ex vivo preservation and recovery of the human liver for transplantation. Am J Transplant. 2014;14(6):1400-9.

27. Hoyer DP, Mathe Z, Gallinat A, Canbay AC, Treckmann JW, Rauen U, et al. Controlled Oxygenated Rewarming of Cold Stored Livers Prior to Transplantation: First Clinical Application of a New Concept. Transplantation. 2016;100(1):147-52.

28. Ravikumar R, Jassem W, Mergental H, Heaton N, Mirza D, Perera MT, et al. Liver transplantation after ex vivo normothermic machine preservation: a Phase 1 (first-in-man) clinical trial. Am J Transplant. 2016;16(6):1779-87.

29. Bellomo R, Marino B, Starkey G, Wang BZ, Fink MA, Zhu N, et al. Normothermic extracorporeal human liver perfusion following donation after cardiac death. Crit Care Resusc. 2013;15(2):78-82.

30. Bellomo R, Marino B, Starkey G, Fink M, Wang BZ, Eastwood GM, et al. Extended normothermic extracorporeal perfusion of isolated human liver after warm ischaemia: a preliminary report. Criti Care Resusc. 2014;16(3):197-201.

31. op den Dries S, Karimian N, Sutton ME, Westerkamp AC, Nijsten MW, Gouw AS, et al. Ex vivo normothermic machine perfusion and viability testing of discarded human donor livers. Am J Transplant. 2013;13(5):1327-35

32. Perera T, Mergental H, Stephenson B, Roll GR, Cilliers $H$, Liang $R$, et al. First human liver transplantation using a marginal allograft resuscitated by normothermic machine perfusion. Liver Transpl. 2016;22(1):120-4.

33. Reiling J, Lockwood DS, Simpson AH, Campbell CM, Bridle KR, Santrampurwala N, et al. Urea production during normothermic machine perfusion: Price of success? Liver Transpl. 2015;21(5): 700-3.

34. Sutton ME, Op Den Dries S, Karimian N, De Boer MT, Wiersema-Buist J, Gouw ASH, et al. Criteria for viability assessment of discarded human donor livers during ex-vivo normothermic machine perfusion. Liver Transplant. 2014;20:S240.

35. Watson CJE, Kosmoliaptsis V, Randle LV, Russell NK, Griffiths WJH, Davies S, et al. Preimplant Normothermic Liver Perfusion of a Suboptimal Liver Donated After Circulatory Death. Am J Transplant. 2016;16(1):353-7.

36. Karimian N, Matton AP, Westerkamp AC, Burlage LC, Op den Dries S, Leuvenink HG, et al. Ex Situ Normothermic Machine Perfusion of Donor Livers. J Vis Exp. 2015(99):e52688.

37. Olthoff KM, Kulik L, Samstein B, Kaminski M, Abecassis M, Emond J, et al. Validation of a current definition of early allograft dysfunction in liver transplant recipients and analysis of risk factors. Liver Transpl. 2010;16(8):943-9.

38. Buis $\mathrm{Cl}$, Verdonk RC, Van der Jagt EJ, van der Hilst CS, Slooff MJ, Haagsma EB, et al. Nonanastomotic biliary strictures after liver transplantation, part 1: Radiological features and risk factors for early vs. late presentation. Liver Transpl. 2007;13(5):708-18. 
39. Guarrera JV, Henry SD, Samstein B, Odeh-Ramadan R, Kinkhabwala M, Goldstein MJ, et al. Hypothermic machine preservation in human liver transplantation: the first clinical series. Am J Transplant. 2010;10(2):372-81.

40. Henry SD, Nachber E, Tulipan J, Stone J, Bae C, Reznik L, et al. Hypothermic machine preservation reduces molecular markers of ischemia/reperfusion injury in human liver transplantation. Am J Transplant. 2012;12(9):2477-86.

41. Tulipan JE, Stone J, Samstein B, Kato T, Emond JC, Henry SD, et al. Molecular expression of acute phase mediators is attenuated by machine preservation in human liver transplantation: preliminary analysis of effluent, serum, and liver biopsies. Surgery. 2011;150(2):352-60.

42. Dutkowski P, Furrer K, Tian Y, Graf R, Clavien PA. Novel short-term hypothermic oxygenated perfusion (HOPE) system prevents injury in rat liver graft from non-heart beating donor. Ann Surg. 2006; 244(6):968-76; discussion 76-7.

43. t Hart NA, van der Plaats A, Faber A, Leuvenink HGD, Olinga P, Wiersema-Buist J, et al. Oxygenation during hypothermic rat liver preservation: an in vitro slice study to demonstrate beneficial or toxic oxygenation effects. Liver Transplant. 2005;11(11):1403-11.

44. Schlegel A, Rougemont OD, Graf R, Clavien PA, Dutkowski P. Protective mechanisms of end-ischemic cold machine perfusion in DCD liver grafts. J Hepatol. 2013;58(2):278-86.

45. St Peter SD, Imber CJ, Lopez I, Hughes D, Friend PJ. Extended preservation of non-heart-beating donor livers with normothermic machine perfusion. Br J Surg. 2002;89(5):609-16.

46. Reddy SP, Bhattacharjya S, Maniakin N, Greenwood J, Guerreiro D, Hughes D, et al. Preservation of porcine non-heart-beating donor livers by sequential cold storage and warm perfusion. Transplantation. 2004;77(9):1328-32.

47. Liu Q, Nassar A, Farias K, Buccini L, Baldwin W, Mangino M, et al. Sanguineous normothermic machine perfusion improves hemodynamics and biliary epithelial regeneration in donation after cardiac death porcine livers. Liver Transplant. 2014;20(8):987-99.

48. Op den Dries S, Sutton ME, Karimian N, de Boer MT, Wiersema-Buist J, Gouw ASH, et al. Hypothermic oxygenated machine perfusion prevents arteriolonecrosis of the peribiliary plexus in pig livers donated after circulatory death. PloS One. 2014;9(2):e88521.

49. op den Dries S, Westerkamp AC, Karimian N, Gouw AS, Bruinsma BG, Markmann JF, et al. Injury to peribiliary glands and vascular plexus before liver transplantation predicts formation of nonanastomotic biliary strictures. J Hepatol. 2014;60(6):1172-9.

50. Brunner SM, Junger H, Ruemmele P, Schnitzbauer AA, Doenecke A, Kirchner GI, et al. Bile duct damage after cold storage of deceased donor livers predicts biliary complications after liver transplantation. J Hepatol. 2013;58(6):1133-9.

51. Singhal A, Wima K, Hoehn RS, Quillin RC, 3rd, Woodle ES, Paquette IM, et al. Hospital Resource Use with Donation after Cardiac Death Allografts in Liver Transplantation: A Matched Controlled Analysis from 2007 to 2011. J Am Coll Surg. 2015;220(5):951-8. 


\section{Chapter 7 \\ Urea production during Normothermic Machine \\ Perfusion- Price of Success?}

J Reiling

DSR Lockwood

AH Simpson

CM Campbell

KR Bridle

N Santrampurwala

L Britton

DHG Crawford

CHC Dejong

J Fawcett

Liver Transplantation, 2015;21(5):700-3 


\section{Abstract}

\section{Background}

Normothermic machine perfusion (NMP) holds great promise as an ex-vivo organ maintenance system but many facets of liver physiology need to be considered in its establishment. We have successfully performed ex vivo normothermic oxygenated perfusion in 4 human donor livers rejected as unsuitable for transplantation. This manuscript describes one such perfusion where hyper osmolality in the perfusate from urea production and hyperglycaemia highlights the complexity of organ maintenance in NMP systems.

\section{Method}

Organ retrieval was performed in a standard fashion using University of Wisconsin solution. In addition, blood from the donor was collected and used as NMP perfusate. In the circuit, perfusate draining from the IVC was driven through a single centrifugal pump, then onwards through an oxygenator / heat exchanger before being split into a pressure-controlled hepatic artery supply and gravity fed portal venous supply via a reservoir. Throughout the perfusion period of twenty-four hours there was continuous monitoring of haemodynamic parameters and blood, bile, liver and bile duct tissue samples were collected.

\section{Key findings}

Acid-base homeostasis was restored quickly and, even though there was a substantial initial transaminase release, thereafter little ongoing enzyme flux was observed. Perfusate urea concentration steadily rose to a final concentration of $103 \mathrm{mmol} / \mathrm{L}$. In conjunction with the electrolyte and glucose measurements, this contributed greatly to a steady increase in calculated serum osmolality from $342 \mathrm{mmol} / \mathrm{L}$ to $414 \mathrm{mmol} / \mathrm{L}$ over time.

\section{Conclusions}

We believe that it is important to maintain physiological parameters, including osmolality, in NMP systems as near normal as possible. Provision for dialysis of the perfusate during NMP may be necessary especially for long duration of perfusion. 


\section{To the editors}

Normothermic oxygenated Machine Perfusion (NMP) has been proposed as a technique that may provide the means to preserve organ function but moreover accurately predict clinical outcome because graft function can be analysed pretransplant. Promising results have been obtained in several animal models and a recent pilot study in discarded human donor livers showed that normothermic machine perfusion was feasible and graft viability could be assessed. ${ }^{1}$

We have successfully established an ex vivo normothermic oxygenated perfusion circuit using only a single centrifugal pump. We have perfused 4 human donor livers, currently deemed unsuitable for transplantation by local criteria. Interestingly, we observed high levels of calculated osmolality in the perfusate; a problem which we believe should be addressed in order to implement this new technique in clinical practice.

\section{Methods}

\section{Organ retrieval}

The human donor livers used in this report were deemed unsuitable for transplant based on current local criteria. Three livers were rejected due to their age as a donation after cardiac death (DCD) donor and one, retrieved from a brain dead (DBD) donor, due to the presence of steatohepatitis. Organ retrieval and cold perfusion were undertaken according to local protocol using heparinised saline followed by University of Wisconsin solution, with priority given at all times to organs destined for clinical transplantation. In accordance with local legislation, no heparin was administrated to DCD donors prior to organ retrieval. Donor blood was collected from the right atrium and later used to prime the perfusion circuit. Both the liver and the collected blood were stored on ice until the normothermic liver perfusion was commenced. The mean total period of cold ischaemia prior to perfusion was 5 hours and 42 minutes ( \pm 2 hours and 45 minutes).

Approval was obtained from the Metro South Human Research Ethics Committee and the University of Queensland to perform the below described study protocol.

\section{Perfusion circuit}

The perfusion circuit was designed in our centre and consisted of a single centrifugal pump (ROTAFLOW; Maquet, Rastatt, Germany) to drive pressure controlled hepatic artery perfusion and maintain filling of a reservoir for a gravity fed portal venous supply (Figure 7.1). Other circuit components included an oxygenator (Dideco TM Kids 
D101; Sorin Group, Mirandola, Italy) and a heat exchanger set at $37{ }^{\circ} \mathrm{C}$. A scavenger system with a roller pump returned leaked perfusate back to the portal reservoir.

The circuit was primed with $4 \%$ albumin solution and bubbles were removed. Blood was then slowly added and bicarbonate was administered to correct acidosis. When a citrate-based anticoagulation solution was used during donor blood retrieval, calcium gluconate was also added to normalise calcium levels. A minimum circuit volume of $1.5 \mathrm{~L}$ was used with a haematocrit of at least $20 \%$.

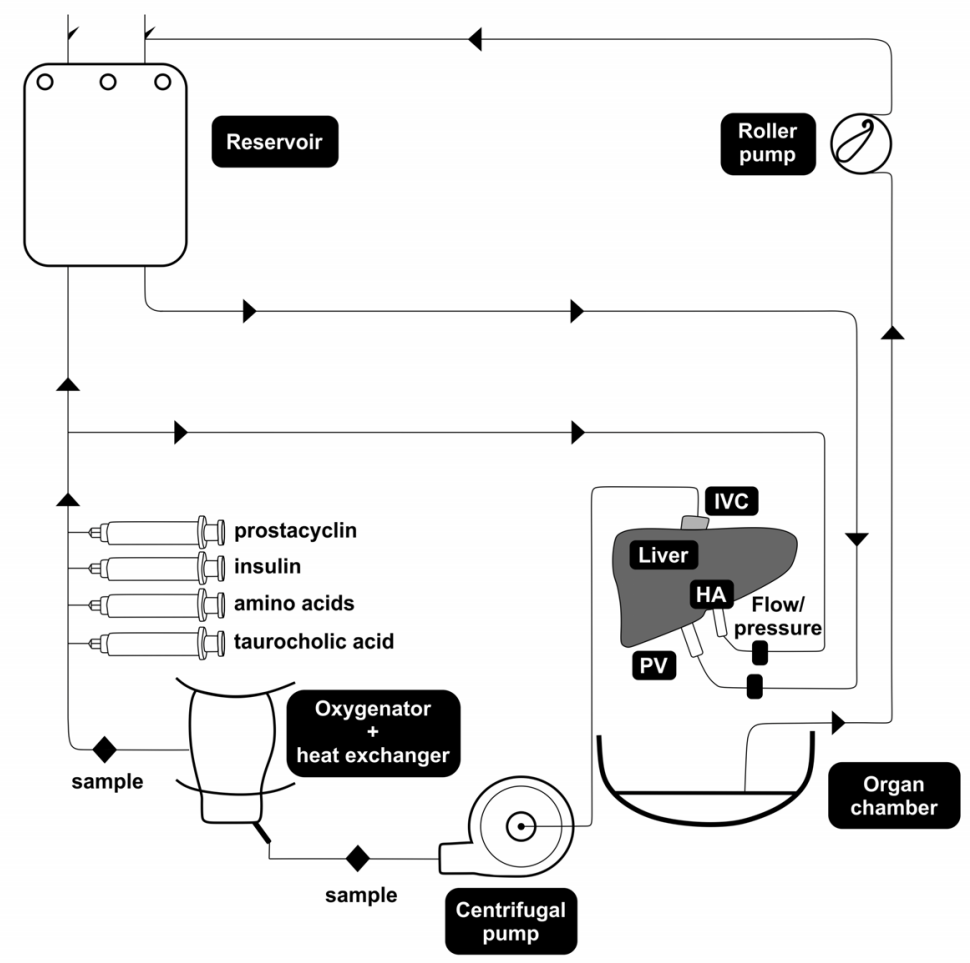

Figure 7.1 Schematic overview of the perfusion circuit. The liver is placed in the organ chamber and connected to the centrifugal pump which directly supplies the hepatic artery. The portal vein is gravity fed via the reservoir. A roller pump circulates leaked perfusate back into the reservoir.

\section{Liver preparation and commencement of normothermic perfusion}

A routine back table procedure was performed and the bile duct was cannulated with an infant feeding catheter for bile collection. The top end of the vena cava was closed and a 34F cannula (Medtronic; Minneapolis, MN, USA) was inserted in the inferior vena cava. 
The common hepatic artery was cannulated with a $16 \mathrm{~F}$ cannula (Medtronic; Minneapolis, MN, USA) and a portal venous cannula (Maquet; Rastatt, Germany), inserted during organ retrieval, was left in situ. Before connecting the liver to the circuit, it was flushed with $2 \mathrm{~L}$ cold saline to wash out residual UW solution.

The pump was started at $1.4 \mathrm{~L} /$ minute and adjusted to limit the hepatic artery pressure to $70 \mathrm{mmHg}$ and flows were confirmed using dedicated Doppler probes (Medistim; Oslo, Norway) on the hepatic artery (300-375 mL/minute) and portal vein (550 - $750 \mathrm{~mL} /$ minute). Pressures were measured in the hepatic artery and portal vein with a dual pressure monitoring kit (Edwards Lifesciences; Irvine, CA, USA). Throughout the perfusion period there was continuous monitoring of hemodynamic parameters and blood, bile, liver and bile duct tissue samples were collected.

\section{Perfusate additives}

Taurocholic acid (7 mL/hour), prostacyclin ( $8 \mathrm{mcg} /$ hour) and insulin (50-200 IU/hour) were continuously infused and heparin was administered as a bolus according to the activated clotting time. In addition, amino acids $(20 \mathrm{~mL} /$ hour $)$ were continuously infused during the latter two perfusions as has been reported in other systems. ${ }^{1}$

\section{Results}

The four livers included in this study were perfused for between 3 and 24 hours. Serial perfusate samples were taken and used for biochemical analysis (Figure 7.2A). During the perfusion the livers showed an initial large release of transaminases followed by steady decline over time. Lactate levels in the circuit rapidly normalised in all four cases to less than $1.5 \mathrm{mmol} / \mathrm{L}$ following a mean peak of $7.12 \pm 1.76 \mathrm{mmol} / \mathrm{L}$ (data not shown). All four livers produced bile during the perfusion period (data not shown).

Perfusate urea concentration steadily increased over the perfusion period; in the fourth NMP (NMP4), perfused for 24 hours because graft performance was very good based on appearance, metabolic parameters and bile production, the final concentration exceeded $100 \mathrm{mmol} / \mathrm{L}$ (Figure 7.2B). Glucose concentration was also noted to be high during perfusion, which was unresponsive to the administration of large doses of insulin (>50 units/hour) into the circuit (Figure 7.2C). In conjunction with the electrolyte measurements, this meant that, in all four liver perfusions, the calculated serum osmolality was over the upper limit of normal (normal range 275-295 mmol/L); in the case of NMP4 this was quite markedly so (Figure 7.2D). 

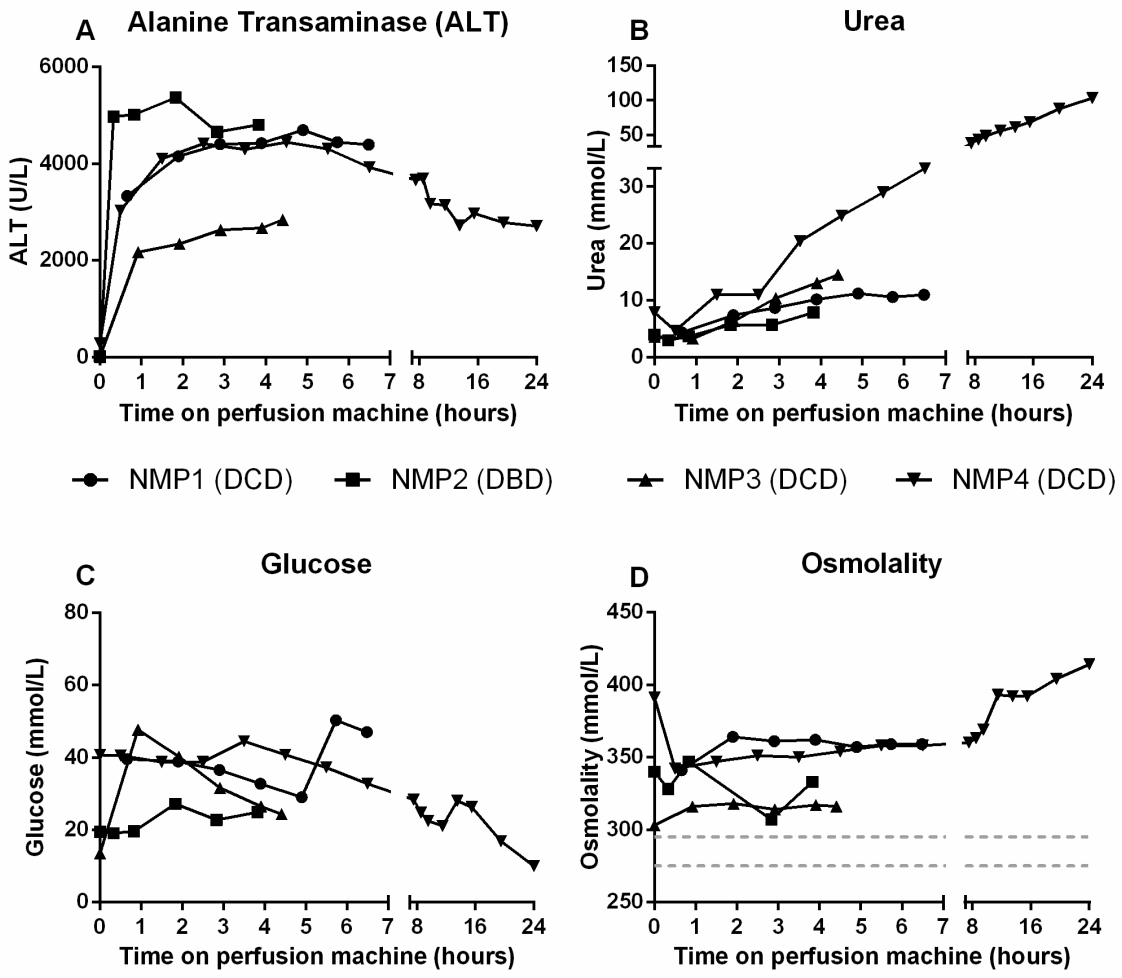

Figure 7.2 Functional parameters liver perfusion up to $\mathbf{2 4}$ hours. A) Alanine Transaminase (U/L), B) Urea $(\mathrm{mmol} / \mathrm{L}), \mathrm{C})$ Glucose $(\mathrm{mmol} / \mathrm{L})$ and D) Osmolality $(\mathrm{mmol} / \mathrm{L})$. The dotted lines reflect the normal range of serum osmolality $275-295 \mathrm{mmol} / \mathrm{L}$.

\section{Discussion}

The results described represent an early experience in NMP of the human liver but already an important lesson has emerged. The functions of the liver are many and complex and NMP is a sophisticated in vitro organ maintenance system. There may be many facets of physiology to consider in determining the effectiveness of in vitro organ support. We found that it is straightforward to establish perfusion with a single pump system (using a portal venous reservoir at a specified height to achieve pressure-regulated flow) and that acid-base homeostasis can be restored quickly. This single pump circuit, similar to that developed in Oxford, is simpler and less expensive than a 2 pump setup but still able to provide the physiological dual blood supply at different pressures (although the portal venous perfusion is non-physiological to the 
extent that it is oxygenated). It was straightforward to set up the centrifugal pump to deliver the arterial perfusate at a fixed pressure with the "extra" perfusate going into the portal venous supply reservoir, which functions as a volume buffer. After starting at $1.4 \mathrm{~L} / \mathrm{min}$, no more than 1 or 2 pump flow adjustments were required and the circuit never ran dry. Centrifugal pumps are less injurious to red blood cells than roller pumps which may be relevant for the development of prolonged perfusion protocols. While there was substantial transaminase release into the perfusion circuit initially, thereafter we observed no further rise. This suggests that ongoing hepatocyte damage is limited. Likewise, bile production was observed. However, the steadily rising urea levels in the circuit, which can only result from the continuation of urea cycle activity in the hepatocytes, and can therefore be considered a parameter of a functional NMP system, contribute substantially to a steady rise in osmolality. This was most strikingly seen in NMP4, successfully perfused for 24 hours. Contributing to hyper-osmolality, very high levels of perfusate glucose were observed in all four liver perfusions which were persistent for $6-12$ hours despite the administration of large doses of insulin: this phenomenon has also been observed in pig livers perfused by the Oxford group in their NMP system (Friend, personal communication). Human tissues are sensitive to osmolar stress; high serum sodium levels in organ donors are linked with adverse transplant outcomes and may be a surrogate for osmolar stress. ${ }^{2}$ Although we have not yet directly measured osmolality in the perfusion circuit, $a$ priori it seems unlikely that it could be lower because the calculated osmolality formula, described by Bhagat et al., used by our laboratory accounts for urea and glucose concentrations and the principle electrolytes. ${ }^{3}$ Additional to an osmotic effect, it has been shown that a high concentration of urea $(100 \mathrm{mmol} / \mathrm{L})$ negatively affects hepatic metabolism via proteolysis inhibition. ${ }^{4}$

The mechanisms underlying high levels of both glucose and urea production remain to be fully elucidated. It is possible that the NMP system stimulates high glycogenolytic activity resistant to insulin and urea production may be driven by the infusion of amino acids in the circuit. We and others have made the assumption that liver homeostasis in NMP may be supported by amino acids. ${ }^{1}$ While yet unproven, there are data to show that L-arginine, for example, reduces preservation injury by stimulation of nitric oxide production. ${ }^{5}$ Further work is needed to determine whether the benefit of amino acid infusion outweighs any deleterious effects. If it does, high levels of urea production may be unavoidable and would need to be addressed in another way. One strategy would be to incorporate a dialyser in the circuit - this could clear both urea and glucose. This would be straightforward in our static setup, and indeed we intend to explore this next, but it would not be feasible in a portable system. If dialysis does prove to be a requisite component of perfusion systems for organ storage beyond a few hours then portable devices in development will need the provision of a connection to dialysis before and/or after transport. 
Finally, one of the main problems in expanding the use of DCD livers for transplantation has been the intractable problem of ischemic cholangiopathy. It would be plausible to consider that osmotic stress in biliary epithelium could be another injury to the biliary tract that might result from long periods of NMP for organ preservation and assessment.

We believe that it would be sensible to maintain physiological osmolality in NMP systems. This could be achieved with the provision of dialysis of the perfusate during NMP, especially for the successful development of perfusion systems beyond 6 hours. 


\section{References}

1. Op den Dries S, Karimian N, Sutton ME, Westerkamp AC, Nijsten MW, Gouw AS, et al. Ex vivo Normothermic Machine Perfusion and Viability Testing of Discarded Human Donor Livers. Am J Transplant. 2013;13(5):1327-35.

2. Totsuka E, Dodson F, Urakami A, Moras N, Ishii T, Lee MC, et al. Influence of high donor serum sodium levels on early postoperative graft function in human liver transplantation: effect of correction of donor hypernatremia. Liver Transplant. 1999;5(5):421-8.

3. Bhagat $\mathrm{Cl}$, Garcia-Webb P, Fletcher E, Beilby JP. Calculated vs measured plasma osmolalities revisited. Clin Chem. 1984;30(10):1703-5.

4. Reinehr R, Gohlke H, Sommerfeld A, Vom Dahl S, Haussinger D. Activation of integrins by urea in perfused rat liver. J Biol Chem. 2010;285(38):29348-56.

5. Yagnik GP, Takahashi Y, Tsoulfas G, Reid K, Murase N, Geller DA. Blockade of the L-arginine/NO synthase pathway worsens hepatic apoptosis and liver transplant preservation injury. Hepatology. 2002;36(3):573-81. 


\section{Chapter 8}

Does normothermic machine perfusion preserve bile ducts as well as it preserves hepatocytes? A study of human donor livers

J Reiling

AH Simpson

CM Campbell

DSR Lockwood

KR Bridle

N Santrampurwala

L Britton

DHG Crawford

CHC Dejong

J Fawcett

Submitted 


\section{Abstract}

\section{Background}

Machine perfusion is an emerging technique in the field of liver transplantation and could potentially aid in the use of marginal donor livers currently deemed unsuitable for transplantation. In the current study we explored the use of normothermic machine perfusion (NMP) and aimed to determine its effect on biliary injury.

\section{Method}

Human donor livers deemed unsuitable for transplantation were perfused under normothermic conditions using a custom made setup. Throughout the perfusions, serial perfusate samples were collected for biochemical and haematological analysis. Furthermore, bile, liver and bile duct tissue was collected during organ retrieval, at the start and end of the perfusion period for assessment of hepatocellular and biliary injury.

\section{Key findings}

Based on lactate clearance during the first two hours of perfusion, two distinct groups of livers could be defined. In addition, lactate clearers $(N=7)$ had significantly lower levels of potassium, aspartate transaminase and $\mathrm{\gamma}$-glutamyl transferase in their perfusate compared to non-lactate clearers $(N=3)$. Following machine perfusion, severe injury of the common bile duct $(C B D)$ was observed in all donor livers. However, when comparing the degree of biliary injury at the level of the CBD to that of the left/right main ducts or the segmental ducts, no correlation was found.

\section{Conclusions}

Lactate clearance correlates well with other parameters of hepatocellular function. NMP was not able to prevent severe biliary epithelial disruption of the CBD. However, there was no correlation between the degree of injury in the CBD and the large intrahepatic bile ducts and therefore a more reliable biomarker of biliary injury is needed to assess the future risk of ischemic type cholangiopathy in the recipients of DCD liver transplants. 


\section{Introductions}

Due to a persistent mismatch between supply of and demand for organ donors, livers with marginal characteristics, such as those from older donors or with hepatic steatosis, are being considered for transplantation. ${ }^{1}$ In addition, donation after circulatory death (DCD) provides an alternative donation pathway for patients not meeting criteria for brain death. The quality of these marginal donor organs is compromised at the start of the donation process and they poorly tolerate an additional period of conventional cold storage. ${ }^{2}$ Criteria for the use of these organs are largely empirical and are imperfect predictors of complications, particularly biliary problems, which are a major limiting factor in the outcome of DCD liver transplantation. ${ }^{3}$ Alternative preservation methods that are better able to maintain, or even improve, organ function and allow for the assessment of organ quality could make more use of these marginal grafts that may otherwise be unsuitable for transplantation.

One such alternative preservation method is the application of ex vivo machine perfusion. Once liver perfusion is established, it is provided with metabolic substrates to support hepatic metabolism and the accumulation of waste products is avoided. Guarrera et al. were the first to successfully implement a hypothermic machine perfusion (HMP) in clinical transplantation. ${ }^{4}$ HMP of donor organs reduced the severity of ischemia-reperfusion injury and facilitated the use of livers (donated after brain death (DBD)) who were already rejected by every other center in their procurement region. ${ }^{5,6}$ Dutkowski et al. furthermore successfully transplanted up to 30 DCD livers with extended warm ischemic times following oxygenated HMP. ${ }^{7,8}$ The most notable finding of this study was that none of the recipients developed ischemic type biliary strictures (ITBS) - despite the prolonged "standoff" times required during procurement by local legislation. ${ }^{8}$ Although results of HMP are encouraging, it does not readily permit assessment of graft viability prior to transplantation.

Machine perfusion under oxygenated normothermic conditions (NMP) offers an opportunity for functional assessment of the liver in a physiological setting; this technique has been shown to be safe and feasible, even allowing perfusion to occur during transport. ${ }^{9,10}$ Currently, lactate clearance, whether or not combined with other factors such as bile production, is most often used to discriminate viable from nonviable grafts. ${ }^{11,12}$ Although initial results are promising, some important questions remain: can bile duct viability be predicted and to what extend does NMP protect the bile ducts from injury? A large animal study previously showed a protective effect of NMP on bile duct physiology, but human data on the effect NMP are currently lacking. In this study, human livers declined for transplantation were perfused under normothermic conditions for an average of six hours. The aim of this study was to assess whether currently used suitability criteria for hepatocellular function could 
discriminate between potential viable and non-viable grafts. In addition, the progression of biliary injury was assessed and the correlation between biliary injury of the common bile duct and large and segmental ducts was studied.

\section{Methods}

\section{Donor livers and organ procurement}

All adult organ donors, in whom the liver was deemed unsuitable for transplantation, were eligible for inclusion in this study. Prior to retrieval, informed consent was obtained from each individual donor family to procure the liver for research purposes. The human ethics committees of the Princess Alexandra Hospital and The University of Queensland approved the study protocol.

Livers were retrieved in a standardized fashion following rapid cooling with University of Wisconsin solution (UW) infused via cannulas inserted in the aorta and portal venous system. In donation after brain death (DBD) donors, 25,000 IU of heparin were administered prior to cross-clamp. In DCD donors, state law in Australia does not permit the administration of heparin prior to the declaration of death. Instead, 50,000 IU of heparin are added to the first liter of cold perfusate. Following the initiation of cold perfusion, the gallbladder was incised in situ and flushed with saline to remove excess bile. In addition, inferior vena cava blood from the donor was collected to serve as perfusate during NMP. Following rapid excision, the liver was placed in a bowl at $4^{\circ} \mathrm{C}$ and both the portal vein and hepatic artery were flushed with a further $1 \mathrm{~L}$ of UW solution. Liver and common bile duct biopsies were collected and the liver was subsequently packed and stored using conventional cold storage during transport.

\section{Normothermic machine perfusion setup}

An oxygenated normothermic perfusion apparatus was assembled locally as previously described. ${ }^{13}$ In brief, cannulas inserted in the portal vein, hepatic artery and inferior vena cava were connected to the circuit and perfusate was re-circulated through this closed system using a single centrifugal pump (ROTAFLOW; Maquet, Rastatt, Germany). The hepatic artery was supplied directly by the pump whereas a reservoir supplied the portal vein generating only a low portal pressure $(<10 \mathrm{mmHg})$. A pediatric oxygenator (Dideco TM Kids D101; Sorin Group, Mirandola, Italy) and a heater cooler oxygenated the perfusate (supplied with 100\% oxygen) and warmed it to $37^{\circ} \mathrm{C}$. A haemofilter (Nephral ST 200, Gambro, Lund, Sweden) was connected to the circuit in order to regulate electrolyte concentrations and hematocrit levels. A scavenger system was used to pump leaked perfusate back into the reservoir. Flow 
probes (Medistim, Oslo, Norway) and pressure-monitoring systems (Edwards Lifesciences, Irvine, CA) were connected to both the portal vein and hepatic artery to allow for real time monitoring.

\section{Organ preparation and priming of circuit}

Prior to connecting the liver to the perfusion machine, a standard back table procedure was performed and side branches of the hepatic artery, portal vein and inferior vena cava were ligated. The inferior end of the inferior vena cava was closed using a continuous suture or stapling device. Cannulae were subsequently inserted in both the hepatic artery (16 F, Medtronic, Minneapolis, MN) and the inferior vena cava (34F Medtronic, Minneapolis, MN). The portal venous cannula (10-12 F, Maquet, Rastatt, Germany), inserted during organ retrieval, was left in situ. The cystic artery was ligated and a cannula was inserted via the cystic duct into the common bile duct to allow for the collection of bile. A clip was placed over the distal end of the common bile duct to prevent leakage. Immediately prior to connection to the perfusion machine, $2 \mathrm{~L}$ of cold saline ( $0.9 \%$ sodium chloride) was flushed through the liver to remove residual UW solution.

The circuit was primed using Albumex ${ }^{\circledR} 4$ (Australian Red Cross Blood Service) and/or Plasma-lyte 148 (Baxter, Old Toongabbie, Australia). Autologous blood was subsequently added and the circuit was run for 30-60 minutes to correct acid base balance and achieve a hematocrit $>20 \%$ by using the haemofilter. Furthermore, calcium gluconate was added to the perfusate if a citrate-based anticoagulation solution was used to preserve donor blood during transport. During perfusion, insulin (100 IU/hour), amino acids (20 ml/hour), taurocholic acid (1\%, $7 \mathrm{ml} /$ hour) and prostacyclin ( $8 \mu \mathrm{g} /$ hour) were continuously infused. Heparin was administered based on the activated clotting time.

Throughout perfusion, hepatic artery pressure was maintained below $80 \mathrm{mmHg}$ and portal pressure less than $10 \mathrm{mmHg}$. This generated a flow rate between $300-370 \mathrm{ml} / \mathrm{min}$ in the hepatic artery and $550-1200 \mathrm{ml} / \mathrm{min}$ in the portal vein.

\section{Assessment of liver function}

During the perfusions, serial perfusate samples were collected from the arterial sampling port. During the first hour, blood gas analysis was performed at 10-minute intervals and at 30-minute intervals thereafter. More extensive biochemical and hematological analysis was performed on samples collected every hour. Each parameter is expressed as the level of increase or decrease from baseline to correct for differences resulting from the use of donor blood. A liver biopsy was performed prior to connecting the liver to the circuit as well as at the end of perfusion to perform 
histological analysis. Bile was collected at hourly intervals throughout perfusion. Lactate clearance has previously been used to determine suitability for transplantation following NMP. ${ }^{11}$ Based on these criteria, livers included in this study were assigned to the lactate clearance group if lactate levels were below $2 \mathrm{mmol} / \mathrm{L}$ within 2 hours and to the non-lactate clearance group if they failed do so within that time frame.

\section{Assessment of bile duct injury}

Lactate dehydrogenase (LDH) in bile was measured as a biomarker of biliary injury using a commercially available kit (Bio scientific Corporation, Austin, TX, USA). ${ }^{14}$ In addition, biopsies of the common bile duct were collected at the start and end of perfusion. At the conclusion of the perfusion period, the liver was fixed in $10 \%$ buffered formalin. Serial biopsies were subsequently taken from the biliary tree at the level of the common bile duct (CBD), left/right main duct and segmental ducts. The distal $3 \mathrm{~mm}$ of the CBD was discarded because the vascular clip, applied to prevent leakage during machine perfusion, had damaged this section of the duct. The bile duct biopsies were embedded in paraffin and stained with hematoxylin and eosin. An expert pathologist performed a blinded semi-quantitative histological scoring as previously described by Hansen et al. and op den Dries et al.. ${ }^{15,16}$ The following components were assessed: biliary epithelium, mural stroma, the peribiliary vascular plexus and the peri-luminal and deep peribiliary glands. Each component, apart from mural stromal necrosis, was scored as: 0 ; no sign of injury, 1; less than $50 \%$ affected, 2: $>50 \%$ affected. Mural stroma necrosis was graded 0 : no injury, $1:<25 \%$ of ductular wall affected, 2: $25-50 \%$ of ductular wall affected, 3: $50-75 \%$ of ductular wall affected and $4:>75 \%$ of ductular wall affected.

\section{Measurement of blood-biliary-barrier permeability}

The blood-biliary-barrier (BBB) is responsible for the separation of bile from the blood stream and is formed by neighbouring cholangiocytes joined together by tight junctions. We assessed the integrity of this barrier during two liver perfusions using Horseradish Peroxidase (HRP). ${ }^{17}$ In short, 4500U HRP (HRP, P8250, Sigma-Aldrich, St. Louis, MO, USA) dissolved in $900 \mu \mathrm{L}$ of sterile water was administered to the circuit and bile was collected $1,5,10,15,20$ and 30 minutes following administration. A commercially available assay kit was later used to determine HRP activity in bile (Amplex red Assay kit, \#A22188, Thermo Fisher Scientific, Waltham, MA, USA). Ultimately, the concentration of HRP in bile was multiplied by the volume of bile produced at each time point to generate the total amount of HRP units excreted. 


\section{Statistical analysis}

Continuous variables are expressed as median (interquartile range) and categorical variables as frequency (\%). A Mann-Whitney test, Pearson chi-square test or Fisher's exact test were performed where appropriate to compare the lactate clearance to non-lactate clearance groups. A Wilcoxon matched-pair test was performed when repeated samples from the same liver were compared. A P-value $<0.05$ was considered significant. SPSS Statistics for Macintosh, Version 23.0 (IBM Corp. IMB SPSS statistics, Armonk, NY) was used to perform the statistical analysis.

\section{Results}

\section{Organ donors}

Between March 2014 and May 2016, ten livers that were declined for transplantation were procured and perfused under normothermic conditions (Table 8.1). In addition, five livers (3 DBD, 2 DCD) were retrieved but not perfused due to unavailability of staff. These livers were retrieved and either directly placed in formalin or stored using conventional cold storage and transferred into formalin nine hours later. Donor characteristics of the livers that were not perfused can be found in Suppl. Table S8.1.

Table 8.1 Characteristics of donors used for NMP.

\begin{tabular}{|c|c|c|c|c|}
\hline & $\begin{array}{l}\text { Total }(n=10) \\
\operatorname{nr}(\%), \\
\text { median (IQR) }\end{array}$ & $\begin{array}{l}\text { Lactate clearers } \# \\
\qquad(\mathrm{n}=7) \\
\mathrm{nr}(\%), \\
\text { median (IQR) }\end{array}$ & $\begin{array}{l}\text { Non-lactate clearers } \\
(\mathrm{n}=3) \\
\mathrm{nr}(\%), \\
\text { median (IQR) }\end{array}$ & $p$-value \\
\hline Age (Years) & $60(50-62)$ & $60(56-63)$ & $50(49-60)$ & 0.3 \\
\hline Gender (Male) & $7(70 \%)$ & $5(71 \%)$ & $2(67 \%)$ & $>0.99$ \\
\hline $\mathrm{BMI}\left(\mathrm{Kg} / \mathrm{m}^{2}\right)$ & $25(21-28)$ & $25(22-26)$ & $28(21-37)$ & 0.5 \\
\hline Type (DCD) & $8(80 \%)$ & $7(100 \%)$ & $1(33.3 \%)$ & 0.07 \\
\hline Cause of death & & & & 0.3 \\
\hline Stroke & $6(60 \%)$ & $3(42.9 \%)$ & $3(100 \%)$ & \\
\hline Hypoxic brain injury & $2(20 \%)$ & $2(28.6 \%)$ & $0(0 \%)$ & \\
\hline Other & $2(20 \%)$ & $2(28.6 \%)$ & $0(0 \%)$ & \\
\hline Reason declined & & & & 0.2 \\
\hline DCD + age & $7(70 \%)$ & $6(85.7 \%)$ & $1(33.3 \%)$ & \\
\hline DCD and logistics & $1(10 \%)$ & $1(14.3 \%)$ & $0(0 \%)$ & \\
\hline Steatosis/NASH & $2(20 \%)$ & $0(0 \%)$ & $2(67 \%)$ & \\
\hline $\operatorname{ALT}(\mathrm{U} / \mathrm{L})$ & $57(35-132)$ & $64(14-211)$ & $49(45-100)$ & 0.95 \\
\hline WIT (min) & $19(16-25)$ & $20(17-26)$ & $15^{*}$ & 0.5 \\
\hline $\mathrm{CIT}$ (min) & $330(220-558)$ & $260(194-465)$ & $550(309-745)$ & 0.2 \\
\hline Perfusion (min) & $360(314-390)$ & $360(360-480)$ & $330(240-360)$ & 0.1 \\
\hline
\end{tabular}

\# Lactate clearers: lactate concentration below $2 \mathrm{mmol} / \mathrm{L}$ within 2 hours of perfusion * only one DCD liver included in this group. Abbreviations: BMI: body mass index, DCD: donation after circulatory death, NASH: non-alcohol steatohepatitis, ALT: alanine transaminase, WIT: warm ischaemic time, CIT: cold ischaemic time. 
Of the livers that were perfused, eight livers were procured from DCD donors and two from DBD donors with a median donor age of 60 (50-62) years. The main reasons the livers were not used for transplantation was the presence of severe steatosis in the DBD donor livers, and advanced age of DCD donors. Overall, stroke was the most common cause of death and the majority of donors were male. When we stratified the livers by their ability to clear lactate, three were identified as likely non-lactate clearers. Both DBD livers included in this study failed to clear lactate, which persisted throughout the perfusion period. Warm ischemic time (WIT), defined as the period between the onset of significant ischemia (oxygen saturation $<50 \%$ or systolic blood pressure below $50 \mathrm{mmHg}$ ) and the start of cold perfusion, was 19 (16-25) minutes. The livers were stored for 5.5 hours on average with a perfusion period of up to 24 hours.

\section{Perfusate parameters distinguishing lactate clearers and non-lactate clearers}

Throughout the machine perfusion period, serial blood samples were collected. Lactate clearance defined two distinct groups of donor livers (Figure 8.1). In addition to lactate production, non-lactate clearers $(n=3)$ released significantly more potassium, $\gamma$-glutamyl transferase (GGT) and aspartate transaminase (AST) in the perfusate than livers in the lactate clearers group $(n=7)$ following two hours of perfusion (Table 8.2). Furthermore, non-lactate clearers produced less urea compared to lactate clearers $(2.1(1.7-2.4) \mathrm{U} / \mathrm{L}$ versus $3.3(2.8-6.8) \mathrm{U} / \mathrm{L}(p=0.02))$. Following 4 hours of perfusion, GGT levels persisted to be elevated with non-lactate clearers releasing 119 (13-173) U/L compared to 35 (27-55) U/L in the lactate clearance group $(p=0.01)$. Only 8 livers were perfused for at least six hours. At the end of the perfusion period, lactate, potassium AST, GGT and alkaline phosphatase tended to be increased in the perfusate of non-lactate clearers ( $p=0.07$ for each).

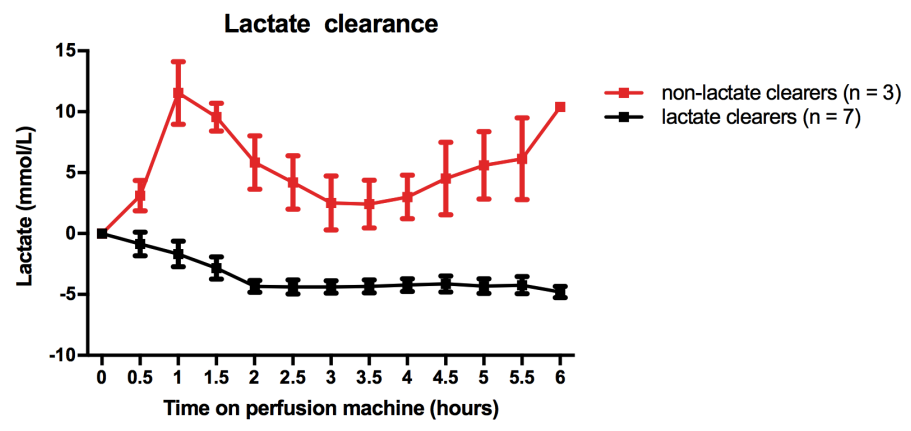

Figure 8.1 Lactate clearance during normothermic machine perfusion. During normothermic machine perfusion, the concentration of lactate $(\mathrm{mmol} / \mathrm{L})$ in perfusate was measured in thirty-minute intervals. Data are depicted as median (inter quartile range) increase or decrease from baseline with lactate clearers $(n=7)$ shown in black and non-lactate clearers $(n=3)$ shown in red. 


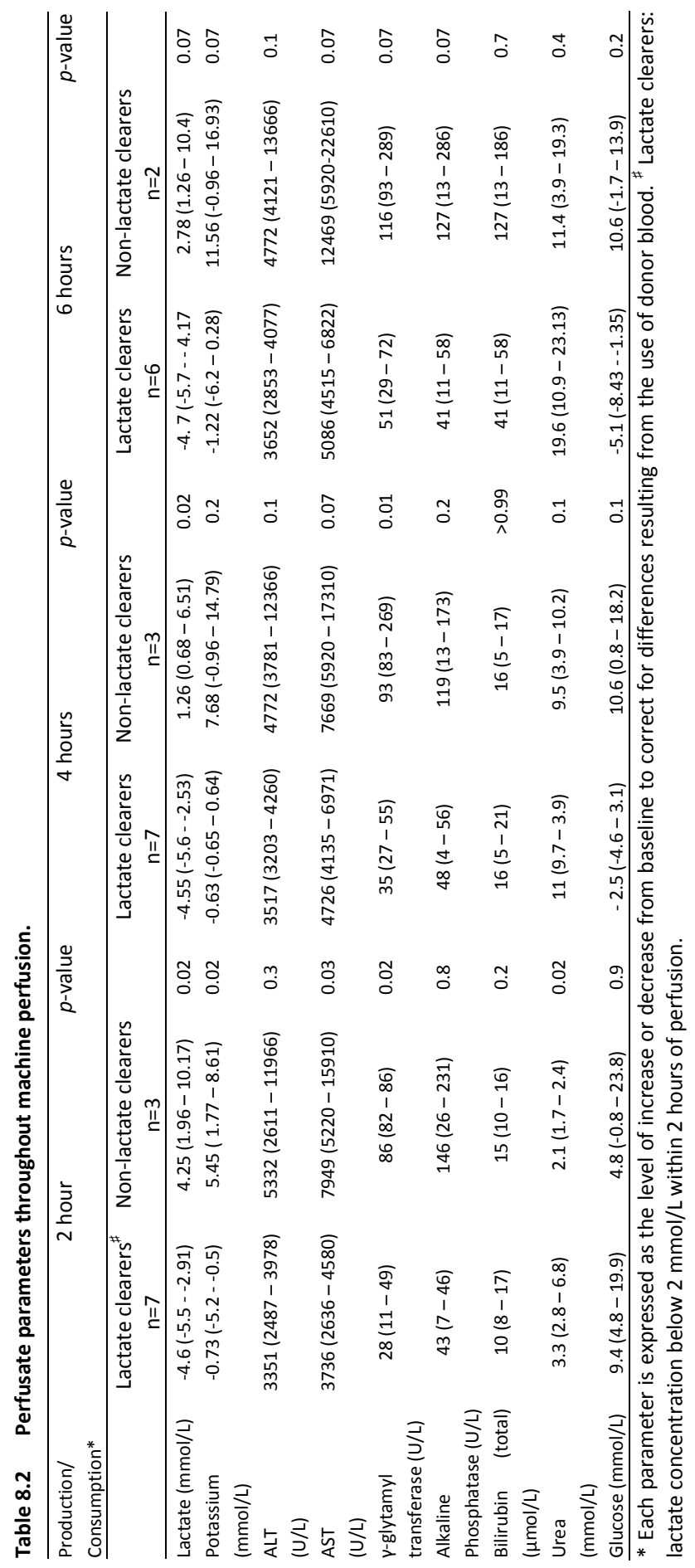


Histological assessment of the liver at the end of the perfusion period showed that hepatocellular morphology was preserved in all perfused livers without evidence of necrosis (Figure 8.2). The two DBD livers in the non-lactate clearance group showed over $90 \%$ macrovesicular steatosis at the end of NMP. The third non-viable graft, from a DCD donors, showed severe portal proliferation and inflammatory infiltrates on biopsy taken at retrieval (data not shown).

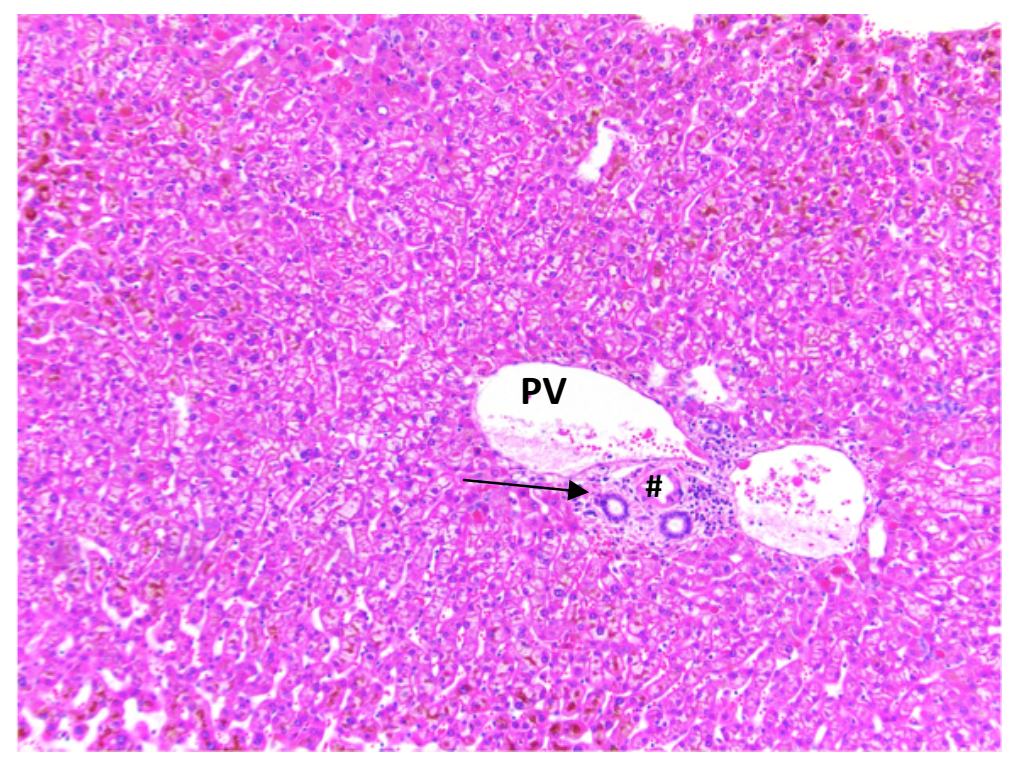

Figure 8.2 Histology of liver parenchyma following normothermic machine perfusion. Liver parenchymal biopsy stained with haematoxylin and eosin of DCD liver graft that cleared lactate at the end of normothermic machine perfusion \#: hepatic artery, PV: portal vein.

\section{Glucose production of DCD donors livers compared to DBD donors livers during NMP}

During machine perfusion, DCD livers in the lactate clearance group showed a distinct pattern of glucose release and consumption. Within the first hour of perfusion, glucose concentrations rose by $15.1 \mathrm{mmol} / \mathrm{L}$ despite the administration of insulin (Figure 8.3). In addition, glucose concentrations in venous blood were increased compared to those in blood collected from the arterial sampling port during this period. Throughout the remaining five hours of perfusion, glucose was gradually consumed by these DCD livers to levels below those at the start of the perfusion. DBD livers in the non-lactate clearance group on the other hand did not respond to the administration of insulin and glucose levels remained largely unchanged throughout the perfusion. 


\section{Glucose production/consumption}

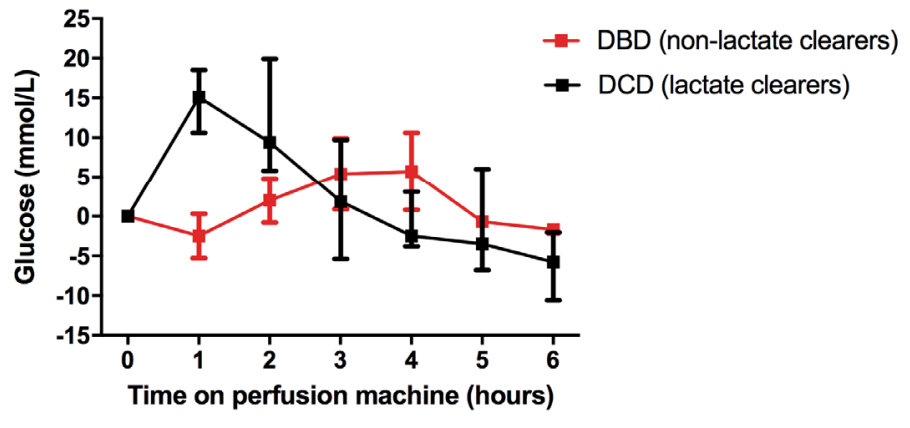

Figure 8.3 Glucose production and consumption of DCD donor livers compared to DBD donor livers during normothermic machine perfusion. During normothermic machine perfusion, the concentration of glucose $(\mathrm{mmol} / \mathrm{L})$ in perfusate was measured every hour. Data are depicted as median (inter quartile range) increase or decrease from baseline with DCD livers (lactate clearers) shown in black and DBD liver grafts (non-lactate clearers) shown in red. DCD: donation after circulatory death; DBD: donation after brain death.

\section{Progressive biliary injury from organ retrieval until end of machine perfusion period}

Each liver produced bile with volumes varying from less than $1 \mathrm{ml}$ per hour to more than $10 \mathrm{ml}$ per hour. The levels of LDH in bile at the start of perfusion as a biomarker of biliary injury were significantly increased compared to samples collected at the time of organ retrieval (Figure 8.4). However towards the end of the machine perfusion period, LDH concentrations decreased to a median value of 3,555 (573-6,674) U/L ( $p=0.02)$.

CBD specimens collected at the time of organ donation $(n=9)$, at the end of cold perfusion $(n=5)$ and at the end of the perfusion period $(n=10)$ were systematically assessed for signs of injury (Table 8.3). Overall, no thrombi were observed in the peribiliary plexus and there were no signs of inflammation at either of the three time points. At the time of organ retrieval, severe injury to the biliary epithelium was already evident in the majority of CBD biopsies. At the end of machine perfusion, each CBD sample displayed over $50 \%$ loss of the epithelial cell lining. At the time of organ retrieval, the majority of CBD samples showed no signs of necrosis of the mural stroma. However, at the end of NMP, 8 (80\%) CBD samples showed signs of severe mural necrosis with more than $75 \%$ of the duct wall affected. Similarly, in $5(50 \%)$ of the CBD sections arteriolonecrosis of the vascular plexus was observed at the end of NMP although no damage was observed at the time of organ donation $(p=0.006)$. Bleeding of the mural stroma affecting over $50 \%$ of the duct wall was observed in two- 
thirds of CBD sections at the time of donation. At the end of the machine perfusion period, 9 (90\%) of the CBD samples showed severe (>50\% of the duct wall) mural bleeding. The deep peribiliary glands remained largely preserved during perfusion, with only $2(20 \%)$ of the CBD sections displaying $>50 \%$ of cell loss.

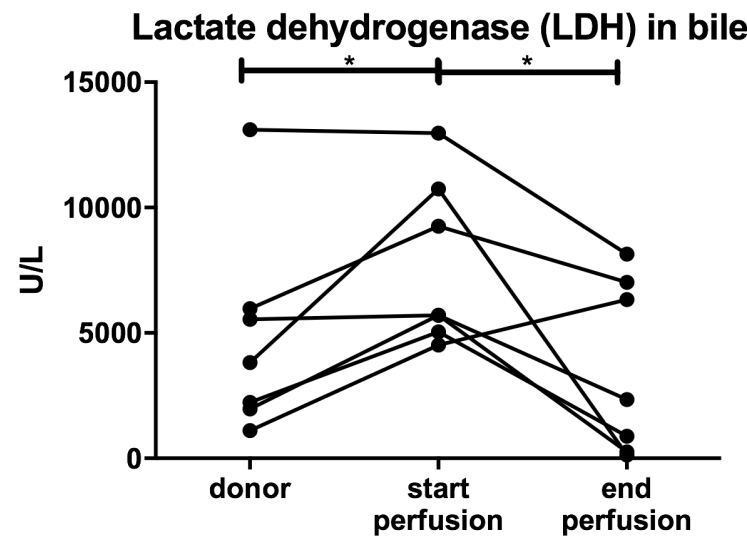

Figure 8.4 Levels of lactate dehydrogenase in bile. Lactate dehydrogenase (LDH, U/L) was measured in bile at the time of organ donation, at the start and end of machine perfusion, as a biomarker of biliary injury. The concentration of LDH is shown from every individual liver at each time point. A Wilcoxon paired comparison test was performed to compare the concentrations at different time points and a $p$-value of $<0.05$ was considered significant. ${ }^{*} p<0.05$.

Poor correlation between injury to the common bile duct and intrahepatic bile ducts

Livers collected directly after organ retrieval (no cold storage, $n=3$ ), following an ischemic period of 9 hours $(n=2)$ or at the end of the perfusion period $(n=10)$ were placed in formalin and biopsies at different levels of the biliary tree were collected. A semi-quantitative assessment of bile duct injury was performed and overall, there were no signs of inflammation of the bile duct wall and thrombi could not be detected (Figure 8.5). The majority of CBD sections displayed severe loss of biliary epithelial cells (epithelial lining absent from $>50 \%$ of duct). In contrast, the majority of livers had segmental ducts with no sign of epithelial injury (0/15 CBD versus 11/14 segmental ducts, $p=0.001$ ). Similarly, the degree of bleeding and necrosis of the mural stroma was significantly less severe amongst the intrahepatic bile ducts. Mural stromal necrosis (>25\% of the duct affected) was present in 12 (80\%) CBD sections compared to $1(7 \%)$ of the segmental biopsies $(p=0.004)$. Mural bleeding affecting more than $50 \%$ of the duct was found in $6(40 \%)$ CBD sections compared to $2(13 \%)$ of the main ducts and none of the segmental duct sections. The peribiliary plexus remained largely 
intact overall, but the degree of injury to the CBD was found to be more severe than that of the segmental ducts $(p=0.052)$. Consistent with our findings of mural stromal damage, periluminal peribiliary glands were more severely affected in the common bile duct sections. The degree of injury to the deep peribiliary glands was not found to be different between the bile duct biopsies.

Table 8.3 Common bile duct injury throughout the perfusion process.

\begin{tabular}{|c|c|c|c|c|}
\hline & $\begin{array}{l}\text { Donor } \\
(\mathrm{N}=9)\end{array}$ & $\begin{array}{l}\text { End of cold storage } \\
\qquad(\mathrm{N}=5)\end{array}$ & $\begin{array}{l}\text { End of perfusion } \\
\qquad(\mathrm{N}=10)\end{array}$ & $p$-value \\
\hline Biliary epithelium & & & & 0.1 \\
\hline Grade 0 & $2(22 \%)$ & $0(0 \%)$ & $0(0 \%)$ & \\
\hline Grade 1 & $1(11 \%)$ & $0(0 \%)$ & $0(0 \%)$ & \\
\hline Grade 2 & $6(67 \%)$ & $5(100 \%)$ & $10(100 \%)$ & \\
\hline Necrosis of mural stroma & & & & 0.007 \\
\hline Grade 0 & $5(57 \%)$ & $2(40 \%)$ & $0(0 \%)$ & \\
\hline Grade 1 & $2(22 \%)$ & $0(0 \%)$ & $0(0 \%)$ & \\
\hline Grade 2 & $1(11 \%)$ & $2(40 \%)$ & $0(0 \%)$ & \\
\hline Grade 3 & $1(11 \%)$ & $1(20 \%)$ & $2(20 \%)$ & \\
\hline Grade 4 & $0(0 \%)$ & $0(0 \%)$ & $8(80 \%)$ & \\
\hline Intramural bleeding & & & & 0.03 \\
\hline Grade 0 & $2(22 \%)$ & $0(0 \%)$ & $0(0 \%)$ & \\
\hline Grade 1 & $1(11 \%)$ & $0(0 \%)$ & $1(10 \%)$ & \\
\hline Grade 2 & $6(67 \%)$ & $5(100 \%)$ & $9(90 \%)$ & \\
\hline Vascular lesions & & & & 0.006 \\
\hline Grade 0 & $9(100 \%)$ & $4(80 \%)$ & $4(40 \%)$ & \\
\hline Grade 1 & $0(0 \%)$ & $0(0 \%)$ & $1(10 \%)$ & \\
\hline Grade 2 & $0(0 \%)$ & $0(0 \%)$ & $0(0 \%)$ & \\
\hline Grade 3 & $0(0 \%)$ & $1(20 \%)$ & $5(50 \%)$ & \\
\hline Peri-luminal peribiliary glands & & & & 0.7 \\
\hline Grade 0 & $2(22 \%)$ & $1(20 \%)$ & $1(10 \%)$ & \\
\hline Grade 1 & $1(11 \%)$ & $0(0 \%)$ & $2(20 \%)$ & \\
\hline Grade 2 & $6(67 \%)$ & $4(80 \%)$ & $7(70 \%)$ & \\
\hline Deep peribiliary glands & & & & $>0.99$ \\
\hline Grade 0 & $3(38 \%)$ & $1(25 \%)$ & $5(50 \%)$ & \\
\hline Grade 1 & $4(50 \%)$ & $1(25 \%)$ & $3(30 \%)$ & \\
\hline Grade 2 & $1(13 \%)$ & $2(50 \%)$ & $2(20 \%)$ & \\
\hline
\end{tabular}

\section{Permeability of the blood biliary barrier during machine perfusion}

In this study, the integrity of the BBB was assessed to investigate if it would be involved in the development of biliary injury during NMP (Figure 8.6). HRP was administered once during the perfusion of the seventh liver (NMP7) and twice during perfusion of the tenth liver (NMP10). The biliary activity of HRP could be measured in each sample, with the highest output encountered three and a half hours into the perfusion of NMP10. During NMP7, the output of HRP increased during the first ten minutes after which the output plateaued. During NMP 10, the output of HRP 10-minutes following administration was 3.4 fold lower after 5.5 hours of NMP compared to 3.5 hours of perfusion. 

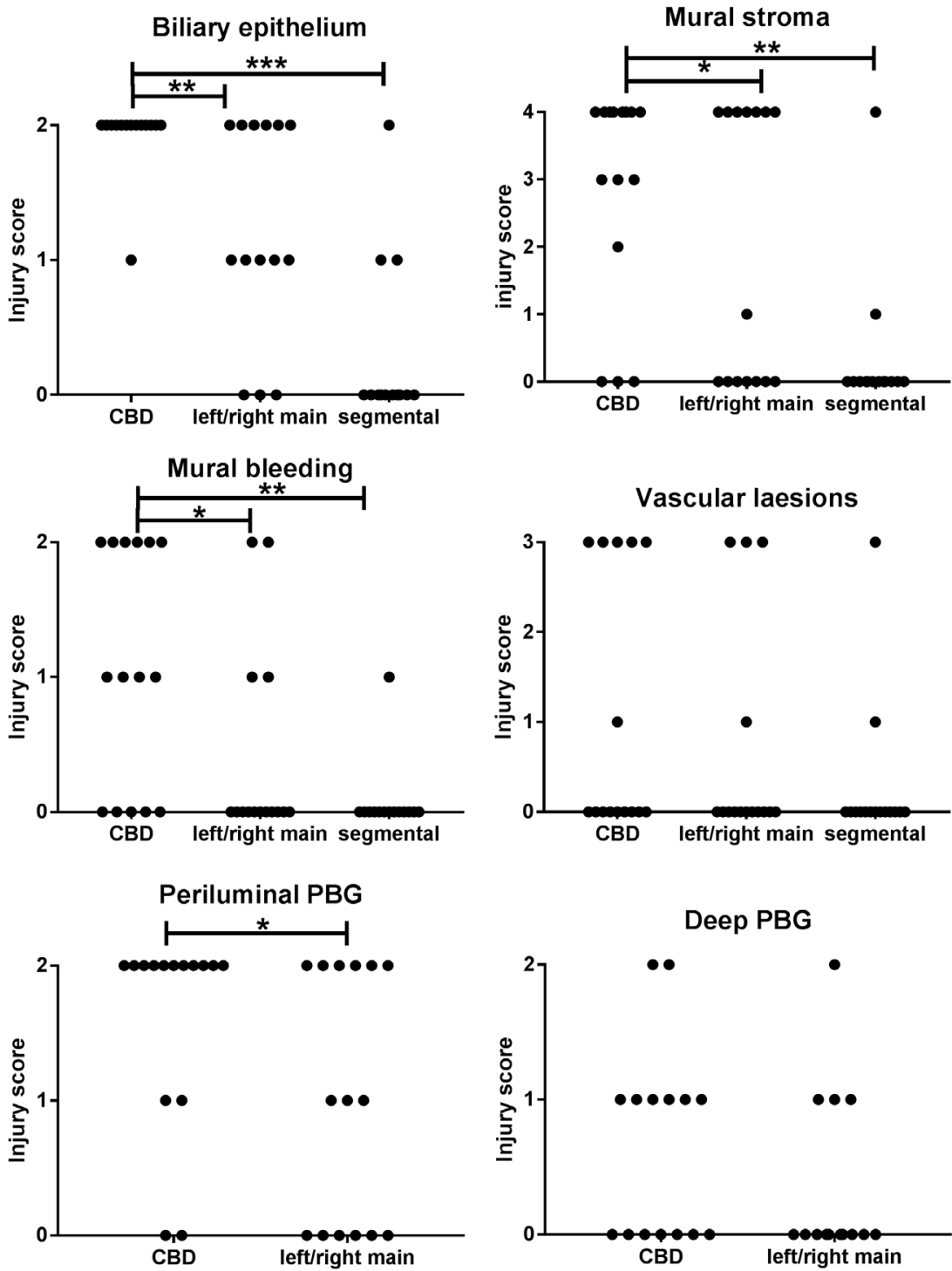

Figure 8.5 Histological scoring of bile duct injury at the level of the common bile duct and the large intrahepatic ducts. Liver biopsies were collected from the common bile duct (CBD), left/right main duct and the segmental ducts of each liver $(n=15)$ and a semi-quantitative biliary injury was performed. A Wilcoxon paired comparison test was performed to compare the concentrations at different time points and a $p$-value of $<0.05$ was considered significant. ${ }^{*} p<0.05,{ }^{* *} p<0.01$. 


\section{Output of Horseradish Peroxidase during NMP}

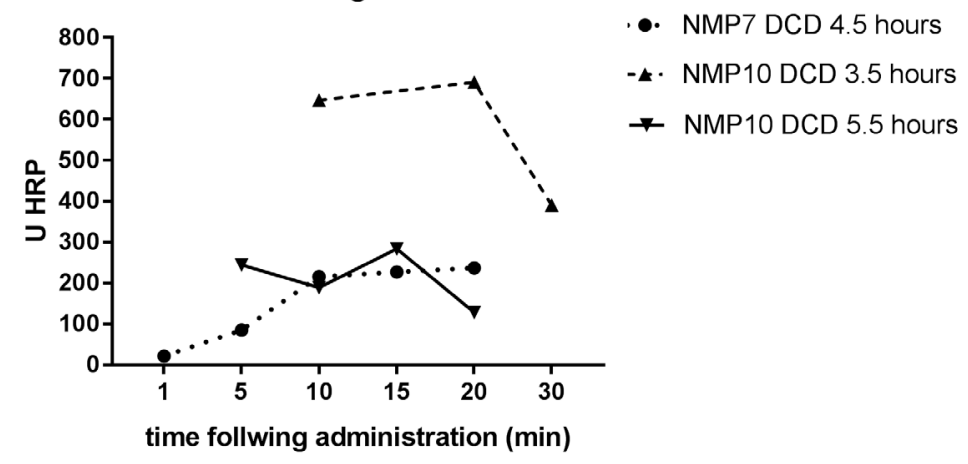

Figure 8.6 Assessment of blood-biliary-barrier integrity during normothermic machine perfusion. Horseradish peroxidase (HRP, 4500U) was administered to the perfusion circuit during the perfusion of NMP7 and NMP10 to assess the integrity of the blood-biliary-barrier. Bile was subsequently collected and the concentration of HRP in bile was assessed. Ultimately, the output of HRP was determined by multiplying the concentration of HRP by the volume of bile produced.

\section{Discussion}

Livers donated after circulatory death are often unused as they have been associated with inferior graft outcomes and the development of biliary complications. ${ }^{18,19}$ The application of machine perfusion has been proposed as a way to better preserve organ function of marginal donor livers such as those donated after circulatory death. In the current study, livers deemed unsuitable for transplantation were perfused under normothermic conditions for an average of six hours. Based on lactate clearance within two hours of perfusion seven out of ten livers could have potentially been used for transplantation. Lactate clearance is likely to reflect hepatocellular viability given that this is the most abundant cellular population in the liver.

In the current study, lactate clearance to below $2 \mathrm{mmol} / \mathrm{L}$ within 2 hours correlated well with other indicators of liver function such as AST, potassium release and GGT levels at the same time point. Liver morphology was preserved during NMP. Currently several centres have perfused human livers under normothermic conditions but criteria used to determine viability are variable. Sutton et al. proposed to use the volume of bile produced during perfusion ( $>20 \mathrm{~g}$ over 6 hours) as this process requires both functional hepatocytes and cholangiocytes. ${ }^{20}$ Perera et al. and Watson et al. described the first two successful liver transplants of NMP perfused DCD grafts 
previously deemed unsuitable for transplantation. ${ }^{11,12}$ Both groups used lactate clearance to determine if the graft could be safely used for transplantation. In a later series by the Birmingham group, livers that failed to clear lactate but produced bile were also considered for transplantation. ${ }^{21}$ In addition, they had to fulfil two out the three following requirements: perfusate $\mathrm{pH}>7.3$, satisfactory macroscopic appearance or arterial flow $>150 \mathrm{ml} / \mathrm{min}$ and portal flow $>500 \mathrm{ml} / \mathrm{min}$. Of the six livers that were previously declined by other centres, five met viability criteria and were safely transplanted without developing early allograft dysfunction. ${ }^{21}$

All livers included in the present cohort produced bile, even when lactate clearance was impaired, and bile production may therefore not be a reliable indicator of graft viability. Conversely, in a recent study by Mergental et al, only one liver produced more than $20 \mathrm{~g}$ of bile during NMP but all five grafts had excellent graft function. ${ }^{21}$

Throughout the perfusion period we observed a distinct pattern of glucose production and consumption in the livers that were deemed viable. This phenomenon of glucose release by DCD livers within the first hour of perfusion has been observed by other groups $^{12,22}$ and is likely a result of glycogenolysis of ischaemic hypothermic liver grafts. ${ }^{23}$ Consistent with this was the observation that glucose concentrations in hepatic venous blood samples were increased compared to perfusate collected from the arterial sampling port during machine perfusion. During NMP, glucose levels decreased and this was likely from the restoration of hepatocyte glycogen stores, as was also observed by Mergental et al.. ${ }^{21}$ Interestingly, the two DBD livers perfused in the current study were both considered non-viable and this pattern of glucose clearance was not observed.

Although we found that lactate clearance correlated well with other parameters of hepatocellular function, it might not correlate with viability of the bile duct in DCD grafts. After machine perfusion, regardless of the metabolic performance of the liver, the majority of $C B D$ sections showed evidence of mural stromal necrosis, mural bleeding and loss of peri-biliary glands. However the deep peribiliary glands remained largely preserved which could potentially allow for regeneration. Previous work by op den Dries et al. found a strong correlation between the degree of bile duct necrosis, biliary plexus and peribiliary gland injury at the end of cold storage and the development of ITBS. ${ }^{16}$ It is currently unknown to what extent the degree CBD damage at the end of machine perfusion correlates with the development of ITBS. It is however likely that the same components still play a role as biliary injury is aggravated upon reperfusion. ${ }^{24}$ The extended period of cold storage prior to the establishment of NMP might have played a role in the degree of biliary injury observed in this study. The results of the COPE trial, where livers were perfused starting very soon after procurement, will shed further light on this important question.

As the perfused livers were not transplanted following NMP, it provided us with a unique opportunity to assess the degree of damage throughout the biliary tree. Compared to biopsies collected from the $\mathrm{CBD}$, the main intrahepatic ducts as well as 
segmental ducts showed significantly less injury to all scored components apart from the deep peri-biliary glands. These findings are in stark contrast to those previously reported by Karimian et al.. ${ }^{25}$ One of the main differences to our study cohort is that the livers included in their study were not re-perfused and samples were collected at the end of cold storage. Reperfusion aggravated biliary injury and therefore the overall degree of injury observed in our study is much greater. When assessing only those livers that did not undergo NMP in the present cohort, the degree of injury to the $\mathrm{CBD}$ was lower. Furthermore, no differences were found between the degree of injury to the $C B D$, main ducts or the segmental ducts of these unperfused grafts. The baseline characteristics did not differ between unperfused livers and those that underwent NMP, and availability of staff was the only reason they did not undergo NMP. The discrepancy in injury severity underlines the need for a more reliable biomarker for biliary injury, as assessment of CBD biopsies does not reveal the true extent of the injury to the large bile ducts.

NMP has previously been shown to have a protective effect on bile ducts as it reduced the degree of biliary injury and enhanced regeneration in animal studies. ${ }^{26,27}$ Cholangiocyte cell function improved and biomarkers of biliary injury were significantly lower in the groups of rats undergoing NMP. In addition, porcine livers subjected to ten hours of sanguineous NMP followed by perfusion with whole blood for 24 hours only displayed mild biliary injury with diffuse regeneration of both the epithelial cell lining and the peribiliary glands. No study conducted in humans has thus far been able to determine the effect of NMP on the development of biliary injury and stricture formation. Results from larger studies are needed to determine whether NMP is able to prevent the development of ITBS.

Increased permeability of the blood biliary barrier has previously been associated with biliary stricture formation as it allowed for bile to leak into the portal tract area inducing fibrogenesis. ${ }^{28}$ Furthermore, staining for tight junction proteins Zo-1 and Claudin-1 in CBD sections collected from organ donors throughout the donation process showed severely altered tight junction architecture (24). In the current study, HRP flux into bile was examined to see if it might be useful in determining the integrity of the barrier during two perfusions. We were able to determine the activity of HRP in bile but the interpretation of these results is not straightforward. Firstly, sufficient amounts of bile need to be produced to conduct the assay. Therefore it was not possible to perform the assay during every liver perfusion included in this cohort. Furthermore, as extension tubing or paediatric feeding tubes are used to facilitate bile collection outside the main sterile field, the concentration of bile collected might reflect what has been excreted several minutes earlier. This is of particular importance because it has been reported that HRP enters bile via the para-cellular route, across tight junctions, within 5-7 minutes. ${ }^{29}$ A late peak in biliary HRP, observed 20-25 minutes following administration, is thought to reflect transcytosis rather than 
para-cellular movements. Therefore, different ways to functionally assess the biliary integrity need to be considered in future experiments.

The results of this study are in line with previous studies and highlight the impact NMP can have on the total number of livers available for transplantation. In Australia, organ retrieval and subsequent transplantation are generally performed by the same centre which would allow for the matching of NMP perfused marginal livers to low risk recipients. Over the last 2 years, 270 liver grafts from adult donors were declined for transplantation in Australia and $54 \%$ of those were from DCD donors. If $70 \%$ of DCD grafts could be recovered using NMP, this would increase the donor pool by $16 \%$. This figure is similar to the predictions made in the UK where an estimated $15 \%$ increase could be achieved if NMP were implemented. ${ }^{21}$

The current case series of ten perfused human donor livers is relatively small. However the perfusions were performed over a period of more than two years thereby making it a valuable resource. As only two DBD donor livers were included in the current study it was difficult to compare the different donor types.

Results from this study show that lactate clearance is a suitable determinant of hepatocellular viability. Biliary viability is however important when considering the use of DCD livers and this is currently not assessed prior to transplantation. As biliary injury to the $\mathrm{CBD}$ does not correlate with the degree of injury to the main intrahepatic bile ducts, new biliary biomarkers should be explored in order to determine which grafts are unsuitable for transplantation due to a high risk of ITBS formation 


\section{References}

1. Durand F, Renz JF, Alkofer B, Burra P, Clavien PA, Porte RJ, et al. Report of the Paris consensus meeting on expanded criteria donors in liver transplantation. Liver Transpl. 2008;14(12):1694-707.

2. Monbaliu D, Liu Q, Vekemans K, Roskams T, Pirenne J. Potentiation of adverse effects of cold by warm ischemia in circulatory death donors for porcine liver transplantation. Transplant Proc. 2012;44(9): 2874-9.

3. Laing RW, Scalera I, Isaac J, Mergental H, Mirza DF, Hodson J, et al. Liver transplantation using grafts from donors after circulatory death: A propensity-matched study from a single centre. Am J Transplant. 2016.

4. Guarrera JV, Henry SD, Samstein B, Odeh-Ramadan R, Kinkhabwala M, Goldstein MJ, et al. Hypothermic machine preservation in human liver transplantation: the first clinical series. Am J Transplant. 2010;10(2):372-81.

5. Guarrera JV, Henry SD, Samstein B, Reznik E, Musat C, Lukose TI, et al. Hypothermic machine preservation facilitates successful transplantation of "orphan" extended criteria donor livers. Am J Transplant. 2015;15(1):161-9.

6. Henry SD, Guarrera JV. Protective effects of hypothermic ex vivo perfusion on ischemia/reperfusion injury and transplant outcomes. Transplant Rev. 2012;26(2):163-75.

7. Dutkowski P, Polak WG, Muiesan P, Schlegel A, Verhoeven CJ, Scalera I, et al. First Comparison of Hypothermic Oxygenated PErfusion Versus Static Cold Storage of Human Donation After Cardiac Death Liver Transplants: An International-matched Case Analysis. Ann Surg. 2015;262(5):764-71.

8. Schlegel A, Kron P, De Oliveira ML, Clavien PA, Dutkowski P. Is single portal vein approach sufficient for hypothermic machine perfusion of DCD liver grafts? J Hepatol. 2016;64(1):239-41.

9. Ravikumar R, Jassem W, Mergental H, Heaton N, Mirza D, Perera MT, et al. Liver transplantation after ex vivo normothermic machine preservation: a Phase 1 (first-in-man) clinical trial. Am J Transplant. 2016; 16(6):1779-87.

10. op den Dries S, Karimian N, Sutton ME, Westerkamp AC, Nijsten MW, Gouw AS, et al. Ex vivo normothermic machine perfusion and viability testing of discarded human donor livers. Am J Transplant. 2013;13(5):1327-35.

11. Perera T, Mergental H, Stephenson B, Roll GR, Cilliers $H$, Liang $R$, et al. First human liver transplantation using a marginal allograft resuscitated by normothermic machine perfusion. Liver Transpl. 2016;22(1):120-4.

12. Watson CJE, Kosmoliaptsis V, Randle LV, Russell NK, Griffiths WJH, Davies S, et al. Preimplant Normothermic Liver Perfusion of a Suboptimal Liver Donated After Circulatory Death. Am J Transplant. 2016;16(1):353-7.

13. Reiling J, Lockwood DS, Simpson AH, Campbell CM, Bridle KR, Santrampurwala N, et al. Urea production during normothermic machine perfusion: Price of success? Liver Transpl. 2015;21(5): 700-3.

14. Vajdova K, Smrekova R, Kukan M, Lutterova M, Wsolova L. Bile analysis as a tool for assessing integrity of biliary epithelial cells after cold ischemia--reperfusion of rat livers. Cryobiology. 2000;41(2):145-52.

15. Hansen T, Hollemann D, Pitton MB, Heise M, Hoppe-Lotichius M, Schuchmann M, et al. Histological examination and evaluation of donor bile ducts received during orthotopic liver transplantation--a morphological clue to ischemic-type biliary lesion? Virchows Archiv. 2012;461(1):41-8.

16. op den Dries S, Westerkamp AC, Karimian N, Gouw AS, Bruinsma BG, Markmann JF, et al. Injury to peribiliary glands and vascular plexus before liver transplantation predicts formation of nonanastomotic biliary strictures. J Hepatol. 2014;60(6):1172-9.

17. Takakuwa Y, Kokai Y, Sasaki K, Chiba H, Tobioka H, Mori M, et al. Bile canalicular barrier function and expression of tight-junctional molecules in rat hepatocytes during common bile duct ligation. Cell Tissue Res. 2002;307(2):181-9.

18. Orman ES, Barritt ASt, Wheeler SB, Hayashi PH. Declining liver utilization for transplantation in the United States and the impact of donation after cardiac death. Liver Transpl. 2013;19(1):59-68. 
19. Blok JJ, Detry O, Putter H, Rogiers X, Porte RJ, van Hoek B, et al. Longterm results of liver transplantation from donation after circulatory death. Liver Transpl. 2016;22(8):1107-14.

20. Sutton ME, Op Den Dries S, Karimian N, De Boer MT, Wiersema-Buist J, Gouw ASH, et al. Criteria for viability assessment of discarded human donor livers during ex-vivo normothermic machine perfusion. Liver Transplant. 2014;20:S240.

21. Mergental H, Perera MT, Laing RW, Muiesan P, Isaac JR, Smith A, et al. Transplantation of declined liver allografts following normothermic ex-situ evaluation. Am J Transplant. 2016; 16(11):3235-45.

22. Imber CJ, St Peter SD, de Cenarruzabeitia IL, Lemonde H, Rees M, Butler A, et al. Optimisation of bile production during normothermic preservation of porcine livers. Am J Transplant. 2002;2(7):593-9.

23. Steffen JM. Glucose, glycogen, and insulin responses in the hypothermic rat. Cryobiology. 1988;25(2):94-101.

24. Brunner SM, Junger $H$, Ruemmele $P$, Schnitzbauer AA, Doenecke A, Kirchner GI, et al. Bile duct damage after cold storage of deceased donor livers predicts biliary complications after liver transplantation. J Hepatol. 2013;58(6):1133-9.

25. Karimian N, Weeder PD, Bomfati F, Gouw AS, Porte RJ. Preservation injury of the distal extrahepatic bile duct of donor livers is representative for injury of the intrahepatic bile ducts. J Hepatol. 2015;63(1):284-7.

26. Liu Q, Nassar A, Farias K, Buccini L, Baldwin W, Mangino M, et al. Sanguineous normothermic machine perfusion improves hemodynamics and biliary epithelial regeneration in donation after cardiac death porcine livers. Liver Transpl. 2014;20(8):987-99.

27. Op den Dries S, Karimian N, Westerkamp AC, Sutton ME, Kuipers M, Wiersema-Buist J, et al. Normothermic machine perfusion reduces bile duct injury and improves biliary epithelial function in rat donor livers. Liver Transpl. 2016;22(7):994-1005.

28. Fickert $\mathrm{P}$, Fuchsbichler A, Wagner $\mathrm{M}$, Zollner $\mathrm{G}$, Kaser $\mathrm{A}$, Tilg $\mathrm{H}$, et al. Regurgitation of bile acids from leaky bile ducts causes sclerosing cholangitis in Mdr2 (Abcb4) knockout mice. Gastroenterology. 2004;127(1):261-74.

29. Lowe PJ, Kan KS, Barnwell SG, Sharma RK, Coleman R. Transcytosis and paracellular movements of horseradish peroxidase across liver parenchymal tissue from blood to bile. Effects of alphanaphthylisothiocyanate and colchicine. Biochem J. 1985;229(2):529-37. 


\section{Supplementary material}

\section{Table S8.1 Characteristics of donors not used for NMP ( $N=5)$.}

\begin{tabular}{lc}
\hline Age (Years) & $43(30-50)$ \\
Gender (Male) & $2(40 \%)$ \\
BMI (Kg/m $\left.{ }^{2}\right)$ & $22(21-33)$ \\
Type (DCD) & $2(40 \%)$ \\
Cause of death & \\
Stroke & $4(80 \%)$ \\
Hypoxic brain injury & $1(20 \%)$ \\
Other & $0(0 \%)$ \\
Reason declined & \\
DCD + age & $1(20 \%)$ \\
DCD and logistics & $2(40 \%)$ \\
Steatosis/NASH & $2(40 \%)$ \\
ALT (U/L) & $4213-101)$ \\
WIT (min)* & 14,16 \\
\hline
\end{tabular}

* Only two DCD livers included, Abbreviations: BMI: body mass index, DCD: donation after circulatory death, NASH: non-alcohol steatohepatitis, ALT: alanine transaminase, WIT: warm ischaemic time. 


\section{Chapter 9}

General discussion 


\section{General discussion}

Liver transplantation is the preferred treatment option for patients with end stage liver disease. Unfortunately, an insufficient number of donor livers are available to transplant every patient on the waiting list; annually up to $10 \%$ of patients are delisted or die due to disease progression while waiting for a lifesaving liver transplant. ${ }^{1}$ More marginal organ donors, such as those donated after circulatory death (DCD) are being considered for transplantation in order to close the gap between supply and demand. Unfortunately, their use has been hampered by inferior graft performance and the development of biliary strictures in a significant proportion of recipients. ${ }^{2-4}$ This thesis aimed to identify factors responsible for the development of biliary injury using an animal model of partial hepatic ischaemia-reperfusion. Furthermore, the technique of normothermic machine perfusion of human donor livers was established and used as a platform to study the potential items involved in the pathophysiology of bile ducts injury in DCD livers.

In this chapter, the main findings of this thesis will be discussed and the implications for clinical practice assessed. Lastly, the conclusions of this thesis will be provided as well as future research opportunities.

\section{DCD donors are an underutilised resource in Australia}

Over the last several years there has been an initiative by the Australian Commonwealth Government to increase the number of organs available for transplant. In order to achieve this goal, the use of DCD organ donors was encouraged. In our experience, DCD donors are however often unsuitable liver donors. In chapter $\mathbf{2}$ we reviewed the characteristics of all Australian liver donors over a 10-year period to determine the impact of DCD organ donation on liver transplantation rates.

Although the number of organ donors and the number of livers donors substantially increased over the study period, a greater proportion of livers was deemed unsuitable for transplantation. Use rates of DBD donor livers remained stable around $20-25 \%$. DCD donor livers on the other hand were only rarely used for transplantation and as the number of DCD donors increased, the overall liver utilisation rates declined from $74 \%$ in 2005 to $57 \%$ in 2014.

The likely reasons for the high non-utilisation rates of DCD donor livers are the inferior long-term graft survival rates, as well as the increased risk of ischaemic type biliary strictures (ITBS) compared to livers from DBD donors. ${ }^{2,3}$ To facilitate the use of these high-risk donors in Australia, a better understanding is required of the pathophysiology of ITBS. This was addressed in Part I of this thesis. Another way to safely use DCD livers may be by applying normothermic machine perfusion (NMP) as a 
preservation method. This technique also allows for organ function assessment prior to transplantation, thereby avoiding the transplantation of non-viable liver grafts. The application of this technique was further addressed in part II of this thesis.

\section{Part I. biliary injury}

The development of an animal model of biliary injury and stricture formation

So far, much of what is known about the pathophysiology of biliary strictures originates from patient cohort studies. Prolonged ischaemia, as well as immunological factors and bile toxicity have been implicated in disease development., ${ }^{5,6}$ order to study the sequence of events responsible for the development of biliary injury and stricture formation we used an animal model of partial hepatic ischaemia-reperfusion (Chapters $\mathbf{3}$ and $\mathbf{4}$ ). This model was considered less technically challenging than a liver transplantation model and we expected it to provide more reproducible results within a short timeframe. Within this model we tested the role of ischaemia as well as lipopolysaccharides (LPS) in the development of biliary injury. Animals were exposed to warm hepatic ischaemia and reperfusion (IRI), administration of lipopolysaccharides or a combination of both IRI and LPS and bile liver and bile duct tissue was collected.

As the study described in Chapter 3 only presents short-term effects of both IRI and LPS, further studies need to be conducted to confirm stricture formation does occur over time. Filling of the biliary tree with a methacrylate polymer mixture to obtain a bile duct cast or cholangiography could be used to confirm the presence of biliary strictures. $^{7,8}$

The role of ischaemia in the development of biliary injury and stricture formation

Cholangiocytes are more sensitive to IRI than hepatocytes. ${ }^{9}$ Upon reperfusion, they produce more reactive oxygen species, which combined with a lower basal level of anti-oxidants lead to a higher rate of cell death. Although these experiments described by Noack et al. were conducted using cultured cells, there is convincing evidence from clinical scenarios and studies to confirm these findings. Firstly, the blood supply to the biliary tree is mainly arterial and patients with hepatic artery thrombosis following transplantation develop biliary necrosis and stricture formation. ${ }^{10,11}$ Furthermore, extended periods of warm ischaemia prior to organ retrieval were found to be associated with the development of ischaemic type biliary strictures following transplantation ${ }^{12,13}$ In addition, a warm ischaemic period 
exceeding 25 minutes was associated with an inferior graft survival rate of DCD grafts. $^{2}$

Although it seems clear that ischaemia is a risk factor, we did not find any evidence of biliary injury following $\mathbf{3 0}$ minutes of warm ischaemia in our rat model as described in Chapter 3. This may have been due to the short duration of blood flow interruption to the liver. Longer periods of warm ischaemia have previously been used in animal models of IRI and is well tolerated by rats. ${ }^{14}$ Our rationale for using the 30 minute cut off however was that this is currently the maximum duration of ischaemia acceptable for the use of DCD grafts.

Another explanation could be that rats respond in a different fashion to warm ischaemia than humans. Op den Dries et al. previously conducted an experiment where rat livers were exposed to 30 minutes of warm ischaemia and 3 hours of cold storage. ${ }^{15}$ Following 2 hours of reperfusion, they did not find any evidence of biliary injury using routine histological assessment. When transmission electron microscopy was performed however, the number of mitochondria in cholangiocytes was markedly reduced, microvilli were lost and contact between neighbouring cholangiocytes was reduced.

Although we have successfully established a rat model of biliary injury, the development of ischaemia-induced biliary injury could not be determined using routine histological assessment. As ischaemia is considered an important risk factor for the development of ITBS following transplantation, the use of a different species such as pigs should be considered for future animal studies.

\section{Endotoxins as a mediator of biliary injury}

Endotoxins, in the form of LPS, can substantially enhance hepatic IRI resulting in more severe necrosis compared to LPS or IRI alone. ${ }^{14}$ In a transplantation setting, the deleterious effects of endotoxin exposure in the donor can be transferred to the recipient resulting in inferior graft function. ${ }^{16,17}$ Zipfel et al. reported that livers of donors that had plasma LPS concentrations over $12 \mathrm{pg} / \mathrm{ml}$ failed in $75 \%$ of cases within the first two years of transplant compared to no graft failures in the group with LPS levels $<12 \mathrm{pg} / \mathrm{ml}^{18}$ As the above-mentioned studies only focussed on hepatocellular function and injury, the study described in Chapter 3. aimed to determine the effects of LPS on cholangiocytes. In this study we administered $1 \mathrm{mg} / \mathrm{kg}$ of LPS. This is a relatively low dose compared to the $15-30 \mathrm{mg} / \mathrm{kg}$ LPS used in sepsis studies. ${ }^{19}$ Despite the low dosage used, small cholangiocytes in the portal triads were severely damaged following six hours of exposure to LPS with most or all ducts infiltrated by inflammatory cells, severe degenerative changes and ductular proliferation. We however did not find any evidence of injury to the large intrahepatic or common bile duct. One possible explanation might be that 6 hours is too early for large bile duct damage to occur. Histological scoring of the large ducts did indicate 
some early changes with atypical large bile duct cells, but not to the extent of the injury we saw in small bile ducts.

Although at this stage it remains unclear if LPS-mediated injury would progress to stricture formation, previous studies have linked immunological factors to the onset of ITBS in transplant recipients. ${ }^{5,6}$ Chemokine receptor polymorphisms resulting in loss of function, cytomegalovirus infection as well as transplantation across blood groups have all been implicated in late ITBS development, occurring more often in the periphery of the liver. In addition, Friedrich et al. recently identified the crucial role of CD14/TLR-4 signalling in ITBS formation. ${ }^{20}$ As this pathway becomes activated in response to LPS, it possibly implicates a role for LPS-mediated injury in ITBS formation.

The role of macrophages in the development of LPS-induced biliary injury

Kupffer cells, the resident macrophages of the liver, become activated upon stimulation with pathogen-associated molecular patterns (PAMP) such as LPS and release reactive oxygen species and cytokines in response. ${ }^{21}$ This leads to the recruitment of neutrophils, which can eventually results in cellular injury. ${ }^{14}$ Hepatic depletion of macrophages has previously been shown to have a protective effect on the development of IRI and endotoxin-induced hepatocellular injury. ${ }^{16,22}$ We therefore aimed to determine the role of macrophages in the development of LPS-induced biliary injury in Chapter 4. Macrophages were successfully depleted from the liver using clodronate liposomes and rats were exposed to LPS or IRI+LPS for 6 hours. Histological assessment of small bile duct injury revealed that severe biliary injury persisted in the absence of macrophages. These data suggest that cholangiocytes are directly affected by LPS. Cholangiocytes are exposed to endotoxins as they are excreted in bile following their removal from the circulation. ${ }^{23}$ As this process is not altered by macrophage depletion, it could well be the reason for the development of biliary injury in the absence of macrophages. Furthermore, cholangiocytes play an active role in innate immunity as they release cytokines upon stimulation. ${ }^{24}$ Assessment of biliary concentration of cytokines in our study revealed that the concentrations of monocyte chemo-attractant protein-1 (Mcp-1) and tissue inhibitor of metalloproteinase-1 (Timp-1) were most significantly increased following LPS administration. Macrophage depletion did not alter this local inflammatory environment, which could explain the persistent biliary injury. Ultimately, specific assessment of cholangiocytes, by means of cell sorting, is required to determine the exact mechanism by which LPS induces biliary injury. 


\section{Blood biliary barrier assessment and involvement in LPS-mediated biliary injury}

Epithelial barrier function is often tested in vitro using non-metabolised sugars such as inulin or mannitol. ${ }^{25}$ Furthermore trans-epithelial electrical resistance is assessed to determine the permeability of the epithelial barrier. ${ }^{26}$ The blood-biliary barrier (BBB) has not frequently been assessed in vivo. Horseradish peroxidase (HRP) as well as fluorescence labelled UDCA were used in previous animal studies. ${ }^{27-29}$ As HRP is readily available, repeated measures can be performed and leakage across the barrier can be assessed without the need to obtain cryosections, we decided to use HRP in our experiments described in Chapter $\mathbf{3}$ and 4. Following intravenous injection, HRP appears in bile via two distinct routes, the para-cellular route (across tight junctions), and trans-cellular transport (30). The administration of LPS increased the amount of HRP that leaked across the BBB by the para-cellular route. Immunofluorescence staining for tight junction protein zonula occludens-1, showed a severely disturbed staining pattern in animals that developed biliary injury. Whether impaired BBB function leads to the development of a specific biliary injury (as bile is retained in the portal tract area) or if it is merely the result of severe systemic insult remains to be determined.

Comparable inflammatory propensity of portal blood obtained from DCD donors to that of DBD donors

As LPS was found to be a potent inducer of biliary injury in our rat model, we aimed to determine whether endotoxaemia occurs during organ retrieval of DCD donors in humans (Chapter 5). DCD organ donation takes place following the withdrawal of life support and cessation of circulation. Throughout this process, hypoxia and hypoperfusion of organs such as the gut occurs which could result in endotoxaemia. ${ }^{31}$ Previous studies had shown that blood collected during DBD donation contained increased concentrations of endotoxins, especially at the time of hilar dissection. ${ }^{32}$ However, no data were available on the occurrence of endotoxaemia during the DCD donation process. We therefore collected portal venous blood samples from DBD and DCD donors throughout the organ retrieval process and assessed the concentration of endotoxins both directly and indirectly. We performed the conventional Limulus amebocyte lysate ( $L A L)$ test on a subset of samples to determine the content of endotoxins. Additionally, we performed an NFkB-cell based assay, which measures the inflammatory response mediated by all pro-inflammatory factors present in the tested samples such as damage-associated molecular patterns and endotoxin.

As opposed to what we anticipated, no differences were found in the concentration of endotoxins or the propensity of portal samples to induce an inflammatory response between DBD and DCD donors. A possible contributing factor could have been the 
timing of sample collection in this study. It was often difficult to obtain a portal venous sample at the start of cold perfusion in DCD donors. At the time of sample collection, cold perfusate might have already washed out the inflammatory mediators from the portal system. It however needs to be noted that even portal samples collected directly following the initiation of cold perfusion failed to induce an inflammatory response.

Up until this point it has been unclear to what extent endotoxaemia contributed to the development of IRI in DCD transplantation. Although inflammatory mediators were not present in blood flushing through the liver, hepatic macrophage activation was enhanced in DCD donors at the time of organ retrieval. This increase in macrophage activation is likely the result of exposure to local damage-associated molecular patterns (DAMPs) released by the liver upon ischaemic injury.

In addition to the blood samples, bile and common bile duct (CBD) sections were collected to assess the degree of bile duct damage at the time of organ retrieval. When systematically assessing CBD sections of DCD donors, only mild injury was observed to all components apart from the peribiliary glands, which were severely damaged. In addition to CBD histology, lactate dehydrogenase (LDH) in bile was measured as a biomarker of biliary injury. This likely represents damage to the entire biliary tree and LDH levels were significantly higher in bile collected from DCD donors compared to DBD donors. Although we were not able to determine if the sustained biliary injury ultimately resulted in stricture formation, results from this study might indicate the need for early intervention in order to prevent the development of ITBS.

\section{Part II: Machine perfusion}

The second half of this thesis focussed on the application of machine perfusion to safely increase the use of DCD donor livers for transplantation. This setup was furthermore used as a platform to assess the bile ducts of DCD livers prior to transplantation. The safety and feasibility of different perfusion setups used around the world was reviewed and a normothermic perfusion protocol was subsequently developed at the Princess Alexandra Hospital in Brisbane.

Systematic review of different perfusion modalities used across the globe

A systematic review of the literature identified 22 manuscripts describing the use of machine perfusion techniques to perfuse liver grafts from marginal donors (Chapter 6). Of those we identified nine studies that described hypothermic perfusion (HMP, $4-10^{\circ} \mathrm{C}^{33-41}$ ), four described mid-thermic perfusion (MMP, $13-24^{\circ} \mathrm{C}^{42-45}$ ) and nine discussed normothermic protocols (NMP, $37^{\circ} \mathrm{C}^{46-54}$ ). Each centre considered their 
protocol technically feasible and no major technical complications were encountered that might led to graft loss or exclusion from the study. Compared to NMP, HMP is rather uncomplicated, as no oxygen carrier is needed at this low temperature. Furthermore, static cold storage is the default backup in case of pump failure. NMP on the other hand, requires extensive knowledge of multiple aspects of liver metabolism and pump failure immediately exposes the livers to a warm ischaemic insult.

When it comes to biliary complications, oxygenated HMP of DCD donor livers significantly reduced the incidence of ITBS compared to non-perfused controls zero versus $11(22 \%), p=0.015)$ (34). NMP only just entered the clinical trials phase and no results are available yet on the influence on ITBS formation. It was however the only technique that allowed for viability testing prior to transplantation. Both lactate clearance as well as bile production have been proposed as indicators of graft viability however results from large randomised trials are awaited to determine if these factors truly predict graft function in short or long term. ${ }^{50,52}$ Nevertheless, we aimed to establish a NMP protocol at our centre as we believe that viability testing is one of the most important features of the application of machine perfusion.

The establishment of a normothermic machine perfusion protocol at the Princess Alexandra Hospital

Encouraged by the favourable results published by op den Dries et al., and later the Oxford group led by Professor Friend, we decided to develop a NMP protocol at our centre $(46,49)$. The circuit was custom made using locally available resources. A single centrifugal pump perfused the hepatic artery directly whereas the portal vein was gravity fed via a reservoir. During the perfusion period, taurocholic acid, amino acids, insulin and prostacyclin were continuously infused and blood collected from the donor was used as perfusate. Finally, a scavenger system was in place to pump leaked perfusion solution back into the closed circuit. In this thesis, the results of ten consecutive perfusions are discussed in Chapter 7 and 8. In our experience, it was relatively straightforward to establish perfusion and only minor adjustments had to be made to the pump settings throughout the perfusion period to maintain near physiological pressures and flows. As the metabolic functions of the liver are diverse and complex, we would like to discuss some different aspects separately.

\section{Viability assessment during NMP}

Lactate clearance has been proposed as a way to distinguish the "good livers" from the "bad". In the landmark papers by Perera et al. and Watson et al. describing the first transplants of declined marginal DCD livers following NMP, lactate clearance was used as one of the major determinants of viability. ${ }^{50,53}$ In a later report by the Birmingham group, a more comprehensive assessment protocol was presented which 
besides lactate clearance included two out of the three following factors: macroscopic appearance, $\mathrm{pH}$ and arterial and portal flow. ${ }^{55}$ Bile production was only used to determine viability if a graft failed to clear lactate.

When we applied the lactate clearance cut-off to our cohort, seven livers were deemed viable and three were deemed non-viable. During the perfusions, other parameters associated with liver function such as potassium concentrations, $\gamma$-glutamyl transferase and AST correlated well with lactate clearance. At the end of perfusion, hepatocellular architecture was preserved and no evidence of necrosis was present upon histological assessment. We therefore concluded that lactate clearance was a good determinant of viability from a hepatocellular aspect.

\section{Glucose metabolism of DCD donor livers during NMP}

Throughout the perfusion period we observed a distinct pattern in the glucose levels of DCD compared to DBD livers. Within the first hour of perfusion of DCD grafts, glucose concentrations increased despite the administration of $100 \mathrm{IU}$ of insulin. During the subsequent hours of perfusion, glucose levels decreased to below baseline. These findings are consistent with the current literature $(53,56)$ and glycogenolysis is the most likely explanation. ${ }^{57}$ Especially as venous glucose concentrations during this period were higher than arterial glucose concentration. Ultimately, glycogen stores are restored at the end of NMP as was shown by Megenthal et al.. ${ }^{55}$

Glucose concentrations at the start of each perfusion were largely dependent on the type of anticoagulant (citrate-based anticoagulation solution or heparin) used to store donor blood. Furthermore, during the first two perfusions we did not use a haemofilter and a $5 \%$-glucose solution was used to counteract the sodium increase resulting from the administration of sodium bicarbonate. This, in combination with the glycogenolysis of the DCD grafts resulted in peak glucose concentrations of 32.6 (20.9-34.5) $\mathrm{mmol} / \mathrm{L}$. As glucose is a major component of the calculated osmolality, this resulted in osmolality levels well above the physiological threshold of $295 \mathrm{mmol} / \mathrm{L}$. The use of a dialysis machine prior to, or during, machine perfusion might therefore be considered.

\section{Urea production during NMP}

Although we initially regarded urea production as a good indicator of synthetic liver function, its effect on osmolality of the perfusate meant that urea built-up in the closed system might need to be avoided when prolonged perfusion is required. One possible way of doing so is the use of a dialysis system as was employed by Banan et al.. ${ }^{58} \mathrm{~A}$ major disadvantage of the use of a dialysis system within the first 2 hours of perfusion is that it might mask the production of lactate, potassium and other small 
proteins that indicate organ dysfunction. Although dialysis is unlikely to counteract the ongoing production of lactate by non-viable grafts, it might mean that the cut-off of $2 \mathrm{mmol} / \mathrm{L}$ becomes jeopardised.

\section{Assessment of biliary viability during machine perfusion}

To determine the effect of NMP on the bile duct integrity we collected samples of the CBD at the time of organ donation, end of cold storage and end of perfusion. Unfortunately, NMP was not able to prevent biliary injury and the majority of CBD sections showed evidence of severe injury, regardless of metabolic performance.

In addition to the role of NMP, we determined if the degree of injury to the CBD correlated with the severity of injury to the intrahepatic ducts. In total, 15 livers deemed unsuitable for transplantation were included in the comparison discussed in chapter 8. Compared to the CBD, significantly less biliary injury was observed at the level of the main intrahepatic ducts as well as segmental ducts. These findings are in stark contrast to what has previously been reported by Karimian et al.. ${ }^{59}$ The livers included in their study were however not re-perfused and therefore the overall degree of injury was much less compared to what we observed in our cohort. When a sub-analysis was performed including those livers that did not undergo NMP in our cohort, the degree of injury to the CBD was much less in these unperfused grafts and correlated well with the degree of injury to main ducts or the segmental ducts. It might therefore not be possible to rely on the histological assessment of CBD injury to determine biliary viability, and a more reliable biomarker is needed.

We therefore measured the levels of LDH in bile as has previously been used as a biomarker of biliary injury. Immediately following re-perfusion of the graft, LDH levels in bile were significantly higher than those measured in the donor, correlating with a higher degree of injury observed upon histological assessment. At the end of the perfusion period however, LDH levels reduced by 1.6 fold although significant histological damage was present. This might indicate that by that time, no more LDH is released as the majority of cholangiocytes have died. Therefore caution has to be taken when interpreting LDH results without the support of histological evidence.

\section{Assessment of the blood-biliary-barrier during NMP}

As we previously identified impairment of the BBB as a possible contributor to the development of biliary injury in Chapter 3, we aimed to determine its integrity during NMP in Chapter 8. In line with the rat studies, we administered HRP to the circuit and measured the output of HRP in bile during the perfusion of two livers. We were able to measure the concentrations of HRP in bile however the interpretation of the results is complicated by several factors. The liver needs to produce sufficient amounts of bile 
to allow for the assay to be conducted. Furthermore, as extension tubing or paediatric feeding tubes are used to facilitate bile collection outside the main sterile field, the concentration of bile collected might reflect what has been excreted several minutes earlier. Therefore trans-cytosis rather than para-cellular movements could accidentally be assessed. In future experiments, a different approach to assessment of the BBB should be considered

\section{Conclusion}

This thesis assessed ways to improve the use and outcome of DCD liver transplantation. Different aspects of the pathophysiology of bile duct injury following IRI and transplantation were assessed and endotoxins were shown to be potent mediators of bile duct injury. This acute injury was not mediated by macrophages but evidence suggested that a local inflammatory milieu surrounding the bile ducts more likely played a role. Although endotoxins were identified as potent mediators of biliary injury, such factors were not observed during the DCD donation process.

Another way to facilitate the safe use of DCD donor livers is by machine perfusion. In our unit at the Princess Alexandra Hospital in Brisbane, we successfully established a normothermic perfusion protocol and were able to use this technique to assess suitability for transplantation. Viability of the bile ducts is however critical to reduce the incidence of ITBS and further work is needed to find biomarkers that predict viability.

\section{Future perspectives}

Macrophages were shown not to play a role in the development of LPS-mediated biliary injury. Future work should therefore focus on the direct effect LPS has on cholangiocytes. A proposed way to do this is to obtain tissue from the biliary tree at the end of the experiment and assess gene expression patterns of cholangiocytes using RTqPCR.

As biliary cytokine concentrations were still present following macrophage depletion, it would be interesting to test TLR-4 or Tnf- $\alpha$ receptor agonists as a therapeutic option. We have performed some preliminary experiments using TACE inhibitor TIMP3. This protein prevents the cleavage of pro-TNF-alpha into its active soluble form and pre-treatment with TIMP-3 protected the animals from the LPS-induced biliary injury. As was highlighted at the beginning of this discussion, rats respond differently to ischaemic injury than humans and might therefore not be the best animals to assess biliary injury in. Alternatively, pig models should be considered for future 
experiments. Experiments in these animals have shown similar biliary injury patterns and due to the size of the animal, machine perfusion or transplant experiments could be considered.

Viability of the bile ducts is an important factor determining long-term graft survival of DCD donor livers. Future research should be directed to determine a suitable biomarker of biliary viability during NMP.

We have shown that NMP of human donor livers is feasible and that viability can be assessed. Ultimately, we would like move toward a setting where livers deemed viable by NMP are used for transplantation. 


\section{References}

1. ANZLTR. 24th annual report Australia and New Zealand liver transplant registry. 2014.

2. Blok JJ, Detry O, Putter H, Rogiers X, Porte RJ, van Hoek B, et al. Longterm results of liver transplantation from donation after circulatory death. Liver Transpl. 2016;22(8):1107-14.

3. Dubbeld J, Hoekstra H, Farid W, Ringers J, Porte RJ, Metselaar HJ, et al. Similar liver transplantation survival with selected cardiac death donors and brain death donors. Br J Surg. 2010;97(5):744-53.

4. Laing RW, Scalera I, Isaac J, Mergental H, Mirza DF, Hodson J, et al. Liver transplantation using grafts from donors after circulatory death: A propensity-matched study from a single centre. Am J Transplant. 2016.

5. Op den Dries S, Sutton ME, Lisman T, Porte RJ. Protection of bile ducts in liver transplantation: looking beyond ischemia. Transplantation. 2011;92(4):373-9.

6. op den Dries S, Buis $\mathrm{Cl}$, Adelmeijer J, Van der Jagt EJ, Haagsma EB, Lisman T, et al. The combination of primary sclerosing cholangitis and CCR5-Delta32 in recipients is strongly associated with the development of nonanastomotic biliary strictures after liver transplantation. Liver Int. 2011;31(8): 1102-9.

7. Fickert P, Zollner G, Fuchsbichler A, Stumptner C, Weiglein AH, Lammert F, et al. Ursodeoxycholic acid aggravates bile infarcts in bile duct-ligated and Mdr2 knockout mice via disruption of cholangioles. Gastroenterology. 2002;123(4):1238-51.

8. Goetz M, Lehr HA, Neurath MF, Galle PR, Orth T. Long-term evaluation of a rat model of chronic cholangitis resembling human primary sclerosing cholangitis. Scand J Immunol. 2003;58(5):533-40.

9. Noack K, Bronk SF, Kato A, Gores GJ. The greater vulnerability of bile duct cells to reoxygenation injury than to anoxia. Implications for the pathogenesis of biliary strictures after liver transplantation. Transplantation. 1993;56(3):495-500.

10. J.M.A. Northover JT. a new look at the arterial supply of the bile duct in man and its surgical implications. Br J Surg. 1979;66:379-84.

11. Zajko AB, Campbell WL, Logsdon GA, Bron KM, Tzakis A, Esquivel CO, et al. Cholangiographic findings in hepatic artery occlusion after liver transplantation. AJR Am J Roentgenol. 1987;149(3):485-9.

12. Taner CB, Bulatao IG, Willingham DL, Perry DK, Sibulesky L, Pungpapong S, et al. Events in procurement as risk factors for ischemic cholangiopathy in liver transplantation using donation after cardiac death donors. Liver Transpl. 2012;18(1):100-11.

13. de Vera ME, Lopez-Solis R, Dvorchik I, Campos S, Morris W, Demetris AJ, et al. Liver transplantation using donation after cardiac death donors: long-term follow-up from a single center. Am J Transplant. 2009;9(4):773-81.

14. Caraceni P, Pertosa AM, Giannone F, Domenicali M, Grattagliano I, Principe A, et al. Antagonism of the cannabinoid $C B-1$ receptor protects rat liver against ischaemia-reperfusion injury complicated by endotoxaemia. Gut. 2009;58(8):1135-43.

15. Op den Dries S, Karimian N, Westerkamp AC, Sutton ME, Kuipers M, Wiersema-Buist J, et al. Normothermic machine perfusion reduces bile duct injury and improves biliary epithelial function in rat donor livers. Liver Transpl. 2016;22(7):994-1005.

16. Vajdova K, Smrekova R, Kukan M, Jakubovsky J, van Rooijen N, Horecky J, et al. Endotoxin-induced aggravation of preservation-reperfusion injury of rat liver and its modulation. $\mathrm{J}$ Hepatol. 2000;32(1):112-20.

17. Azoulay D, Astarcioglu I, Lemoine A, Dennison A, Mathieu D, Saulnier C, et al. The effects of donor and recipient endotoxaemia on TNF alpha production and mortality in the rat model of syngenic orthotopic liver transplantation. Transplantation. 1995;59(6):825-9.

18. Zipfel A, Schenk M, You MS, Lauchart W, Bode C, Viebahn R. Endotoxaemia in organ donors: graft function following liver transplantation. Transpl Int. 2000;13 Suppl 1:S286-7.

19. Recknagel P, Gonnert FA, Halilbasic E, Gajda M, Jbeily N, Lupp A, et al. Mechanisms and functional consequences of liver failure substantially differ between endotoxaemia and faecal peritonitis in rats. Liver Int. 2013;33(2):283-93. 
20. Friedrich K, Smit M, Brune M, Giese T, Rupp C, Wannhoff A, et al. CD14 is associated with biliary stricture formation. Hepatology. 2016.

21. Kapoor S. Hepatic ischaemia-reperfusion injury from bench to bedside ( $\mathrm{Br} J$ Surg 2010; 97: 14611475). Br J Surg. 2011;98(3):459; author reply -60.

22. von Frankenberg M, Golling M, Mehrabi A, Nentwich H, Klar E, Kraus TW. Donor pretreatment with gadolinium chloride improves early graft function and survival after porcine liver transplantation. Transpl Int. 2003;16(11):806-13.

23. Freudenberg MA, Galanos C. Bacterial lipopolysaccharides: structure, metabolism and mechanisms of action. International reviews of immunology. 1990;6(4):207-21.

24. Syal G, Fausther M, Dranoff JA. Advances in cholangiocyte immunobiology. Am J Physiol Gastrointest Liver Physiol. 2012;303(10):G1077-86.

25. Guntaka SR, Samak G, Seth A, LaRusso NF, Rao R. Epidermal growth factor protects the apical junctional complexes from hydrogen peroxide in bile duct epithelium. Lab Invest. 2011;91(9):1396409.

26. Hanada S, Harada M, Koga H, Kawaguchi T, Taniguchi E, Kumashiro R, et al. Tumor necrosis factoralpha and interferon-gamma directly impair epithelial barrier function in cultured mouse cholangiocytes. Liver Int. 2003;23(1):3-11.

27. Fickert $\mathrm{P}$, Fuchsbichler A, Wagner $\mathrm{M}$, Zollner $\mathrm{G}$, Kaser $\mathrm{A}$, Tilg $\mathrm{H}$, et al. Regurgitation of bile acids from leaky bile ducts causes sclerosing cholangitis in Mdr2 (Abcb4) knockout mice. Gastroenterology. 2004;127(1):261-74.

28. Takakuwa Y, Kokai Y, Sasaki K, Chiba H, Tobioka H, Mori M, et al. Bile canalicular barrier function and expression of tight-junctional molecules in rat hepatocytes during common bile duct ligation. Cell Tissue Res. 2002;307(2):181-9.

29. S. MEPDCPC. role of IL-10 in hepatocyte tight junction alteration in mouse model of experimental cholitis. Mol Med. 2002;8(7).

30. Lowe PJ, Kan KS, Barnwell SG, Sharma RK, Coleman R. Transcytosis and paracellular movements of horseradish peroxidase across liver parenchymal tissue from blood to bile. Effects of alphanaphthylisothiocyanate and colchicine. Biochem J. 1985;229(2):529-37.

31. Sun $\mathrm{Y}$, Hardaway RM, Williams $\mathrm{CH}$. Comparison of bacterial translocation during traumatic shock and hemorrhagic shock in rats. Int Surg. 1997;82(2):134-6.

32. Pillay SP, Moore TD, Lynch SV, Whiting RF, Balderson GA, Strong RW. Endotoxin levels in adult liver donors. Aust N Z J Surg. 1994;64(9):615-7.

33. Guarrera JV, Henry SD, Samstein B, Reznik E, Musat C, Lukose TI, et al. Hypothermic machine preservation facilitates successful transplantation of "orphan" extended criteria donor livers. Am J Transplant. 2015;15(1):161-9.

34. Dutkowski P, Polak WG, Muiesan P, Schlegel A, Verhoeven CJ, Scalera I, et al. First Comparison of Hypothermic Oxygenated PErfusion Versus Static Cold Storage of Human Donation After Cardiac Death Liver Transplants: An International-matched Case Analysis. Ann Surg. 2015;262(5):764-71.

35. Dutkowski P, Schlegel A, de Oliveira M, Mullhaupt B, Neff F, Clavien PA. HOPE for human liver grafts obtained from donors after cardiac death. J Hepatol. 2014;60(4):765-72.

36. Jomaa A, Gurusamy K, Siriwardana PN, Claworthy I, Collier S, de Muylder P, et al. Does hypothermic machine perfusion of human donor livers affect risks of sinusoidal endothelial injury and microbial infection? A feasibility study assessing flow parameters, sterility, and sinusoidal endothelial ultrastructure. Transplant Proc. 2013;45(5):1677-83.

37. Monbaliu D, Liu Q, Libbrecht L, De Vos R, Vekemans K, Debbaut C, et al. Preserving the morphology and evaluating the quality of liver grafts by hypothermic machine perfusion: a proof-of-concept study using discarded human livers. Liver Transplant. 2012;18(12):1495-507.

38. Vekemans K, van Pelt J, Komuta M, Wylin T, Heedfeld V, Detry O, et al. Attempt to rescue discarded human liver grafts by end ischemic hypothermic oxygenated machine perfusion. Transplantation proceedings. 2011;43(9):3455-9.

39. Westerkamp AC, Karimian N, Matton APM, Mahboub P, van Rijn R, Wiersema-Buist J, et al. Oxygenated Hypothermic Machine Perfusion After Static Cold Storage Improves Hepatobiliary Function of Extended Criteria Donor Livers. Transplantation. 2016. 
40. Schlegel A, Kron P, De Oliveira ML, Clavien PA, Dutkowski P. Is single portal vein approach sufficient for hypothermic machine perfusion of DCD liver grafts? J Hepatol. 2016;64(1):239-41.

41. Guarrera JV, Estevez J, Boykin J, Boyce R, Rashid J, Sun S, et al. Hypothermic machine perfusion of liver grafts for transplantation: technical development in human discard and miniature swine models. Transplant Proc. 2005;37(1):323-5.

42. Bruinsma BG, Yeh H, Özer S, Martins PN, Farmer A, Wu W, et al. Subnormothermic machine perfusion for ex vivo preservation and recovery of the human liver for transplantation. Am J Transplant. 2014; 14(6):1400-9.

43. Bruinsma BG, Avruch JH, Weeder PD, Sridharan GV, Uygun BE, Karimian NG, et al. Functional human liver preservation and recovery by means of subnormothermic machine perfusion. J Vis Exp. 2015(98).

44. Bruinsma BG, Sridharan GV, Weeder PD, Avruch JH, Saeidi N, Ozer S, et al. Metabolic profiling during ex vivo machine perfusion of the human liver. Sci Rep. 2016;6:22415.

45. Hoyer DP, Luer B, Efferz P, Paul A, Minor T. Controlled oxygenated rewarming or normothermic machine perfusion for endischemic liver reconditioning before transplantation? Transplant Int. 2015;28:93.

46. Ravikumar R, Jassem W, Mergental H, Heaton N, Mirza D, Perera MTPR, et al. Liver Transplantation After Ex Vivo Normothermic Machine Preservation: A Phase 1 (First-in-Man) Clinical Trial. Am J Transplant. $2016 ; 16(6): 1779-87$.

47. Bellomo R, Marino B, Starkey G, Fink M, Wang BZ, Eastwood GM, et al. Extended normothermic extracorporeal perfusion of isolated human liver after warm ischaemia: a preliminary report. Crit Care Resusc. 2014;16(3):197-201.

48. Bellomo R, Marino B, Starkey G, Wang BZ, Fink MA, Zhu N, et al. Normothermic extracorporeal human liver perfusion following donation after cardiac death. Crit Care Resusc. 2013;15(2):78-82.

49. op den Dries S, Karimian N, Sutton ME, Westerkamp AC, Nijsten MWN, Gouw ASH, et al. Ex vivo normothermic machine perfusion and viability testing of discarded human donor livers. Am J Transplant. 2013;13(5):1327-35.

50. Perera T, Mergental H, Stephenson B, Roll GR, Cilliers $H$, Liang $R$, et al. First human liver transplantation using a marginal allograft resuscitated by normothermic machine perfusion. Liver Transpl. 2016;22(1):120-4.

51. Reiling J, Lockwood DSR, Simpson AH, Campbell CM, Bridle KR, Santrampurwala N, et al. Urea production during normothermic machine perfusion: Price of success? Liver Transplantation. 2015;21(5):700-3.

52. Sutton ME, op den Dries S, Karimian N, Weeder PD, de Boer MT, Wiersema-Buist J, et al. Criteria for viability assessment of discarded human donor livers during ex vivo normothermic machine perfusion. PloS One. 2014;9(11):e110642.

53. Watson CJE, Kosmoliaptsis V, Randle LV, Russell NK, Griffiths WJH, Davies S, et al. Preimplant Normothermic Liver Perfusion of a Suboptimal Liver Donated After Circulatory Death. Am J Transplant. 2016;16(1):353-7.

54. Karimian N, Matton APM, Westerkamp AC, Burlage LC, Op den Dries S, Leuvenink HGD, et al. Ex Situ Normothermic Machine Perfusion of Donor Livers. J Vis Exp. 2015(99):e52688.

55. Mergental H, Perera MT, Laing RW, Muiesan P, Isaac JR, Smith A, et al. Transplantation of Declined Liver Allografts Following Normothermic Ex-Situ Evaluation. Am J Transplant. 2016.

56. Imber CJ, St Peter SD, de Cenarruzabeitia IL, Lemonde H, Rees M, Butler A, et al. Optimisation of bile production during normothermic preservation of porcine livers. AmJ Transplant. 2002;2(7):593-9.

57. Steffen JM. Glucose, glycogen, and insulin responses in the hypothermic rat. Cryobiology. 1988;25(2):94-101.

58. Banan B, Watson R, Xu M, Lin Y, Chapman W. Development of a normothermic extracorporeal liver perfusion system toward improving viability and function of human extended criteria donor livers. Liver Transpl. 2016;22(7):979-93.

59. Karimian N, Weeder PD, Bomfati F, Gouw AS, Porte RJ. Preservation injury of the distal extrahepatic bile duct of donor livers is representative for injury of the intrahepatic bile ducts. J Hepatol. 2015;63(1):284-7. 
Summary 


\section{Summary}

Chapter 1 provides a general introduction to liver transplantation and the current clinical limitations as well as overview of the outline of this thesis. Currently more livers donated after circulatory death (DCD) are being considered for transplantation in order to increase the number of donor livers available for patients on the transplant waiting list. Unfortunately, the use of these grafts is often complicated by the formation of ischaemic type biliary strictures (ITBS). As a result, DCD liver recipients frequently undergo re-interventions and long-term graft survival is limited. The overarching aim of this thesis was to better understand the pathophysiology of biliary stricture formation in DCD donors. To achieve this we developed an animal model of biliary injury as well as an ex-vivo normothermic machine perfusion setup used to perfuse livers declined for transplantation.

In Chapter $\mathbf{2}$ the characteristics of all adult organ donors over the last decade were reviewed to determine the impact of DCD donation on liver transplantation rates in Australia. Since the implementation of DCD donation in 2008, their contribution to the overall number of organ donors steadily increased to $29 \%$ in 2014 . Unfortunately DCD grafts were used only in a small proportion of cases, which contributed to an increase in the number of livers declined for transplantation (42 (26\%) in 2005 versus 141 (43\%) in 2014). Furthermore, multivariable regression identified DCD donor type as the most important independent risk factor for liver non-use (OR $24.06(95 \% \mathrm{Cl}$ 17.47-33.14)) followed by advanced donor age, obesity, and diabetes. The limited use of DCD donors is likely the result of the unacceptably high risk of ITBS formation. A better understanding of the pathophysiology of these strictures is required to ultimately facilitate the use of more DCD grafts. Another possible approach could be the use of machine perfusion as an alternative preservation method. Additionally, modifiable risk factors such as the period of warm and cold ischaemic time should be kept to a minimum. Characterisation of DCD grafts declined for transplantation in the state of Queensland identified 33 (41\%) donors who had zero, one or two marginal donor criteria that could be likely candidates for the application of machine perfusion.

Chapter 3 focussed on pathophysiology of biliary injury. In this study, the role of lipopolysaccharides (LPS) and ischaemia-reperfusion was assessed. Rats were exposed to $1 \mathrm{mg} / \mathrm{kg}$ of LPS or 30 minutes of warm hepatic ischaemia to $70 \%$ the liver. The animals were subsequently allowed to recover for one or six hours after which bile, blood, liver and common hepatic bile duct tissue was collected. No evidence of biliary injury was observed following 30 minutes of warm ischaemia and six hours of reperfusion. However, severe small bile duct injury was present six hours after the administration of LPS, which was associated with impaired clearance of bile acids from 
the circulation. In this experiment we furthermore assessed the permeability of the blood-biliary-barrier (BBB) using horseradish peroxidase (HRP). Following intravenous injection, more HRP leaked across the BBB and appeared into bile in the LPS groups. These findings were associated with a significant decrease in Claudin-1 and claudin-3 gene expression, both genes encoding for important tight junction proteins. Furthermore, immunofluorescence staining for Zonnula occludens-1, showed markedly deranged tight junction morphology with a staining pattern not confined to the junctions of neighbouring cholangiocytes. The findings of this study highlighted the potency of endotoxins such as LPS to induce biliary injury.

In chapter 4 we aimed to determine the role of Kupffer cells in the development of LPS-mediated biliary injury. Kupffer cells become activated upon stimulation with LPS and we therefore hypothesised that in their absence, LPS mediated biliary injury would not occur. Rats were pre-treated with clodronate liposomes and exposed to sham operation, $1 \mathrm{mg} / \mathrm{kg}$ LPS administration or LPS administration combined with 30 minutes of warm hepatic ischaemia to $70 \%$ of the liver. Despite successful depletion of macrophages from the liver, no reduction in biliary injury was observed following six hours of reperfusion. In addition, increased BBB permeability persisted. As cholangiocytes themselves can play a role in innate immunity and respond to LPS in a toll-like receptor-4 dependant fashion, biliary concentrations of several cytokines and chemokines were assessed to gain insight into the local inflammatory milieu surrounding cholangiocytes. Monocyte chemoattractant protein-1 (Mcp-1) and tissue inhibitor of metalloproteinase-1 (Timp-1) were 8.3 and 10.3-fold increased following LPS administration or LPS combined with warm hepatic ischaemia. Macrophage depletion did not affect the levels of Timp-1 and Mcp-1. Results from this study show that macrophages do not play a pivotal role in the development of LPS-induced biliary injury. Instead, cholangiocytes themselves might be responsible for the induction of small bile duct injury however further studies are required to confirm this.

Results from the animal experiment prompted the study conducted in human organ donors as described in chapter 5. The concentration of endotoxins and other inflammatory mediators was assessed in portal blood from organ donors. We hypothesised that the concentration of inflammatory mediators was increased in portal blood collected from DCD donors due to a period of hypo-perfusion and hypoxia prior to organ retrieval. Based on the convincing results of the rat study we further hypothesised that these pro-inflammatory mediators such as endotoxins were responsible for the induction of biliary injury in DCD donors. In total, nine DCD donors and 21 brain death donors (DBD) were included in this study. No differences were found in the presence of pro-inflammatory mediators in portal blood between DCD and DBD donors, both by the direct measurement of endotoxin and by the use of a bioassay for inflammatory stimuli. Despite the absence of pro-inflammatory 
mediators, Kupffer cell activation was enhanced and prolonged in DCD donors. In addition, biliary injury could already be detected in DCD donors at the time of organ retrieval.

The second section of this thesis was aimed at the development of an ex vivo normothermic machine perfusion (NMP) protocol at our hospital. This system served as a platform to further study bile ducts of DCD donors and was also used as an assessment tool to determine graft viability of livers previously deemed unsuitable for transplantation. In Chapter 6 the different machine perfusion setups used in preclinical and clinical settings around the globe were reviewed. The aim of this systematic review was to determine the safety and applicability of machine perfusion. In total, 22 manuscripts were identified and all but one were published within the last five years. The corresponding author of each manuscript was approached to provide information regarding the safety of their perfusion protocol and none had encountered major technical complications that resulted in the loss of a liver graft. Both hypothermic perfusion as well as normothermic perfusion has been used prior to transplantation in a small case series and the results are promising. Equal or superior graft function is observed and hypothermic machine perfusion convincingly reduced the incidence of ITBS following transplantation of DCD grafts. However as NMP is the only modality that allows for graft assessment prior to transplantation, we proceeded to establish this technique at our centre.

An NMP protocol was developed at the Princess Alexandra Hospital and the first results were described in Chapter 7. Perfusate was pumped from the inferior vena cava using a centrifugal pump and flushed through an oxygenator with an inbuilt heat exchanger. Oxygen rich perfusate was then supplied directly to the hepatic artery whereas the portal vein was gravity fed via a reservoir. During the perfusion, amino acids, insulin and bile acids were continuously infused and a scavenger system was in place to recirculate leaked perfusate back into the closed system. To date, ten livers, all declined for transplantation, have been perfused using our custom-made perfusion circuit as described in chapter $\mathbf{8}$. Based on the currently available literature, lactate clearance within two hours of perfusion was used to distinguish viable from nonviable livers. Ultimately, seven livers were deemed viable whereas three livers were deemed non-viable. Compared to non-viable livers, viable grafts had significantly lower concentrations of potassium, AST and $y$-glutamyl transferase and increased urea concentrations in their perfusate. Furthermore at end of perfusion, liver morphology was preserved upon histological assessment.

As NMP was shown to have a protective effect on bile ducts in animal studies, we assessed the degree of injury to the common bile duct (CBD) at the time of organ donation, prior to the start of machine perfusion and at the end of perfusion in a 
semi-quantitative fashion. Unfortunately, severe biliary injury was observed in CBD sections collected at the end of perfusion, regardless of metabolic function. The degree of injury to the CBD however was not found to correlate with the degree of injury to the main hepatic ducts or the segmental ducts. These findings underline the need for a biomarker of biliary injury or viability in order to determine which grafts are unsuitable for transplantation due to a high risk of ITBS formation. This will be the focus of future studies.

Chapter 9 described the key findings of this thesis and discussed the results and implications for clinical practice. Furthermore, avenues for future research were described. 
Samenvatting 


\section{Samenvatting}

In Hoofdstuk 1 wordt in het een kort overzicht gegeven van de geschiedenis van de levertransplantatiechirurgie. Daarnaast worden de huidige klinische limitaties besproken en wordt het doel van dit proefschrift gepresenteerd.

Momenteel zijn er niet genoeg donorlevers beschikbaar voor alle patiënten op de wachtlijst waardoor er wordt overwogen om meer levers afkomstig van nonheartbeating donoren te gebruiken voor transplantatie. Echter is het gebruik van deze levers geassocieerd met de ontwikkeling van galwegstricturen. Dit heeft tot gevolg dat patiënten die een dergelijke lever ontvangen frequenter re-interventies ondergaan en uiteindelijk is re-transplantatie in eenderde van de gevallen noodzakelijk. De voornaamste doelstelling van dit proefschrift was om de pathofysiologie van galwegstricturen na transplantatie van een non-heartbeating donorlever in kaart te brengen. Om dit doel te bereiken werd er gebruik gemaakt van zowel een diermodel voor galwegschade als een ex-vivo normotherm machineperfusiesysteem voor humane levers.

In hoofdstuk 2 worden de resultaten van een review van alle Australische orgaandonoren tussen 2005 en 2014 gepresenteerd. Sinds de implementatie in 2008 is het aantal non-heart-beating orgaandonoren gestaag toegenomen. Echter werden levers afkomstig van deze donoren maar zelden gebruikt voor transplantatie, wat ertoe leidde dat de proportie niet geaccepteerde levers toenam van 42 (26\%) in 2005 naar 141 (43\%) in 2014. Een multivariabele regressieanalyse liet tevens zien dat nonheart-beating donatie de grootste onafhankelijke risicofactor was om een lever niet te accepteren voor transplantatie, gevolgd door hoge leeftijd, obesitas en diabetes. Naast het verkrijgen van een beter inzicht in de ontwikkeling van galwegstricturen kan ook het gebruik van machineperfusie worden overwogen om meer levers afkomstig van non-heart-beating donoren te gebruiken voor transplantatie. In de Australische staat Queensland werden 33 (41\%) non-heart-beating orgaandonoren geïdentificeerd die geschikte kandidaten zouden kunnen zijn voor machine perfusie.

In hoofdstuk 3 wordt de pathofysiologie van galwegschade verder bestudeerd. In de studie beschreven in dit hoofdstuk, werd gekeken naar de rol van lipopolysachariden (LPS) en ischemische-reperfusie op het ontstaan van galwegschade. Ratten werden blootgesteld aan $1 \mathrm{mg} / \mathrm{kg}$ LPS, warme ischemie van de lever voor 30 minuten of een combinatie van beiden. Eén of zes uur na de ingreep werden vervolgens bloed, gal, lever en galwegweefsel verzameld. Leverischemie gedurende 30 minuten en reperfusie gedurende zes uur bleek geen nadelig effect te hebben op de galwegen. Echter er ontwikkelde zich wel ernstige galwegschade zes uur na blootstelling aan LPS of ischemie gecombineerd met LPS, welke gepaard ging met verminderde hepatische 
klaring van galzouten uit de systemische circulatie. In dit experiment werd horseradish peroxidase (HRP) gebruikt om de permeabiliteit van de bloed-gal-barrière te testen. Deze barrière bestaat uit naburige cholangiocyten die met elkaar verbonden zijn middels tight-junctions. Na intraveneuze injectie werd meer HRP in gal gevonden in de groepen ratten die behandeld waren met LPS, dit duidt op verminderde functie van de bloed-gal-barrière. Deze bevinden waren geassocieerd met een verlaagde genexpressie van claudin-1 en claudin-3, die beiden coderen voor tight-junctioneiwitten. Tevens liet een immunufluoresente kleuring een sterk verstoord kleuringspatroon zien van het tight-junctioneitwit zonula-occludens-1. Resultaten van deze rattenstudie tonen aan dat endotoxines zoals LPS zeer potent zijn in het veroorzaken van galwegschade.

In hoofdstuk 4 wordt de rol van Kupffercellen in de ontwikkeling van LPS gemedieerde galwegschade bestudeerd. Bloodstelling aan LPS kan leiden tot activatie van Kupffercellen. De hypothese was dat LPS-gemedieerde galwegschade niet zou optreden in afwezigheid van Kupffercellen. Ratten werden voorbehandeld met clodronaatbevattende liposomen waarna ze werden blootgesteld aan LPS of een combinatie van LPS en warme ischemie van de lever. Ondanks het feit dat Kupffercellen succesvol werden verwijderd uit de lever, ontstond nog steeds ernstige galwegschade. Tevens bleef de functie van de bloed-gal-barrière verminderd. Om een beter inzicht te krijgen in het inflammatoire milieu rondom cholangiocyten, werd de concentratie van verschillende cytokines in gal gemeten. De hoeveelheid monoctytechemoattractant protein-1 (Mcp-1) en tissue inhibitor of metalloproteinase-1 (Timp-1) waren $8.3 x$ en $10.3 x$ verhoogd na blootstelling aan LPS en LPS gecombineerd met warme ischemie. Kupfferceldepletie had geen effect op de concentratie Timp-1 en Mcp-1. Op basis van deze studie kan worden geconcludeerd dat Kupffercellen geen prominente rol spelen in de ontwikkeling van LPS-gemedieerde galwegschade. Echter lijken cholangiocyten zelf mogelijk een rol te spelen.

De bevindingen in de rattenstudies leidden tot de initiatie van een studie met humane orgaandonoren welke wordt beschreven in hoofdstuk 5. De concentratie van endotoxines en andere pro-inflammatoire factoren werden bepaald in portaal bloed van orgaandonoren. De hypothese was dat portoveneus bloed van non-heart-beating donoren een hogere concentratie pro-inflammatoire factoren zou bevatten als gevolg van hypoxie en hypoperfusie van de darm, gedurende orgaandonatie. Tevens werd verondersteld dat pro-inflammatoire factoren zoals endotoxines verantwoordelijk waren voor de inductie van galwegschade in non-heart-beating donorlevers. In totaal werden negen non-heart-beating en 21 heart-beating orgaandonoren geïncludeerd in de studie. Er werd geen verschil gevonden in de hoeveelheid pro-inflammatoire factoren tussen deze twee typen orgaandonoren. Desondanks, werd er een verhoogde activatie van macrofagen in de lever vastgesteld; en galwegbiopten van 
non-heart-beating donoren vertoonden meer galwegschade in vergelijking met die van heart-beating donoren.

Het tweede gedeelte van dit proefschrift is met name gericht op het gebruik van normotherme machine perfusie (NMP) van humane marginale levers. Dit systeem werd niet alleen gebruikt om te differentiëren tussen levers die geschikt zijn voor transplantatie en levers die uiteindelijk onvoldoende functioneren, maar ook om de galwegen van non-heart-beating donoren verder te bestuderen. Allereerst werd systematisch de beschikbare literatuur bestudeerd in hoofdstuk 6 met als doel de veiligheid en bruikbaarheid van machine perfusie protocollen in pre-klinisch en klinisch onderzoek in kaart te brengen. In totaal werden 22 publicaties geïdentificeerd die hypotherme, midtherme of normotherme machineperfusie-protocollen beschreven welke elke werden gezien als veilig en bruikbaar. Een klein aantal hypotherm en normotherm geperfuseerde marginale levers zijn inmiddels gebruikt voor transplantatie met goede resultaten. Tevens ontwikkelen patiënten die hypotherm geperfuseerde non-heart-beating donorlevers ontvingen significant minder galwegstricturen in vergelijking met patiënten die een niet geperfuseerde non-heartbeating donorlever ontvingen. Echter aangezien NMP als enige methode de mogelijkheid biedt om leverfunctie te testen alvorens de lever wordt getransplanteerd, werd besloten om een protocol op te zetten in ons centrum in Brisbane (Australië).

Er werd een NMP protocol ontwikkeld in het Princess Alexandra Ziekenhuis, in Brisbane (Australië) en de eerste resultaten worden besproken in hoofdstuk 7. Het perfusiesysteem werkt als volgt: perfusievloeistof (bloed van de orgaandonor) werd uit de vena cava inferior gepompt met behulp van een centrifugaal pomp, waarna de perfusievloeistof door een oxygenator stroomde welke tevens de perfusievloeistof opwarmde tot 37 graden Celcius. Zuurstofrijk bloed werd vervolgens direct naar de a. hepatica geleid terwijl de vena porta werd gevoed door middel van een reservoir. Tijdens de perfusie werden constant aminozuren, insuline en galzouten toegediend en werd er elke vier uur een bolus heparine gegeven om embolieën te voorkomen. Over de afgelopen twee en een half jaar werden tien levers, voorheen bestempeld als ongeschikt voor transplantatie, middels dit systeem geperfuseerd (hoofdstuk 8). Lactaatklaring gedurende de eerste twee uur op de perfusiemachine werd gebruikt om te differentiëren tussen levers die geschikt zijn voor transplantatie en levers die uiteindelijk onvoldoende functioneren. Op basis van dit criterium zouden zeven normotherm geperfuseerde levers mogelijk bruikbaar zijn geweest voor transplantatie. Vergeleken met levers die onbruikbaar werden geacht hadden bruikbare levers een lagere concentratie kalium, AST en $\gamma$-glytamyl transferase en een hogere concentratie ureum in de perfusievloeistof. Aan het einde van de 
perfusieperiode van gemiddeld zes uur werden geen histologische tekenen van leverschade gevonden.

Eerdere dierstudies hebben aangetoond dat NMP schade aan de galwegen kan verminderen. Om de invloed van NMP op humane galwegen te beoordelen werden er biopten genomen van de ductus choledochus tijdens orgaandonatie en aan het begin en eind van NMP. Helaas was schade aan de ductus choledochus onvermijdelijk, ongeacht de metabole functie van de lever. Echter, de hoeveelheid schade aan de ductus bleek niet te correleren aan de hoeveelheid schade aan de grote intrahepatische galwegen en de segmentale galwegen. Toekomstige studies dienen zich daarom te focussen op het ontwikkelen van een biomarker voor galwegschade, om zo veilig non-heartbeating donorlevers te kunnen gebruiken voor transplantatie.

In hoofdstuk 9 worden de belangrijkste bevinden van dit proefschrift beschreven en worden de implicaties voor de kliniek bediscussieerd. Tevens wordt de focus van toekomstige studies besproken. 
Valorisation 


\section{Valorisation}

This chapter will discuss how the research results presented in this thesis could be used in clinical practice. The implications of the study results to the wider community will be described. Lastly, the necessary steps to implement machine perfusion into clinical practice will be reviewed.

\section{Implications of research results for the wider community}

In Australia, there has been a steady increase in the number donor livers deemed unsuitable for transplantation over the last ten years. Despite the strong wishes of donor families to use the organs of their loved ones to save the lives of others, the number of liver declined for transplantation increased from $26 \%$ in 2005 to $43 \%$ of in 2014. On the other hand, annually about $10 \%$ of patients with end stage liver disease waiting to undergo life-saving liver transplantation became ineligible as their disease progressed beyond acceptance criteria for transplantation. The decreasing rate of liver utilisation is not only a pressing problem in Australia, but has been reported around the globe. In the United Kingdom, the proportion of livers used for transplantation has dropped by $16 \%$ between $2005-06$ and $2014-15$. $^{1,2}$ Furthermore, Orman et al. found that in the US, liver non-use increased from $15 \%$ in 2004 to $21 \%$ in $2010,{ }^{3}$ which was accompanied by declining annual liver transplant rates.

If we could make better use of the organs currently available, it would mean that the wishes of more donor families could be honoured. Furthermore, as this could be achieved without an increase in the number of organ donors, it would be the most cost effective way to save the lives of patients that succumb to their disease while waiting to undergo liver transplantation.

Currently a large proportion of livers declined for transplantation are from organ donors who donate their organ after the cessation of circulation (donation after cardiac death, DCD). The use of these liver grafts is currently limited by the slightly increased risk of primary non-function, especially when transplanted into a high-risk recipient. More importantly, the development of ischaemic type biliary strictures (ITBS) in up to one in three recipients limits long-term graft survival. ${ }^{4,5}$ As a results, the cost per life year gained is far greater in DCD donors where $€ 112,376$ was spent per annum compared to $€ 88,913$ per year for those that receive a liver donated after brain death. ${ }^{6}$

The aim of this thesis was to characterise the sequence of events associated with the development of ITBS, as this is currently the most limiting factor in the utilisation of DCD livers. Improved knowledge about the pathophysiology of ITBS may help find new therapies or preventing measures, and these may benefit the wider community. In 
context, the use of normothermic machine perfusion was explored as a tool to assess graft function of livers currently declined for transplantation Combined, these two avenues of research have an impact on the wider community as a whole as liver disease and organ donation touches the lives of many patients worldwide.

\section{The implementation of normothermic machine perfusion}

The application of normothermic machine perfusion (NMP) has several advantages over the use of conventional simple cold storage. First of all, the quality of marginal donor organs can be preserved which would allow for the use of more livers currently declined for transplantation. ${ }^{7}$ Furthermore, it possibly allows for extended periods of preservation, which in turn facilitates long distant transport but also improves transplant logistics as the transplant can take place during daytime hours. ${ }^{8}$ In addition, results from several animal studies have indicated that NMP is preventing biliary injury, which could reduce the incidence of ITBS. ${ }^{9,10}$ Equally importantly, graft function can be assessed during perfusion at $37^{\circ} \mathrm{C}$, which could avoid transplanting a nonviable graft.

An NMP protocol was established at the Princess Alexandra Hospital and ten livers declined for transplantation were successfully perfused. Based on the viability criteria previously described by other centres, ${ }^{7,11,12}$ seven could potentially have been used for transplantation. Over the last 2 years, 146 DCD liver grafts were declined for transplantation in Australia. If $70 \%$ of these grafts could have been recovered using NMP, this would have increased the donor pool by $16 \%$. A similar prediction of the effect of NMP on organ donor numbers was made by Mergental et al.. ${ }^{13}$ Based on the number of livers declined for transplantation and the results of their pilot study, they estimated that a $15 \%$ increase in the total number of donor livers could be achieved in the United Kingdom.

Based on the experience with NMP described in this thesis and reports of other centres that have implemented the technique in clinical practice, the next aim is to start using those livers that are deemed viable during NMP for transplantation.

\section{What is required to implement NMP in clinical practice?}

\section{Machine perfusion post static cold storage or in transit}

Currently, NMP has been applied following a period of cold storage as well as during transit. ${ }^{8,12}$ Although in transit perfusion limits the period of cold preservation injury to about one hour, it comes with some logistical challenges. It would mean that a new 
vehicle fitted with a main power supply needs to be purchased to facilitate the transport of this large piece of equipment. However air-transportation is often required in countries such as the United States, Canada and Australia and the use of NMP in a small airplane has not previously been performed. Furthermore, banked matched blood will have to be taken to the donor hospital to serve as perfusate during NMP although the blood may not be used if a DCD donor does not die within the currently accepted time frame. Lastly, in some instances the airplane is shared between the abdominal and cardiothoracic team when retrieving organs from donors outside the metropolitan area. As the maximum cold ischaemic time of a heart is already in jeopardy when flight time exceeds 2 hours, there may be time restrictions in establishing NMP for the donor liver prior to transportation.

Therefore in Australia, NMP will probably only be initiated at the recipient hospital following a period of conventional cold storage.

\section{Organ donor inclusion criteria}

An important consideration is which liver grafts require NMP prior to transplantation. Some reported studies have included "within criteria" grafts in their perfusion studies (8). These livers are however already acceptable for transplantation using current empirical criteria and the application of NMP exposes them to the potentially catastrophic effects of pump failure. Furthermore it will not increase the number of grafts available for transplantation but it may increase the costs associated with using them. In fact, as the appropriate criteria to determine viability are still being established, perfusion of "standard criteria" donor livers could potentially lead to a reduction in the number of transplants. One group of patients that could benefit from machine perfusion of "standard criteria" grafts are those with a high model for endstage liver disease (MELD) in the intensive care. Machine perfusion reduces the reperfusion injury and this could reduce the incidence of post-reperfusion syndrome during transplantation. ${ }^{14}$

However as increasing the number of donor livers available for transplantation is the primary goal, livers currently declined for transplantation should be the target of NMP. Furthermore, the use of livers with evidence of structural damage or organ donors infected with hepatitis B, C or HIV should be avoided. Initially, warm ischaemic time of DCD donors will be limited to 30 minutes, which can be extended once we become more experienced.

\section{Criteria for viability assessment}

A standardised protocol will be established outlining the criteria used to determine viability. Based on the pre-clinical results described in this thesis, lactate clearance below $2 \mathrm{mmol} / \mathrm{L}$ within two hours will be the main component. Furthermore, the liver 
needs to have a satisfactory macroscopic appearance and both the surgeons as well as the anaesthetist involved in the care of the recipient need to agree on proceeding. While the recipient is prepared for transplantation, machine perfusion will continue until transplantation. This period is anticipated to be up to six hours.

\section{Recipient selection}

The selection of suitable recipients of these high-risk NMP livers is crucial in obtaining favourable long-term outcomes. In countries such as Australia and the United Kingdom, no nationwide liver transplant waiting list exists and organ allocation is performed by individual transplant centres. Patients with hepatocellular carcinoma could be suitable low-risk recipients to receive an NMP perfused liver. These patients often do not have end stage liver disease with portal hypertension and ascites, which makes the transplant operation more straightforward from a technical perspective.

\section{Commercial machine versus custom-made setup}

Currently, two commercial machines have been used for normothermic liver perfusion in clinical studies in Europe and the United Kingdom however both are not approved by the Therapeutic Goods Administration for use in Australia. ${ }^{8,15}$ These machines cost up to AUD 260,000, which excludes the disposables (personal communication). These are priced between AUD 9000 and AUD 18,000 per perfusion. The perfusion setup described in this thesis, is comprised of components used for ECMO, and costs AUD 1500 per perfusion.

As experience was gained with our custom made setup and it is significantly cheaper than the commercially available machines, we will continue to use the perfusion machine described in this thesis.

\section{Cost implications for transplant program}

An increase in donor numbers will come at a price. Not only is machine perfusion more expensive than conventional static cold storage, staff and facility costs needed to run them have to be considered. Currently, the kidneys from DCD donors are often the only abdominal organs retrieved from DCD donors and their retrieval is performed by an urologist or general surgeon. If the livers of those donors are considered for transplantation, this will mean that HPB surgeons will have to perform more retrievals and an increase in surgical staff will need to be considered. Despite the increase in cost in the short term, it will reduce the cost required for ongoing care of patients with end stage liver disease. Furthermore, if the incidence of biliary complications can be limited by the use of NMP, it will significantly reduce the costs of ongoing care for liver transplant recipients. 
In conclusion, machine perfusion will have a distinct impact on global transplantation practices in the near future as it will ultimately lead to a significant increase in the number of livers available for transplantation. 


\section{References}

1. NHS Blood and Transplant. Organ Donation and Transplantation Activity Report 2014/15. 2014-2015.

2. Transplant NBa. Organ Donation and Transplantation Activity Report 2005/06. 2005/06.

3. Orman ES, Barritt ASt, Wheeler SB, Hayashi PH. Declining liver utilization for transplantation in the United States and the impact of donation after cardiac death. Liver Transpl. 2013;19(1):59-68.

4. Blok JJ, Detry O, Putter H, Rogiers X, Porte RJ, van Hoek B, et al. Longterm results of liver transplantation from donation after circulatory death. Liver Transpl. 2016;22(8):1107-14.

5. Dubbeld J, Hoekstra H, Farid W, Ringers J, Porte RJ, Metselaar HJ, et al. Similar liver transplantation survival with selected cardiac death donors and brain death donors. Br J Surg. 2010;97(5):744-53.

6. van der Hilst CS, Ijtsma AJ, Bottema JT, van Hoek B, Dubbeld J, Metselaar HJ, et al. The price of donation after cardiac death in liver transplantation: a prospective cost-effectiveness study. Transpl Int. 2013;26(4):411-8.

7. Karangwa SA, Dutkowski P, Fontes P, Friend PJ, Guarrera JV, Markmann JF, et al. Machine Perfusion of Donor Livers for Transplantation: A Proposal for Standardized Nomenclature and Reporting Guidelines. 2016.

8. Ravikumar R, Jassem W, Mergental H, Heaton N, Mirza D, Perera MT, et al. Liver transplantation after ex vivo normothermic machine preservation: a Phase 1 (first-in-man) clinical trial. Am J Transplant. 2016.

9. Liu Q, Nassar A, Farias K, Buccini L, Baldwin W, Mangino M, et al. Sanguineous normothermic machine perfusion improves hemodynamics and biliary epithelial regeneration in donation after cardiac death porcine livers. Liver Transpl. 2014;20(8):987-99.

10. Op den Dries S, Karimian N, Westerkamp AC, Sutton ME, Kuipers M, Wiersema-Buist J, et al. Normothermic machine perfusion reduces bile duct injury and improves biliary epithelial function in rat donor livers. Liver Transpl. 2016;22(7):994-1005.

11. Watson CJ, Kosmoliaptsis V, Randle LV, Russell NK, Griffiths WJ, Davies S, et al. Preimplant Normothermic Liver Perfusion of a Suboptimal Liver Donated After Circulatory Death. Am J Transplant. 2016;16(1):353-7.

12. Perera T, Mergental H, Stephenson B, Roll GR, Cilliers H, Liang R, et al. First human liver transplantation using a marginal allograft resuscitated by normothermic machine perfusion. Liver Transpl. 2016;22(1):120-4.

13. Mergental H, Perera MT, Laing RW, Muiesan P, Isaac JR, Smith A, et al. Transplantation of Declined Liver Allografts Following Normothermic Ex-Situ Evaluation. Am J Transplant. 2016.

14. Quillin RC, 3rd, Guarrera JV. In 10 Years Machine Perfusion for Liver Preservation Will Be Universal. Liver Transpl. 2016.

15. Sutton ME, Op Den Dries S, Karimian N, De Boer MT, Wiersema-Buist J, Gouw ASH, et al. Criteria for viability assessment of discarded human donor livers during ex-vivo normothermic machine perfusion. Liver Transplant. 2014;20:S240. 
Acknowledgements 


\section{Acknowledgements}

\section{Professor Jonathan Fawcett}

Jonathan, I cannot thank you enough for all your support throughout this journey. Ever since I first started as a medical student with the transplant department you have helped making this PhD a reality. From all those Saturday morning Skype sessions when I was still in Maastricht to lunch meeting at café Essence across the road, you were always available to discuss results and future directions. Furthermore, establishing the machine perfusion project would never have been possible without your input and the sacrifice of your precious spare time. You always knew how to push my work to the next level and I am forever grateful for your contribution. You have made me feel at home in this lovely country and you always made sure I was managing well. Thank you from the bottom of my heart; you are someone I have immense respect for and truly look up to.

\section{Professor Kees Dejong}

Dear Kees, I can still remember the day I walked in to your office hoping you would support my wild idea of starting a Joint PhD with the University of Queensland, Australia. You asked me where I wanted to go and when I said that I was going to Brisbane you mentioned that Professor Strong, the founder of the transplant unit in Brisbane, once saved your Whipple operation when you were a young surgical fellow in Edinburgh. I guess it is a small world we live in! Thank you so much for your continuous support and intellectual input, especially during my time in Maastricht at the end of 2015. You always knew how to keep me on track, and prevent me from taking on even more projects. Your editorial skills have never ceased to amaze me; you have always been the one picking up on my silly English mistakes, even in my emails! Although my plan is to stay in Australia for a little while longer, I'm certainly looking forward to working with you again in the future.

\section{Dr Kim Bridle}

Kim, I do not even know where to start! All those long hours we spent in the animal house together. All day in a room without windows, no cell phone reception, isolated from the rest of the world. I guess the best example of how disconnected we were from the outside world is when I sent you a message about how annoyed I was that there had been a power outage at the animal facility and I lost an entire day's work. Little did I know that the reason for the power outage was a massive storm, which had broken almost every single window in your house! Kim you have been a tremendous support ever since you collected me from Jonathan's office at the PA three and a half years ago. You taught me how to write an ethics application, how to plan and conduct experiments and how to troubleshoot when things were not exactly going as planned. 
Thank you so much for all your effort and support, without you this PhD would not have been possible!

\section{Professor Darrell Crawford}

Darrell, when Jonathan suggested that I come and work in your lab, I could not have anticipated how great it would be. I had never worked in a laboratory before but I was up for a challenge. And boy a challenge it was! Thank you for all the fruitful discussions, although you must have found my Dutch directness quite irritating at times. Your clinical knowledge allowed my results to be given perspective and you were there for me in my ongoing negotiations with the graduate school. In recent years I have heard numerous stories of the good old times when you still had time to attend conferences. I'm looking forward to enjoying these with you in the future.

Laurence, Nish, Lesley, Bijay, Sean, Aparna and Jason

Thank you for making the last three and a half years such an amazing experience! Without your help I would never have been able to succeed. Thank you Lesley for teaching me how to hold a pipette and all the other technical parts of lab work. I have to say performing a western blot will never be my favourite but thanks to you I now know how to perform one. Nishie, thanks to you I now have about a dozen nicknames to choose from. And if you are ever bored, your Dutch language skills are phenomenal; you should do something with that! Dr. Laurence Britton, your knowledge as a hepatologist has been of great value and your input has definitely improved the quality of my work, thank you.

Although I have never met any of you, I would like to thank the donor families from the bottom of my heart. During the hardest time in your life you made the generous decision to donate the organs of your loved ones to give someone else a second chance at life. Without your courageous decision I would not have been able to conduct my research. Thanks to you, more livers will become available for transplantation for those who desperately need them.

Dr David Lockwood, Dr Peter Hodgkinson, Dr Anthony Griffin, Dr Thomas O'Rourke, Dr Shin Yeung and Dr Kellee Slater

Dear David, Peter, Tony, Tom, Shin and Kellee, thank you ever so much for your patience and contributions to my research projects. You were always willing to help with the collection of samples and I have gained a tremendous amount of knowledge about surgical anatomy. I have a great deal of respect for the work you do and your knowledge and enthusiasm will form an inspiration for the rest of my career. 
Tina, Angela, Fran, Bianca, Cheryl, Niamh, Britt, Jade, Megan, Angela, Shaun, and all other members of DonateLife Queensland, thank you so much for making me feel a part of the team! Some of you I have known for more than five years, and you have always looked after me during organ retrievals. I'm forever grateful for your patience and ongoing support over the last few years.

Marie, Lisa, Julie and Sue, although we mostly spoke over the phone in the early hours of the morning, I would like to thank all of you for your help achieving my goals. Glenda, thanks for your help with the liver utilisation project. Without your input I would have spent hours and hours going through every single individual donor file in the archives. Geraldine, your help with my first study in transplant recipients was greatly appreciated. I had no idea how to go about it or who to talk to, so without your knowledge it would have been a disaster!

\section{Dr Andrew Simpson}

Dear Andrew, your help with setting up the machine perfusion study was of incredible value. Without you we would never have been able to perfuse a single liver. I am very grateful for all of your assistance, your willingness to participate in a research project and devoting your precious spare time.

\section{Dr Elizabeth Forrest}

Dear Liz, I would like to thank you for all your help during several liver perfusions. You were always happy to travel, even at short notice, and your presence definitely made things much easier for me. The speed by which you can collect data from a database is remarkable and I am very excited to see that you may have been infected with the "research virus". I am sure you will go on to achieve great things!

\section{Dr Catherine Campbell}

Cate, I can remember the first time I came to your office at Butterfield Street. I was so excited about the rat ischaemia project but unfortunately there appeared to be no histological signs of biliary injury. I was so disappointed when I found out. Luckily I persisted and your help and input has been of great value. You must have spent hours sitting behind the microscope scoring the hundreds of human and rat bile duct sections for me, for which I am forever grateful. I would like to thank you and the rest of Envoi Pathology for all your assistance with my projects, specifically the machine perfusion study.

Associate Professor Frank Schaap and Professor Steven olde Damink

Frank and Steven, your knowledge and contributions have lifted my work to a whole new level! It was great spending time working in your labs and being part of an 
amazing group of young ambitious doctor-scientists. It was definitely too short but it nevertheless has shaped the work presented in this thesis, thank you.

Dear Frans, Jaqueline, Audrey, Kim, and all the others that are part of the PhD crew at the department of surgery in Maastricht, although it was only short I had an terrific time at UM. Frans, roomie, we used to share the director's office, it's a shame we eventually had to move to "where the others work". Guys, I had a great time, and you are all more than welcome to come and visit me in Australia!

A big thank you to all the people at the Gallipoli medical Research Foundation. You have made my time at Greenslopes enjoyable! The work that is being conducted at the labs but also in the clinical trials unit is ground breaking and definitely contributes to a better understanding and treatment for a wide range of diseases. Keep up the good work!

I would also like to thank the PA research foundation, the University of Queensland as well as the Gallipoli Medical Research Foundation for their funding and support which allowed me to conduct my PhD. I would like to thank the Gastroenterological Society of Australia and the Transplant Society of Australia and New Zealand for their generous travel support. I am honoured to be a recipient of the University of Queensland Graduate School International Travel Award, which allowed me to attend the 2015 annual Liver Meeting from the American Association for the Study of Liver Diseases in Boston.

Dear Elena and Katrin, my roomies, without you guys I might not have started a PhD in Australia! It all started in Westella and the both of you showed me how great the Australian way of life is. When I returned, it was such a good feeling moving back to West End and living with the both of you. I could not have been luckier and I thank you both for all the wonderful times, the great trips, the lovely dinners and the endless amounts of fun!

Dear Julie, Emma, Fabien, Selene, Ann-Katrin, Claudio, Adrian, Victoria, Javiera, Arzu, Marie Laure and Bonnie, my Australian international family, where would I have been without all of you! Thank you guys for the time we spent together. Camping on Moreton, our trip to Girraween, Australia day in Coolum, numerous trips to Straddie, up the Sunshine Coast, down the Gold Coast, Sunshine coast hinterland, I have had the most amazing times with all of you. I guess by now I know that when I am in charge of shopping for 15 people, I should NOT forget to buy lots of chocolate! Although most of you have left Brissie and moved on to do great things around the globe, I will never forget how we spent every Friday night having dinner and drinks in West End and every Saturday morning under the tree near the gypsy coffee cart at the 
markets. All of you have a special place in my heart and I hope I now have a place to stay when I visit San Diego, Oxford, Osnabruck, Marseille, Paris, Boston, Guanajuato, San Salvador de Jujuy and Santiago!

Dear Mechteld and Simone, my buddies, a.k.a the three best friends that anyone can have! Without you guys this PhD would never have been a success. I am missing the good old times when Mechteld would come over for dinner at least twice a week, and we would go out to Café Sjiek on Fridays. The trips we have made together to Prague, Perth and Japan have been memorable! And even though I have been away on the other side of the world, you always kept me informed of Mestreech's gossip! I can't wait for the both of you to come over to Brisbane to visit me!

Dear mum and Dad, remember that time in 2011 when I told you guys that I was thinking of doing my PhD in Australia? The both of you had just travelled half way around the world to come and see me, you must have thought (or wished) that I was joking! It has been very hard at times to be this far away from you, the people that taught me everything. Mum and dad, you have always supported my decisions $100 \%$ and I am forever grateful. Loes, Thijs, Opa en Oma, and the rest of the family, thank you so much for all the support you provided throughout this journey. You might be far away but those postcards, letters, text messages, Skype calls and Facetime sessions definitely made things a lot easier!

Dear Rich, I don't know how you managed to put up with me for the last 2.5 years! All the trips and plans that had to be cancelled because I had to go and help out on a transplant. Or the times you had to pick me up from the hospital because I was too tired to drive (or had a broken finger). You were always there to help me, no questions asked. I furthermore have a great deal of respect for the way you combine a full-time job with studying while still being there to support me. It has been very hard at times but you have always managed to succeed. I am looking forward to finally show you the beautiful country I grew up in and introduce you to all my friends and family in the Netherlands. Rich, you make me feel like the luckiest girl in the world! 
List of publications 


\section{List of publications}

\section{Papers}

J Reiling, KR Bridle, M Gijbels, FG Schaap, L Jaskowski, N Santrampurwala, L Britton, CM Campbell, SWM Olde Damink, DHG Crawford, CHC Dejong, J Fawcett, Low-dose lipopolysaccharide causes biliary injury by blood biliary barrier impairment in a rat hepatic ischemia/reperfusion model. Liver Transplantation, 2016 Nov 23. doi: 10.1002/It.24681

L Britton, L Jaskowski, K Bridle, N Santrampurwala, J Reiling, N Musgrave, V.N Subramaniam, D Crawford, Heterozygous Hfe gene deletion leads to impaired glucose homeostasis but not liver injury in mice fed a high calorie diet. Physiological Reports, 4 (12), 2016, e12837, doi: 10.14814/phy2.12837

J Reiling, DSR Lockwood, AH Simpson, CM Campbell, KR Bridle, N Santrampurwala, LJ Britton, DHG Crawford, CHC Dejong, J Fawcett, Urea production during Normothermic Machine Perfusion- Price of Success? Liver Transplantation, Liver Transplantation, 2015 May;21(5):700-3

J Reiling, D Johnson, P Kruger, P Pillans and D Wall, Association of pre-transplant statin use with delayed graft function in kidney transplant recipients, BMC Nephrology 2012, 13:111

\section{Conference abstracts}

J Reiling, A Simpson, KR Bridle, D Lockwood, N Santrampurwala, L Britton, CHC Dejong, DHG Crawford, J Fawcett, The application of normothermic machine perfusion to bench test human donor livers. Journal of Gastroenterology and Hepatology (2016) 31 (suppl. 2)

J Reiling, KR Bridle, N Santrampurwala, LJ Britton, C Campbell, DHG Crawford, CHC Dejong, J Fawcett, Lipopolysaccharides increase bile toxicity and induce severe biliary injury in an LPS enhanced ischaemia-reperfusion model. 2016, HPB;18:e126-e127.

J Reiling, KR Bridle, C Campbell, N Santrampurwala, L Britton, CHC Dejong, A Cohen, DHG Crawford, J Fawcett, Pre-treatment with TIMP-3 prevents the development of biliary injury in an LPS enhanced ischaemia-reperfusion animal model. Journal of Gastroenterology and Hepatology (2015) 30 (suppl. 3) 
N Santrampurwala, KR Bridle, J Reiling, LJ Britton, LA Jaskowski, VN Subramaniam, DHG Crawford. Free fatty acid treatment reduces BMP6 signalling in AML12 hepatocytes. Journal of Gastroenterology and Hepatology (2015) 30 (suppl. 3)

L Britton, K Bridle, J Reiling, N Santrampurwala, L Wockner, H Ching, V Subramaniam, D Crawford, L Adams. Serum adipokines in response to venesection in patients with non-alcoholic fatty liver disease. Journal of Gastroenterology and Hepatology (2015) 30 (suppl. 3)

J Reiling, KR Bridle, C Campbell, N Santrampurwala, L Britton, CHC Dejong, A Cohen, DHG Crawford, J Fawcett, Pre-treatment with TIMP-3 prevents the development of biliary injury in an LPS enhanced ischaemia-reperfusion animal model. Hepatology (2015) 62 (suppl. 1)

N Santrampurwala, KR Bridle, J Reiling, LJ Britton, LA Jaskowski, VN Subramaniam, DHG Crawford. Free fatty acid treatment reduces BMP6 signalling in AML12 hepatocytes. Hepatology (2015) 62 (suppl. 1)

J Reiling, KR Bridle, N Santrampurwala, L Britton, DHG Crawford, CHC Dejong, J Fawcett, The potential role of lipopolysaccharides in the development of biliary injury in an animal partial liver ischaemia model. Journal of Gastroenterology and Hepatology (2014) 29 (suppl.2)

J Reiling, KR Bridle, N Santrampurwala, U Britton, DHG Crawford, CHC Dejong, J Fawcett, Successful ex-vivo normothermic oxygenated machine perfusion of human donor livers. Hepatology (2014) 60 (suppl. S1)

J Reiling, KR Bridle, N Santrampurwala, UJ Britton, DHG Crawford, CHC Dejong, J Fawcett, Successful ex vivo normothermic oxygenated machine perfusion of human donor livers. Journal of Gastroenterology and Hepatology (2014) 29 (suppl.2) 
Curriculum vitae 


\section{Curriculum vitae}

Janske Reiling was born on October 31, 1987 in Maarheeze, the Netherlands. After obtaining her Atheneum degree at the Strabrecht College in Geldrop in 2005, she went on to study medicine at the University of Maastricht. During her fourth year she went to South Africa for her ophthalmology rotation and this sparked her passion for travel. In the following year she travelled to Brisbane, Australia, for her elective rotation as well as a research participation. Here she was introduced to the liver and kidney transplant department of the Princess Alexandra Hospital, a place that would play a vital role in her future career. She completed her research project on the role of statins in the onset of graft dysfunction following kidney transplantation under the supervision of Professor Darrell, professor David Johnson and associate Professor Peter Kruger. As the combination of living in Australia and a PhD in the transplant field seemed appealing, she decided to eventually come back to Brisbane.

Upon her return to the Netherlands she completed her participation in Healthcare at the Atrium Medical Centre in Heerlen under the supervision of dr W.G. van Gemert and obtained her medical degree in December 2011. During the following year she worked as a surgical resident at Maastricht University Medical Centre, The Netherlands, while finalising the application process to start a PhD in Brisbane. In April 2013 the necessary paperwork was finalised and she started her PhD under the joint supervision of Professor Jonathan Fawcett and Professor Kees Dejong at the University of Queensland and the University of Maastricht. In October 2016 she will commence her medical career in Australia at Greenslopes Private Hospital in Brisbane. 


\section{Appendix}

University of Queensland thesis preliminary pages 


\title{
Appendix
}

\begin{abstract}
Liver transplantation is the preferred treatment option for end stage liver disease. The demand for donor livers greatly exceeds the supply and organs from more marginal donors are being considered for transplantation. An important group of such donor livers are those obtained by donation after circulatory death (DCD). These livers are more susceptible to cold preservation injury and their use has been associated with a slight increased incidence of primary non-function in Australia. More importantly, biliary complications such as ischaemic type biliary strictures (ITBS) are more prevalent in recipients receiving a DCD liver resulting in a higher rate of reintervention, re-transplantation and inferior long-term graft survival. This thesis describes a series of studies examining potential factors responsible for the development of biliary injury. Furthermore, a normothermic hepatic machine perfusion protocol was established at our centre and used as a platform to study the development of biliary injury in human livers.
\end{abstract}

A review of all adult Australian organ donors between 2005 and 2014 revealed that there was a $17 \%$ increase in the number of livers deemed unsuitable for transplantation, mainly attributable to the rise in DCD donors. Over the study period, only 62 (13\%) livers from DCD donors were used for transplantation. In addition, advanced donor age, obesity as well as diabetes were associated with liver non-use.

The pathophysiology of biliary stricture formation following transplantation remains largely unknown and better insights into the sequence of events leading to their development are urgently needed. The work in this thesis has shown that endotoxins, in the form of lipopolysaccharides (LPS), are potent inducers of small but not large bile duct injury. Following six hours of administration of $1 \mathrm{mg} / \mathrm{kg}$ LPS, small bile ducts were severely affected with biliary epithelial cells showing significant degenerative changes, infiltration of inflammatory cells and ductular proliferation. This was further associated with an increased permeability of the blood-biliary-barrier and the development of clinical features of cholestasis.

Macrophages are activated by LPS and to determine their role in LPS-induced biliary injury, an additional group of animals was pre-treated with clodronate liposomes prior to LPS exposure. These macrophage depleted animals showed a similar degree of biliary injury in response to LPS, which suggests that macrophages don't play a crucial role in the pathophysiology of LPS-induced injury. 
These findings prompted the initiation of a prospective patient cohort study aimed to examine the levels of endotoxins and other pro-inflammatory mediators during DCD organ donation. Portal blood samples were collected from DCD donors and the inflammatory propensity of these samples was compared to samples collected from donors who donated after brain death (DBD). Using a cell-based assay as well as a Limulus Amebocyte Lysate quantification method, we were unable to show a difference in inflammatory mediators present in portal blood between DBD and DCD donors. We did however find increased levels of hepatic macrophage activation and evidence of more severe biliary injury in samples collected from DCD donors.

The second part of this thesis focuses on the application of machine perfusion techniques. Machine perfusion could facilitate the use of more marginal grafts and opens up opportunities to assess viability prior to transplantation. In this thesis, we systematically reviewed all machine perfusion protocols currently applied in preclinical or clinical transplantation settings. Hypothermic perfusion (HMP), mid-thermic perfusion (MMP) and normothermic machine perfusion (NMP) have been applied and each centre described the method as safe and feasible. Only small case series have been conducted so far. Nevertheless, oxygenated HMP of DCD livers prior to transplantation lead to a significant reduction in ITBS formation compared to nonperfused controls. In our centre, we developed a normothermic machine perfusion apparatus and perfused ten livers that were declined for transplantation. Using the suitability criteria of lactate clearance within the first two hours of perfusion, we estimated that $7 / 10$ rejected livers could have been suitable for transplantation. When assessing biliary injury we found that degree of injury to the common bile duct did not correlate with the level of injury observed to the large intra-hepatic bile ducts.

In summary, this thesis has identified the gross underutilisation of DCD donor livers in Australia. It furthermore explored the role of endotoxins as a co-factor in the development of biliary injury, which is currently considered the Achilles heel of DCD liver transplantation. The application of machine perfusion was established and hepatic viability could be assessed under normothermic conditions. Future work focussing on the assessment of biliary viability is needed to facilitate the safe use of DCD donor livers. 


\begin{abstract}
Dutch)
Levertransplantatie is vaak het laatste redmiddel voor patiënten met leverfalen. Helaas zijn er niet genoeg donorlevers beschikbaar voor alle patiënten op de wachtlijst, waardoor er wordt overwogen om marginale levers te gebruiken voor transplantatie. Deze levers, afkomstig van oudere danwel non-heart-beating donoren of met hooggradige steatose, zijn gevoeliger voor reperfusie schade. Dit heeft tot gevolg dat deze levers soms niet werken na transplantatie. Daarnaast vormen galwegcomplicaties zoals galwegstricturen voor veel morbiditeit, wat resulteert in meer re-interventies, re-transplantaties en inferieure leverfunctie op de langere termijn. In dit proefschrift wordt een serie studies beschreven die zich focust op mogelijke factoren die een rol spelen in the pathofysiologie van deze galwegstricturen. Daarnaast wordt het gebruik van een leverperfusietechniek beschreven, welke wordt ingezet om de fysiologie van de humane galwegen te bestuderen.
\end{abstract}

Een review van alle Australische orgaandonoren tussen 2005 en 2014, liet zien dat er tijdens deze jaren een stijging van $17 \%$ was in het aantal levers dat niet werd geaccepteerd voor transplantatie. Non-heart-beating donoren waren de voornaamste oorzaak van deze stijging. Maar 62 (13\%) levers afkomstig van non-heart-beating donoren werden gebruikt voor transplantatie gedurende de studieperiode. Tevens waren hogere leeftijd, obesitas en diabetes geassocieerd met het niet accepteren van levers voor transplantatie.

De pathofysiologie van galwegstricturen na levertransplantatie is grotendeels onbekend. Een studie, beschreven in dit proefschrift, heeft geconstateerd dat toediening van lipopolysacchariden (LPS) kan leiden tot ernstige schade aan de kleine galwegen. Cholangiocyten vertoonden degeneratieve veranderingen, infiltratie van inflammatoire cellen en proliferatie, zes uur na de intra-peritoneale toediening van LPS. Deze veranderingen waren verder geassocieerd met een toegenomen permeabiliteit van de blood-gal-barrière en klinische tekenen van cholestase.

LPS kan leiden tot Kupffercel activatie en dit zou een rol hebben kunnen gespeeld in de ontwikkeling van deze LPS-gemedieerde galwegschade. Om dit verder te bestuderen, werd een extra groep ratten voorbehandeld met liposomen die gevuld waren met clodronaat om zodoende Kupffercellen te verwijderen uit hun lever. Deze ratten vertoonden een gelijke hoeveelheid galwegschade na toediening van LPS, wat dus suggereert dat Kupffercellen geen rol spelen in de LPS gemedieerde galwegschade. 
De bevindingen in de rattenstudies leidden tot de initiatie van een studie met humane orgaandonoren. In dit cohort werden de concentratie van endotoxines en andere inflammatoire factoren in portoveneus bloed van heart-beating en non-heart-beating donoren met elkaar vergeleken. De hypothese was dat portoveneus bloed van nonheart-beating donoren een hogere concentratie pro-inflammatoire factoren zou bevatten, als gevolg van hypoxie en hypoperfusie van de darm gedurende orgaandonatie. Zowel de Lumulus Amebocyte Lysate assay als een in vitro assay werden gebuikt, echter werd er geen verschil gevonden in de hoeveelheid inflammatoire mediatoren tussen deze twee typen orgaandonoren. Desondanks, werd er een verhoogde activatie van macrophagen in de lever vastgesteld; en galwegbiopten van non-heart-beating donoren vertoonden meer galwegschade in vergelijking met heart-beating donoren.

Het tweede gedeelte van dit proefschrift richt zich op het gebruik van marginale donorlevers middels toepassing van machineperfusie (MP). Een van de belangrijkste voordelen van MP is dat het de mogelijkheid biedt om levers die geschikt zijn voor transplantatie the differentiëren van levers die uiteindelijk onvoldoende functioneren. In dit proefschrift wordt allereerst een systematic review beschreven omtrent het gebruik van MP-protocollen in pre-klinisch en klinisch onderzoek. Hypotherme perfusie, mid-therme perfusie en normotherme perfusie zijn elk gebruikt voor de preservatie van humane levers en deze technieken worden gezien als veilig en bruikbaar. Tevens ontwikkelden patiënten die een hypotherm geperfuseerde nonheart-beating donorlever ontvingen significant minder galwegstricturen in vergelijking met patiënten die een non-heart-beating donorlever ontvingen die niet werd geperfuseerd.

Er werd een normotherm MP-protocol ontwikkeld in het Princess Alexandra Ziekenhuis in Brisbane (Australië) en tien levers die als ongeschikt waren bestempeld voor transplantatie werden hierop geperfuseerd. Op basis van de lactaatklaring gedurende de perfusie, zouden zeven levers van deze groep mogelijk bruikbaar zijn geweest voor transplantatie. Schade aan de ductus choledochus was echter onvermijdelijk, ongeacht de metabole functie van de lever. De hoeveelheid schade aan de ductus correleerde echter niet met de hoeveelheid schade aan de intrahepatische grote galwegen.

Samengevat, hebben de studies geïncludeerd in dit proefschrift aangetoond dat levers afkomstig van non-heart-beating orgaandonoren nu nauwelijks worden gebruikt voor transplantatie in Australië. Verder werden endotoxines geïdentificeerd als een mogelijk bijdragende factor aan de ontwikkeling van galwegschade, iets dat wordt gezien als de belangrijkste limiterende factor in het gebruik van non-heart-beating donorlevers voor transplantatie. Daarnaast werd een normotherm MP-protocol 
ontwikkeld, dat kan worden gebruikt om de leverfunctie en bruikbaarheid voor transplantatie te testen. Toekomstige studies dienen zich te focussen op het ontwikkelen van een methode om galwegschade beter in kaart te brengen, om zo veilig non-heart-beating donorlevers te kunnen gebruiken voor transplantatie. 


\section{Declaration by author}

This thesis is composed of my original work, and contains no material previously published or written by another person except where due reference has been made in the text. I have clearly stated the contribution by others to jointlyauthored works that I have included in my thesis.

I have clearly stated the contribution of others to my thesis as a whole, including statistical assistance, survey design, data analysis, significant technical procedures, professional editorial advice, and any other original research work used or reported in my thesis. The content of my thesis is the result of work I have carried out since the commencement of my research higher degree candidature and does not include a substantial part of work that has been submitted to qualify for the award of any other degree or diploma in any university or other tertiary institution. I have clearly stated which parts of my thesis, if any, have been submitted to qualify for another award.

I acknowledge that an electronic copy of my thesis must be lodged with the University Library and, subject to the policy and procedures of The University of Queensland, the thesis be made available for research and study in accordance with the Copyright Act 1968 unless a period of embargo has been approved by the Dean of the Graduate School.

I acknowledge that copyright of all material contained in my thesis resides with the copyright holder(s) of that material. Where appropriate I have obtained copyright permission from the copyright holder to reproduce material in this thesis. 
Publications included in this thesis

Chapter 3: J Reiling, KR Bridle, M Gijbels, FG Schaap, L Jaskowski, N Santrampurwala, U Britton, CM Campbell, SWM Olde Damink, DHG Crawford, CHC Dejong, J Fawcett, Low-dose lipopolysaccharide causes biliary injury by blood biliary barrier impairment in a rat hepatic ischemia/reperfusion model. Liver Transplantation, 2016, accepted for publication

\begin{tabular}{ll}
\hline Contributor & Statement of contribution \\
\hline Janske Reiling & $\begin{array}{l}\text { Designed }(70 \%) \text { and conducted }(80 \%) \text { the } \\
\text { experiments, performed data analysis (100\%), } \\
\text { wrote the first version of the paper (65\%) }\end{array}$
\end{tabular}

KR Bridle

Helped design the experiment (10\%), conducted the study (10\%), edited and approved the final version of the paper (5\%)

M Gijbels, CM Campbell

L Jaskowski

LJ Britton, N Santrampurwala, FG Schaap, SWM Olde Damink

DHG Crawford, CHC Dejong, J Fawcett Designed experiments (10\%), oversight of experiment, edited and approved the final version of the paper (10\%)

Chapter 7: J Reiling, DSR Lockwood, AH Simpson, CM Campbell, KR Bridle, N Santrampurwala, L Britton, DHG Crawford, CHC Dejong, J Fawcett, Urea production during Normothermic Machine Perfusion- Price of Success? Liver Transplantation, 2015 May; 21(5):700-3 


\begin{tabular}{|c|c|}
\hline Contributor & Statement of contribution \\
\hline J Reiling & $\begin{array}{l}\text { Designed }(70 \%) \text { and conducted }(80 \%) \text { the } \\
\text { experiments, performed data analysis }(100 \%) \text {, } \\
\text { wrote the first version of the paper }(70 \%)\end{array}$ \\
\hline DSR Lockwood, AH Simpson & $\begin{array}{l}\text { Helped design the experiment (10\%), } \\
\text { conducted the study }(20 \%) \text {, edited and } \\
\text { approved the final version of the paper }(5 \%)\end{array}$ \\
\hline CM Campbell & $\begin{array}{l}\text { Performed the histological assessment of the } \\
\text { liver and bile duct section ( } 100 \%) \text {, edited and } \\
\text { approved the final version of the paper ( } 5 \%)\end{array}$ \\
\hline $\begin{array}{l}\text { KR Bridle, LJ Britton, } \\
\text { N Santrampurwala }\end{array}$ & $\begin{array}{l}\text { Helped design the experiment }(10 \%) \text {, edited } \\
\text { and approved the final version of the paper } \\
(10 \%)\end{array}$ \\
\hline DHG Crawford, CHC Dejong, J Fawcett & $\begin{array}{l}\text { Designed experiments }(10 \%) \text {, oversight of } \\
\text { experiment, edited and approved the final } \\
\text { version of the paper (10\%) }\end{array}$ \\
\hline
\end{tabular}


Contributions by others to the thesis

\section{Concept and design of the project}

Dr Kim Bridle, Professor Darrell Crawford, Professor Kees Dejong and Professor Jonathan Fawcett contributed to the experimental design of each project.

\section{Tissue processing and sectioning}

Formalin fixed liver and bile duct sections from the rat experiments were processed and sectioned by the histotechnology facility at the QIMR Berghofer Medical Research Institute. They furthermore performed the Haematoxylin and eosin staining of these sections. The human liver and common bile duct sections were processed and stained by Envoi Pathology.

\section{Histology scoring}

Liver and bile duct histology scoring (Chapter 3,4 and 8) was performed by Catherine Campbell (Envoi Pathology) and Marion Gijbels (Department of Pathology and Department of Molecular Genetics, Cardiovascular Research Institute Maastricht, the Netherlands), both specialist pathologists.

\section{Serum analysis during normothermic machine perfusion}

Hourly serum samples were collected during normothermic machine perfusion of discarded human donor livers (Chapter 7 and 8) and sent to Queensland Pathology for analysis.

\section{Design of the perfusion circuit used for normothermic machine perfusion}

The perfusion circuit used for the perfusions of the discarded human donor livers (Chapter 7 and 8) was co-designed by Dr Andrew Simpson and manufactured by Cellplex Pty, Ltd, Melbourne, Australia.

\section{Liquid chromatography of bile samples}

Bile samples (Chapter 3 ) were sent to the department of Laboratory Medicine at the University Medical Centre Groningen, The Netherlands, for assessment of bile salt composition using UHPLC-MS/MS.

\section{Cytokine array serum and bile samples}

The concentrations of several cytokines in bile and serum samples included in Chapter 3 and 4 were measured using multiplex ELISA arrays. The arrays was performed in our lab and sent to RayBiotech (Norcross, MA, USA) for assessment of fluorescence intensity. The obtained results were analysed in our lab using specific software. 


\section{Data collection of organ donors}

Organ donor data was obtained from The Australian and New Zealand Organ registry (ANZOD), and used for Chapter 2. When data was not available in the registry, donor records held by DonateLife Queensland were reviewed with the help of Jade Carey, donation specialist.

\section{Assistance with experiments}

Dr Kim Bridle has assisted with the animal experiments (Chapter 3 and 4) and Lesley Jaskowski has performed several Immunohistochemical and Immunofluorescence stains, as well as qRT-PCR's.

NF-KB-dependent cell-based assay and Limulus Amebocyte Lysate quantification method

Professor Matt Brown, and other members of his laboratory (Centre for Inflammation and Disease Research, Institute for Molecular Bioscience, University of Queensland), kindly assisted with the analysis of portal blood samples using a NF-kB-dependent cellbased assay (Chapter 5). Dr Ashok Raj furthermore assisted with the measurement of endotoxins in portal blood samples using the Limulus Amebocyte Lysate quantification method (Chapter 5).

\section{Critical review of the thesis}

Dr Kim Bridle, Professor Darrell Crawford, Professor Kees Dejong and Professor Jonathan Fawcett reviewed and edited each chapter of this thesis and approved the final version. 
Statement of parts of the thesis submitted to qualify for the award of another degree

None 
